\title{
Topological Invariants for Non-Archimedean Bornological Algebras
}

\author{
Dissertation \\ for the award of the degrees \\ "Doctor rerum naturalium" and "Doctor of Philosophy" \\ of the Georg-August-Universität Göttingen and the University of \\ Copenhagen \\ within the doctoral programs of the \\ "PhD School of Mathematical Sciences (SMS)" and the "PhD School \\ of Science" \\ of the Georg-August University School of Science (GAUSS) and the \\ University of Copenhagen \\ submitted by \\ Devarshi Mukherjee \\ from Calcutta, India
}

Göttingen, 2020 

Thesis advisory committee:

Prof. Dr. Ralf Meyer

Mathematisches Institut, Universität Göttingen

Professor Ryszard Nest

Mathematical Institute, University of Copenhagen

\section{Members of the examination committee:}

\section{Referees:}

Prof. Dr. Thomas Schick

Mathematisches Institut, Universität Göttingen

Professor Yakov Kremnitzer

Mathematical Institute, University of Oxford

Dr. Dustin Clausen

Department of Mathematical Sciences, University of Copenhagen

\section{Other members of the defense committee:}

Prof. Dr. Damaris Schindler

Mathematisches Institut, Universität Göttingen

Prof. Dr. Gerlind Plonka-Hoch

Institut für Numerische und Angewandte Mathematik, Universität Göttingen

Dr. Christian Voigt

School of Mathematics and Statistics, University of Glasgow

Date of oral examination: 24.09 .2020 


\section{Contents}

\begin{tabular}{ll}
\hline Abstract & 5
\end{tabular}

\begin{tabular}{ll}
\hline Abstrakt & 7
\end{tabular}

\begin{tabular}{lr}
\hline Acknowledgments & 9 \\
\hline
\end{tabular}

Introduction 11

\begin{tabular}{ll}
\hline Background for the work in this thesis & 12
\end{tabular}

Summary of papers in this thesis 13

$\begin{array}{lll}\text { Chapter 1. Dagger completions and bornological torsion-freeness } & 21\end{array}$

1. Introduction 21

2. Basic notions 22

$\begin{array}{lll}3 . & \text { Spectral radius and semi-dagger algebras } & 28\end{array}$

4. Bornological torsion-freeness 30

$\begin{array}{lll}5 . & \text { Dagger algebras } & 35\end{array}$

6. Dagger completions of monoid algebras 35

\begin{tabular}{lll}
\hline 7. & Dagger completions of crossed products & 37
\end{tabular}

$\begin{array}{lll}\text { Chapter 2. } & \text { Non-archimedean analytic cyclic homology } & 43\end{array}$

1. Introduction 43

$\begin{array}{lrr}2 . & \text { Preparations } & 46\end{array}$

3. Definition of analytic cyclic homology 56

4. Analytic nilpotence and analytically quasi-free resolutions 59

5. Excision 75

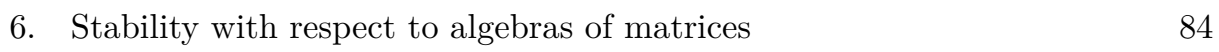

7. Morita functoriality 85

\begin{tabular}{lll}
\hline 8. & Leavitt path algebras & 87
\end{tabular}

\begin{tabular}{lll}
\hline 9. & Filtered Noetherian rings and analytic quasi-freeness & 91
\end{tabular}

$\begin{array}{lll}\text { Chapter 3. A cyclic homology theory in positive characteristic } & 99\end{array}$

1. Introduction 99

$\begin{array}{lll}2 . & \text { Analytic cyclic theory in mixed characteristic } & 101\end{array}$

3. The exact category of locally split extensions 107

\begin{tabular}{lll}
\hline 4. & Independence of the choice of lifting & 117
\end{tabular}

5. Homotopy invariance and stability 121

\begin{tabular}{lll}
\hline 6. & Nilpotent extensions and excision & 122
\end{tabular}

$\begin{array}{lll}\text { Chapter 4. Conclusion and outlook } & 133\end{array}$

1. What is still missing? 133

$\begin{array}{ll}2 . & \text { Some future directions } \\ \end{array}$ 
Bibliography 


\begin{abstract}
In this thesis, we define a cyclic homology theory for non-archimedean bornological algebras, which we call analytic cyclic homology. Let $V$ be a complete, discrete valuation ring with uniformiser $\pi$, residue field $\mathbb{F}=V / \pi V$, and quotient field $F$. The material we present is divided in three parts. In the first part, we interpret Monsky and Washnitzer's weak completion using the framework of bornologies, developed in 11. Weakly complete algebras are used to define Monsky-Washnitzer cohomology, and have three characteristic features - bornological torsion-freeness, completeness and a certain spectral radius condition. We study the homological inheritence of each of these three conditions, and call a $V$-algebra with these properties a dagger algebra.

In the second part, we define analytic cyclic homology for projective systems of complete, bornologically torsion-free $V$-algebras. The theory we develop satisfies homotopy invariance, Morita invariance and excision. We use these properties to compute our theory for (dagger completed) Leavitt path algebras, and tensor product with such algebras. We show that our theory coincides with Berthelot's rigid cohomology for smooth commutative $V$-algebras of relative dimension 1.

In the third part, we define analytic cyclic homology for algebras over the residue field $\mathbb{F}$, by lifting them to free algebras over $V$ and then building dagger completed tube algebras. We show that under very mild assumptions on the bornology of an $\mathbb{F}$-algebra $A$, any complete, bornologically torsion-free $V$-module lifting can be used to compute its analytic cyclic homology. The theory we define satisfies polynomial homotopy invariance, matricial stability, and excision for extensions of finitely generated $\mathbb{F}$-algebras.
\end{abstract}





\begin{abstract}
Abstrakt
Hovedresultat af denne arbejde er en konstruktion af Analytisk homologi, en version af cyklisk homologi for ikke-arkimidiske bornologiske algebra. Lad $V$ være en fuldstændig diskrete valueringsring, $\mathbb{F}=V / \pi V$ dens restklasselegeme og $F$ dens kvotientlegeme. I den første del ve interpreterer Monsky og Washnitzer svag fuldstændigørelse i sprog af bornologiske algebra. Svagt fuldstændige algebra er anvendt til at definere Monsky-Washnitzer kohomologi og har tre karakteristiske eenskaber. De er torsionsfrie (som bornologiske algebra), fuldstændige og har deres spektrum har et bestemt spektral egenskab, indført i teksten nedenunder. Algebra som opfylder de egenskaber kaldes dagger algebra Den første del af tesen er brugt til at analysere homologisk stabilitet af dagger egenskaben.

Den anden del af arbejde introducerer analytisk cyklisk homologi af dagger algebra. Den tilsvarende konstruktion er homotopi invariant, opfylder Morita invariance og har udskærings egenskab. vi regner ud analytisk cyklsk homologi for tensor produkter med Lewitt sti-algebra. En af hovedresultater i denne del er bevis for, at for glatte $V$-algebra, analytisk cyklisk homologi stemmer overens med Bertholet's rigid kohomologi.

Den sidste del af arbejde giver en konstruktion af analytisk cyklisk homologi for algebraer(over $\mathbb{F}$ ved at bruge et løft til en algebra over $V$. Vi viser at, under meget milde betingelser for algebra over $\mathbb{F}$, konstruktion er uafhængig af valg af løftet. Den teori som opnås herved opfylder polynomial homotopi invariance, Morita invariance og udskærings aksiomet for udvidelser af endelig genererede $\mathbb{F}$-algebra.
\end{abstract}





\section{Acknowledgments}

First of all, I would like to thank my principal co-supervisor Ralf Meyer for his immense support and patience, without which the work of this thesis would not have been conceivable. I have over the years learned vast amounts of Mathematics from him, spanning many different areas of the subject. His generosity in sharing his thoughts is something I will always be grateful for.

Secondly, I would like to thank my co-supervisor Ryszard Nest for not only providing me with valuable input for my project, but also broadening my horizons

to other areas of Mathematics. The discussions with him often gave me a completely different perspective on the problems I had been working on as part of my thesis. Finally, I would also like to thank him for the hospitality during my visits to Copenhagen, which I always enjoyed.

I would also like to thank my collaborator Guillermo Cortiñas for letting me into the project of which my thesis is part. This project was originally conceived of by him and Joachim Cuntz, and their continuous input and feedback have been extremely crucial. I am also grateful for all the support I received from him surrounding my overall career prospects.

Finally, I would also like to thank my girlfriend Simran, my family in Hyderabad and my friends from Delhi and Göttingen for all the emotional support, without which these three years would have been unimaginably hard. 



\section{Introduction}

The use of invariants in the understanding of geometry has a long and illustrious history. Such invariants have been applied in areas ranging from the classification of Platonic solids in Greek geometry, to the formulation of the Weil conjectures using certain cohomology theories, to the theory of topological insulators using $K$-theory. We dedicate this introductory section to an informal discussion on invariants for two types of 'geometries' - geometry in characteristic zero, and geometry in positive characteristic.

By geometry in characteristic zero, we mean associative algebras over fields of characteristic zero, that encode some geometric structure 1 For example, affine algebraic varieties over an algebraically closed field are equivalent to finitely generated commutative algebras over the field. Another example is the Gelfand-Naimark Theorem, which states that commutative $\mathrm{C}^{*}$-algebras are the same as locally compact Hausdorff spaces. Regarding varieties or schemes over the complex numbers as topological spaces, we can speak of their singular cohomology groups, which is a powerful invariant originating from algebraic topology. If we consider spaces that are suitably nonsingular, then a related invariant is the de Rham cohomology of say, a smooth manifold or a non-singular variety over $\mathbb{C}$. These invariants can be used to obtain important geometric information such as the Euler characteristic of a topological space $X$ or the Lefschetz number of a continuous map on $X$. When we replace 'spaces' in the classical sense of topology or algebraic geometry by possibly non-commutative algebras, singular cohomology and de Rham cohomology are no longer meaningful, and need to be replaced with cyclic homology. The study of non-commutative algebras as an extension of 'classical' geometry is the subject matter of Alain Connes' non-commutative geometry.

As the reader can probably guess, geometry in positive characteristic refers to associative algebras over fields of characteristic $p>0$. Algebraic geometry in this context has a rich history, stemming from the pursuit reasonable invariants to prove the Weil conjectures. From the purview of non-commutative geometry, interesting geometric examples arise from finite group actions on affine schemes over finite fields, which are treated by crossed product algebras. Another class of examples is graph algebras over finite fields. It turns out, however, that the positive characteristic of the underlying ring or field poses problems that invariants such as de Rham cohomology or cyclic homology cannot easily circumvent. For instance, the Poincaré Lemma says that in characteristic zero, the de Rham complex of the (contractible) affine $n$-space is exact. The proof uses that the integration map on differential forms yields sections for the de Rham differential. This is however no longer true in positive characteristic.

\footnotetext{
${ }^{1}$ I should mention here that throughout this thesis, I will only talk about geometric objects that are in some sense affine. That is, I will never refer to projective schemes, or graded algebras.
} 
What seems desirable is a de Rham or cyclic type cohomology theory that is defined on a suitable category of 'characteristic 0 liftings' of an algebra over characteristic $p>0$. Of course, the resulting lifting and invariants should be defined in a way that we can still obtain information about the original geometric object. Considerations of this nature led to the development of several related p-adic cohomology theories. Two such invariants which motivate this thesis are Monsky-Washnitzer cohomology and rigid cohomology. The goal of this thesis to study liftings of geometric objects in positive characteristic, to objects that are better behaved in characteristic zero. Finally, we use such liftings to construct a well-behaved homological invariant that specialises to Monsky-Washnitzer and rigid cohomology for certain classes of commutative algebras.

\section{Background for the work in this thesis}

For smooth, commutative algebras over rings containing the rationals $\mathbb{Q}$, the Hochschild-Kostant-Rosenberg Theorem implies that de Rham cohomology coincides with periodic cyclic homology. In other words, periodic cyclic homology extends de Rham cohomology to non-commutative algebras in characteristic zero. This thesis takes a step in the direction of extending rigid cohomology to non-commutative algebras over finite fields.

The starting point of this program is the identification of periodic cyclic homology with rigid cohomology for commutative, finite type algebras (see $[\mathbf{1 1}]$ ). We describe this identification here. Denote by $V$ a complete discrete valuation ring with uniformiser $\pi, \mathbb{F}=V / \pi V$ its residue field, and $F$ its fraction field, which we assume to be of characteristic zero - we fix this notation for the rest of this thesis. Let $A$ be a commutative $\mathbb{F}$-algebra, and $I \nrightarrow V[S] \rightarrow A$ be a free commutative algebra presentation of $A$. Here $S$ is a generating set of $A$, and for a natural choice, we can choose $S=A$. The next step is to construct certain bornological versions of $I$-adic completions $\underline{R}_{I, 1 / n}$ of $R=V[S]$, with $\underline{R}:=R \otimes F$ and $n \in \mathbb{N}$. Concretely, the bornology is defined by specifying bounded subsets $M \subseteq R$ for which $\rho(M)<\epsilon^{1 / n}$, where $\epsilon=|\pi|$ is the norm of the uniformiser of $V$. This provides a bornological interpretation of the weak completions used in Monsky-Washnitzer cohomology [32. Section 2]. In conclusion, we get a projective system of complete, bornological $F$-algebras $\left(\underline{R}_{J, 1 / n}\right)_{n \in \mathbb{N}}$.

Definition. Let $A$ and $R$ be as above. The periodic cyclic homology of $A$ is defined as the homology of the homotopy inverse limit of the projective system of periodic cyclic complexes

$$
\left(\mathbb{H} \mathbb{P}\left(\underline{R}_{J, 1 / n}\right), B+b\right)_{n \in \mathbb{N}} .
$$

The main result of this paper shows that the homotopy inverse limit of $\left(\mathbb{H} \mathbb{P}\left(\underline{R}_{J, 1 / n}\right), B+b\right)_{n \in \mathbb{N}}$ is quasi-isomorphic to the homotopy inverse limit of the de

Rham complexes $\left(\underline{R}_{J, 1 / n} \otimes_{\underline{R}} \Omega_{R}^{*}, d\right)$, made 2-periodic. The results of Große-Klönne 19 imply that this homotopy inverse limit of de Rham complexes computes the rigid cohomology of $A$. In summary, we have the following:

Theorem. 11. Theorem 6.5] Let $A$ be a finitely generated commutative $\mathbb{F}$ algebra. Then for $*=0,1$, we have

$$
\mathrm{HP}_{*}(A) \cong \bigoplus_{j \in \mathbb{Z}} H_{\text {rig }}^{2 j-*}(A, F) .
$$


The machinery above has the important feature that it computes rigid cohomology using a chain complex that is defined naturally using free commutative algebra liftings. Furthermore, the homotopy invariance of periodic cyclic homology can be used to show that if we use a different generating set $S \subset A$ for the free commutative algebra lifting $R=V[S]$, we still get the same periodic cyclic homology. However, it is unclear how this definition can be extended to non-commutative algebras in a manner that is independent of the choice of the lifting. Invariance under different liftings is a key result about Monsky-Washnitzer cohomology, proven by Marius van der Put (see [12 Remark 8.2.8]). This motivates the need for a variant of periodic cyclic homology that is defined for non-commutative $\mathbb{F}$-algebras, and is independent of choices of intermediate liftings to $V$-algebras. Such a homology theory should of course have all the desirable formal properties that periodic cyclic homology possesses. Finally, this theory should specialise in the smooth, commutative case to periodic cyclic homology, which coincides with rigid cohomology. While we have not been able to prove the last claim in full generality, we have constructed a theory which nevertheless yields interesting computations for several classes of commutative as well as non-commutative algebras.

\section{Summary of papers in this thesis}

This thesis has been arranged in the form of a collection of three papers I have (co)-authored during my time as a $\mathrm{PhD}$ student.

Dagger completions and bornological torsion-freeness (joint with Ralf Meyer). ${ }^{2}$

Here we describe the dagger completion of a bornological $V$-algebra, which is a key ingredient in the construction of our homology theory. It was already observed in the seminar works of Monsky and Washnitzer, that in order to obtain a well-behaved de Rham cohomology theory, we need to 'complete' a torsion-free $V$-algebra lifting $R$ of an $\mathbb{F}$-algebra. They also observed that one cannot simply work with $\pi$-adic completions and ensure the finite dimensionality of de Rham cohomology, even in simple cases such as the affine line $\mathbb{F}$. So the right thing to do is to take a certain subalgebra $R^{\dagger}$ of the $\pi$-completion $\widehat{R}$, which they call the weak completion. If $R$ is a polynomial algebra, then $R^{\dagger}$ consists of overconvergent power series. Quotients of dagger completed polynomial algebras appear naturally in the context of Große-Klönne's theory of rigid analytic spaces with overconvergent structure sheaf.

In this paper, we describe the dagger completion process and its various inheritence properties from the perspective of homological algebra. Our treatment uses bornologies, which is the framework we choose for our cyclic homology theories. We briefly recall some definitions from bornological analysis to keep the summaries of the papers readable:

\section{DeFinition.}

- A bornology on a set $S$ is a collection of its subsets, called bounded sets, such that all finite subsets are bounded, and subsets and finite unions of bounded subsets are bounded. A bounded function $f: S \rightarrow T$ between bornological sets is one which maps bounded sets to bounded sets.

\footnotetext{
${ }^{2}$ This paper is published in the Quarterly Journal of Mathematics (see 27 .).
} 
- A bornological $V$-module is a $V$-module $M$ with a bornology $\mathcal{B}_{M}$, such that any bounded subset is contained in a bounded $V$-submodule. A bornological $V$-algebra $R$ is a bornological $V$-module with a bounded multiplication map $R \times R \rightarrow R$.

ExAmple. We can always equip a $V$-module $M$ with the collection of all finitely generated $V$-submodules. This is called the fine bornology.

The fine bornology is the smallest bornology on a $V$-module. It is in essence the 'base' bornology from the viewpoint of our applications, upon which we perform further analytical operations. This is because the algebras we are interested in such as Leavitt path algebras, and coordinate rings of smooth curves - come with no further analytical structure.

We now turn to the completion of a bornological $V$-module. A bornological $V$-module $M$ is said to be complete is every bounded subset $S \in \mathcal{B}_{M}$ is contained in a bounded $\pi$-adically complete $V$-submodule $T$. To define the completion of $M$ in the most enlightening manner possible, we state a result on the structure of bornological $V$-modules:

Proposition. [11, Proposition 2.5] The category of bornological $V$-modules with bounded $V$-module maps is equivalent to the full subcategory of inductive systems of $V$-modules, with injective structure maps.

Definition. Let $M$ be a bornological $V$-module, written as an inductive limit of $V$-submodules $M=\underline{\lim } M_{i}$. Let $\widehat{M}_{i}$ denote the $\pi$-adic completion of $M_{i}$. Then the completion $\overparen{M}$ of $\vec{M}$ is defined as the quotient of the inductive $\operatorname{limit} \stackrel{\lim }{\longrightarrow} \widehat{M}_{i}$ by the bornological closure of the trivial $V$-module $\{0\}$.

For the definition of bornological closure, we direct the reader to the actual paper. We only remark that taking this quotient ensures that the completion is bornologically separated, that is, every bounded subset is contained in a $\pi$-adically separated bounded $V$-submodule. Equivalently, the structure maps $M_{i} \rightarrow M_{j}$ of the inductive system defining $M$ remain injective when we take their $\pi$-adic completions $\widehat{M}_{i} \rightarrow \widehat{M}_{j}$. The completion $\overparen{M}$ of a bornological $V$-module $M$ is complete and admits a canonical map $M \rightarrow T$ to it. Furthermore, it has the expected universal property: if $f: M \rightarrow N$ is a bounded $V$-module map into a complete bornological $V$-module $N$, then there exists a unique bounded $V$-module map $M \rightarrow N$ factorising $f$.

Example. Any $V$-module with the fine bornology is complete. This is because finitely generated $V$-modules are already $\pi$-adically complete.

We now talk about torsion-freeness, which is an important aspect of our lifting constructions from the world of $\mathbb{F}$-algebras. Recall, a $V$-module is torsion-free if multiplication by $\pi$ is an injective $V$-module map. It is bornologically torsion-free if this map respects bornologies.

ExAmple. A torsion-free $V$-module $M$ with the fine bornology is bornologically torsion-free.

The example above implies that most algebras we are interested in studying, such as the coordinate ring of a smooth affine variety over $V$ with the fine bornology, are automatically also bornologically torsion-free. However, in order to arrive at the weak completion of Monsky-Washnitzer, we will need to enlarge the bornology on a 
given $V$-algebra, and complete with respect to the enlarged bornology. It is therefore natural to ask whether bornological torsion-freeness is preserved by completions. This is indeed the case:

TheOrEm. 27, Theorem 4.6] Let $M$ be a bornologically torsion-free bornological $V$-module. Then $\vec{M}$ is bornologically torsion-free.

Finally, we come to the growth condition of bounded subsets that is inherent in the weak completion of Monsky-Washnitzer. A bornological $V$-algebra $A$ is called semi-dagger if whenever $S$ is a bounded $V$-submodule, we have $\pi \cdot S^{2} \subseteq$ $S$. Trivially, any $\mathbb{F}$-algebra with the fine bornology is semi-dagger. For torsionfree $V$-algebras, however, we need to work with a larger bornology than the fine bornology. Specifically, we equip a torsion-free bornological $V$-algebra $A$ with the bornology generated by $V$-submodules of the form $\sum_{j=0}^{\infty} \pi^{j} S^{j+1}$, where $S$ is a bounded submodule in the original bornology of $A$. This enlarged bornology is called the linear growth bornology. It is by construction the smallest semi-dagger bornology on $A$. We denote a bornological algebra $A$ with the linear growth bornology by $A_{\lg }$. The enlargement of the bornology on $A$ to the linear growth bornology produces a canonical map $A \rightarrow A_{\lg }$ that factorises any bounded algebra homomorphism into a semi-dagger algebra. The following result shows the inheritance properties of linear growth bornology and semi-dagger algebras for the notions previously introduced.

Proposition. 27, Proposition 4.11, Proposition 3.8] If $A$ is a bornologically torsion-free $V$-algebra, then so is $A_{\mathrm{lg}}$. If $A$ is a semi-dagger bornological $V$-algebra, then so is its completion $\vec{A}$.

Combining all the notions we have introduced so far, we can define a dagger algebra:

Definition. A dagger algebra is complete, bornologically torsion-free, semidagger $V$-algebra.

We now show how to construct a dagger algebra from a given bornological $V$-algebra.

Definition. Let $A$ be a bornologically torsion-free $V$-algebra. The dagger completion $A^{\dagger}$ of $A$ is defined as the completion of $A$ in its linear growth bornology, that is, $A^{\dagger}:=\overparen{A_{\lg }}$.

We have only defined the dagger completion of a bornologically torsion-free $V$ algebra since the algebras we are interested in are already bornologically torsion-free. If $A$ is not bornologically torsion-free, we would need to take the image $A_{\mathrm{tf}}$ of $A$ inside $A \otimes F$. The dagger completion of $A$ is then the completion of $A_{\mathrm{tf}}$ in its linear growth bornology. There is a universal map $A \rightarrow A^{\dagger}$ obtained by combining the maps $A \rightarrow A_{\mathrm{tf}}, A \rightarrow A_{\mathrm{lg}}$ and $A \rightarrow \bar{A}$, that factorises a bounded algebra homomorphism into a dagger algebra. And, the various inheritance properties of the three notions that define a dagger algebra imply that the dagger completion $A^{\dagger}$ is indeed a dagger algebra.

An important feature of this bornological formalism is that these definitions make sense also for noncommutative algebras. In Section 6, we compute the dagger completion $V[S]^{\dagger}$ of a monoid algebra $V[S]$, which recovers Monsky-Washnitzer's weak completion if we set $S=\mathbb{N}^{k}$. In Section 7 , we compute the dagger completion 
of the crossed product algebra of a certain kinds of monoid actions. Finally, our main result that is crucial for subsequent developments of the theory is the following inheritence property for extensions of bornological algebras:

Theorem. 27, Theorem 5.2] Let $A \nrightarrow B \rightarrow C$ be an extension of bornological $V$-algebras. If $A$ and $C$ are dagger algebras, so is $B$.

Non-archimedean analytic cyclic homology (joint with Guillermo Cortiñas and Ralf Meyer).

In this article, we define a homology theory for projective systems of complete, bornologically torsion-free bornological algebras (briefly, pro-algebras) over complete discrete valuation rings $V$. The computations so far are directed towards two classes of algebras that are interesting from the perspective of algebraic and noncommutative geometry, namely, coordinate rings of smooth 1-dimensional affine varieties over $V$, and Leavitt path algebras. Furthermore, the formal properties of our theory provide computations for Laurent polynomials in $n$-variables and higher dimensional Toeplitz algebras and Leavitt path algebras, taking us beyond homological dimension 1.

Our construction uses dagger completions of certain generalised tube algebras that are motivated from rigid cohomology. The $m$-th tube algebra of a torsion-free $V$-algebra $A$ with respect to an ideal $I \unlhd A$ is defined as $\mathcal{U}\left(A, I^{m}\right)=\sum_{n=0}^{\infty} \pi^{-\left\lfloor\frac{n}{m}\right\rfloor} I^{m n} \subseteq$ $A \otimes F$. Varying $m$, we get a projective system $\mathcal{U}\left(A, I^{\infty}\right)=\left(\mathcal{U}\left(A, I^{m}\right)\right)_{m}$ of torsionfree bornological algebras, with the subspace bornology inherited from $A \otimes K$. In our context, we use the tube algebra of the tensor algebra presentation $\mathrm{J} R \rightarrow \mathrm{T} R \rightarrow R$, of a pro-algebra $R$. Taking a certain relative version of dagger completion ${ }^{4}$ of the tube algebra $\mathcal{U}\left(\mathrm{T} R, \mathrm{~J} R^{\infty}\right)$ with respect to the canonical ideal $\mathcal{U}\left(\mathrm{J} R, \mathrm{~J} R^{\infty}\right)$, we obtain a pro-algebra $\mathcal{T} R$, which fits in an extension

$$
\mathcal{J} R \nrightarrow \mathcal{T} R \rightarrow R
$$

with an appropriately nilpotent kernel $\mathcal{J} R$.

The definition of our homology theory uses a certain quotient of the periodic cyclic bicomplex $(\mathbb{H} \mathbb{P}, B+b)$, called the $X$-complex. We do not define this here and instead direct the reader to Section 2.7 for its definition.

Definition (Analytic cyclic homology). Let $R$ be a pro-algebra. Its analytic cyclic complex $\mathbb{H} \mathbb{A}(R)$ is defined as the projective system of chain complexes $X(\mathcal{T} R \otimes$ $F)^{5}$. The homology of the homotopy inverse limit of $\mathbb{H} \mathbb{A}$ is called the analytic cyclic homology of $R$.

Our homology theory satisfies the following formal properties:

- Homotopy invariance for dagger homotopies (see Definition 4.1.1);

- Matricial stability and more generally, Morita invariance (Section 6 and Section 7);

- Excision for semi-split extensions of pro-algebras (Section 5).

\footnotetext{
${ }^{3}$ This paper is accepted for publication in Documenta Mathematica.

${ }^{4}$ In light of 27 . Theorem 5.2], we must use this more technical completion in place of the 'absolute' dagger completion of the tube-tensor algebra, because the quotient $\mathcal{U}\left(\mathrm{T} R, \mathrm{~J} R^{\infty}\right) / \mathcal{U}\left(\mathrm{J} R, \mathrm{~J} R^{\infty}\right) \cong$ $R$ is not assumed to be semi-dagger.

${ }^{5}$ You can read the $X$-complex as $\mathbb{H} \mathbb{P}$ for now. In what follows, we shall explain why it makes no difference whether we use the $X$-complex or the periodic cyclic complex of $\mathcal{T} R$
} 
We note here that the reason we tensor the $X$-complex with $F$ in the definition of the analytic cyclic complex is to ensure homotopy invariance, on which several key results rely.

To put our machinery into context, we shed some light on the Cuntz-Quillen approach to cyclic homology theories. Their idea is to build a particular resolution $N \nrightarrow E \rightarrow A$ of an algebra $A$, by an algebra $E$ that has Hochschild cohomological dimension 1, and nilpotent kernel $N$. Nilpotence of the kernel ensures that its periodic cyclic complex is contractible. So the periodic cyclic homology of $E$ is the same as that of $A$. Homological dimension 1 ensures that the periodic cyclic homology of $E$ is computable by the $X$-complex. Finally, homotopy invariance of periodic cyclic homology, and the universal property of 1-dimensional algebras imply that any such extension can be used to compute the periodic cyclic homology of $A$. A significant part of this paper contextualises such results in the non-Archimedean setting. We recall some key definitions that appear in the paper.

Definition (Analytic nilpotence). A pro-algebra $\left(A_{n}, \alpha_{m, n}\right)_{n \in N}$ is nilpotent $\bmod \pi$ if, for each $m \in N$, there are $n \in N_{\geq m}$ and $l \in \mathbb{N}^{*}$ such that $\alpha_{m, n}\left(A_{n}^{l}\right) \subseteq \pi A_{m}$. We call a pro-algebra analytically nilpotent if it is isomorphic to a projective system of dagger algebras and is nilpotent $\bmod \pi$.

In what follows, we shall call extensions of pro-algebras $N \rightarrow E \rightarrow R$ analytically nilpotent if the kernel $N$ is analytically nilpotent. We call an extension semi-split if it splits by a projective system of $V$-module maps. In other words, the splitting map for the cokernel of the extension is only $V$-linear.

Definition (Analytic quasi-freeness). A pro-algebra $R$ is analytically quasi-free if any semi-split, analytically nilpotent extension $N \nrightarrow E \rightarrow R$ splits by a pro-algebra homomorphism $R \rightarrow E$.

One of our main results is the following version of Goodwillie's Theorem:

THEOREM 4.7.1. Let $J \hookrightarrow E \stackrel{p}{\rightarrow} R$ be a semi-split, analytically nilpotent extension of pro-algebras. Then $p$ induces a chain homotopy equivalence $\mathbb{H} \mathbb{A}(E) \simeq$ $\mathbb{H} \mathbb{A}(R)$ and $\mathbb{H} \mathbb{A}(J)$ is contractible. So $\mathrm{HA}_{*}(E) \cong \mathrm{HA}_{*}(R)$ and $\mathrm{HA}_{*}(J)=0$ for $*=0,1$. If $E$ is analytically quasi-free, then $\mathbb{H} \mathbb{A}(R)$ is chain homotopy equivalent to $X(E \otimes F)$ and $\mathrm{HA}_{*}(R)$ is isomorphic to the homology of the homotopy projective limit of $X(E \otimes F)$.

A particularly nice consequence of the theorem above is the case where the algebra $R$ is itself analytically quasi-free.

Corollary 4.7.2. Let $R$ be an analytically quasi-free algebra. Then $\mathbb{H} \mathbb{A}(R)$ is chain homotopy equivalent to $X(R \otimes F)$ and $\mathrm{HA}_{*}(R)$ is isomorphic to the homology of $X(R \otimes F)$.

The algebras mentioned at the beginning of the section, namely Leavitt path algebras and smooth 1-dimensional algebras are analytically quasi-free. So the result above makes our theory computable for them. The following is our main result using analytic quasi-freeness:

THEOREM 9.2.9. Let $X$ be a smooth affine variety over the residue field $\mathbb{F}$ of dimension 1 and let $A=\mathcal{O}(X)$ be its algebra of polynomial functions. Let $R$ be a smooth, commutative algebra of relative dimension 1 with $R / \pi R \cong A$. Equip $R$ with 
the fine bornology and let $R^{\dagger}$ be its dagger completion. If * $=0,1$, then $\mathrm{HA}_{*}\left(R^{\dagger}\right)$ is naturally isomorphic to the de Rham cohomology of $R^{\dagger}$. This is isomorphic to the Monsky-Washnitzer cohomology of $A$, which, if $\operatorname{char}(\mathbb{F})>0$, agrees with the rigid cohomology $H_{\text {rig }}^{*}(A, F)$ of $X$.

We can in principle use analytic quasi-freeness to compute our theory also for Leavitt path algebras, but our coauthor Guillermo Cortiñas pointed out a more elegant way, that uses stability and excision. In what follows, let $E$ be a directed graph with vertices $E^{0}$ and incidence matrix $N_{E}$. Let $C(E)$ and $L(E)$ denote the Cohn and Leavitt path algebras, respectively.

TheOREM 8.1). Let $R$ be a complete bornological algebra. Let $E$ be a graph with countably many vertices. Then

$$
\begin{array}{ll}
\mathbb{H} \mathbb{A}(R \otimes C(E)) \simeq \mathbb{H} \mathbb{A}\left(R \otimes V^{\left(E^{0}\right)}\right), \quad & \mathbb{H} \mathbb{A}(C(E)) \simeq F^{\left(E^{0}\right)}, \\
& \mathbb{H} \mathbb{A}(L(E)) \simeq \operatorname{coker}\left(N_{E}\right) \oplus \operatorname{ker}\left(N_{E}\right)[1],
\end{array}
$$

If $E^{0}$ is finite, then

$$
\begin{aligned}
& \mathbb{H} \mathbb{A}(R \otimes C(E)) \simeq \bigoplus_{v \in E^{0}} \mathbb{H} \mathbb{A}(R), \\
& \mathbb{H} \mathbb{A}(R \otimes L(E)) \simeq\left(\operatorname{coker}\left(N_{E}\right) \oplus \operatorname{ker}\left(N_{E}\right)[1]\right) \otimes \mathbb{H} \mathbb{A}(R) .
\end{aligned}
$$

Corollary 8.2. $\mathbb{H} \mathbb{A}\left(R \otimes V\left[t, t^{-1}\right]\right)$ is chain homotopy equivalent to $\mathbb{H} \mathbb{A}(R) \oplus$ $\mathbb{H} \mathbb{A}(R)[1]$ and $\mathrm{HA}_{*}\left(R \otimes V\left[t, t^{-1}\right]\right) \cong \mathrm{HA}_{*}(R) \oplus \mathrm{HA}_{*}(R)[1]$.

The result above is very powerful as it provides nontrivial computations beyond homological dimension 1. This is because the tensor product of two algebras of homological dimension 1 can in general have homological dimension 2 . We can therefore iterate this result to get computations for tensor products of Laurent polynomial algebras. Finally, all these results remain valid even for dagger completed Cohn and Leavitt path algebras.

Analytic cyclic homology in positive characteristic (joint with Ralf Meyer). 6

In this paper, we define analytic cyclic homology for an $\mathbb{F}$-algebra $A$. The construction proceeds as follows: let $W$ be a complete, bornologically torsion-free $V$-module lifting of $A$, which is viewed as a bornological $V$-algebra with the fine bornology. Here by lifting we mean any bornological quotient map $W \stackrel{\varrho}{\rightarrow} A$. Such a lifting always exists, as we can take the free $V$-module over $A$ with the fine bornology. Thereafter, we follow the Cuntz-Quillen machinery by using the tensor algebra resolution $I \nrightarrow \mathrm{T} W \rightarrow A$ to build tube algebras $\mathcal{U}\left(\mathrm{T} W, I^{\infty}\right)=\left(\mathcal{U}\left(\mathrm{T} W, I^{m}\right)\right)_{m \geq 1}$ of TW with respect to $I$.

A key difference to torsion-free theory developed previously arises here. Since the canonical lifting of $A$ is the free $V$-module $W=V A$, the dagger completions of the tube-tensor algebras $\mathcal{U}\left(T W, I^{m}\right)$ are hard to describe explicitly. So to remedy this, we equip the tube algebras with the linear growth bornology and take their $X$-complex. This yields chain complexes $X\left(\mathcal{U}\left(\mathrm{T} W, I^{\infty}\right)_{\lg }\right):=X\left(\mathcal{U}\left(\mathrm{T} W, I^{m}\right)_{\lg }\right)_{m \geq 1}$ of incomplete bornological $V$-modules.

\footnotetext{
${ }^{6}$ This paper is currently in the form of a manuscript I have written for the purpose of this
} thesis. A journal version will follow shortly. 
Definition (Analytic cyclic homology of $A$ ). The analytic chain complex of $A$ with respect to $W$ is defined as the projective system of chain complexes of bornological $F$-modules $\mathbb{H} \mathbb{A}(A ; W, \varrho):=\left(X\left(\mathcal{U}\left(\mathrm{T} W, I^{\infty}\right)_{\lg } \otimes F\right)\right.$. We call the analytic chain complex of $A$ with respect to the natural lifting $W=V A$ the analytic chain complex $\mathbb{H} \mathbb{A}(A)$ of $A$.

Thereafter, we take the homotopy inverse limit holim $\mathbb{H} \mathbb{A}(A, W, \varrho)$ to obtain a chain complex of bornological $F$-modules. Finally, we complete this chain complex in the following weaker sense:

Definition (Quasi-completion). Let $M$ be a bornological $V$-module, written as an inductive limit $M=\underset{\lim }{\longrightarrow} M_{i}$ of its bornological $V$-modules. The quasi-completion is defined as the inductive limit $\mathcal{Q}(M)=\underline{\lim } \widehat{M_{i}}$.

The homology of the resulting chain complex $\mathcal{Q}(\operatorname{holim}(\mathbb{H} \mathbb{A}(A, W, \varrho)))$ is called the analytic cyclic homology of $A$ with respect to $\varrho: W \rightarrow A$. The primary objective of this paper is to find the correct derived category in which all such liftings yield quasi-isomorphic analytic chain complexes. Such a derived category is constructed using a certain exact category of locally split extensions of torsion-free bornological $V$-modules (Section 3). The statement of the main result is as follows:

Theorem 2.1.8. Let $A$ be an $\mathbb{F}$-algebra, let $W$ be a complete, bornologically torsion-free bornological $V$-module, and let $\varrho: W \rightarrow A$ be a surjective $V$-module map, bounded in the fine bornology on $A$. Then $\varrho$ induces a bounded $V$-algebra

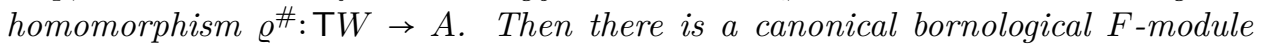
isomorphism

$$
\mathrm{HA}_{*}(A) \stackrel{\simeq}{\rightarrow} \mathrm{HA}_{*}(A ; W, \varrho) .
$$

The theory we define is homotopy invariant for polynomial homotopies and matricially stable (see Section 5). Finally, the second key result of this paper is the following excision theorem:

Theorem (6.3.4). Let $K \rightarrow E \rightarrow Q$ be an extension of finitely generated $\mathbb{F}$ algebras. Then there exists a semi-split extension of pro-dagger algebras $\mathcal{K} \rightarrow \mathcal{E} \rightarrow \mathcal{Q}$ that lifts the original extension of $\mathbb{F}$-algebras. This yields the following long exact sequence in analytic cyclic homology:

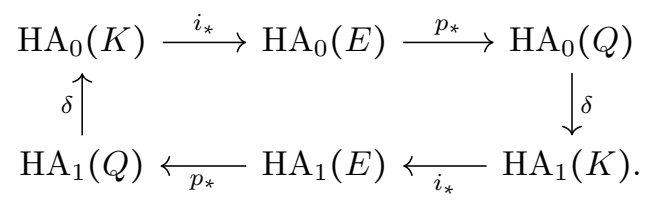





\title{
CHAPTER 1
}

\section{Dagger completions and bornological torsion-freeness}

\begin{abstract}
We define a dagger algebra as a bornological algebra over a discrete valuation ring with three properties that are typical of Monsky-Washnitzer algebras, namely, completeness, bornological torsion-freeness and a certain spectral radius condition. We study inheritance properties of the three properties that define a dagger algebra. We describe dagger completions of bornological algebras in general and compute some noncommutative examples.
\end{abstract}

\section{Introduction}

In [28], Monsky and Washnitzer introduce a cohomology theory for affine nonsingular varieties defined over a field $\mathbb{F}$ of nonzero characteristic. Let $V$ be a discrete valuation ring such that the fraction field $F$ of $V$ has characteristic 0 . Let $\pi \in V$ be a uniformiser and let $\mathbb{F}=V / \pi V$ be the residue field. Monsky and Washnitzer lift the coordinate ring of a smooth affine variety $X$ over $\mathbb{F}$ to a smooth commutative algebra $A$ over $V$. The dagger completion $A^{\dagger}$ of $A$ is a certain subalgebra of the $\pi$-adic completion of $A$. If $A$ is the polynomial algebra over $V$, then $A^{\dagger}$ is the ring of overconvergent power series. The Monsky-Washnitzer cohomology is defined as the de Rham cohomology of the algebra $F \otimes_{V} A^{\dagger}$.

The dagger completion is interpreted in $\mathbf{1 1}$ in the setting of bornological algebras, based on considerations about the joint spectral radius of bounded subsets. The main achievement in $\mathbf{1 1}$ is the construction of a chain complex that computes the rigid cohomology of the original variety $X$ and that is strictly functorial. In addition, this chain complex is related to periodic cyclic homology. Here we continue the study of dagger completions. We define dagger algebras by adding a bornological torsion-freeness condition to the completeness and spectral radius conditions already present in 11. We also show that the category of dagger algebras is closed under extensions, subalgebras, and certain quotients, by showing that all three properties that define them are hereditary for these constructions.

The results in this article should help to reach the following important goal: define an analytic cyclic cohomology theory for algebras over $\mathbb{F}$ that specialises to Monsky-Washnitzer or rigid cohomology for the coordinate rings of smooth affine varieties over $\mathbb{F}$. A general machinery for defining such cyclic cohomology theories is developed in 26. It is based on a class of nilpotent algebras, which must be closed under extensions. This is why we are particularly interested in properties hereditary for extensions.

If $S$ is a bounded subset of a $F$-algebra $A$, then its spectral radius $\varrho(S) \in[0, \infty]$ is defined in 11. If $A$ is a bornological $V$-algebra, then only the inequalities $\varrho(S) \leq s$ for $s>1$ make sense. This suffices, however, to characterise the linear growth bornology on a bornological $V$-algebra: it is the smallest $V$-algebra bornology 
with $\varrho(S) \leq 1$ for all its bounded subsets $S$. We call a bornological algebra $A$ with this property semi-dagger because this is the main feature of dagger algebras. Any bornological algebra $A$ carries a smallest bornology with linear growth. This defines a semi-dagger algebra $A_{\mathrm{lg}}$. If $A$ is a torsion-free, finitely generated, commutative $V$-algebra with the fine bornology, then the bornological completion $\overline{A_{\lg }}$ of $A_{\lg }$ is the Monsky-Washnitzer completion of $A$.

Any algebra over $\mathbb{F}$ is also an algebra over $V$. Equipped with the fine bornology, it is complete and semi-dagger. We prefer, however, not to call such algebras "dagger algebras." The feature of Monsky-Washnitzer algebras that they lack is torsion-freeness. The purely algebraic notion of torsion-freeness does not work well for bornological algebras. In particular, it is unclear whether it is preserved by completions. We call a bornological $V$-module $A$ bornologically torsion-free if multiplication by $\pi$ is a bornological isomorphism onto its image. This notion has very good formal properties: it is preserved by bornological completions and linear growth bornologies and hereditary for subalgebras and extensions. So $\overparen{A_{\mathrm{lg}}}$ remains bornologically torsion-free if $A$ is bornologically torsion-free. The bornological version of torsion-freeness coincides with the usual one for bornological $V$-modules with the fine bornology. Thus $\overline{A_{\mathrm{lg}}}$ is bornologically torsion-free if $A$ is a torsion-free $V$-algebra with the fine bornology.

A bornological $V$-module $M$ is bornologically torsion-free if and only if the canonical map $M \rightarrow F \otimes_{V} M$ is a bornological embedding. This property is very important. On the one hand, we must keep working with modules over $V$ in order to keep the original algebra over $\mathbb{F}$ in sight and because the linear growth bornology only makes sense for algebras over $V$. On the other hand, we often need to pass to the $F$-vector space $F \otimes_{V} M$ - this is how de Rham cohomology is defined. Bornological vector spaces over $F$ have been used recently to do analytic geometry in $[\mathbf{5} \mathbf{7}$. The spectral radius of a bounded subset of a bornological $V$-algebra $A$ is defined in [11 by working in $F \otimes_{V} A$, which only works well if $A$ is bornologically torsion-free. Here we define a truncated spectral radius in $[1, \infty]$ without reference to $F \otimes_{V} A$, in order to define semi-dagger algebras independently of torsion issues.

We prove that the properties of being complete, semi-dagger, or bornologically torsion-free are hereditary for extensions. Hence an extension of dagger algebras is again a dagger algebra.

To illustrate our theory, we describe the dagger completions of monoid algebras and crossed products. Dagger completions of monoid algebras are straightforward generalisations of Monsky-Washnitzer completions of polynomial algebras.

We thank the anonymous referee for helpful comments to improve the presentation in the paper.

\section{Basic notions}

In this section, we recall some basic notions on bornological modules and bounded homomorphisms. See $\mathbf{1 1}$ for more details. We also study the inheritance properties of separatedness and completeness for submodules, quotients and extensions.

A bornology on a set $X$ is a collection $\mathcal{B}_{X}$ of subsets of $X$, called bounded sets, such that all finite subsets are bounded and subsets and finite unions of bounded subsets are bounded. Let $V$ be a complete discrete valuation ring. A bornological $V$-module is a $V$-module $M$ with a bornology such that every bounded subset is 
contained in a bounded $V$-submodule. In particular, the $V$-submodule generated by a bounded subset is again bounded. We always write $\mathcal{B}_{M}$ for the bornology on $M$.

Let $M^{\prime} \subseteq M$ be a $V$-submodule. The subspace bornology on $M^{\prime}$ consists of all subsets of $M^{\prime}$ that are bounded in $M$. The quotient bornology on $M / M^{\prime}$ consists of all subsets of the form $q(S)$ with $S \in \mathcal{B}_{M}$, where $q: M \rightarrow M / M^{\prime}$ is the canonical projection. We always equip submodules and quotients with these canonical bornologies.

Let $M$ and $N$ be two bornological $V$-modules. A $V$-module map $f: M \rightarrow N$ is bounded if $f(S) \in \mathcal{B}_{N}$ for all $S \in \mathcal{B}_{M}$. Bornological $V$-modules and bounded $V$-module maps form an additive category. The isomorphisms in this category are called bornological isomorphisms. A bounded $V$-module map $f: M \rightarrow N$ is a bornological embedding if the induced map $M \rightarrow f(M)$ is a bornological isomorphism, where $f(M) \subseteq N$ carries the subspace bornology. It is a bornological quotient map if the induced map $M / \operatorname{ker} f \rightarrow N$ is a bornological isomorphism. Equivalently, for each $T \in \mathcal{B}_{N}$ there is $S \in \mathcal{B}_{M}$ with $f(S)=T$.

An extension of bornological $V$-modules is a diagram of $V$-modules

$$
M^{\prime} \stackrel{f}{\rightarrow} M \stackrel{g}{\rightarrow} M^{\prime \prime}
$$

that is algebraically exact and such that $f$ is a bornological embedding and $g$ a bornological quotient map. Equivalently, $g$ is a cokernel of $f$ and $f$ a kernel of $g$ in the additive category of bornological $V$-modules. A split extension is an extension with a bounded $V$-linear map $s: M^{\prime \prime} \rightarrow M$ such that $g \circ s=\operatorname{id}_{M^{\prime \prime}}$.

Let $M$ be a bornological $V$-module. A sequence $\left(x_{n}\right)_{n \in \mathbb{N}}$ in $M$ converges towards $x \in M$ if there are $S \in \mathcal{B}_{M}$ and a sequence $\left(\delta_{n}\right)_{n \in \mathbb{N}}$ in $V$ with $\lim \left|\delta_{n}\right|=0$ and $x_{n}-x \in \delta_{n} \cdot S$ for all $n \in \mathbb{N}$. It is a Cauchy sequence if there are $S \in \mathcal{B}_{M}$ and a sequence $\left(\delta_{n}\right)_{n \in \mathbb{N}}$ in $V$ with $\lim \left|\delta_{n}\right|=0$ and $x_{n}-x_{m} \in \delta_{j} \cdot S$ for all $n, m, j \in \mathbb{N}$ with $n, m \geq j$. Since any bounded subset is contained in a bounded $V$-submodule, a sequence in $M$ converges or is Cauchy if and only if it converges or is Cauchy in the $\pi$-adic topology on some bounded $V$-submodule of $M$.

We call a subset $S$ of $M$ closed if $x \in S$ for any sequence in $S$ that converges in $M$ to $x \in M$. These are the closed subsets of a topology on $M$. Bounded maps preserve convergent sequences and Cauchy sequences. Thus pre-images of closed subsets under bounded maps remain closed. That is, bounded maps are continuous for these canonical topologies.

2.1. Separated bornological modules. We call $M$ separated if limits of convergent sequences in $M$ are unique. If $M$ is not separated, then the constant sequence 0 has a non-zero limit. Therefore, $M$ is separated if and only if $\{0\} \subseteq M$ is closed. And $M$ is separated if and only if any $S \in \mathcal{B}_{M}$ is contained in a $\pi$-adically separated bounded $V$-submodule.

Lemma 2.1. Let $M^{\prime} \stackrel{f}{\rightarrow} M \stackrel{g}{\rightarrow} M^{\prime \prime}$ be an extension of bornological $V$-modules.

(1) If $M$ is separated, so is $M^{\prime}$.

(2) The quotient $M^{\prime \prime}$ is separated if and only if $f\left(M^{\prime}\right)$ is closed in $M$.

(3) If $M^{\prime}$ and $M^{\prime \prime}$ are separated and $M^{\prime \prime}$ is torsion-free, then $M$ is separated.

Proof. Assertion (1) is trivial.

If $M^{\prime \prime}$ is separated, then $\{0\} \subseteq M^{\prime \prime}$ is closed. Hence $g^{-1}(\{0\})=f\left(M^{\prime}\right)$ is closed in $M$. If $M^{\prime \prime}$ is not separated, then the constant sequence 0 in $M^{\prime \prime}$ converges to some non-zero $x^{\prime \prime} \in M^{\prime \prime}$. That is, there are a bounded subset $S^{\prime \prime} \subseteq M^{\prime \prime}$ and a null 
sequence $\left(\delta_{n}\right)_{n \in \mathbb{N}}$ in $V$ with $x^{\prime \prime}-0 \in \delta_{n} \cdot S^{\prime \prime}$ for all $n \in \mathbb{N}$. Since $g$ is a bornological quotient map, there are $x \in M$ and $S \in \mathcal{B}_{M}$ with $g(x)=x^{\prime \prime}$ and $g(S)=S^{\prime \prime}$. We may choose $y_{n}^{\prime \prime} \in S^{\prime \prime}$ with $x^{\prime \prime}=\delta_{n} \cdot y_{n}^{\prime \prime}$ and $y_{n} \in S$ with $g\left(y_{n}\right)=y_{n}^{\prime \prime}$. So $g\left(x-\delta_{n} y_{n}\right)=0$. Thus the sequence $\left(x-\delta_{n} y_{n}\right)$ lies in $f\left(M^{\prime}\right)$. It converges to $x$, which does not belong to $f\left(M^{\prime}\right)$ because $x^{\prime \prime} \neq 0$. So $f\left(M^{\prime}\right)$ is not closed. This finishes the proof of (2)

We prove (3) Let $x \in M$ belong to the closure of $\{0\}$ in $M$. That is, there are $S \in \mathcal{B}_{M}$ and a null sequence $\left(\delta_{n}\right)_{n \in \mathbb{N}}$ in $V$ with $x \in \delta_{n} \cdot S$ for all $n \in \mathbb{N}$. Then $g(x) \in \delta_{n} \cdot g(S)$ for all $n \in \mathbb{N}$. This implies $g(x)=0$ because $M^{\prime \prime}$ is separated. So there is $y \in M^{\prime}$ with $f(y)=x$. And $f(y)=x \in \delta_{n} \cdot S$. Choose $x_{n} \in S$ with $f(y)=\delta_{n} \cdot x_{n}$. We may assume $\delta_{n} \neq 0$ for all $n \in \mathbb{N}$ because otherwise $x \in \delta_{n} \cdot S$ is 0 . Since $M^{\prime \prime}$ is torsion-free, $\delta_{n} \cdot x_{n} \in f\left(M^{\prime}\right)$ implies $g\left(x_{n}\right)=0$. So we may write $x_{n}=f\left(y_{n}\right)$ for some $y_{n} \in M^{\prime}$. Since $f$ is a bornological embedding, the set $\left\{y_{n}: n \in \mathbb{N}\right\}$ in $M^{\prime}$ is bounded. Since $M^{\prime}$ is separated and $y=\delta_{n} \cdot y_{n}$ for all $n \in \mathbb{N}$, we get $y=0$. Hence $x=0$. So $\{0\}$ is closed in $M$.

The quotient $M / \overline{\{0\}}$ of a bornological $V$-module $M$ by the closure of 0 is called the separated quotient of $M$. It is separated by Lemma 2.1. and it is the largest separated quotient of $M$. Even more, the quotient map $M \rightarrow M / \overline{\{0\}}$ is the universal arrow to a separated bornological $V$-module, that is, any bounded $V$-linear map from $M$ to a separated bornological $V$-module factors uniquely through $M / \overline{\{0\}}$.

The following example shows that Lemma 2.1[3) fails without the torsionfreeness assumption.

ExAmple 2.2. Let $M^{\prime}=V$ and let $M=V[x] / S$, where $S$ is the $V$-submodule of $V[x]$ generated by $1-\pi^{n} x^{n}$ for all $n \in \mathbb{N}$. We embed $M^{\prime}=V$ as multiples of $1=x^{0}$. Then

$$
M / M^{\prime}=\bigoplus_{n=1}^{\infty} V /\left(\pi^{n}\right),
$$

We endow $M, M^{\prime}$ and $M / M^{\prime}$ with the bornologies where all subsets are bounded. We get an extension of bornological $V$-modules $V \rightarrow M \rightarrow \oplus_{n=1}^{\infty} V /\left(\pi^{n}\right)$. Here $V$ and $\oplus_{n=1}^{\infty} V /\left(\pi^{n}\right)$ are $\pi$-adically separated, but $M$ is not: the constant sequence 1 in $M$ converges to 0 because $1=1-\pi^{n} x^{n}+\pi^{n} x^{n} \equiv \pi^{n} x^{n}$ in $M$.

2.2. Completeness. We call a bornological $V$-module $M$ complete if it is separated and for any $S \in \mathcal{B}_{M}$ there is $T \in \mathcal{B}_{M}$ so that all $S$-Cauchy sequences are $T$-convergent. Equivalently, any $S \in \mathcal{B}_{M}$ is contained in a $\pi$-adically complete bounded $V$-submodule (see [11. Proposition 2.8]). By definition, any Cauchy sequence in a complete bornological $V$-module has a unique limit.

THEOREM 2.3. Let $M^{\prime} \stackrel{f}{\rightarrow} M \stackrel{g}{\rightarrow} M^{\prime \prime}$ be an extension of bornological $V$-modules.

(1) If $M$ is complete and $f\left(M^{\prime}\right)$ is closed in $M$, then $M^{\prime}$ is complete.

(2) If $M^{\prime}$ is complete, $M$ separated, and $M^{\prime \prime}$ torsion-free, then $f\left(M^{\prime}\right)$ is closed in $M$.

(3) Let $M$ be complete. Then $M^{\prime \prime}$ is complete if and only if $f\left(M^{\prime}\right)$ is closed in $M$.

(4) If $M^{\prime}$ and $M^{\prime \prime}$ are complete and $M$ is separated, then $M$ is complete. If $M^{\prime}$ and $M^{\prime \prime}$ are complete and $M^{\prime \prime}$ is torsion-free, then $M$ is complete.

Proof. Statement (1) is 11. Lemma 2.13], and there is no need to repeat the proof here. It is somewhat similar to the proof of (4) Next we prove (2). Assume 
that $M^{\prime}$ is complete, that $M^{\prime \prime}$ is torsion-free, and that $f\left(M^{\prime}\right)$ is not closed in $M$. We are going to prove that $M$ is not separated. There is a sequence $\left(x_{n}\right)_{n \in \mathbb{N}}$ in $M^{\prime}$ for which $f\left(x_{n}\right)_{n \in \mathbb{N}}$ converges in $M$ towards some $x \notin f\left(M^{\prime}\right)$. So there is a bounded set $S \subseteq M$ and a sequence $\left(\delta_{k}\right)_{k \in \mathbb{N}}$ in $V$ with $\lim \left|\delta_{k}\right|=0$ and $f\left(x_{n}\right)-x \in \delta_{n} \cdot S$ for all $n \in \mathbb{N}$. We may assume without loss of generality that $S$ is a bounded $V$-submodule and that the sequence of norms $\left|\delta_{n}\right|$ is decreasing: let $\delta_{n}^{*}$ be the $\delta_{m}$ for $m \geq n$ with maximal norm. Then $f\left(x_{n}\right)-x \in \delta_{n} \cdot S \subseteq \delta_{n}^{*} \cdot S$ and still $\lim \left|\delta_{n}^{*}\right|=0$. We may write $f\left(x_{n}\right)-x=\delta_{n}^{*} y_{n}$ with $y_{n} \in S$. Let $m<n$. Then $\delta_{m}^{*} g\left(y_{m}\right)=-g(x)=$ $\delta_{n}^{*} g\left(y_{n}\right)$ and $\delta_{n}^{*} / \delta_{m}^{*} \in V$; this implies first $\delta_{m}^{*} \cdot\left(g\left(y_{m}\right)-g\left(y_{n}\right) \cdot \delta_{n}^{*} / \delta_{m}^{*}\right)=0$ and then $g\left(y_{m}\right)=g\left(y_{n}\right) \cdot \delta_{n}^{*} / \delta_{m}^{*}$ because $M^{\prime \prime}$ is torsion-free. So there is $z_{m, n} \in M^{\prime}$ with $y_{m}+f\left(z_{m, n}\right)=y_{n} \cdot \delta_{n}^{*} / \delta_{m}^{*}$. We even have $z_{m, n} \in f^{-1}(S)$ because $S$ is a $V$-submodule. The subset $f^{-1}(S) \subseteq M^{\prime}$ is bounded because $f$ is a bornological embedding. We get $f\left(x_{n}\right)-f\left(x_{m}\right)=\delta_{n}^{*} y_{n}-\delta_{m}^{*} y_{m}=f\left(\delta_{m}^{*} z_{m, n}\right)$ and hence $x_{n}-x_{m}=\delta_{m}^{*} z_{m, n}$ for $n>m$. This witnesses that the sequence $\left(x_{n}\right)_{n \in \mathbb{N}}$ is Cauchy in $M^{\prime}$. Since $M^{\prime}$ is complete, it converges towards some $y \in M^{\prime}$. Then $f\left(x_{n}\right)$ converges both towards $f(y) \in f\left(M^{\prime}\right)$ and towards $x \notin f\left(M^{\prime}\right)$. So $M$ is not separated. This finishes the proof of (2).

Next we prove (3) If $f\left(M^{\prime}\right)$ is not closed, then Lemma 2.1 shows that $M^{\prime \prime}$ is not separated and hence not complete. Conversely, we claim that $M^{\prime \prime}$ is complete if $f\left(M^{\prime}\right)$ is closed. Lemma 2.1 shows that $M^{\prime \prime}$ is separated. Let $S^{\prime \prime} \in \mathcal{B}_{M^{\prime \prime}}$. There is $S \in \mathcal{B}_{M}$ with $g(S)=S^{\prime \prime}$ because $g$ is a bornological quotient map. And there is $T \in \mathcal{B}_{M}$ so that any $S$-Cauchy sequence is $T$-convergent. We claim that any $S^{\prime \prime}$-Cauchy sequence is $g(T)$-convergent. So let $\left(x_{n}^{\prime \prime}\right)_{n \in \mathbb{N}}$ be an $S^{\prime \prime}$-Cauchy sequence. Thus there is a null sequence $\left(\delta_{n}\right)_{n \in \mathbb{N}}$ in $V$ with $x_{n}^{\prime \prime}-x_{m}^{\prime \prime} \in \delta_{j} \cdot S^{\prime \prime}$ for all $n, m, j \in \mathbb{N}$ with $n, m \geq j$. As above, we may assume without loss of generality that the sequence of norms $\left|\delta_{n}\right|$ is decreasing. Choose any $x_{0} \in M$ with $g\left(x_{0}\right)=x_{0}^{\prime \prime}$. For each $n \in \mathbb{N}$, choose $y_{n} \in S$ with $x_{n+1}^{\prime \prime}-x_{n}^{\prime \prime}=\delta_{n} \cdot g\left(y_{n}\right)$. Let

$$
x_{n}:=x_{0}+\delta_{0} \cdot y_{0}+\cdots+\delta_{n-1} \cdot y_{n-1} .
$$

Then $g\left(x_{n}\right)=x_{n}^{\prime \prime}$. And $x_{n+1}-x_{n}=\delta_{n} \cdot y_{n} \in \delta_{n} \cdot S$. Since $\left|\delta_{n}\right|$ is decreasing, this implies $x_{m}-x_{n} \in \delta_{n} \cdot S$ for all $m \geq n$. So the sequence $\left(x_{n}\right)_{n \in \mathbb{N}}$ is $S$-Cauchy. Hence it is $T$-convergent. Thus $g\left(x_{n}\right)=x_{n}^{\prime \prime}$ is $g(T)$-convergent as asserted. This finishes the proof of (3)

Finally, we prove (4). So we assume $M^{\prime}$ and $M^{\prime \prime}$ to be complete. If $M^{\prime \prime}$ is torsion-free, then $M$ is separated by Lemma 2.1. Hence the second statement in (4) is a special case of the first one. Let $S \in \mathcal{B}_{M}$. We must find $T \in \mathcal{B}_{M}$ so that every $S$-Cauchy sequence is $T$-convergent. Since $M$ is separated, this says that it is complete. Since $M^{\prime \prime}$ is complete, there is a $\pi$-adically complete $V$-submodule $T_{0} \in \mathcal{B}_{M^{\prime \prime}}$ that contains $g(S)$. Since $g$ is a bornological quotient map, there is $T_{1} \in \mathcal{B}_{M}$ with $g\left(T_{1}\right)=T_{0}$. Replacing it by $T_{1}+S$, we may arrange, in addition, that $S \subseteq T_{1}$. Since $f$ is a bornological embedding, $T_{2}:=f^{-1}\left(T_{1}\right)$ is bounded in $M^{\prime}$. As $M^{\prime}$ is complete, there is $T_{3} \in \mathcal{B}_{M^{\prime}}$ so that every $T_{2}$-Cauchy sequence is $T_{3}$-convergent. We claim that any $S$-Cauchy sequence is $T_{1}+f\left(T_{3}\right)$-convergent. The proof of this claim will finish the proof of the theorem.

Let $\left(x_{n}\right)_{n \in \mathbb{N}}$ be an $S$-Cauchy sequence. So there are $\delta_{n} \in V$ and $y_{n} \in S$ with $\lim \left|\delta_{n}\right|=0$ and $x_{n+1}-x_{n}=\delta_{n} \cdot y_{n}$. As above, we may assume that $\left|\delta_{n}\right|$ is decreasing and that $\delta_{0}=1$. Since $g\left(y_{n+k}\right) \in g(S) \subseteq T_{0}$ and $T_{0}$ is $\pi$-adically complete, the 
following series converges in $T_{0}$ :

$$
\tilde{w}_{n}:=-\sum_{k=0}^{\infty} \frac{\delta_{n+k}}{\delta_{n}} g\left(y_{n+k}\right) .
$$

Since $\tilde{w}_{n} \in T_{0}$, there is $w_{n} \in T_{1}$ with $g\left(w_{n}\right)=\tilde{w}_{n}$. So

$$
\begin{aligned}
\delta_{n} g\left(w_{n}\right)=\lim _{N \rightarrow \infty}-g\left(\sum_{k=0}^{N} \delta_{n+k} y_{n+k}\right) & \\
& =\lim _{N \rightarrow \infty} g\left(x_{n}-x_{N+n+1}\right)=g\left(x_{n}\right)-\lim _{N \rightarrow \infty} g\left(x_{N}\right) .
\end{aligned}
$$

In particular, $g\left(w_{0}\right)=g\left(x_{0}\right)-\lim _{N \rightarrow \infty} g\left(x_{N}\right)$. Now let

$$
\tilde{x}_{k}:=x_{k}-\delta_{k} w_{k}+w_{0}-x_{0} \text {. }
$$

Then

$$
g\left(\tilde{x}_{k}\right)=g\left(x_{k}\right)-g\left(x_{k}\right)+\lim _{N \rightarrow \infty} g\left(x_{N}\right)+g\left(x_{0}\right)-\lim _{N \rightarrow \infty} g\left(x_{N}\right)-g\left(x_{0}\right)=0 .
$$

So $\tilde{x}_{k} \in f\left(M^{\prime}\right)$ for all $k \in \mathbb{N}$. And

$$
\begin{aligned}
\tilde{x}_{n+1}-\tilde{x}_{n}=x_{n+1}- & x_{n}-\delta_{n+1} w_{n+1}+\delta_{n} w_{n} \\
& =\delta_{n} y_{n}+\delta_{n} w_{n}-\delta_{n+1} w_{n+1}=\delta_{n} \cdot\left(y_{n}+w_{n}-\frac{\delta_{n+1}}{\delta_{n}} w_{n+1}\right) .
\end{aligned}
$$

Let $z_{n}:=y_{n}+w_{n}-\frac{\delta_{n+1}}{\delta_{n}} w_{n+1}$. A telescoping sum argument shows that

$$
g\left(z_{n}\right)=g\left(y_{n}\right)+\tilde{w}_{n}-\frac{\delta_{n+1}}{\delta_{n}} \tilde{w}_{n+1}=0 .
$$

So $z_{n} \in f\left(M^{\prime}\right)$. And $z_{n} \in S+T_{1}+T_{1}=T_{1}$. Thus there is $\hat{z}_{n} \in f^{-1}\left(T_{1}\right)=T_{2}$ with $z_{n}=f\left(\hat{z}_{n}\right)$. Equation 2.5 means that the sequence $f^{-1}\left(\tilde{x}_{n}\right)$ is $T_{2}$-Cauchy. Hence it is $T_{3}$-convergent. So $\left(\widetilde{x}_{n}\right)$ is $f\left(T_{3}\right)$-convergent. Then $\left(x_{n}\right)$ is $T_{1}+f\left(T_{3}\right)$ convergent.

The following examples show that the technical extra assumptions in (2) and (4) in Theorem 2.3 are necessary. They only involve extensions of $V$-modules with the bornology where all subsets are bounded. For this bornology, bornological completeness and separatedness are the same as $\pi$-adic completeness and separatedness, respectively, and any extension of $V$-modules is a bornological extension.

ExAMPLE 2.7. Let $M^{\prime}:=\{0\}$ and $M:=F$ with the bornology of all subsets. Then $M^{\prime}$ is bornologically complete, but not closed in $M$, and $M / M^{\prime}=M$ is torsion-free. So Theorem 2.3(2) needs the assumption that $M$ be separated.

EXAMPLE 2.8. Let $M$ be the $V$-module of all power series $\sum_{n=0}^{\infty} c_{n} x^{n}$ with $\lim \left|c_{n}\right|=0$ and with the bornology where all subsets are bounded; this is the $\pi$-adic completion of the polynomial algebra $V[x]$. Let $M^{\prime}=M$ and define $f: M^{\prime} \rightarrow M$, $f\left(\sum_{n=0}^{\infty} c_{n} x^{n}\right):=\sum_{n=0}^{\infty} c_{n} \pi^{n} x^{n}$. This is a bornological embedding simply because all subsets in $M=M^{\prime}$ are bounded. Let $p_{n}:=\sum_{j=0}^{n} x^{j}$. This sequence in $M^{\prime}=M$ does not converge. Nevertheless, the sequence $f\left(p_{n}\right)=\sum_{j=0}^{n} \pi^{j} x^{j}$ converges in $M$ to $\sum_{j=0}^{\infty} \pi^{j} x^{j}$. Thus $f\left(M^{\prime}\right)$ is not closed in $M$, although $M$ and $M^{\prime}$ are complete and $f$ is a bornological embedding. So Theorem 2.3 (2) needs the assumption that $M^{\prime \prime}$ be torsion-free. 
EXAmple 2.9. We modify Example 2.2 to produce an extension of $V$-modules $N^{\prime} \rightarrow N \rightarrow N^{\prime \prime}$ where $N^{\prime}$ and $N^{\prime \prime}$ are $\pi$-adically complete, but $N$ is not $\pi$-adically separated and hence not $\pi$-adically complete. We let $N^{\prime}:=V /(\pi)=\mathbb{F}$. We let $N^{\prime \prime}$ be the $\pi$-adic completion of the $V$-module $M^{\prime \prime}$ of Example 2.2 That is,

$$
N^{\prime \prime}:=\left\{\left(c_{n}\right)_{n \in \mathbb{N}} \in \prod_{n=0}^{\infty} V /\left(\pi^{n}\right): \lim \left|c_{n}\right|=0\right\} .
$$

This is indeed $\pi$-adically complete. So is

$$
N_{1}:=\left\{\left(c_{n}\right)_{n \in \mathbb{N}} \in \prod_{n=0}^{\infty} V /\left(\pi^{n+1}\right): \lim \left|c_{n}\right|=0\right\} .
$$

The kernel of the quotient map $q: N_{1} \rightarrow N^{\prime \prime}$ is isomorphic to $\prod_{n=0}^{\infty} V /(\pi)=\prod_{\mathbb{N}} \mathbb{F}$. This is a $\mathbb{F}$-vector space, and it contains the $\mathbb{F}$-vector space $\sum_{n=0}^{\infty} \mathbb{F}$. Since any $\mathbb{F}$-vector space has a basis, we may extend the linear functional $\sum_{n=0}^{\infty} \mathbb{F} \rightarrow \mathbb{F},\left(c_{n}\right)_{n \in \mathbb{N}} \mapsto$ $\sum_{n=0}^{\infty} c_{n}$, to a $\mathbb{F}$-linear functional $\sigma: \prod_{\mathbb{N}} \mathbb{F} \rightarrow \mathbb{F}$. Let $L:=\operatorname{ker} \sigma \subseteq \operatorname{ker} q$ and let $N:=N_{1} / L$. The map $q$ descends to a surjective $\pi$-linear map $N \rightarrow N^{\prime \prime}$. Its kernel is isomorphic to $\prod_{\mathbb{N}} \mathbb{F} / \operatorname{ker} \sigma \cong \mathbb{F}=N^{\prime}$. The functional $\sigma: \prod_{\mathbb{N}} \mathbb{F} \rightarrow \mathbb{F}$ vanishes on $\delta_{0}-\delta_{k}$ for all $k \in \mathbb{N}$, but not on $\delta_{0}$. When we identify $\Pi_{\mathbb{N}} \mathbb{F} \cong \operatorname{ker} q$, we map $\delta_{k}$ to $\pi^{k} \delta_{k} \in N_{1}$. So $\delta_{0}$ and $\pi^{k} \delta_{k}$ get identified in $N$, but $\delta_{0}$ does not become 0 : it is the generator of $N^{\prime}=V /(\pi)$ inside $N$. Since $\left[\delta_{0}\right]=\pi^{k}\left[\delta_{k}\right]$ in $N$, the $V$-module $N$ is not $\pi$-adically separated.

The completion $\overparen{M}$ of a bornological $V$-module $M$ is a complete bornological $V$-module with a bounded $V$-linear map $M \rightarrow T$ that is universal in the sense that any bounded $V$-linear map from $M$ to a complete bornological $V$-module $X$ factors uniquely through $M$. Such a completion exists and is unique up to isomorphism (see [11, Proposition 2.15]). We shall describe it more concretely later when we need the details of its construction.

2.3. Vector spaces over the fraction field. Recall that $F$ denotes the quotient field of $V$. Any $V$-linear map between two $F$-vector spaces is also $F$-linear. So $F$-vector spaces with $F$-linear maps form a full subcategory in the category of $V$-modules. A $V$-module $M$ comes from a $F$-vector space if and only if the map

$$
\pi_{M}: M \rightarrow M, \quad m \mapsto \pi \cdot m,
$$

is invertible. We could define bornological $F$-vector spaces without reference to $V$. Instead, we realise them as bornological $V$-modules with an extra property:

Definition 2.11. A bornological $V$-module $M$ is a bornological $F$-vector space if the map $\pi_{M}$ in 2.10 is a bornological isomorphism, that is, an invertible map with bounded inverse.

Given a bornological $V$-module $M$, the tensor product $F \otimes M:=F \otimes_{V} M$ with the tensor product bornology (see [11 Lemma 2.18]) is a bornological $F$-vector space because multiplication by $\pi$ is a bornological isomorphism on $F$.

LEMma 2.12. The canonical bounded $V$-linear map

$$
\iota_{M}: M \rightarrow F \otimes M, \quad m \mapsto 1 \otimes m,
$$

is the universal arrow from $M$ to a bornological $F$-vector space, that is, any bounded $V$-linear map $f: M \rightarrow N$ to a bornological F-vector space $N$ factors uniquely through a bounded $V$-linear map $f^{\#}: F \otimes M \rightarrow N$, and this map is also $F$-linear. 
Proof. A $V$-linear map $f^{\#}: F \otimes M \rightarrow N$ must be $F$-linear. Hence the only possible candidate is the $F$-linear map defined by $f^{\#}(x \otimes m):=x \cdot f(m)$ for $m \in M$, $x \in F$. Any bounded submodule of $F \otimes M$ is contained in $\pi^{-k} V \otimes S$ for some bounded submodule $S \subseteq M$ and some $k \in \mathbb{N}$, and $f^{\#}\left(\pi^{-k} V \otimes S\right)=\pi_{N}^{-k}(f(S))$ is bounded in $N$ because $\pi_{N}$ is a bornological isomorphism. Thus $f^{\#}$ is bounded.

\section{Spectral radius and semi-dagger algebras}

A bornological $V$-algebra is a bornological $V$-module $A$ with a bounded, $V$-linear, associative multiplication. We do not assume $A$ to have a unit element. We fix a bornological $V$-algebra $A$ throughout this section.

We recall some definitions from $[\mathbf{1 1}]$. Let $\varepsilon=|\pi|$. Let $S \in \mathcal{B}_{A}$ and let $r \leq 1$. There is a smallest integer $j$ with $\varepsilon^{j} \leq r$, namely, $\left\lceil\log _{\varepsilon}(r)\right\rceil$. Define

$$
r \star S:=\pi^{\left\lceil\log _{\varepsilon}(r)\right\rceil} \cdot S .
$$

Let $\sum_{n=1}^{\infty} r^{n} \star S^{n}$ be the $V$-submodule generated by $\bigcup_{n=1}^{\infty} r^{n} \star S^{n}$. That is, its elements are finite $V$-linear combinations of elements in $\bigcup_{n=1}^{\infty} r^{n} \star S^{n}$.

Definition 3.1. The truncated spectral radius $\varrho_{1}(S)=\varrho_{1}\left(S ; \mathcal{B}_{A}\right)$ of $S \in \mathcal{B}_{A}$ is the infimum of all $r \geq 1$ for which $\sum_{n=1}^{\infty} r^{-n} \star S^{n}$ is bounded. It is $\infty$ if no such $r$ exists.

By definition, $\varrho_{1}(S) \in[1, \infty]$. If $A$ is an algebra over the fraction field $F$ of $V$, then we may define $\sum_{n=1}^{\infty} r^{-n} \star S^{n}$ also for $0<r<1$. Then the full spectral radius $\varrho(S) \in[0, \infty]$ is defined like $\varrho_{1}(S)$, but without the restriction to $r \geq 1$. The arguments in the beginning of Section 3.1 in $[\mathbf{1 1}$ assume implicitly that the $V$-algebra $A$ is a bornological subalgebra in a $F$-algebra, so that the spectral radius is defined without truncation. This means that $A$ is bornologically torsion-free (see Proposition 4.3. . The results in [11. Section 3.1] work without this assumption if the truncated spectral radius is used throughout and the following lemma is used instead of [11, Lemma 3.1.2]:

Lemma 3.2. Let $S \subseteq A$ be a bounded $V$-submodule and $m \in \mathbb{N}_{\geq 1}$. Then $\varrho_{1}(S)=1$ if and only if $\sum_{l=1}^{\infty}\left(\pi^{m} S^{j}\right)^{l}$ is bounded for all $j \in \mathbb{N}_{\geq 1}$.

Proof. Let $\varrho_{1}(S)=1$ and $j \in \mathbb{N}_{\geq 1}$. Then $\sum_{l=1}^{\infty} \pi^{m \cdot l} S^{j \cdot l} \subseteq \sum_{k=1}^{\infty}\left(\varepsilon^{m / j}\right)^{k} \star S^{k}$ is bounded because $\varepsilon^{m / j}<1$. Conversely, let $\sum_{l=1}^{\infty}\left(\pi^{m} S^{j}\right)^{l}$ be bounded. Then $\varrho_{1}\left(\pi^{m} S^{j}\right)=1$. The proof of $\left[\mathbf{1 1}\right.$. Lemma 3.1.2] shows that $\varrho_{1}(S) \leq \varepsilon^{-m / j}$. This inequality for all $j \in \mathbb{N}_{\geq 1}$ implies $\varrho_{1}(S)=1$.

Definition 3.3. A bornological $V$-algebra $A$ is semi-dagger if $\varrho_{1}(S)=1$ for all $S \in \mathcal{B}_{A}$.

Proposition 3.4 ([11. Proposition 3.1.3]). A bornological V-algebra $A$ is semidagger if and only if $\sum_{i=0}^{\infty} \pi^{i} S^{i+1}$ is bounded for all $S \in \mathcal{B}_{A}$, if and only if $\sum_{i=0}^{\infty} \pi^{i} S^{c i+d}$ is bounded for all $S \in \mathcal{B}_{A}$ and $c, d \in \mathbb{N}$ with $d \geq 1$, if and only if any $S \in \mathcal{B}_{A}$ is contained in a bounded $V$-submodule $U \subseteq A$ with $\pi \cdot U \cdot U \subseteq U$.

Definition 3.5. The linear growth bornology on a bornological $V$-algebra $A$ is the smallest semi-dagger bornology on $A$. That is, it is the smallest bornology $\mathcal{B}_{A}^{\prime}$ with $\varrho_{1}\left(S ; \mathcal{B}_{A}^{\prime}\right)=1$ for all $S \in \mathcal{B}_{A}^{\prime}$. Let $A_{\lg }$ be $A$ with the linear growth bornology.

The existence of a smallest semi-dagger bornology is shown in $\mathbf{1 1}$ by describing it explicitly as follows: 
Lemma 3.6 ([11, Proposition 3.1.3 and Lemma 3.1.10]). Let $T \subseteq A$. The following are equivalent:

(1) $T$ is bounded in $A_{\mathrm{lg}}$;

(2) $T \subseteq \sum_{i=0}^{\infty} \pi^{i} S^{i+1}$ for some $S \in \mathcal{B}_{A}$.

(3) $T \subseteq \sum_{i=0}^{\infty} \pi^{i} S^{c i+d}$ for some $S \in \mathcal{B}_{A}$ and $c, d \in \mathbb{N}$ with $d \geq 1$.

More precisely, the proof of [11, Lemma 3.1.10] shows that the subsets in (2) form a semi-dagger bornology that contains $\mathcal{B}_{A}$. And Proposition 3.4 shows that these subsets are bounded in any semi-dagger bornology on $A$ that contains $\mathcal{B}_{A}$. So they form the smallest semi-dagger bornology containing $\mathcal{B}_{A}$.

By definition, the algebra $A_{\lg }$ has the following universal property: if $B$ is a semi-dagger $V$-algebra, then an algebra homomorphism $A \rightarrow B$ is bounded if and only if it is bounded on $A_{\mathrm{lg}}$. The algebra $A$ is semi-dagger if and only if $A=A_{\mathrm{lg}}$.

THEOREM 3.7. Let $A \stackrel{i}{\rightarrow} B \stackrel{q}{\rightarrow} C$ be an extension of bornological $V$-algebras. Then $B$ is a semi-dagger algebra if and only if both $A$ and $C$ are.

Proof. First assume $B$ to be semi-dagger. Let $S \in \mathcal{B}_{A}$. Then $\sum_{j=0}^{\infty} \pi^{j} i(S)^{j+1}$ is bounded in $B$. Since $i$ is a bornological embedding, it follows that $\sum_{j=0}^{\infty} \pi^{j} S^{j+1}$ is bounded in $A$. That is, $\varrho_{1}\left(S ; \mathcal{B}_{A}\right)=1$. So $A$ is semi-dagger. Now let $S \in \mathcal{B}_{C}$. Since $q$ is a bornological quotient map, there is $T \in \mathcal{B}_{B}$ with $q(T)=S$. The subset $\sum_{j=0}^{\infty} \pi^{j} T^{j+1}$ is bounded in $B$ because $B$ is semi-dagger. Its image under $q$ is also bounded, and this is $\sum_{j=0}^{\infty} \pi^{j} S^{j+1}$. So $\varrho_{1}\left(S ; \mathcal{B}_{C}\right)=1$ and $C$ is semi-dagger.

Now assume that $A$ and $C$ are semi-dagger. We show that $\sum_{l=1}^{\infty}\left(\pi^{2} S^{j}\right)^{l}$ is bounded in $B$ for all $S \in \mathcal{B}_{B}, j \in \mathbb{N}_{\geq 1}$. This implies $\varrho_{1}\left(S ; \mathcal{B}_{B}\right)=1$ by Lemma 3.2

Since $C$ is semi-dagger, $\varrho_{1}\left(q(S) ; \mathcal{B}_{C}\right)=1$. Thus $S_{2}:=\sum_{l=1}^{\infty} q\left(\pi S^{j}\right)^{l}$ is bounded in $C$ by Lemma 3.2 Since $q$ is a quotient map, there is $T \in \mathcal{B}_{B}$ with $q(T)=S_{2}$. We may choose $T$ with $\pi S^{j} \subseteq T$. For each $x, y \in T$, we have $q(x \cdot y) \in S_{2} \cdot S_{2} \subseteq S_{2}=q(T)$. Hence there is $\omega(x, y) \in T$ with $x \cdot y-\omega(x, y) \in i(A)$. Let

$$
\Omega:=\{x \cdot y-\omega(x, y): x, y \in T\} .
$$

This is contained in $T^{2}-T$. So $\Omega \in \mathcal{B}_{B}$. And $T^{2} \subseteq T+\Omega$. By construction, $\Omega$ is also contained in $i(A)$. Since $i$ is a bornological embedding, $i^{-1}(\Omega)$ is bounded in $A$. Since $A$ is semi-dagger, we have $\varrho_{1}\left(i^{-1}(\Omega) ; \mathcal{B}_{A}\right)=1$. So $\sum_{n=1}^{\infty}(\pi \cdot \Omega)^{n}$ is bounded. Thus the subset

$$
U:=\sum_{n=1}^{\infty}(\pi \cdot \Omega)^{n}+\sum_{n=0}^{\infty} T \cdot(\pi \cdot \Omega)^{n}=\sum_{n=1}^{\infty}(\pi \cdot \Omega)^{n}+T+\sum_{n=1}^{\infty} T \cdot(\pi \cdot \Omega)^{n}
$$

of $B$ is bounded. Using $T^{2} \subseteq T+\Omega$, we prove that $\pi T \cdot U \subseteq U$. Hence $(\pi T)^{n} \cdot U \subseteq U$ for all $n \in \mathbb{N}_{\geq 1}$ by induction. Since $T \subseteq U$, this implies $\sum_{l=1}^{\infty} \pi^{l} T^{l+1} \subseteq U$. Hence $\sum_{l=2}^{\infty}(\pi T)^{l}=\pi \cdot \sum_{l=1}^{\infty} \pi^{l} T^{l+1} \subseteq \pi U$. Therefore, $\sum_{l=1}^{\infty}(\pi T)^{l}$ is bounded. Since $\pi^{2} S^{j} \subseteq \pi T$, it follows that $\sum_{l=1}^{\infty}\left(\pi^{2} S^{j}\right)^{l}$ is bounded for all $j$, as desired.

Proposition 3.8 (11, Lemma 3.1.12]). If $A$ is semi-dagger, then so is its completion $\bar{A}$.

Let $\overline{A_{\mathrm{lg}}}$ be the completion of $A_{\mathrm{lg}}$. This algebra is both complete and semidagger by Proposition 3.8 . The canonical bounded homomorphism $A \rightarrow \widetilde{A_{\mathrm{lg}}}$ is the universal arrow from $A$ to a complete semi-dagger algebra, that is, any bounded homomorphism $A \rightarrow B$ for a complete semi-dagger algebra $B$ factors uniquely 
through it. This follows immediately from the universal properties of the linear growth bornology and the completion.

\section{Bornological torsion-freeness}

Let $M$ be a bornological $V$-module. The bounded linear map $\pi_{M}: M \rightarrow M$, $m \mapsto \pi \cdot m$, is defined in 2.10.

Definition 4.1. A bornological $V$-module $M$ is bornologically torsion-free if $\pi_{M}$ is a bornological embedding. Equivalently, $\pi \cdot m=0$ for $m \in M$ only happens for $m=0$ and any bounded subset of $M$ that is contained in $\pi \cdot M$ is of the form $S=\pi \cdot T$ for some $T \in \mathcal{B}_{M}$.

Bornological $F$-vector spaces are bornologically torsion-free because bornological isomorphisms are bornological embeddings. We are going to show that $M$ is bornologically torsion-free if and only if the canonical map $\iota_{M}: M \rightarrow F \otimes M$ defined in Lemma 2.12 is a bornological embedding. The proof uses the following easy permanence property:

Lemma 4.2. Let $M$ be a bornological $V$-module and let $N \subseteq M$ be a $V$-submodule with the subspace bornology. If $M$ is bornologically torsion-free, then so is $N$.

Proof. Let $j: N \rightarrow M$ be the inclusion map, which is a bornological embedding by assumption. Since $\pi_{M}$ is a bornological embedding, so is $\pi_{M} \circ j=j \circ \pi_{N}$. Since $j$ is a bornological embedding, this implies that $\pi_{N}$ is a bornological embedding. That is, $N$ is bornologically torsion-free.

Proposition 4.3. A bornological $V$-module $M$ is bornologically torsion-free if and only if the canonical map $\iota_{M}: M \rightarrow F \otimes M$ is a bornological embedding.

Proof. As a bornological $F$-vector space, $F \otimes M$ is bornologically torsionfree. Hence $M$ is bornologically torsion-free by Lemma 4.2 if $\iota_{M}$ is a bornological embedding. Conversely, assume that $M$ is bornologically torsion-free. The map $\iota_{M}$ is injective because $M$ is algebraically torsion-free. It remains to show that a subset $S$ of $M$ is bounded if $\iota_{M}(S) \subseteq F \otimes M$ is bounded. If $\iota_{M}(S)$ is bounded, then it is contained in $\pi^{-k} \cdot V \otimes T$ for some $k \in \mathbb{N}$ and some $T \in \mathcal{B}_{M}$. Equivalently, $\pi_{M}^{k}(S)=\pi^{k} \cdot S$ is bounded in $M$. Since $\pi_{M}$ is a bornological embedding, induction shows that $\pi_{M}^{k}: M \rightarrow M, m \mapsto \pi^{k} \cdot m$, is a bornological embedding as well. So the boundedness of $\pi_{M}^{k}(S)$ implies that $S$ is bounded.

Proposition 4.4. Let $M_{\mathrm{tf}}:=\iota_{M}(M) \subseteq F \otimes M$ equipped with the subspace bornology and the surjective bounded linear map $\iota_{M}: M \rightarrow M_{\mathrm{tf}}$. This is the universal arrow from $M$ to a bornologically torsion-free module, that is, any bounded linear map $f: M \rightarrow N$ into a bornologically torsion-free module $N$ factors uniquely through a bounded linear map $f^{\#:} M_{\mathrm{tf}} \rightarrow N$.

Proof. Since $F \otimes M$ is bornologically torsion-free as a bornological $F$-vector space, $M_{\mathrm{tf}}$ is bornologically torsion-free as well by Lemma 4.2 We prove the universality of the canonical map $\iota_{M}: M \rightarrow M_{\mathrm{tf}}$. Let $N$ be a bornologically torsionfree $V$-module and let $f: M \rightarrow N$ be a bornological $V$-module map. Then $N \hookrightarrow F \otimes N$ is a bornological embedding by Proposition 4.3 , and we may compose to get a bounded $V$-linear map $M \rightarrow F \otimes N$. By Lemma 2.12 , there is a unique bounded $F$-linear map $f^{\prime}: F \otimes M \rightarrow F \otimes N$ with $f^{\prime}\left(\iota_{M}(m)\right)=f(m)$ for all $m \in M$. Since 
$f^{\prime}\left(\iota_{M}(M)\right) \subseteq N, f^{\prime}$ maps the submodule $M_{\mathrm{tf}} \subseteq F \otimes M$ to the submodule $N \subseteq F \otimes N$. The restricted map $f^{\#}: M_{\mathrm{tf}} \rightarrow N$ is bounded because both submodules carry the subspace bornology. This is the required factorisation of $f$. It is unique because $\iota_{M}: M \rightarrow M_{\mathrm{tf}}$ is surjective.

We have seen that being bornologically torsion-free is hereditary for submodules. The obvious counterexample $\mathbb{F}=V / \pi V$ shows that it cannot be hereditary for quotients. Next we show that it is hereditary for extensions:

THEOREM 4.5. Let $M^{\prime} \stackrel{i}{\rightarrow} M \stackrel{q}{\rightarrow} M^{\prime \prime}$ be an extension of bornological $V$-modules. If $M^{\prime}$ and $M^{\prime \prime}$ are bornologically torsion-free, then so is $M$.

Proof. The exactness of the sequence $0 \rightarrow \operatorname{ker} \pi_{M^{\prime}} \rightarrow \operatorname{ker} \pi_{M} \rightarrow \operatorname{ker} \pi_{M^{\prime \prime}}$ shows that $\pi_{M}$ is injective. Let $S \in \mathcal{B}_{M}$ be contained in $\pi M$. We want a bounded subset $S^{\prime} \in \mathcal{B}_{M}$ with $\pi \cdot S^{\prime}=S$. We have $q(S) \subseteq q(\pi \cdot M) \subseteq \pi \cdot M^{\prime \prime}$, and $q(S) \in \mathcal{B}_{M^{\prime \prime}}$ because $q$ is bounded. Since $M^{\prime \prime}$ is bornologically torsion-free, there is $T^{\prime \prime} \in \mathcal{B}_{M^{\prime \prime}}$ with $\pi \cdot T^{\prime \prime}=q(S)$. Since $q$ is a bornological quotient map, there is $T \in \mathcal{B}_{M}$ with $q(T)=T^{\prime \prime}$. Thus $q(\pi \cdot T)=q(S)$. So for any $x \in S$ there is $y \in T$ with $q(\pi \cdot y)=q(x)$. Since $i=\operatorname{ker}(q)$, there is a unique $z \in M^{\prime}$ with $x-\pi y=i(z)$. Let $T^{\prime}$ be the set of these $z$. Since $x \in \pi \cdot M$ by assumption and $M^{\prime \prime}$ is torsion-free, we have $z \in \pi \cdot M^{\prime}$. So $T^{\prime} \subseteq \pi \cdot M^{\prime}$. And $T^{\prime}$ is bounded because $T^{\prime} \subseteq i^{-1}(S-\pi \cdot T)$ and $i$ is a bornological embedding, Since $M^{\prime}$ is bornologically torsion-free, there is a bounded subset $U^{\prime} \in \mathcal{B}_{M^{\prime}}$ with $\pi \cdot U^{\prime}=T^{\prime}$. Then $S \subseteq \pi \cdot T+i\left(\pi \cdot U^{\prime}\right)=\pi \cdot\left(T+i\left(U^{\prime}\right)\right)$.

Next we prove that bornological torsion-freeness is inherited by completions:

THEOREM 4.6. If $M$ is bornologically torsion-free, then so is its bornological completion $\vec{M}$.

The proof requires some preparation. We must look closely at the construction of completions of bornological $V$-modules.

Proposition 4.7 ([11, Proposition 2.15]). Let $M$ be a bornological $V$-module. A completion of $M$ exists and is constructed as follows. Write $M=\lim M_{i}$ as an inductive limit of the directed set of its bounded $V$-submodules. Let $\widehat{M}_{i}$ denote the $\pi$-adic completion of $M_{i}$. These form an inductive system as well, and $\mathbb{M} \cong$ $\left(\stackrel{\lim }{\longrightarrow} \widehat{M}_{i}\right) / \overline{\{0\}}$ is the separated quotient of their bornological inductive limit.

The completion functor commutes with colimits, that is, the completion of a colimit of a diagram of bornological $V$-modules is the separated quotient of the colimit of the diagram of completions.

Since taking quotients may create torsion, the information above is not yet precise enough to show that completions inherit bornological torsion-freeness. This requires some more work. First we write $M$ in a certain way as an inductive limit, using that it is bornologically torsion-free. For a bounded submodule $S$ in $M$, let

$$
\begin{aligned}
\pi^{-n} S & :=\left\{x \in M: \pi^{n} \cdot x \in S\right\} \subseteq M \\
M_{S} & :=\bigcup_{n \in \mathbb{N}} \pi^{-n} S \subseteq M .
\end{aligned}
$$

The gauge semi-norm of $S$ is defined by $\|x\|_{S}:=\inf \left\{\varepsilon^{n}: x \in \pi^{n} S\right\}$, where $\varepsilon=|\pi|$ (see [11. Example 2.4]). A subset is bounded for this semi-norm if and only if it is contained in $\pi^{-n} S$ for some $n \in \mathbb{N}$. Since $M$ is bornologically torsion-free, 
$\pi^{-n} S \in \mathcal{B}_{M}$ for $n \in \mathbb{N}$. So subsets that are bounded in the gauge semi-norm on $M_{S}$ are bounded in $M$. If $S \subseteq T$, then $M_{S} \subseteq M_{T}$ and the inclusion is contracting and hence bounded. The bornological inductive limit of this inductive system is naturally isomorphic to $M$ because any bounded subset of $M$ is bounded in $M_{S}$ for some bounded submodule $S \subseteq M$ (compare the proof of [11. Proposition 2.5]).

The bornological completion $\overline{M_{S}}$ of $M_{S}$ as a bornological $V$-module is canonically isomorphic to its Hausdorff completion as a semi-normed $V$-module. We call this a Banach $V$-module. Both completions are isomorphic to the increasing union of the $\pi$-adic completions $\widehat{\pi^{-n} S}$. If $S \subseteq T$, then $M_{S} \subseteq M_{T}$ and this inclusion is norm-contracting. So we get an induced contractive linear map $i_{T, S}: \overline{M_{S}} \rightarrow \overline{M_{T}}$. This map need not be injective any more (see 11, Example 2.15]). Hence the

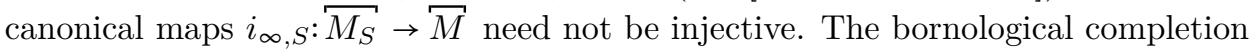
commutes with (separated) inductive limits by Proposition 4.7 So the completion of $M$ is isomorphic to the separated quotient of the colimit of the inductive system formed by the Banach $V$-modules $\overline{M_{S}}$ and the norm-contracting maps $i_{T, S}$ for $S \subseteq T$.

Lemma 4.8. The submodules

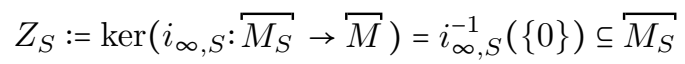

are norm-closed and satisfy $i_{T, S}^{-1}\left(Z_{T}\right)=Z_{S}$ if $S \subseteq T$. They are minimal with these properties in the sense that if $L_{S} \subseteq \widetilde{M_{S}}$ are norm-closed and satisfy $i_{T, S}^{-1}\left(L_{T}\right)=L_{S}$ for $S \subseteq T$, then $Z_{S} \subseteq L_{S}$ for all bounded $V$-submodules $S \subseteq M$.

Proof. The property $i_{T, S}^{-1}\left(Z_{T}\right)=Z_{S}$ is trivial. The map $i_{\infty, S}$ is bounded and hence preserves convergence of sequences. Since $\bar{M}$ is separated, the subset $\{0\} \subseteq \bar{M}$ is bornologically closed. Therefore, its preimage $Z_{S}$ in $\overline{M_{S}}$ is also closed. Let $\left(L_{S}\right)$ be any family of closed submodules with $i_{T, S}^{-1}\left(L_{T}\right)=L_{S}$. The quotient seminorm on $\widetilde{M_{S}} / L_{S}$ is again a norm because $L_{S}$ is closed. And $\overline{M_{S}} / L_{S}$ inherits completeness from $M_{S}$ by Theorem 2.3. If $S \subseteq T$, then $\iota_{T, S}$ induces an injective map $i_{T, S}^{\prime}: \overparen{M_{S}} / L_{S} \rightarrow \widetilde{M_{T}} / L_{T}$ because $L_{S}=i_{T, S}^{-1}\left(L_{T}\right)$. Hence the colimit of the inductive system $\left(\widetilde{M_{S}} / L_{S}, i_{T, S}^{\prime}\right)$ is like a directed union of subspaces, and each $\overparen{M_{S}} / L_{S}$ maps faithfully into it. Thus this colimit is separated. It is even complete because each $\widetilde{M_{S}} / L_{S}$ is complete. Hence the map from $M$ to this colimit induces a map on the completion $\vec{M}$. This implies $Z_{S} \subseteq L_{S}$.

Next we link $\overparen{M}$ to the $\pi$-adic completion $\widehat{M}:=\lim M / \pi^{j} M$. Equip the quotients $M / \pi^{j} M$ with the quotient bornology. Since $\pi^{j} \cdot\left(\overleftarrow{M /} \pi^{j}\right)=0$, any Cauchy sequence in $M / \pi^{j} M$ is eventually constant. So each $M / \pi^{j} M$ is complete. Hence the quotient map $M \rightarrow M / \pi^{j} M$ induces a bounded $V$-module homomorphism $M \rightarrow M / \pi^{j} M$. Putting them all together gives a map $\overparen{M} \rightarrow \widehat{M}$, which is bounded if we give $\widehat{M}$ the projective limit bornology.

Let $S \subseteq M$ be a bounded $V$-submodule and let $j \in \mathbb{N}$. We have defined the submodules $M_{S}$ so that $M_{S} \cap \pi^{j} M=\pi^{j} M_{S}$. That is, the map $M_{S} / \pi^{j} M_{S} \rightarrow M / \pi^{j} M$ is injective. Since $M_{S}$ is dense in its norm-completion $\widetilde{M_{S}}$, we have $\widetilde{M_{S}}=M_{S}+\pi^{j} \widehat{S}$ and hence $\overparen{M_{S}}=M_{S}+\pi^{j} \widetilde{M_{S}}$. Thus the inclusion $M_{S} \rightarrow \overparen{M_{S}}$ induces an isomorphism

$$
M_{S} / \pi^{j} M_{S} \cong \overparen{M_{S}} / \pi^{j} \widetilde{M_{S}} .
$$


Letting $j$ vary, we get an injective map $\widehat{M_{S}} \rightarrow \widehat{M}$ and an isomorphism between the $\pi$-adic completions of $M_{S}$ and $\overparen{M_{S}}$.

Proof of Theorem 4.6. For each bounded $V$-submodule $S \subseteq M$, define $L_{S}:=$ $\bigcap_{j \in \mathbb{N}} \pi^{j} \cdot \widetilde{M_{S}} \subseteq \overleftarrow{M_{S}}$. This is the kernel of the canonical map to the $\pi$-adic completion of $\overline{M_{S}}$. The completion $\overline{M_{S}}$ is torsion-free because it carries a norm. Hence $L_{S}$ is also the largest $F$-vector space contained in $M_{S}$. The subspace $L_{S}$ is closed because the maps $\overline{M_{S}} \rightarrow \widetilde{M_{S}} / \pi^{j} \widetilde{M_{S}}$ for $j \in \mathbb{N}$ are bounded and their target spaces are separable, even complete.

Let $S \subseteq T$. The maps $M_{S} / \pi^{j} M_{S} \rightarrow M_{T} / \pi^{j} M_{T}$ are injective for all $j \in \mathbb{N}$, and $\overline{M_{S}} / \pi^{j} \overline{M_{S}} \cong M_{S} / \pi^{j} M_{S}, \overline{M_{T}} / \pi^{j} \overline{M_{T}} \cong M_{T} / \pi^{j} M_{T}$. So $i_{T, S}$ induces an injective map $\overleftarrow{M_{S}} / \pi^{j} \overleftarrow{M_{S}} \rightarrow \overleftarrow{M_{T}} / \pi^{j} \overparen{M_{T}}$. This implies $i_{T, S}^{-1}\left(\pi^{j} \overparen{M_{T}}\right)=\pi^{j} \overleftarrow{M_{S}}$ for all $j \in \mathbb{N}$ and then $i_{T, S}^{-1}\left(L_{T}\right)=L_{S}$.

By Lemma 4.8, the kernel $Z_{S}=\operatorname{ker}\left(i_{\infty, S}\right)$ is contained in $L_{S}$ for all $S$. Since $\pi_{L_{S}}$ is a bornological isomorphism, the subsets $\pi \cdot Z_{S} \subseteq Z_{S}$ are also bornologically closed, and they satisfy $i_{T, S}^{-1}\left(\pi \cdot Z_{T}\right)=\pi \cdot i_{T, S}^{-1}\left(Z_{T}\right)=\pi \cdot Z_{S}$. Hence $Z_{S} \subseteq \pi \cdot Z_{S}$ for all $S$ by Lemma 4.8 Thus $Z_{S} \subseteq L_{S}$ is a $F$-vector subspace in $\widetilde{M_{S}}$. So the quotient $\overline{M_{S}} / Z_{S}$ is still bornologically torsion-free. And any element of $\overline{M_{S}} / Z_{S}$ that is divisible by $\pi^{j}$ lifts to an element in $\pi^{j} \cdot \overline{M_{S}}$.

Any bounded subset of $\bar{M}$ is contained in $i_{\infty, S}(\widehat{S})$ for some bounded $V$-submodule $S \subseteq M$, where we view $\widehat{S}$ as a subset of $\overleftarrow{M_{S}}$. Let $j \in \mathbb{N}$. To prove that $T$ is bornologically torsion-free, we must show that $\pi^{-j} i_{\infty, S}(\widehat{S})$ is bounded. Let $x \in \overparen{M}$ satisfy $\pi^{j} x \in i_{\infty, S}(\widehat{S})$. We claim that $x=i_{\infty, S}(y)$ for some $y \in \overline{M_{S}}$ with $\pi^{j} y \in \widehat{S}$. This implies that $\pi^{-j} \cdot i_{\infty, S}(\widehat{S})$ is bounded in $\overparen{M}$. It remains to prove the claim. There are a bounded $V$-submodule $T \subseteq M$ and $z \in \overline{M_{T}}$ with $x=i_{\infty, T}(z)$. We may replace $T$ by $T+S$ to arrange that $T \supseteq S$. Let $w \in \widehat{S}$ satisfy $\pi^{j} x=i_{\infty, S}(w)$. This is equivalent to $\pi^{j} z-i_{T, S}(w) \in \operatorname{ker} i_{\infty, T}=Z_{T}$. Since $Z_{T}$ is a $F$-vector space, there is $z_{0} \in Z_{T}$ with $\pi^{j} z-i_{T, S}(w)=\pi^{j} z_{0}$. Since $x=i_{\infty, T}\left(z-z_{0}\right)$, we may replace $z$ by $z-z_{0}$ to arrange that $\pi^{j} z=i_{T, S}(w)$. Since $i_{T, S}^{-1}\left(\pi^{j} \widetilde{M_{T}}\right)=\pi^{j} \widetilde{M_{S}}$, there is $y \in \overparen{M_{S}}$ with $\pi^{j} \cdot y=w$. Then $\pi^{j} z=\pi^{j} i_{T, S}(y)$. This implies $z=i_{T, S}(y)$ because $\overline{M_{T}}$ is torsion-free. This proves the claim.

Proposition 4.9. Let $M$ be a bornological $V$-module. Then $F \otimes \overparen{M} \cong \overparen{F \otimes M}$ with an isomorphism compatible with the canonical maps from $M$ to both spaces.

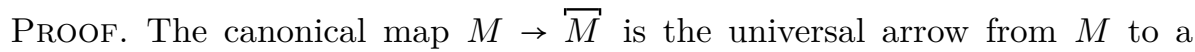
complete $V$-module. The canonical map $M \rightarrow F \otimes M$ is the universal arrow from $M$ to a bornological $F$-vector space by Lemma 2.12 Since $F \otimes \overparen{M}$ is again complete, the canonical map $M \rightarrow F \otimes T$ is the universal arrow from $M$ to a complete bornological $F$-vector space. The completion $\overparen{F \otimes M}$ is also a bornological $F$-vector space. The canonical map $M \rightarrow \overparen{F \otimes M}$ is another universal arrow from $M$ to a complete bornological $F$-vector space. Since the universal property determines its target uniquely up to canonical isomorphism, there is a unique isomorphism $F \otimes \overleftrightarrow{M} \cong \overparen{F \otimes M}$ that makes the following diagram commute:

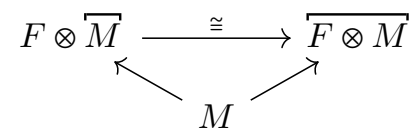


COROLlary 4.10. If $M$ is bornologically torsion-free, then the canonical map $\vec{M} \rightarrow \overrightarrow{F \otimes M}$ is a bornological embedding.

Proof. Use the isomorphism $F \otimes \overparen{M} \cong \overparen{F \otimes M}$ to replace the canonical map $\vec{M} \rightarrow \overrightarrow{F \otimes M}$ by the canonical map $\vec{M} \rightarrow F \otimes \vec{M}$. This is a bornological embedding if and only if $\overparen{M}$ is bornologically torsion-free by Proposition 4.3 . And this is true by Theorem 4.6 .

Finally, we show that being bornologically torsion-free is compatible with linear growth bornologies:

Proposition 4.11. If $A$ is a bornologically torsion-free $V$-algebra, then so is $A_{\mathrm{lg}}$.

Proof. Let $S \subseteq \pi \cdot A$ be bounded in $A_{\lg }$. Then there is $T \in \mathcal{B}_{A}$ with $S \subseteq T_{1}:=$ $\sum_{i=0}^{\infty} \pi^{i} T^{i+1}$ by Lemma 3.6 The subset $T_{2}:=\sum_{i=0}^{\infty} \pi^{i} T^{i+2}$ also has linear growth. And

$$
T_{1}=T+\sum_{i=1}^{\infty} \pi^{i} T^{i+1}=T+\sum_{i=0}^{\infty} \pi^{i+1} T^{i+2}=T+\pi T_{2} .
$$

Since $T$ is bounded in $A$ and $A$ is bornologically torsion-free, $\pi^{-1} \cdot T:=\{x \in A: \pi \cdot x \in T\}$ is also bounded. We have $\pi^{-1} S \subseteq \pi^{-1} T_{1} \subseteq \pi^{-1} \cdot T+T_{2}$. This is bounded in $A_{\lg }$.

The following proposition answers a question by Guillermo Cortiñas:

Proposition 4.12. Let $M$ and $N$ be bornological $V$-modules. If $M$ and $N$ are bornologically torsion-free, then so is $M \otimes N$ with the tensor product bornology.

Proof. Since $M$ and $N$ are torsion-free, so is $M \otimes N$, that is, multiplication by $\pi$ on $M \otimes N$ is injective. Let $U \subseteq M \otimes N$ be a subset such that $\pi U$ is bounded. We must show that $U$ is bounded. By the definition of the tensor product bornology, there are bounded $V$-submodules $S \subseteq M, T \subseteq N$ such that $\pi \cdot U \subseteq S \otimes T$. Define

$$
\pi^{-1} S:=\{x \in M: \pi x \in S\}, \quad \pi^{-1} T:=\{y \in N: \pi y \in T\} .
$$

These subsets are bounded because $\pi \cdot\left(\pi^{-1} S\right) \subseteq S$ and $\pi \cdot\left(\pi^{-1} T\right) \subseteq T$ and $M$ and $N$ are bornologically torsion-free. We claim that $U \subseteq \pi^{-1} S \otimes \pi^{-1} T$. This shows that $U$ is bounded.

Let $u \in U$. We may write $u=\sum_{j=1}^{N} x_{j} \otimes y_{j}$ with $x_{j} \in M, y_{j} \in N$. Since $\pi \cdot u \in S \otimes T$, we may write $\pi u=\sum_{k=1}^{M} z_{k} \otimes w_{k}$ with $z_{k} \in S, w_{k} \in T$. Let $A \subseteq M$ and $B \subseteq N$ be the $V$-submodules generated by the elements $x_{j}, z_{k}$ and $y_{j}, w_{k}$, respectively. These submodules are finitely generated and torsion-free, hence free. And the canonical map $A \otimes B \rightarrow M \otimes N$ is injective. The submodules $A \cap S$ and $B \cap T$ are also free. Any $V$-module homomorphism between finitely generated free $V$-modules may be brought into diagonal form with entries in $\left\{\pi^{\mathbb{N}}\right\} \cup\{0\}$ along the diagonal by choosing appropriate bases in the $V$-modules. Therefore, there are $V$-module bases $a_{1}, \ldots, a_{n}$ and $b_{1}, \ldots, b_{m}$ of $A$ and $B$, respectively, and $1 \leq n^{\prime} \leq n, 1 \leq m^{\prime} \leq m$, $0 \geq \alpha_{1} \geq \alpha_{2} \geq \cdots \geq \alpha_{n^{\prime}}, 0 \geq \beta_{1} \geq \beta_{2} \geq \cdots \geq \beta_{m^{\prime}}$, such that $\pi^{\alpha_{i}} \cdot a_{i}$ and $\pi^{\beta_{j}} \cdot b_{j}$ for $1 \leq i \leq n^{\prime}$ and $1 \leq j \leq m^{\prime}$ are $V$-module bases of $A \cap S$ and $B \cap T$, respectively. We may write $u \in A \otimes B$ uniquely in this basis as $u=\sum_{i, j} u_{i, j} a_{i} \otimes b_{j}$ with $u_{i, j} \in V$. By assumption, $\pi \cdot u=\sum_{k=1}^{M} y_{k} \otimes w_{k} \in(S \cap A) \otimes(T \cap B)$. By construction, the elements $\pi^{\alpha_{i}+\beta_{j}} a_{i} \otimes b_{j}$ form a $V$-module basis of $(S \cap A) \otimes(T \cap B)$. Since the coefficients of $\pi u$ in the basis $a_{i} \otimes b_{j}$ of $A \otimes B$ are unique, it follows that $u_{i, j}=0$ if $i>n^{\prime}$ or $j>m^{\prime}$, and $\pi u_{i, j} \in \pi^{\alpha_{i}+\beta_{j}} V$ for $1 \leq i \leq n^{\prime}$ and $1 \leq j \leq m^{\prime}$. Hence $u$ is a $V$-linear combination 
of $\pi^{\left(\alpha_{i}-1\right)_{+}} a_{i} \otimes \pi^{\left(\beta_{j}-1\right)_{+}} b_{j}$ for $1 \leq i \leq n^{\prime}, 1 \leq j \leq m^{\prime}$, where $n_{+}:=\max \{n, 0\}$. Since $\pi^{\left(\alpha_{i}-1\right)_{+}} a_{i} \in \pi^{-1} S, \pi^{\left(\alpha_{j}-1\right)_{+}} b_{j} \in \pi^{-1} T$, this implies $u \in \pi^{-1} S \otimes \pi^{-1} T$. Since $u \in U$ was arbitrary, we get $U \subseteq \pi^{-1} S \otimes \pi^{-1} T$.

Theorem 4.6 and Proposition 4.12 imply that bornological torsion-freeness for complete bornological $V$-modules is hereditary for completed tensor products.

\section{Dagger algebras}

Definition 5.1. A dagger algebra is a complete, bornologically torsion-free, semi-dagger algebra.

Theorem 5.2. Let $A \stackrel{i}{\rightarrow} B \stackrel{p}{\rightarrow} C$ be an extension of bornological $V$-algebras. If $A$ and $C$ are dagger algebras, so is $B$.

Proof. All three properties defining dagger algebras are hereditary for extensions by Theorems 2.3 (because $C$ is torsion-free), 3.7 and 4.5

We have already seen that there are universal arrows $A \rightarrow A_{\mathrm{tf}} \subseteq F \otimes A, A \rightarrow A_{\mathrm{lg}}$, $A \rightarrow \bar{A}$ from a bornological algebra $A$ to a bornologically torsion-free algebra, to a semi-dagger algebra, and to a complete bornological algebra, respectively. We now combine them to a universal arrow to a dagger algebra:

Theorem 5.3. Let $A$ be a bornological algebra. Then the canonical map from $A$ to $A^{\dagger}:=\overparen{\left(A_{\mathrm{tf}}\right)_{\mathrm{gg}}}$ is the universal arrow from $A$ to a dagger algebra. That is, any bounded algebra homomorphism from $A$ to a dagger algebra factors uniquely through $A^{\dagger}$. If $A$ is already bornologically torsion-free, then $A^{\dagger} \cong \overparen{A_{\mathrm{lg}}}$.

Proof. The bornological algebra $A^{\dagger}$ is complete by construction. It is semidagger by Proposition 3.8 And it is bornologically torsion-free by Proposition 4.11 and Theorem 4.6 So it is a dagger algebra. Let $B$ be a dagger algebra. A bounded homomorphism $A \rightarrow B$ factors uniquely through a bounded homomorphism $A_{\mathrm{tf}} \rightarrow B$ by Proposition 4.4 because $B$ is bornologically torsion-free. This factors uniquely through a bounded homomorphism $\left(A_{\mathrm{tf}}\right)_{\mathrm{lg}} \rightarrow B$ because $B$ is semi-dagger. And this factors uniquely through a bounded homomorphism $\overline{\left(A_{\mathrm{tf}}\right)_{\mathrm{lg}}} \rightarrow B$ because $B$ is complete. So $A^{\dagger}$ has the asserted universal property. If $A$ is bornologically torsion-free, then $A \cong A_{\mathrm{tf}}$ and hence $A^{\dagger} \cong \widetilde{A_{\mathrm{lg}}}$.

Definition 5.4. We call $A^{\dagger}$ the dagger completion of the bornological $V$-algebra $A$.

\section{Dagger completions of monoid algebras}

As a simple illustration, we describe the dagger completions of monoid algebras. The monoid algebra of $\mathbb{N}^{j}$ is the algebra of polynomials in $j$ variables, and its dagger completion is the Monsky-Washnitzer algebra of overconvergent power series equipped with a canonical bornology (see 11]). The case of general monoids is similar.

The monoid algebra $V[S]$ of $S$ over $V$ is defined by its universal property: if $B$ is a unital $V$-algebra, then there is a natural bijection between algebra homomorphisms $V[S] \rightarrow B$ and monoid homomorphisms $S \rightarrow(B, \cdot)$ into the multiplicative monoid 
of $B$. More concretely, $V[S]$ is the free $V$-module with basis $S$ or, equivalently, the $V$-module of formal linear combinations of the form

$$
\sum_{s \in S} x_{s} \delta_{s}, \quad x_{s} \in V, s \in S
$$

with $x_{s}=0$ for all but finitely many $s$, and equipped with the multiplication

$$
\sum_{s \in S} x_{s} \delta_{s} * \sum_{t \in S} y_{t} \delta_{t}=\sum_{s, t \in S} x_{s} y_{t} \delta_{s \cdot t} .
$$

We give $V[S]$ the fine bornology. Then it has an analogous universal property in the category of bornological $V$-algebras. So the dagger completion $V[S]^{\dagger}$ is a dagger algebra with the property that bounded algebra homomorphisms $V[S]^{\dagger} \rightarrow B$ for a dagger algebra $B$ are in natural bijection with monoid homomorphisms $S \rightarrow(B, \cdot)$.

Assume first that $S$ has a finite generating set $F$. Let $F^{n} \subseteq S$ be the set of all words $s_{1} \cdots s_{k}$ with $s_{1}, \ldots, s_{k} \in F$ and $k \leq n$. This gives an increasing filtration on $S$ with $F^{0}=\{1\}$ and $S=\cup_{n=0}^{\infty} F^{n}$. For $s \in S$, we define $\ell(s) \in \mathbb{N}$ as the smallest $n$ with $s \in F^{n}$. This is the word length generated by $F$. Let $V\left[F^{n}\right] \subseteq V[S]$ be the free $V$-submodule of $V[S]$ spanned by $F^{n}$. Any finitely generated $V$-submodule of $V[S]$ is contained in $V\left[F^{n}\right]$ for some $n \in \mathbb{N}$. By Lemma 3.6, a subset of $V[S]$ has linear growth if and only if it is contained in $M_{n}:=\sum_{j=0}^{\infty} \pi^{j}\left(V\left[F^{n}\right]\right)^{j+1}$ for some $n \in \mathbb{N}_{\geq 1}$. That is,

$$
M_{\mathrm{lg}}=\underset{\lim }{\longrightarrow} M_{n} .
$$

Recall the valuation $\nu: V \rightarrow \mathbb{N} \cup\{\infty\}$ defined by

$$
\nu(x):=\sup \left\{n \in \mathbb{N}: x \in \pi^{n} V\right\} .
$$

By definition, the submodule $M_{n}$ consists of all finite sums of terms $x_{s} \delta_{s}$ with $x_{s} \in \pi^{j} \cdot V$ and $\ell(s) \leq n(j+1)$ for some $j \in \mathbb{N}$ or, equivalently, $\ell(s) / n \leq j+1 \leq \nu\left(x_{s}\right)+1$. That is, $M_{n}$ contains a finite sum $\sum_{s \in S} x_{s} \delta_{s}$ with $x_{s} \in V$ and $x_{s}=0$ for all but finitely many $s \in S$ if and only if $\nu\left(x_{s}\right)+1 \geq \ell(s) / n$ for all $s \in S$. The $\pi$-adic completion $\widehat{M_{n}}$ of $M_{n}$ is the set of all formal power series $\sum_{s \in S} x_{s} \delta_{s}$ such that $x_{s} \in V$, $\nu\left(x_{s}\right)+1 \geq \ell(s) / n$ for all $s \in S$ and $\lim _{\ell(s) \rightarrow \infty} \nu\left(x_{s}\right)+1-\ell(s) / n=\infty$. This implies $x_{s} \rightarrow 0$ in the $\pi$-adic norm, so that $\widehat{M_{n}} \subseteq \widehat{V[S]}$. So the extension $\widehat{M_{n}} \rightarrow \widehat{M_{n+1}}$ of the inclusion map $M_{n} \rightarrow M_{n+1}$ remains injective. Therefore, $\underset{\longrightarrow}{\longrightarrow} \widehat{M_{n}}$ is separated, and it is contained in $\widehat{V[S]}$. Proposition 4.7 implies

$$
V[S]^{\dagger}=\stackrel{\lim }{\longrightarrow} \widehat{M_{n}} .
$$

Elements of $\overline{V[S]}$ are formal series $\sum_{s \in S} x_{s} \delta_{s}$ with $x_{s} \in V$ for all $s \in S$ and $\lim \left|x_{s}\right|=0$. We have seen above that such a formal series belongs to $\widehat{M_{n}}$ if and only if $\nu\left(x_{s}\right)+1 \geq \ell(s) / n$ for all $s \in S$ and $\lim _{\ell(s) \rightarrow \infty} \nu\left(x_{s}\right)+1-\ell(s) / n=\infty$. If $0<1 / n<c$, then $\nu\left(x_{s}\right)+1 \geq c \ell(s)$ implies $\nu\left(x_{s}\right)+1 \geq \ell(s) / n$ and $\lim _{\ell(s) \rightarrow \infty} \nu\left(x_{s}\right)+1-\ell(s) / n=\infty$. Thus all $\sum_{s \in S} x_{s} \delta_{s} \in \widehat{V[S]}$ with $\nu\left(x_{s}\right)+1 \geq c \ell(s)$ belong to $\widehat{M_{n}}$. Conversely, all elements of $\widehat{M_{n}}$ satisfy this for $c=1 / n$. Letting $c$ and $n$ vary, we see that $V[S]^{\dagger}$ is the set of all $\sum_{s \in S} x_{s} \delta_{s}$ in $\overline{V[S]}$ for which there is $c>0$ with

$$
\nu\left(x_{s}\right)+1 \geq c \ell(s) \quad \text { for all } s \in S,
$$

and that a subset of $V[S]^{\dagger}$ is bounded if and only if all its elements satisfy 6.1 for the same $c>0$. The growth condition 6.1 does not depend on the word length function $\ell$ because the word length functions for two different generating sets of $S$ are related by linear inequalities $\ell \leq a \ell^{\prime}$ and $\ell^{\prime} \leq a \ell$ for some $a>0$. 
Now we drop the assumption that $S$ be finitely generated. Then we may write $S$ as the increasing union of its finitely generated submonoids. By the universal property, the monoid algebra of $S$ with the fine bornology is a similar inductive limit in the category of bornological $V$-algebras, and its dagger algebra is the inductive limit in the category of dagger algebras. Since $V\left[S^{\prime}\right]^{\dagger} \subseteq \overline{V\left[S^{\prime}\right]} \subseteq \widehat{V[S]}$ for any finitely generated $S^{\prime} \subseteq S$, we may identify this inductive limit with a subalgebra of $\overline{V[S]}$ as well, namely, the union of $V\left[S^{\prime}\right]^{\dagger}$ over all finitely generated submonoids $S^{\prime} \subseteq S$. That is, $V[S]^{\dagger}$ is the set of elements of $\overline{V[S]}$ that are supported in some finitely generated submonoid $S^{\prime} \subseteq S$ and that satisfy 6.1 for some length function on $S^{\prime}$.

We may also twist the monoid algebra. Let $V^{\times}=\{x \in V:|x|=1\}$ and let $c: S \times S \rightarrow V^{\times}$be a normalised 2-cocycle, that is,

$$
c(r, s \cdot t) \cdot c(s, t)=c(r \cdot s, t) \cdot c(r, s), \quad c(s, 1)=c(1, s)=1
$$

for all $r, s, t \in S$. The $c$-twisted monoid algebra of $S, V[S, c]$, is the $V$-module $V[S]$ with the twisted multiplication

$$
\sum_{s \in S} x_{s} \delta_{s} * \sum_{t \in S} y_{t} \delta_{t}=\sum_{s, t \in S} x_{s} y_{t} c(s, t) \cdot \delta_{s \cdot t} .
$$

The condition $(6.2)$ is exactly what is needed to make this associative and unital with unit $\delta_{1}$. Since we assume $c$ to have values in $V^{\times}$, the twist does not change the linear growth bornology. Therefore, the dagger completion $V[S, c]^{\dagger}$ consists of all infinite sums $\sum_{s \in S} x_{s} \delta_{s}$ that are supported in a finitely generated submonoid of $S$ and satisfy the growth condition (6.1), and a subset is bounded if and only if all its elements satisfy these two conditions uniformly. Only the multiplication changes and is now given by 6.3 .

Example 6.4. Let $S=\left(\mathbb{Z}^{2},+\right)$ with the unit element 0. Define $c\left(\left(s_{1}, s_{2}\right),\left(t_{1}, t_{2}\right)\right):=$ $\lambda^{s_{2} \cdot t_{1}}$ for some $\lambda \in V^{\times}$. This satisfies 6.2 . The resulting twisted convolution algebra is an analogue of a noncommutative torus over $V$. Indeed, let $U_{1}:=\delta_{(1,0)}$ and $U_{2}:=\delta_{(0,1)}$ as elements of $V\left[\mathbb{Z}^{2}, c\right]$. Then $\delta_{(-1,0)}=U_{1}^{-1}$ and $\delta_{(0,-1)}=U_{2}^{-1}$ are inverse to them, and $\delta_{\left(s_{1}, s_{2}\right)}=U_{1}^{s_{1}} \cdot U_{2}^{s_{2}}$. So $U_{1}, U_{2}$ generate $V\left[\mathbb{Z}^{2}, c\right]$ as a $V$-algebra. They satisfy the commutation relation

$$
U_{2} \cdot U_{1}=\lambda \cdot U_{1} \cdot U_{2}
$$

And this already dictates the multiplication table in $V\left[\mathbb{Z}^{2}, c\right]$. The dagger completion $V\left[\mathbb{Z}^{2}, c\right]^{\dagger}$ is isomorphic as a bornological $V$-module to the Monsky-Washnitzer completion of the Laurent polynomial algebra $V\left[U_{1}^{ \pm 1}, U_{2}^{ \pm 1}\right]$, equipped with a twisted multiplication satisfying 6.5).

\section{Dagger completions of crossed products}

Let $A$ be a unital, bornological $V$-algebra, let $S$ be a finitely generated monoid and let $\alpha: S \rightarrow \operatorname{End}(A)$ be an action of $S$ on $A$ by bounded algebra homomorphisms. The crossed product $A \rtimes_{\alpha} S$ is defined as follows. Its underlying bornological $V$-module is $A \rtimes_{\alpha} S=\oplus_{s \in S} A$ with the direct sum bornology. So elements of $A \rtimes_{\alpha} S$ are formal linear combinations $\sum_{s \in S} a_{s} \delta_{s}$ with $a_{s} \in A$ and $a_{s}=0$ for all but finitely many $s \in S$. The multiplication on $A \rtimes_{\alpha} S$ is defined by

$$
\left(\sum_{s \in S} a_{s} \delta_{s}\right) \cdot\left(\sum_{t \in S} b_{t} \delta_{t}\right):=\sum_{s, t \in S} a_{s} \alpha_{s}\left(b_{t}\right) \delta_{s t}
$$

This makes $A \rtimes_{\alpha} S$ a bornological $V$-algebra. What is its dagger completion? 
It follows easily from the universal property that defines $A \subseteq A \rtimes_{\alpha} S$ that

$$
\left(A \rtimes_{\alpha} S\right)^{\dagger} \cong\left(A^{\dagger} \rtimes_{\alpha^{\dagger}} S\right)^{\dagger} \text {; }
$$

here $\alpha^{\dagger}$ is the canonical extension of $\alpha$ to the dagger completion $A^{\dagger}$, which exists because the latter is functorial for bounded algebra homomorphisms. Therefore, it is no loss of generality to assume that $A$ is already a dagger algebra. It is easy to show that $(A \rtimes S)^{\dagger}$ is the inductive limit of the dagger completions $\left(A \rtimes S^{\prime}\right)^{\dagger}$, where $S^{\prime}$ runs through the directed set of finitely generated submonoids of $S$. Hence we may also assume that $S$ is finitely generated to simplify. First we consider the following special case:

Definition 7.1. The action $\alpha: S \rightarrow \operatorname{End}(A)$ is called uniformly bounded if any $U \in \mathcal{B}_{A}$ is contained in an $\alpha$-invariant $T \in \mathcal{B}_{A} ; \alpha$-invariance means $\alpha_{s}(T)=T$ for all $s \in S$.

If $T$ is $\alpha$-invariant, so is the $V$-module generated by $T$. Therefore, $\alpha$ is uniformly bounded if and only if any bounded subset of $A$ is contained in a bounded, $\alpha$-invariant $V$-submodule. If $A$ is complete, then the image of $\widehat{T}$ in $A$ is also $\alpha$-invariant because the maps $\alpha_{s}$ are bornological isomorphisms. Hence we may assume in this case that $T$ in Definition 7.1 is a bounded, $\alpha$-invariant $\pi$-adically complete $V$-submodule.

Proposition 7.2. Let A carry a uniformly bounded action $\alpha$ of $S$. Then the induced actions on $\vec{A}, A_{\mathrm{tf}}$, and $A_{\mathrm{lg}}$ are uniformly bounded as well. Hence so is the induced action on $A^{\dagger}$.

Proof. If $\alpha$ is uniformly bounded, then $A$ is the bornological inductive limit of its $\alpha$-invariant bounded $V$-submodules. The action of $\alpha$ restricts to any such submodule $T$ and then extends canonically to its $\pi$-adic completion $\widehat{T}$. Then the image of $\widehat{T}$ in $\bar{A}$ is $S$-invariant as well. This gives enough $S$-invariant bounded $V$-submodules in $\vec{A}$. So the induced action on $\vec{A}$ is uniformly bounded.

If the action $\alpha$ on $A$ is uniformly bounded, then so is the action $\operatorname{id}_{B} \otimes \alpha$ on $B \otimes A$ for any bornological algebra $B$. In particular, the induced action on $F \otimes A$ is uniformly bounded. Since the canonical map $A \rightarrow F \otimes A$ is $S$-equivariant, the image $A_{\text {tf }}$ of $A$ in $F \otimes A$ is $S$-invariant. The restriction of the uniformly bounded action of $S$ on $F \otimes A$ to this invariant subalgebra inherits uniform boundedness. So the induced action on $A_{\mathrm{tf}}$ is uniformly bounded.

Any subset of linear growth in $A$ is contained in $\sum_{j=0}^{\infty} \pi^{j} T^{j+1}$ for a bounded $V$-submodule $T$. Since $\alpha$ is uniformly bounded, $T$ is contained in an $\alpha$-invariant bounded $V$-submodule $U$. Then $\sum_{j=0}^{\infty} \pi^{j} U^{j+1} \supseteq \sum_{j=0}^{\infty} \pi^{j} T^{j+1}$ is $\alpha$-invariant and has linear growth. So $\alpha$ remains uniformly bounded for the linear growth bornology.

The uniform boundedness of the induced action on the dagger completion $A^{\dagger}$ follows from the inheritance properties above and Theorem 5.3 .

ExAmple 7.3. Let $S$ be a finite monoid. Any bounded action of $S$ by bornological algebra endomorphisms is uniformly bounded because we may take $T=\sum_{s \in S} \alpha_{s}(U)$ in Definition 7.1

EXAMPLE 7.4. We describe a uniformly bounded action of $\mathbb{Z}$ on the polynomial algebra $A:=V\left[x_{1}, \ldots, x_{n}\right]$ with the fine bornology. So a subset of $A$ is bounded if and only if it is contained in $\left(V+V x_{1}+\cdots+V x_{n}\right)^{k}$ for some $k \in \mathbb{N}_{\geq 1}$. Let $a \in \operatorname{GL}_{n}(V) \subseteq \operatorname{End}\left(V^{n}\right)$ and $b \in V^{n}$. Then

$$
\alpha_{1}: V\left[x_{1}, \ldots, x_{n}\right] \rightarrow V\left[x_{1}, \ldots, x_{n}\right], \quad\left(\alpha_{1} f\right)(x):=f(a x+b),
$$


is an algebra automorphism $\alpha_{1}$ of $A$ with inverse $\left(\alpha_{1}^{-1} f\right)(x):=f\left(a^{-1}(x-b)\right)$. This generates an action of the group $\mathbb{Z}$ by $\alpha_{n}:=\alpha_{1}^{n}$ for $n \in \mathbb{Z}$. If a polynomial $f$ has degree at most $m$, then the same is true for $\alpha_{1} f$ and $\alpha_{-1} f$, and hence for $\alpha_{n} f$ for all $n \in \mathbb{Z}$. That is, the $V$-submodules $\left(V+V x_{1}+\cdots+V x_{n}\right)^{k}$ in $A$ for $k \in \mathbb{N}$ are $\alpha$-invariant. So the action $\alpha$ on $A$ is uniformly bounded. Proposition 7.2 implies that the induced action on $V\left[x_{1}, \ldots, x_{n}\right]^{\dagger}$ is uniformly bounded as well.

Proposition 7.5. Let $S$ be a finitely generated monoid with word length function $\ell$. Let $A$ be a dagger algebra and let $\alpha: S \rightarrow \operatorname{End}(A)$ be a uniformly bounded action by algebra endomorphisms. Then $(A \rtimes S)^{\dagger} \subseteq \prod_{s \in S} A$. A formal series $\sum_{s \in S} a_{s} \delta_{s}$ with $a_{s} \in A$ for all $s \in S$ belongs to $(A \rtimes S)^{\dagger}$ if and only if there are $\varepsilon>0$ and $T \in \mathcal{B}_{A}$ with $a_{s} \in \pi^{\lfloor\varepsilon \ell(s)\rfloor} T$ for all $s \in S$, and a set of formal series is bounded in $(A \rtimes S)^{\dagger}$ if and only if $\varepsilon>0$ and $T \in \mathcal{B}_{A}$ for its elements may be chosen uniformly.

Proof. We first describe the linear growth bornology on $A \rtimes S$. Let $\mathcal{B}^{\prime}$ be the set of all subsets $U \subseteq A \rtimes S$ for which there are $T \in \mathcal{B}_{A}$ and $\varepsilon>0$ such that any element of $U$ is of the form $\sum_{s \in S} a_{s} \delta_{s}$ with $a_{s} \in \pi^{\lfloor\varepsilon \ell(s)\rfloor} T$ for all $s \in S$. We claim that $\mathcal{B}^{\prime}$ is the linear growth bornology on $A \rtimes S$. The inclusion $V[S] \subseteq A \rtimes S$ induces a bounded algebra homomorphism $V[S]_{\lg } \rightarrow(A \rtimes S)_{\lg }$. We have already described the linear growth bornology on $V[S]$ in Section 6 This implies easily that all subsets in $\mathcal{B}^{\prime}$ have linear growth: write $\pi^{\lfloor\varepsilon \ell(s)\rfloor} a_{s}^{\prime} \delta_{s}=a_{s}^{\prime} \cdot \pi^{\lfloor\varepsilon \ell(s)\rfloor} \delta_{s}$. We claim, conversely, that any subset of $A \rtimes S$ of linear growth is contained in $\mathcal{B}^{\prime}$. All bounded subsets of $A \rtimes S$ are contained in $\mathcal{B}^{\prime}$. It is routine to show that $\mathcal{B}^{\prime}$ is a $V$-algebra bornology. We only prove that the bornology $\mathcal{B}^{\prime}$ has linear growth. Since $\alpha$ is uniformly bounded, any $T \in \mathcal{B}_{A}$ is contained in a bounded, $\alpha$-invariant $V$-submodule $T_{2}$. Then $T_{3}:=\sum_{j=0}^{\infty} \pi^{j} T_{2}^{j+1}$ is a bounded, $\alpha$-invariant $V$-submodule with $\pi \cdot T_{3}^{2} \subseteq T_{3}$ and $T \subseteq T_{3}$ (see [11. Equation (5)]). If $a_{s} \in \pi^{\lfloor\varepsilon \ell(s)\rfloor} T_{3}$ and $a_{t} \in \pi^{\lfloor\varepsilon \ell(t)\rfloor} T_{3}$, then

$$
\pi^{2} \cdot a_{s} \cdot \alpha_{t} \in \pi^{2+\lfloor\varepsilon \ell(s)\rfloor+\lfloor\varepsilon \ell(t)\rfloor} T_{3}^{2} \subseteq \pi^{\lfloor\varepsilon \ell(s \cdot t)\rfloor} \pi T_{3}^{2} \subseteq \pi^{\lfloor\varepsilon \ell(s \cdot t)\rfloor} T_{3}
$$

because $1+\lfloor\varepsilon \ell(s)\rfloor+\lfloor\varepsilon \ell(t)\rfloor \geq\lfloor\varepsilon \ell(s \cdot t)\rfloor$. This implies

$$
\pi^{2} \cdot \sum_{s \in S} \pi^{\lfloor\varepsilon \ell(s)\rfloor} T_{3} \delta_{s} * \sum_{t \in S} \pi^{\lfloor\varepsilon \ell(t)\rfloor} T_{3} \delta_{t} \subseteq \sum_{s, t \in S} \pi^{\lfloor\varepsilon \ell(s \cdot t)\rfloor} T_{3} \delta_{s t} \subseteq \sum_{s \in S} \pi^{\lfloor\varepsilon \ell(s)\rfloor} T_{3} \delta_{s} .
$$

So any subset in $\mathcal{B}^{\prime}$ is contained in $U \in \mathcal{B}^{\prime}$ with $\pi^{2} \cdot U^{2} \subseteq U$. By induction, this implies $\left(\pi^{2} U\right)^{k} \cdot U \subseteq U$ for all $k \in \mathbb{N}$. Hence $\sum_{j=0}^{\infty} \pi^{2 k} U^{k+1}$ is in $\mathcal{B}^{\prime}$. Now Lemma 3.6 shows that the bornology $\mathcal{B}^{\prime}$ is semi-dagger. This proves the claim that $\mathcal{B}^{\prime}$ is the linear growth bornology on $A \rtimes S$.

Since $A$ as a dagger algebra is bornologically torsion-free, so is $A \rtimes S$. So $(A \rtimes S)^{\dagger}$ is the completion of $(A \rtimes S)_{\lg }=\left(A \rtimes S, \mathcal{B}^{\prime}\right)$. It is routine to identify this completion with the bornological $V$-module described in the statement.

Propositions 7.2 and 7.5 describe the dagger completion of $A \rtimes S$ for a uniformly bounded action of $S$ on $A$ even if $A$ is not a dagger algebra. Namely, the universal properties of the crossed product and the dagger completion imply

$$
(A \rtimes S)^{\dagger} \cong\left(A^{\dagger} \rtimes S\right)^{\dagger} .
$$

EXAMPLE 7.7. Let $\alpha$ be the uniformly bounded action of $\mathbb{Z}$ on $V\left[x_{1}, \ldots, x_{k}\right]$ from Example 7.4 The induced action $\alpha^{\dagger}$ on $V\left[x_{1}, \ldots, x_{k}\right]^{\dagger}$ is also uniformly bounded by Proposition 7.2 And (7.6) implies

$$
\left(V\left[x_{1}, \ldots, x_{k}\right] \rtimes_{\alpha} \mathbb{Z}\right)^{\dagger} \cong\left(V\left[x_{1}, \ldots, x_{k}\right]^{\dagger} \rtimes_{\alpha^{\dagger}} \mathbb{Z}\right)^{\dagger} .
$$


The latter is described in Proposition 7.5 Namely, $\left(V\left[x_{1}, \ldots, x_{k}\right]^{\dagger} \rtimes_{\alpha^{\dagger}} \mathbb{Z}\right)^{\dagger}$ consists of those formal series $\sum_{n \in \mathbb{Z}} a_{n} \delta_{n}$ with $a_{n} \in V\left[x_{1}, \ldots, x_{k}\right]^{\dagger}$ for which there are $\varepsilon>0$ and a bounded $V$-submodule $T$ in $V\left[x_{1}, \ldots, x_{k}\right]^{\dagger}$ such that $a_{n} \in \pi^{\lfloor\varepsilon|n|\rfloor} T$ for all $n \in \mathbb{Z}$; notice that $|n|$ is indeed a length function on $\mathbb{Z}$. And a subset is bounded if some pair $\varepsilon, T$ works for all its elements.

We combine this with the description of bounded subsets of $V\left[x_{1}, \ldots, x_{k}\right]^{\dagger}$ in Section 6 there is some $\delta>0$ so that a formal power series $\sum_{m \in \mathbb{N}^{k}} b_{m} x^{m}$ belongs to $T$ if and only if $b_{m} \in \pi^{[\delta|m|\rfloor} V$ for all $m \in \mathbb{N}^{k}$. Here we use the length function $\left|\left(m_{1}, \ldots, m_{k}\right)\right|=\sum_{j=1}^{k} m_{j}$. We may merge the parameters $\varepsilon, \delta>0$ above, taking their minimum. So $\left(V\left[x_{1}, \ldots, x_{k}\right] \rtimes \mathbb{Z}\right)^{\dagger}$ consists of the formal series $\sum_{n \in \mathbb{Z}, m \in \mathbb{N}^{k}} a_{n, m} x^{m} \delta_{n}$ with $a_{n, m} \in \pi^{\lfloor\varepsilon(|n|+|m|)\rfloor} V$ or, equivalently, $\nu\left(a_{n, m}\right)+1>\varepsilon(|n|+|m|)$ for all $n \in \mathbb{Z}$, $m \in \mathbb{N}^{k}$.

If the action of $S$ on $A$ is not uniformly bounded, then the linear growth bornology on $A \rtimes S$ becomes much more complicated. It seems unclear whether the description below helps much in practice. Let $F \subseteq S$ be a finite generating subset containing 1. Any bounded subset of $A \rtimes S$ is contained in $\left(\sum_{s \in F} T \cdot \delta_{s}\right)^{N}$ for some $N \in \mathbb{N}$ and some $T \in \mathcal{B}_{A}$ with $1 \in T$. Therefore, a subset of $A \rtimes S$ has linear growth if and only if it is contained in the $V$-submodule generated by

$$
\bigcup_{n=1}^{\infty} \pi^{\lfloor\varepsilon n\rfloor}\left(T \cdot\left\{\delta_{s}: s \in F\right\}\right)^{n}
$$

for some $\varepsilon>0, T \in \mathcal{B}_{A}$. Using the definition of the convolution, we may rewrite the latter set as

$$
\bigcup_{n=1}^{\infty} \bigcup_{s_{1}, \ldots, s_{n} \in F} \pi^{\lfloor\varepsilon n\rfloor} \cdot T \cdot \alpha_{s_{1}}(T) \cdot \alpha_{s_{1} s_{2}}(T) \cdots \alpha_{s_{1} \cdots s_{n-1}}(T) \delta_{s_{1} \cdots s_{n}} .
$$

The resulting $V$-module is the sum $\sum_{s \in S} U_{s} \delta_{s}$, where $U_{s}$ is the $V$-submodule of $A$ generated by finite products

$$
\left\{\pi^{\lfloor\varepsilon n\rfloor} \cdot T \cdot \alpha_{s_{1}}(T) \cdots \alpha_{s_{1} \cdots s_{n-1}}(T): n \in \mathbb{N}_{\geq 1}, s_{1}, \ldots, s_{n} \in F, s_{1} \cdots s_{n}=s\right\} .
$$

Here taking a factor $1 \in T$ is allowed. Thus we may leave out a factor $\alpha_{s_{1} \cdots s_{i}}(T)$. This has the same effect as increasing $n$ by 1 and putting $s_{i}=s_{i}^{1} \cdot s_{i}^{2}$ with $s_{i}^{1}, s_{i}^{2} \in F$. Since $F$ generates $S$ as a monoid, we may allow arbitrary $s_{i} \in S$ when we change the exponent of $\pi$ appropriately. Namely, we must then replace $n$ in the exponent of $\pi$ by the number of factors in $F$ that are needed to produce the desired elements $s_{i}$, which is $\ell_{\geq 1}\left(s_{1}\right)+\cdots+\ell_{\geq 1}\left(s_{n}\right)$, where $\ell_{\geq 1}(1)=1$ and $\ell_{\geq 1}(s)=\ell(s)$ for $s \in S \backslash\{1\}$. As a result, $U_{s}$ is the $V$-submodule of $A$ generated by

$$
\begin{aligned}
& \pi^{\left\lfloor\varepsilon\left(\ell_{\geq 1}\left(s_{1}\right)+\cdots+\ell_{\geq 1}\left(s_{n}\right)\right)\right\rfloor} \cdot x_{0} \cdot \alpha_{s_{1}}\left(x_{1}\right) \cdots \alpha_{s_{1} \cdots s_{n-1}}\left(x_{n-1}\right), \\
& \quad n \in \mathbb{N}_{\geq 1}, x_{0}, \ldots, x_{n-1} \in T, s_{1}, \ldots, s_{n} \in S, s_{1} \cdots s_{n}=s .
\end{aligned}
$$

Now assume that $S$ is a group, not just a monoid. Then any sequence of elements $g_{1}, \ldots, g_{n} \in S$ may be written as $g_{i}=s_{1} \cdots s_{i}$ by putting $s_{i}:=g_{i-1}^{-1} g_{i}$ with $g_{0}:=1$. So $U_{g}$ is the $V$-submodule of $A$ generated by

$$
\begin{aligned}
\pi^{\left\lfloor\left(\ell_{\geq 1}\left(g_{0}^{-1} g_{1}\right)+\ell_{\geq 1}\left(g_{1}^{-1} g_{2}\right)+\cdots+\ell_{\geq 1}\left(g_{n-1}^{-1} g_{n}\right)\right)\right\rfloor} \cdot \alpha_{g_{0}}\left(x_{0}\right) \cdot \alpha_{g_{1}}\left(x_{1}\right) \cdots \alpha_{g_{n-1}}\left(x_{n-1}\right), \\
n \in \mathbb{N}_{\geq 1}, x_{0}, \ldots, x_{n-1} \in T, g_{0}, \ldots, g_{n} \in S, g_{0}=1, g_{n}=g .
\end{aligned}
$$


These subsets $U_{g}$ for fixed $T$ and $\varepsilon$ depend on $g$ in a complicated way. The bornology on $A \rtimes G$ generated by these subsets is, however, also generated by the sets of the form $\sum_{g \in G} \pi^{\varepsilon \ell(g)} \cdot U \delta_{g}$, where $U$ is the $V$-submodule of $A$ generated by

$$
\begin{aligned}
& \pi^{\left\lfloor\varepsilon\left(\ell_{\geq 1}\left(g_{0}^{-1} g_{1}\right)+\ell_{\geq 1}\left(g_{1}^{-1} g_{2}\right)+\cdots+\ell_{\geq 1}\left(g_{n-2}^{-1} g_{n-1}\right)\right\rfloor\right.} \cdot \alpha_{g_{0}}\left(x_{0}\right) \cdot \alpha_{g_{1}}\left(x_{1}\right) \cdots \alpha_{g_{n-1}}\left(x_{n-1}\right), \\
& n \in \mathbb{N}_{\geq 1}, x_{0}, \ldots, x_{n-1} \in T, g_{0}, \ldots, g_{n-1} \in S, g_{0}=1,
\end{aligned}
$$

for some $T \in \mathcal{B}_{A}, \varepsilon>0$. The reason is that

$$
\ell\left(g_{n}\right)-\ell\left(g_{n-1}\right) \leq \ell\left(g_{n-1}^{-1} g_{n}\right) \leq \ell\left(g_{n-1}\right)+\ell\left(g_{n}\right)
$$

and $\ell\left(g_{n-1}\right) \leq \sum_{j=1}^{n-1} \ell\left(g_{j-1}^{-1} g_{j}\right)$. Therefore, replacing the exponents of $\pi$ as above does not change the bornology on $A \rtimes G$ that is generated by the sets above when $\varepsilon>0$ varies. 



\title{
CHAPTER 2
}

\section{Non-archimedean analytic cyclic homology}

\begin{abstract}
Let $V$ be a complete discrete valuation ring with fraction field $F$ of characteristic zero and with residue field $\mathbb{F}$. We introduce analytic cyclic homology of complete torsion-free bornological algebras over $V$. We prove that it is homotopy invariant, stable, invariant under certain nilpotent extensions, and satisfies excision. We use these properties to compute it for tensor products with dagger completions of Leavitt path algebras. If $R$ is a smooth commutative $V$-algebra of relative dimension 1 , then we identify the analytic cyclic homology of its dagger completion with Berthelot's rigid cohomology of $R \otimes_{V} \mathbb{F}$.
\end{abstract}

\section{Introduction}

Analytic cyclic homology of complete bornological algebras over $\mathbb{R}$ and $\mathbb{C}$ was introduced in $[\mathbf{2 4}$ as a bivariant generalisation from Banach to bornological algebras of the entire cyclic cohomology defined by Connes $\mathbf{9}$ and further studied by Khalkhali 22. It was shown to be stable under tensoring with algebras of nuclear operators and invariant under differentiable homotopies and under analytically nilpotent extensions and to satisfy excision with respect to semi-split extensions $[\mathbf{2 6}]$.

Let $V$ be a complete discrete valuation ring whose fraction field $F$ has characteristic zero. Let $\pi$ be a uniformiser and let $\mathbb{F}:=V / \pi V$ be the residue field. In this article, we define and study an analytic cyclic homology theory for complete, torsion-free bornological $V$-algebras (see Section 2 for the definitions of these terms). For example, if $R$ is a torsion-free, finitely generated, commutative $V$-algebra, then its Monsky-Washnitzer dagger completion $R^{\dagger}$ introduced in $[\mathbf{2 8}$ is such a complete bornological algebra (see [11,27]).

We prove that analytic cyclic homology is invariant under dagger homotopies and under certain nilpotent extensions, that it is matrix stable, and that it satisfies excision with respect to semi-split extensions. We use these properties to compute the analytic cyclic homology for dagger completed Leavitt and Cohn path algebras of countable graphs. For finite graphs, we also compute the analytic cyclic homology for tensor products with such algebras. In particular, it follows that the analytic cyclic homology of the completed tensor product of $R$ with $V\left[t, t^{-1}\right]^{\dagger}$ is isomorphic to the direct sum $\mathrm{HA}_{*}(R) \oplus \mathrm{HA}_{*}(R)[1]$, where $\mathrm{HA}_{*}$ denotes analytic cyclic homology. This is a variant of the fundamental theorem in algebraic K-theory.

We also compute $\mathrm{HA}_{*}\left(R^{\dagger}\right)$ for a smooth, commutative $V$-algebra $R$ of relative dimension 1. Namely, it is isomorphic to the de Rham cohomology of $R^{\dagger}$. If $\mathbb{F}$ has finite characteristic, then this agrees with Berthelot's rigid cohomology of $R \otimes \mathbb{F}$ (see [11]). Partial results that we have for smooth, commutative $V$-algebras of higher dimension have not been included because we have not been able to prove that analytic and periodic cyclic homology coincide in this generality.

Monsky-Washnitzer cohomology and Berthelot's rigid cohomology are defined for varieties in finite characteristic by lifting them to characteristic zero. In order to 
define analogous theories for noncommutative $\mathbb{F}$-algebras, it is natural to replace de Rham cohomology by cyclic homology. Indeed, in 11], Berthelot's rigid cohomology for commutative $\mathbb{F}$-algebras is linked to the periodic cyclic homology of suitable dagger completed commutative $V$-algebras. When we allow noncommutative algebras, however, then the dagger completion process forces us to replace periodic cyclic homology by the analytic cyclic homology that is studied here.

In work in progress, we are going to use the theory defined in this article in order to define an analytic cyclic homology theory for algebras over the residue field $\mathbb{F}$. We want to prove $\mathrm{HA}_{*}(A) \cong \mathrm{HA}_{*}\left(R^{\dagger}\right)$ whenever $R$ is a torsion-free $V$-algebra and $A \cong R / \pi R$ is its reduction to an $\mathbb{F}$-algebra. The crucial point is that this should not depend on the choice of $R$ - and this is where we need analytic instead of periodic cyclic homology.

All theorems in this paper require the fraction field $F$ to have characteristic 0 . This is needed for the homotopy invariance of analytic cyclic homology because the proof involves integration of polynomials. Our proofs of the excision theorem and of matrix stability use characteristic 0 indirectly because they are based on homotopy invariance. Variants of periodic cyclic homology such as (negative) cyclic homology are not homotopy invariant. This is why our methods do not apply to these theories.

Several groups of authors have recently been studying cohomology theories for varieties in finite characteristic with different approaches. We mention, in particular, the work of Petrov and Vologodsky $[\mathbf{2 9}$ that uses topological cyclic homology.

This paper is organised as follows. Some notational conventions used throughout the article are reviewed at the end of this introduction.

In Section 2, we recall some basic notions from bornological analysis and from the Cuntz-Quillen approach to cyclic homology theories. In particular, we introduce dagger completions relative to an ideal (Section 2.2) and review the appropriate notions of extension of bornological modules, noncommutative differential forms, tensor algebra, and $X$-complex for bornological algebras.

Section 3 introduces the analytic cyclic pro-complex $\mathbb{H} \mathbb{A}(R)$ of a complete, torsion-free bornological algebra $R$. It is defined as the $X$-complex of the scalar extension $\mathcal{T} R \otimes_{V} F$ of a certain projective system $\mathcal{T} R$ of complete bornological $V$-algebras functorially associated to $R$. Hence, by definition, $\mathbb{H} \mathbb{A}(R)$ is a prosupercomplex (that is, a projective system of $\mathbb{Z} / 2$-graded chain complexes) of complete bornological vector spaces over $F$. The analytic cyclic homology of $R$ is defined as the homology of the homotopy limit of $\mathbb{H} \mathbb{A}(R)$,

$$
\mathrm{HA}_{*}(R):=H_{*}(\operatorname{holim} \mathbb{H} \mathbb{A}(R)) .
$$

By definition, this is a $\mathbb{Z} / 2$-graded bornological vector space over $F$.

The results about excision, homotopy invariance and matrix stability in this article are all about $\mathbb{H} \mathbb{A}$ as a functor to the homotopy category of chain complexes of projective systems of complete bornological $F$-vector spaces. Here "homotopy category" means that we take chain homotopy classes of chain maps as arrows. It seems, however, that we must pass to a suitable derived category for results that compare $\mathbb{H} \mathbb{A}$ for two different liftings of an algebra over the residue field $\mathbb{F}$. We do not discuss here which weak equivalences must be inverted in order to get a well defined theory for algebras over $\mathbb{F}$. 
Section 4 is concerned with analytic nilpotence. Analytically nilpotent proalgebras and analytically nilpotent extensions of algebras and pro-algebras are introduced. A pro-algebra $R$ is called analytically quasi-free if every semi-split, analytically nilpotent extension of $R$ splits. In particular, the analytic tensor proalgebra $\mathcal{T} R$ (see Definition 4.4.1) is analytically quasi-free and is part of a semi-split, analytically nilpotent extension

$$
\mathcal{J} R \nrightarrow \mathcal{T} R \rightarrow R .
$$

We define dagger homotopy of (pro-)algebra homomorphisms using the dagger completion $V[t]^{\dagger}$, and we show that any semi-split analytically nilpotent extension $N \nrightarrow E \rightarrow R$ with analytically quasi-free $E$ is dagger homotopy equivalent to the extension above. We use this and the invariance of the $X$-complex under dagger homotopies to show that HA is invariant under dagger homotopies. This implies that HA is invariant under analytically nilpotent extensions and that $\mathbb{H} \mathbb{A}(R)$ is homotopy equivalent to $X(R \otimes F)$ if $R$ is analytically quasi-free.

Section 5 is devoted to the proof of the Excision Theorem, which says that if

$$
K \stackrel{i}{\rightarrow} E \stackrel{p}{\rightarrow} Q
$$

is a semi-split pro-algebra extension, then there is a natural exact triangle

$$
\mathbb{H} \mathbb{A}(K) \stackrel{i}{\rightarrow} \mathbb{H} \mathbb{A}(E) \stackrel{p}{\rightarrow} \mathbb{H} \mathbb{A}(Q) \stackrel{\delta}{\rightarrow} \mathbb{H} \mathbb{A}(K)[-1] .
$$

Applying the homotopy projective limit and taking homology, this implies a natural 6 -term exact sequence

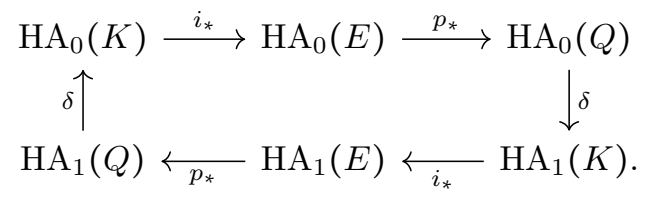

The proof of the excision theorem follows the structure of its archimedean version in $\mathbf{2 5} \mathbf{2 6}$, and adapts it to the present case.

The stability of HA under matricial embeddings is proved in Section 6 Any pair $X, Y$ of torsion-free bornological $V$-modules with a surjective bounded linear map $\langle\cdot, \cdot\rangle: Y \otimes X \rightarrow V$ gives rise to a dagger algebra $\overline{\mathcal{M}}(X, Y)$ with underlying bornological $V$-module $X \otimes Y$. We show in Proposition 6.2 that HA is invariant under tensoring with $\overline{\mathcal{M}}(X, Y)$. For example, the algebra of finite matrices $\mathbb{M}_{n}$ with $n \leq \infty$ and the algebra of matrices with entries going to zero at infinity are of the form $\overline{\mathcal{M}}(X, Y)$ for suitable $X$ and $Y$. Thus HA is invariant under tensoring with such algebras. This implies that HA for unital algebras is functorial for certain bimodules and invariant under Morita equivalence (see Section 7).

Section 8 is concerned with Leavitt path algebras. For a directed graph $E$ with finitely many vertices and a complete bornological algebra $R$, Theorems 8.1 and 8.3 compute $\mathrm{HA}\left(R \otimes L(E)^{\dagger}\right)$ in terms of $\mathrm{HA}(R)$ and a matrix $N_{E}$ related to the incidence matrix of $E$ :

$$
\mathbb{H} \mathbb{A}\left(R \otimes L(E)^{\dagger}\right) \simeq\left(\operatorname{coker}\left(N_{E}\right) \oplus \operatorname{ker}\left(N_{E}\right)[1]\right) \otimes \mathbb{H} \mathbb{A}(R) .
$$

For trivial $R$, the homotopy equivalence $\mathbb{H} \mathbb{A}\left(L(E)^{\dagger}\right) \simeq\left(\operatorname{coker}\left(N_{E}\right) \oplus \operatorname{ker}\left(N_{E}\right)[1]\right)$ is shown also for graphs with countably many vertices. If $E$ is the graph with one 
vertex and one loop, it follows that HA satisfies a version of Bass' fundamental theorem:

$$
\mathbb{H} \mathbb{A}\left(R \otimes V\left[t, t^{-1}\right]^{\dagger}\right) \simeq \mathbb{H} \mathbb{A}(R) \oplus \mathbb{H} \mathbb{A}(R)[-1] .
$$

We also compute $\mathbb{H} \mathbb{A}\left(R \bar{\otimes} C(E)^{\dagger}\right)$ for the Cohn path algebra of $E$ if $E$ has finitely many vertices, and $\mathbb{H} \mathbb{A}\left(C(E)^{\dagger}\right)$ if $E$ has countably many vertices.

In Section 9 we show that if $R$ is smooth commutative of relative dimension one, then the analytic cyclic homology of its dagger completion is the same as the rigid cohomology of its reduction modulo $\pi$ (see Theorem 9.2.9). That is,

$$
\mathrm{HA}_{n}\left(R^{\dagger}\right) \cong H_{\text {rig }}^{n}(R / \pi R)
$$

for $n=0,1$. We outline the idea of the proof. By 11, $H_{\mathrm{rig}}^{n}(R / \pi R)$ is isomorphic to the periodic cyclic homology of $R^{\dagger} \otimes F$. By Corollary 4.7.2. HA and $\operatorname{HP}(\cdot \otimes F)$ agree on analytically quasi-free bornological $V$-algebras. It is well known that a smooth algebra $R$ of relative dimension 1 is quasi-free in the sense that any square-zero extension of $R$ splits or, equivalently, that the bimodule $\Omega^{1}(R)$ of noncommutative differential 1-forms admits a connection. We show in Theorem 9.1.9 that if $R$ is a torsion-free, complete bornological algebra and $\nabla$ is a connection on $\Omega^{1}(R)$ that satisfies an extra condition, then $R^{\dagger}$ is analytically quasi-free. We prove that a smooth commutative algebra of relative dimension 1 with the fine bornology admits such a connection (see Lemma 9.2.3.

Acknowledgement. A first draft of this manuscript was completed during a 6month stay of the first author at the Universität Münster, supported by a Humboldt Research Award. He thanks his host, Joachim Cuntz and all the members of the Operator Algebra group, particularly its head, Wilhelm Winter, for their wonderful hospitality. He also thanks the Humboldt Foundation for its support.

1.1. Some notation. Throughout this article, we shall use the following notation. Let $\mathbb{N}^{*}$ be the set of nonzero natural numbers. Let $V$ be a complete discrete valuation ring, $\pi \in V$ a uniformiser, $\mathbb{F}$ the residue field $V /(\pi)$ of $V$, and $F$ the fraction field of $V$. While our definitions work in complete generality, our homotopy invariance, stability and excision theorems only work if $F$ has characteristic zero. All tensor products $\otimes$ are taken over $V$. By convention, algebras are allowed to be non-unital throughout this article. An ideal in a possibly non-unital $V$-algebra means a two-sided ideal that is also a $V$-submodule.

\section{Preparations}

2.1. Bornologies. As in [11], bornological $V$-algebras play a crucial role. We first recall some basic terminology about bornologies from [11, 27].

Definition 2.1.1. A bornology on a set $S$ is a set $\mathcal{B}$ of subsets, called bounded subsets, such that finite unions and subsets of bounded subsets are bounded and finite subsets are bounded. A bornological set is a set with a bornology.

Definition 2.1.2. A map $f: S_{1} \rightarrow S_{2}$ between bornological sets is bounded if it maps bounded subsets to bounded subsets. It is a bornological embedding if it is injective and $T \subseteq S_{1}$ is bounded if and only if $f(T) \subseteq S_{2}$ is bounded. It is a bornological quotient map if it is bounded and any bounded subset $T \subseteq S_{2}$ is the image of a bounded subset of $S_{1}$. 
Definition 2.1.3. A bornological $V$-module is a $V$-module $R$ with a bornology such that any bounded subset is contained in a bounded $V$-submodule or, equivalently, the $V$-submodule generated by a bounded subset is again bounded. A bornological $V$-algebra is a bornological $V$-module $R$ with a multiplication $R \times R \rightarrow R$ that is bounded in the sense that $S \cdot T$ is bounded if $S, T \subseteq R$ are bounded.

Definition 2.1.4. A bornological $V$-module is complete if any bounded subset is contained in a bounded $V$-submodule that is $\pi$-adically complete. The completion $\vec{M}$ of a bornological $V$-module $M$ is a complete bornological $V$-module with a bounded map $M \rightarrow T$ that is universal in the sense that any bounded map from $M$ to a complete bornological $V$-module factors uniquely through it (see 11. Definition 2.14]).

ExAmple 2.1.5. Let $M$ be a $V$-module. The fine bornology on $M$ consists of those subsets of $M$ that are contained in a finitely generated $V$-submodule. It is the smallest $V$-module bornology on $M$. It is the only bornology on $M$ if $M$ itself is finitely generated. If $R$ is a $V$-algebra, then the fine bornology makes it a bornological $V$-algebra. The fine bornology is automatically complete.

We always equip the fraction field $F$ with the fine bornology.

Definition 2.1.6. Let $M_{1}$ and $M_{2}$ be bornological $V$-modules. The tensor product bornology on the $V$-module $M_{1} \otimes M_{2}$ consists of all subsets that are contained in $S_{1} \otimes S_{2}$ for bounded $V$-submodules $S_{j} \subseteq M_{j}$ for $j=1,2$. The complete bornological tensor product $M_{1} \otimes M_{2}$ is defined as the bornological completion of $M_{1} \otimes M_{2}$ with the tensor product bornology.

The universal property of tensor products easily implies the following:

Proposition 2.1.7. Let $M_{1}, M_{2}$ and $N$ be bornological $V$-modules. Bounded $V$-linear maps $M_{1} \otimes M_{2} \rightarrow N$ are in natural bijection with bounded $V$-bilinear maps $M_{1} \times M_{2} \rightarrow N$.

Corollary 2.1.8. Let $M_{1}, M_{2}$ and $N$ be complete bornological $V$-modules. Bounded $V$-linear maps $M_{1} \otimes M_{2} \rightarrow N$ are in natural bijection with bounded $V$-bilinear maps $M_{1} \times M_{2} \rightarrow N$.

Example 2.1.9. Continuing Example 2.1.5, let $M_{1}$ be a $V$-module with the fine bornology and let $M_{2}$ be a complete bornological $V$-module. Then the tensor product bornology on $M_{1} \otimes M_{2}$ is already complete because the tensor product of a $\pi$-adically complete $V$-module with a finitely generated $V$-module is complete. Thus $M_{1} \otimes M_{2}=M_{1} \otimes M_{2}$ in this case. This applies, in particular, if $M_{1}=F$. If both $M_{1}$ and $M_{2}$ carry the fine bornology, then the tensor product bornology on $M_{1} \otimes M_{2}=M_{1} \otimes M_{2}$ is the fine bornology as well.

Definition 2.1.10 ([27, Definition 4.1]). A bornological $V$-module is (bornologically) torsion-free if multiplication by $\pi$ is a bornological embedding.

Remark 2.1.11. Let $M$ be a bornological $V$-module. If $S \subseteq M$, then define

$$
\pi^{-1} S:=\{x \in M: \pi \cdot x \in S\} .
$$

This depends on $M$ and not just on $S$. By definition, $M$ is torsion-free if and only if multiplication by $\pi$ is injective and $\pi^{-1} S$ is bounded for all bounded subsets $S \subseteq M$.

Proposition 2.1.12 ([27, Proposition 4.3]). A bornological $V$-module $M$ is torsion-free if and only if the canonical map $M \rightarrow M \otimes F$ is a bornological embedding. 
ExAmPLE 2.1.13. A $V$-module $M$ with the fine bornology is torsion-free if and only if $M$ is torsion-free in the usual sense.

Definition 2.1.14. Let $M$ be any bornological $V$-module and define $M_{\mathrm{tf}} \subseteq$ $M \otimes F$ as the image of the canonical map $M \rightarrow M \otimes F$, equipped with the restriction of the bornology of $M \otimes F$.

Proposition 2.1.15 ( $\left[\mathbf{2 7}\right.$, Proposition 4.4]). The canonical map $M \rightarrow M_{\mathrm{tf}}$ is the universal map from $M$ to a torsion-free bornological $V$-module.

Definition 2.1.16. A bornological $V$-algebra $R$ is semi-dagger if any bounded subset $S \subseteq R$ is contained in a bounded $V$-submodule $T \subseteq R$ with $\pi \cdot T \cdot T \subseteq T$ (see 27. Proposition 3.4]). Let $R$ with the bornology $\mathcal{B}$ be a bornological $V$-algebra. There is a smallest semi-dagger bornology on $R$ that contains $\mathcal{B}$. It is denoted $\mathcal{B}_{\lg }$ and called the linear growth bornology on $R$; we write $R_{\lg }$ for $R$ with the linear growth bornology (see [27, Definition 3.5 and Lemma 3.6]).

Definition 2.1.17. A dagger algebra is a bornological $V$-algebra that is complete, (bornologically) torsion-free, and semi-dagger. The dagger completion of a bornological $V$-algebra $R$ is a dagger algebra $R^{\dagger}$ with a bounded $V$-algebra homomorphism $R \rightarrow R^{\dagger}$ that is universal in the sense that any bounded homomorphism from $R$ to a dagger algebra factors uniquely through it.

TheOREM 2.1.18 ( $\mathbf{2 7}$, Theorem 5.3]). If $R$ is already torsion-free, then $R^{\dagger}$ is the completion of $R_{\mathrm{lg}}$. In general, it is the completion of $\left(R_{\mathrm{tf}}\right)_{\mathrm{lg}}$.

ExAmPLE 2.1.19. The dagger completion $R^{\dagger}$ of a torsion-free, finitely generated, commutative $V$-algebra is usually defined as the weak completion of $R$ by Monsky and Washnitzer $2 \mathbf{2 8}$. This agrees with our definition of $R^{\dagger}$ by $[\mathbf{1 1}$, Theorem 3.2.1]: the dagger completion of the fine bornology on $R$ is naturally isomorphic to the weak completion of $R$, equipped with a canonical bornology.

Proposition 2.1.20 ( $[\mathbf{1 1}$, Proposition 3.1.25]). Let $A$ and $B$ be torsion-free, complete bornological algebras. Then $(A \otimes B)_{\lg } \cong A_{\lg } \otimes B_{\lg }$ and $(A \otimes B)^{\dagger} \cong A^{\dagger} \otimes B^{\dagger}$.

COROLlaRY 2.1.21. A completed tensor product of two dagger algebras is again a dagger algebra.

ProOF. A completed tensor product is complete by definition. It remains semi-dagger by Proposition 2.1.20, and torsion-free by [27, Proposition 4.12].

2.2. Relative dagger completions. We shall define analytic cyclic homology for torsion-free, complete bornological $V$-algebras $R$ that need not be dagger algebras. This uses a variant of the linear growth bornology relative to an ideal.

Let $R$ be a $V$-algebra and let $M$ and $N$ be $V$-submodules of $R$. Let $M N \subseteq R$ be the $V$-submodule generated by all products $x y$ with $x \in M$ and $y \in N$. Let

$$
M^{\diamond}:=\sum_{i=0}^{\infty} \pi^{i} M^{i+1}, \quad M^{(n)}:=\sum_{i=1}^{n} M^{i} .
$$

A subset of $R$ has linear growth if and only if it is contained in $M^{\diamond}$ for some bounded $V$-submodule $M$ of $R$ (with the present definitions, this is [27, Lemma 3.6]).

Lemma 2.2.2. Let $R$ be a $V$-algebra and let $M, N \subseteq R$ be $V$-submodules. Then (1) $M^{\diamond}+N^{\diamond} \subseteq(M+N)^{\diamond}$; 
(2) $M \cdot N^{\diamond} \subseteq\left((M \cdot N+N)^{(2)}\right)^{\diamond}$ and $N^{\diamond} \cdot M \subseteq\left((N \cdot M+N)^{(2)}\right)^{\diamond}$;

(3) $\pi \cdot M^{\diamond} \cdot M^{\diamond} \subseteq M^{\diamond}$;

(4) $M^{\diamond} \cdot N^{\diamond} \subseteq\left((M+N)^{(2)}\right)^{\diamond}$;

(5) $\left(M^{\diamond}\right)^{\diamond}=M^{\diamond}$.

Proof. The definition of $M^{\diamond}$ immediately implies (1). The following computation shows the first assertion of (2)

$$
\begin{aligned}
M \cdot N^{\diamond} & =\sum_{i \geq 1} \pi^{2 i-1} M N^{2 i}+\sum_{i \geq 0} \pi^{2 i} M N^{2 i+1} \\
& =\sum_{i \geq 1} \pi^{2 i-1}(M N) N^{2 i-1}+\sum_{i \geq 0} \pi^{2 i}(M N)\left(N^{2}\right)^{i} \\
& \subseteq(M N+N)^{\diamond}+\left(M N+N^{2}\right)^{\diamond} \subseteq\left((M N+N)^{(2)}\right)^{\diamond} .
\end{aligned}
$$

Similar calculations give the second assertion of (2) and (4) Statement (3) follows because $\pi \cdot \pi^{i} M^{i+1} \cdot \pi^{j} M^{j+1}=\pi^{i+j+1} M^{i+j+1+1}$ for all $i, j \in \mathbb{N}$. Then $\pi^{i} \cdot\left(M^{\diamond}\right)^{\imath+1} \subseteq M^{\diamond}$ follows by induction on $i$. This implies (5)

Definition 2.2.3. Let $R$ be a bornological $V$-algebra and $I \triangleleft R$ an ideal. Let $\mathcal{B}_{\lg (I)}$ be the set of all subsets of $R$ that are contained in $M+N^{\diamond}$ for bounded $V$-submodules $M \subseteq R$ and $N \subseteq I$. This is a bornology on $R$, called the linear growth bornology relative to $I$. Let $R_{\lg (I)}$ be $R$ with this bornology.

EXAmple 2.2.4. By definition, $\mathcal{B}_{\lg (0)}=\mathcal{B}$ and $\mathcal{B}_{\lg (R)}$ is the usual linear growth bornology on $R$. So $R_{\lg (0)}=R$ and $R_{\lg (R)}=R_{\lg }$.

LEMMA 2.2.5. The bornology $\mathcal{B}_{\lg (I)}$ is an algebra bornology, and its restriction to $I$ is semi-dagger. Let $S$ be a bornological $V$-algebra. A homomorphism $f: R \rightarrow S$ is bounded for the bornology $\mathcal{B}_{\lg (I)}$ if and only if $f(N)$ has linear growth in $S$ for all bounded subsets $N \subseteq I$ and $f(M)$ is bounded in $S$ for all bounded subsets $M \subseteq R$.

Proof. Since $I$ is an ideal, Lemma 2.2 .2 implies that $\mathcal{B}_{\lg (I)}$ makes $R$ a bornological $V$-algebra. And a subset of $I$ belongs to $\mathcal{B}_{\lg (I)}$ if and only if it is contained in $N^{\diamond}$ for some bounded $V$-submodule $N \subseteq I$. The restriction of $\mathcal{B}_{\lg (I)}$ to $I$ is semi-dagger by Lemma 2.2.2 If $M$ and $N$ are as in Definition 2.2.3, then $f\left(M+N^{\diamond}\right)=f(M)+f(N)^{\diamond}$. This is bounded in $S$ if and only if $f(M)$ is bounded and $f(N)$ has linear growth.

Lemma 2.2.6. Let $R$ be a bornological algebra and let $I$ and $J$ be ideals in $R$ with $I \subseteq J$ and $R / I=(R / I)_{\lg (J / I)}$. Then $R_{\lg (J)}=R_{\lg (I)}$. In particular, if $R / I$ is semi-dagger, then $R_{\lg (I)}=R_{\lg }$.

Proof. By Lemma 2.2.5, the bornology $\mathcal{B}_{\lg (J)}$ on $R$ is the smallest one that contains the given bornology and makes $J$ semi-dagger, and similarly for $I$. And the assumption $R / I=(R / I)_{\lg (J / I)}$ says that $J / I \subseteq R / I$ is semi-dagger in the quotient bornology on $R / I$. This is the same as the quotient bornology induced by $\mathcal{B}_{\lg (I)}$. [27. Theorem 3.7] says that an extension of semi-dagger algebras remains semidagger. This theorem applied to the extension $I \rightarrow J \rightarrow J / I$, equipped with the restrictions of the bornology $\mathcal{B}_{\lg (I)}$ on $I$ and $J$ and the resulting quotient bornology on $J / I$ shows that $J$ is semi-dagger also in the bornology $\mathcal{B}_{\lg (I)}$. Then $\mathcal{B}_{\lg (J)} \subseteq \mathcal{B}_{\lg (I)}$. And $\mathcal{B}_{\lg (I)} \subseteq \mathcal{B}_{\lg (J)}$ is trivial.

Lemma 2.2.7. Let $R$ be a bornological algebra and $I \triangleleft R$ an ideal. If $R$ is torsion-free, then so is $R_{\lg (I)}$. 
Proof. Let $S \subseteq \pi R$ be a bounded subset in $R_{\lg (I)}$. By definition, there are bounded submodules $M \subseteq R$ and $N \subseteq I$ with $S \subseteq M+N^{\diamond}$. And

$$
M+N^{\diamond}=M+N+\sum_{i=1}^{\infty} \pi^{i} N^{i+1}=M+N+\pi \cdot\left(\sum_{i=0}^{\infty} \pi^{i} N^{i+2}\right) .
$$

Since $\pi^{i} N^{i+2} \subseteq \pi^{i}\left(N^{(2)}\right)^{i+1}$ for all $i \geq 0$, the subset $\sum_{i=0}^{\infty} \pi^{i} N^{i+2}$ belongs to $\mathcal{B}_{\lg (I)}$. Since $M+N$ is bounded in $R$ and $R$ is torsion-free, $\pi^{-1} \cdot(M+N)$ is bounded. Then

$$
\pi^{-1} S \subseteq \pi^{-1}\left(M+N^{\diamond}\right) \subseteq \pi^{-1}(M+N)+\sum_{i=0}^{\infty} \pi^{i} N^{i+2} \in \mathcal{B}_{\lg (I)}
$$

DeFinition 2.2.8. Let $R$ be a torsion-free bornological algebra and $I \triangleleft R$ an ideal. The dagger completion of $R$ relative to $I$ is the completion $(R, I)^{\dagger}:=\overline{R_{\lg (I)}}$.

We shall never apply (relative) dagger completions when $R$ is not already bornologically torsion-free. In general, the correct definition of the relative dagger completion of $(R, I)$ would be $\left(R_{\mathrm{tf}}, I_{\mathrm{tf}}\right)^{\dagger}$, where $I_{\mathrm{tf}}$ is identified with its image in $R_{\mathrm{tf}}$ (compare Theorem 2.1.18).

Proposition 2.2.9. Let $R$ and $S$ be torsion-free bornological $V$-algebras, $I \subseteq R$ an ideal, and $f: R \rightarrow S$ a bounded algebra homomorphism. Assume $S$ to be complete. There is a bounded algebra homomorphism $(R, I)^{\dagger} \rightarrow S$ extending $f$-necessarily unique - if and only if $f(M)$ has linear growth for each bounded $V$-submodule $M$ of $I$.

Proof. Use Lemma 2.2.5 and the universal property of the completion.

We know no analogue of Proposition 2.1.20 for relative dagger completions.

2.3. Extensions of bornological modules. An extension of $V$-modules is a diagram of $V$-modules

$$
K \stackrel{i}{\rightarrow} E \stackrel{p}{\rightarrow} Q
$$

that is algebraically exact and such that $i$ is a bornological embedding and $p$ is a bornological quotient map. Equivalently, $i$ is a kernel of $p$ and $p$ is a cokernel of $i$ in the additive category of bornological $V$-modules. The following elementary lemma says that this category is quasi-abelian (see $\mathbf{3 5}$ ):

Lemma 2.3.2. Let 2.3.1) be an extension of bornological $V$-modules and let $f: K \rightarrow K^{\prime}$ and $g: Q^{\prime \prime} \rightarrow Q$ be bounded $V$-module maps. The pushout of $i, f$ and the pullback of $p, g$ exist and are part of morphisms of extensions
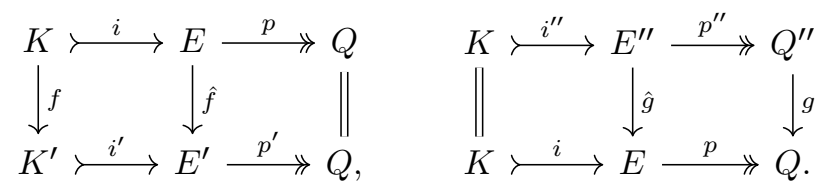

Here

$$
E^{\prime}:=\frac{K^{\prime} \oplus E}{\{(f(k),-i(k)): k \in K\}}, \quad E^{\prime \prime}:=\left\{\left(e, q^{\prime \prime}\right) \in E \times Q^{\prime \prime}: p(e)=g\left(q^{\prime \prime}\right)\right\},
$$

equipped with the quotient and the subspace bornology, respectively, and $\hat{f}(e)=$ $[(0, e)], i^{\prime}\left(k^{\prime}\right)=\left[\left(k^{\prime}, 0\right)\right], p^{\prime}\left[\left(k^{\prime}, e\right)\right]=p(e), \hat{g}\left(e, q^{\prime \prime}\right)=e, p^{\prime \prime}\left(e, q^{\prime \prime}\right)=q^{\prime \prime}$, and $i^{\prime \prime}(k)=$ $(i(k), 0)$ for $e \in E, k^{\prime} \in K^{\prime}, q^{\prime \prime} \in Q^{\prime \prime}, k \in K$. 
The following proposition is an analogue of Lemma 2.2.6 for completions, describing a situation when a partial completion relative to a submodule is equal to the completion.

Proposition 2.3.3. Assume $Q$ in an extension 2.3.1) of bornological $V$-modules to be complete and bornologically torsion-free. Form the pushout diagram

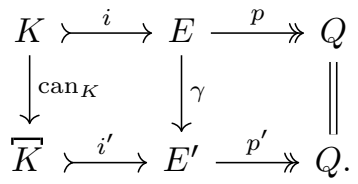

Then there is a unique isomorphism $\varphi: E^{\prime} \stackrel{\sim}{\rightarrow} E$ such that $\varphi \circ \gamma$ is the canonical map $E \rightarrow$ E.

Proof. The bottom row is an extension by Lemma 2.3.2 Then $E^{\prime}$ is complete by 27. Theorem 2.3]. The maps $\operatorname{can}_{E}: E \rightarrow E$ and $i: T \rightarrow E$ induce a bounded $V$-module map $\varphi: E^{\prime} \rightarrow Z$ by the universal property of pushouts. Since $E^{\prime}$ is

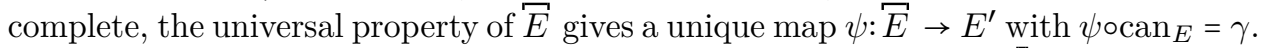
Then $\varphi \circ \psi \circ \operatorname{can}_{E}=\varphi \circ \gamma=\operatorname{can}_{E}$. This implies $\varphi \circ \psi=\mathrm{id}_{E}$. Next, $\psi \circ \bar{i} \circ \operatorname{can}_{K}=\gamma \circ i=$ $i^{\prime} \circ \operatorname{can}_{K}$ implies $\psi \circ \bar{i}=i^{\prime}$, and then $\psi \circ \varphi \circ i^{\prime}=\psi \circ \vec{i}=i^{\prime}$ and $\psi \circ \varphi \circ \gamma=\psi \circ \operatorname{can}_{E}=\gamma$ imply $\psi \circ \varphi=\operatorname{id}_{E^{\prime}}$. So $\varphi$ is an isomorphism.

2.4. Injective maps between completions. Unlike in the Archimedean case, all Banach spaces over $F$ have a simple structure. This implies that they all satisfy a variant of Grothendieck's Approximation Property. This is Proposition 2.4.5. and it will be useful to describe completions of tensor products.

Definition 2.4.1. Let $D$ be a set. Let $\mathrm{C}_{0}(D, V)$ be the $V$-module of all functions $f: D \rightarrow V$ such that for each $\delta>0$ there is a finite subset $S \subseteq D$ with $|f(x)|<\delta$ for all $x \in D \backslash S$. Define $\mathrm{C}_{0}(D, F)$ similarly. Equip $\mathrm{C}_{0}(D, V)$ and $\mathrm{C}_{0}(D, F)$ with the supremum norm.

TheOREM 2.4.2. Any $\pi$-adically complete, torsion-free $V$-module $M$ is isomorphic to $\mathrm{C}_{0}(D, V)$ for some set $D$.

Proof. The map $M \rightarrow M \otimes F$ is an embedding because $M$ is torsion-free. Define the gauge norm on $F \cdot M$ by

$$
\|x\|:=\inf \left\{|\pi|^{j}: \pi^{-j} \cdot x \in M\right\} .
$$

It is a non-Archimedean norm and makes $F \cdot M$ a Banach $F$-vector space with unit ball $M$. It takes values in $\left\{|\pi|^{n}: n \in \mathbb{Z}\right\} \cup\{0\}$ by construction. Hence there is a set $D$ and an isometric isomorphism $F M \cong \mathrm{C}_{0}(D, F)$ (see 34. Remark 10.2]). It maps $M$ isomorphically onto the the unit ball of $\mathrm{C}_{0}(D, F)$, which is $\mathrm{C}_{0}(D, V)$.

Corollary 2.4.3. Let $W$ be a complete, torsion-free bornological $V$-module. Then $W$ is isomorphic to the colimit of an inductive system of complete $V$-modules of the form $\left(\mathrm{C}_{0}\left(D_{n}, V\right), f_{n, m}\right)_{n, m \in S}$ with a directed set $(S, \leq)$, sets $D_{n}$ for $n \in S$, and injective, bounded $V$-linear maps $f_{n, m}: \mathrm{C}_{0}\left(D_{m}, V\right) \rightarrow \mathrm{C}_{0}\left(D_{n}, V\right)$ for $n, m \in S$, $n \geq m$. 
Proof. The complete $V$-submodules of $W$ form a directed set under inclusion. This defines an inductive system with injective structure maps and with colimit $W$ by 11. Proposition 2.10]. Each complete $V$-submodule of $W$ is $\pi$-adically complete and torsion-free. Then it is isomorphic to $\mathrm{C}_{0}(D, V)$ for some set $D$ by Theorem 4.1.2.

Lemma 2.4.4. Let $f: \mathrm{C}_{0}\left(D_{1}, V\right) \rightarrow \mathrm{C}_{0}\left(D_{2}, V\right)$ and $g: \mathrm{C}_{0}\left(D_{3}, V\right) \hookrightarrow \mathrm{C}_{0}\left(D_{4}, V\right)$ be injective, bounded $V$-linear maps. Then the induced bounded map

$$
f \widehat{\otimes} g: \mathrm{C}_{0}\left(D_{1}, V\right) \widehat{\otimes} \mathrm{C}_{0}\left(D_{3}, V\right) \rightarrow \mathrm{C}_{0}\left(D_{2}, V\right) \widehat{\otimes} \mathrm{C}_{0}\left(D_{4}, V\right)
$$

is injective as well. And here $\mathrm{C}_{0}\left(D_{m}, V\right) \widehat{\otimes} \mathrm{C}_{0}\left(D_{n}, V\right) \cong \mathrm{C}_{0}\left(D_{m} \times D_{n}, V\right)$.

Proof. The universal property of the complete bornological tensor product implies that $\mathrm{C}_{0}\left(D_{1}, V\right) \widehat{\otimes} \mathrm{C}_{0}\left(D_{3}, V\right) \cong \mathrm{C}_{0}\left(D_{1} \times D_{3}, V\right)$ for all sets $D_{1}$ and $D_{2}$. Define $\mathrm{C}_{0}\left(D_{1}, \mathrm{C}_{0}\left(D_{3}, V\right)\right)$ to be the space of all functions $f: D_{1} \rightarrow \mathrm{C}_{0}\left(D_{3}, V\right)$ for which the gauge norm $\|f\|$ vanishes at $\infty$. There is a canonical isomorphism

$$
\mathrm{C}_{0}\left(D_{1} \times D_{3}, V\right) \stackrel{\cong}{\rightrightarrows} \mathrm{C}_{0}\left(D_{1}, \mathrm{C}_{0}\left(D_{3}, V\right)\right), \quad f \mapsto(s \mapsto f(s, \cdot)) .
$$

Similarly, $\mathrm{C}_{0}\left(D_{1} \times D_{3}, V\right) \cong \mathrm{C}_{0}\left(D_{3}, \mathrm{C}_{0}\left(D_{1}, V\right)\right)$. Now we factorise the map $f \nexists g$ as

$$
\begin{aligned}
& \mathrm{C}_{0}\left(D_{1}, V\right) \widehat{\otimes} \mathrm{C}_{0}\left(D_{3}, V\right) \cong \mathrm{C}_{0}\left(D_{1} \times D_{3}, V\right) \cong \mathrm{C}_{0}\left(D_{1}, \mathrm{C}_{0}\left(D_{3}, V\right)\right) \\
& \stackrel{g_{*}}{\rightarrow} \mathrm{C}_{0}\left(D_{1}, \mathrm{C}_{0}\left(D_{4}, V\right)\right) \cong \mathrm{C}_{0}\left(D_{4}, \mathrm{C}_{0}\left(D_{1}, V\right)\right) \\
& \stackrel{f_{*}}{\rightarrow} \mathrm{C}_{0}\left(D_{4}, \mathrm{C}_{0}\left(D_{2}, V\right)\right) \cong \mathrm{C}_{0}\left(D_{2} \times D_{4}, V\right) \cong \mathrm{C}_{0}\left(D_{2}, V\right) \widehat{\otimes} \mathrm{C}_{0}\left(D_{4}, V\right) ;
\end{aligned}
$$

here the maps $f_{*}$ and $g_{*}$ are injective because $f$ and $g$ are injective.

Proposition 2.4.5. Let $M_{1}, W_{1}, M_{2}$ and $W_{2}$ be complete, torsion-free bornological $V$-modules and let $\varphi_{j}: M_{j} \hookrightarrow W_{j}$ for $j=1,2$ be injective bounded $V$-module maps. Then $\varphi_{1} \otimes \varphi_{2}: M_{1} \otimes M_{2} \rightarrow W_{1} \otimes W_{2}$ is injective.

Proof. Write $W_{1}$ and $W_{2}$ as inductive limits as in Corollary 2.4.3. Then $W_{1} \otimes W_{2}$ is naturally isomorphic to the inductive limit of the inductive system defined by the maps $f_{1, n_{1}, m_{1}} \otimes f_{2, n_{2}, m_{2}}: \mathrm{C}_{0}\left(D_{n_{1}}, V\right) \otimes \mathrm{C}_{0}\left(J_{n_{2}}, V\right) \rightarrow \mathrm{C}_{0}\left(D_{m_{1}}, V\right) \otimes$ $\mathrm{C}_{0}\left(J_{m_{2}}, V\right)$, and $W_{1} \otimes W_{2}$ is naturally isomorphic to the inductive limit of the inductive system defined by the maps $f_{1, n_{1}, m_{1}} \widehat{\otimes} f_{2, n_{2}, m_{2}}: \mathrm{C}_{0}\left(D_{n_{1}}, V\right) \widehat{\otimes} \mathrm{C}_{0}\left(J_{n_{2}}, V\right) \rightarrow$ $\mathrm{C}_{0}\left(D_{m_{1}}, V\right) \widehat{\otimes} \mathrm{C}_{0}\left(J_{m_{2}}, V\right)$. All these bounded maps are injective by Lemma 2.4.4. Therefore, the tensor product is isomorphic to an ordinary union of these $V$-modules, equipped with the bornology cofinally generated by these $V$-submodules. The tensor products $M_{1} \otimes M_{2}$ and $M_{1} \otimes M_{2}$ are described similarly, and the maps $\varphi_{1}$ and $\varphi_{2}$ are described by injective maps between the entries of the appropriate inductive systems. Then Lemma 2.4.4 shows that $\varphi_{1} \mathbb{\otimes} \varphi_{2}$ is injective.

2.5. The bimodule of differential 1-forms. We are going to define the (complete) bimodule $\widetilde{\Omega}^{1}(A)$ of noncommutative differential 1-forms over a complete bornological $V$-algebra $A$.

For a unital algebra in the usual sense, $\Omega^{1}(A)$ is defined in [16. Section 1] as $A \otimes(A / \mathbb{C} \cdot 1)$ with a certain bimodule structure. Its elements are denoted by $a \mathrm{~d} b$ with $a \in A, b \in A / \mathbb{C} \cdot 1$. We shall use the version for non-unital algebras, which uses $A^{+}$instead of $A$. It is clear from the definition that the map $\Omega^{1}(A) \rightarrow A^{+} \otimes A^{+}$, $a \mathrm{~d} b \mapsto a \otimes b-a b \otimes 1$, is an isomorphism onto the kernel of the multiplication map $A^{+} \otimes A^{+} \rightarrow A^{+}$. In $\left[26\right.$, Appendix A.3], $\Omega^{1}(A)$ is defined as this kernel when $A$ is an algebra in a symmetric monoidal category. The kernel exists and is a direct 
summand as a left or right module because the multiplication map $A^{+} \otimes A^{+} \rightarrow A^{+}$ splits as a left or right $A$-module map. This definition applies in our setting, using the tensor product $\bar{\otimes}$.

By definition, $\Omega^{1}(A) \subseteq A^{+} \Phi A^{+}$is a complete bornological $A$-bimodule. The map

$$
\mathrm{d}: A \rightarrow \bar{\Omega}^{1}(A), \quad \mathrm{d}(x):=1 \otimes x-x \otimes 1,
$$

is the universal bounded derivation into a complete $A$-bimodule, that is, any bounded derivation $\partial: A \rightarrow M$ into a complete $A$-bimodule factors uniquely through d. Namely, there is a unique bounded bimodule homomorphism $\widetilde{\Omega}^{1}(A) \rightarrow M, a_{0} \mathrm{~d} a_{1} \mapsto a_{0} \cdot \partial\left(a_{1}\right)$. This factorisation exists because there are bornological isomorphisms

$$
\begin{array}{ll}
A^{+} \otimes A \rightarrow \widetilde{\Omega}^{1}(A), & x \otimes y \mapsto x \mathrm{~d} y, \\
A \Phi A^{+} \rightarrow \widetilde{\Omega}^{1}(A), & x \otimes y \mapsto(\mathrm{d} x) \cdot y=\mathrm{d}(x \cdot y)-x \mathrm{~d} y .
\end{array}
$$

The first one is left and the second one right $A$-linear.

We now relate $\bar{\Omega}^{1}(A)$ to sections of semi-split, square-zero extensions of $A$ (see [26. Theorem A.53] or [16, Proposition 3.3]). Let $M$ be a complete bornological $A$-bimodule. Give $A \oplus M$ the multiplication

$$
\left(a_{1}, m_{1}\right) \cdot\left(a_{2}, m_{2}\right):=\left(a_{1} \cdot a_{2}, a_{1} \cdot m_{2}+m_{1} \cdot a_{2}\right) .
$$

The inclusion $M \nrightarrow A \oplus M$ and the projection $A \oplus M \rightarrow A$ form a square-zero extension that splits by the inclusion homomorphism $A \leftrightarrow A \oplus M$.

LemMa 2.5.1. Let $A$ be a complete bornological algebra and let $M$ be a complete bornological A-bimodule. There is a natural bijection between bounded bimodule homomorphisms $\widetilde{\Omega}^{1}(A) \rightarrow M$ and bounded $V$-algebra homomorphisms $A \rightarrow A \oplus M$ that split the extension $M \nrightarrow A \oplus M \rightarrow A$.

Proof. Any bounded linear section $s: A \rightarrow A \oplus M$ has the form $a \mapsto(a, \partial(m))$ for a bounded linear map $\partial: A \rightarrow M$. And $s$ is multiplicative if and only if $\partial$ is a derivation. Bounded bimodule maps $\bar{\Omega}^{1}(A) \rightarrow M$ are in bijection with bounded derivations.

We shall also apply the definition and the lemma above to incomplete bornological algebras, where we define $\Omega^{1}(A)$ by leaving out the completions in the construction above. And we shall use a variant of $\Omega^{1}(A)$ for projective systems of algebras. In general, the definition and the lemma above carry over to algebras in any additive monoidal category.

2.6. Tensor algebras and noncommutative differential forms. We describe the tensor algebra of a bornological $V$-module and the algebra of differential forms over a bornological algebra and relate the two. All this goes back to Cuntz and Quillen 16. Their constructions make sense in any additive monoidal category with countable direct sums, and we specialise this generalisation of their constructions to bornological $V$-modules and to complete bornological $V$-modules. We shall mainly use the uncomplete versions below because we are going to modify tensor algebras further before completing them.

Let $W$ be a bornological $V$-module. Equip $W^{\otimes n}$ for $n \geq 1$ with the tensor product bornology and $\mathrm{T} W:=\bigoplus_{n \geq 1} W^{\otimes n}$ with the direct sum bornology; that is, a subset $M$ of $\mathrm{T} W$ is bounded if and only if it is contained in the image of 
$\oplus_{j=1}^{n} N^{\otimes j}$ for some $n \geq 1$ and some bounded submodule $N \subseteq W$. The multiplication $\mathrm{T} W \times \mathrm{T} W \rightarrow \mathrm{T} W$ defined by

$$
\left(x_{1} \otimes \cdots \otimes x_{n}\right) \cdot\left(x_{n+1} \otimes \cdots \otimes x_{n+m}\right):=x_{1} \otimes \cdots \otimes x_{n+m}
$$

makes $\mathrm{T} W$ a bornological algebra, called the tensor algebra of $W$. Let $\sigma_{W}: W \rightarrow \mathrm{T} W$ be the inclusion of the first summand. It is a bounded $V$-module homomorphism, but not an algebra homomorphism.

Lemma 2.6.1. The map $\sigma_{W}: W \rightarrow \mathrm{T} W$ is the universal bounded $V$-module map from $W$ to a bornological algebra. That is, TW is a bornological $V$-algebra and if $f: W \rightarrow S$ is a bounded $V$-module map to a bornological $V$-algebra $S$, then there is a unique bounded algebra homomorphism $f^{\#}: \mathrm{TW} \rightarrow S$ with $f^{\#} \circ \sigma_{W}=f$.

Proof. The multiplication above is well defined and bounded by the universal property of the bornological tensor product. Let $f: W \rightarrow S$ be a bounded $V$-module map. Then there is a unique bounded $V$-module map $f^{\#}: \mathrm{T} W \rightarrow S$ with

$$
f^{\#}\left(x_{1} \otimes \cdots \otimes x_{n}\right):=f\left(x_{1}\right) \cdots f\left(x_{n}\right)
$$

for all $x_{1}, \ldots, x_{n} \in W$. This is a bounded algebra homomorphism. And it is the unique one with $f^{\#} \circ \sigma_{W}=f$.

Let $W$ be a complete bornological $V$-module. The completion of $\mathrm{T} W$ is

$$
\text { T } W:=\bigoplus_{n \geq 1} W^{\varpi^{\circledR} n},
$$

the direct sum of the completed tensor products, equipped with the direct sum bornology. By the universal property of completions, the canonical arrow $\widetilde{\sigma_{W}}: W \rightarrow$ T $W$ is the universal bounded $V$-module map from $W$ to a complete bornological algebra. That is, Т $W$ is a complete bornological $V$-algebra and if $f: W \rightarrow S$ is a bounded $V$-module map to a complete bornological $V$-algebra $S$, then there is a unique bounded algebra homomorphism $f^{\#}:$ T $W \rightarrow S$ with $f^{\#} \circ \overline{\sigma_{W}}=f$.

REMARK 2.6.2. If $W$ is torsion-free, then so is TW. If $W$ is complete and torsion-free, then so is T $W$. This uses [27. Theorem 4.6 and Proposition 4.12] and that completeness and torsion-freeness are hereditary for direct sums.

Let $R$ be a bornological $V$-algebra. Then so is TR. The identity map on $R$ induces a bounded homomorphism $p:=\mathrm{id}_{R}^{\#}: \mathrm{T} R \rightarrow R$ by Lemma 2.0.2. Let

$$
\mathrm{J} R:=\operatorname{ker}(p: \mathrm{T} R \rightarrow R) .
$$

This is a closed two-sided ideal in $\mathrm{T} R$. The inclusion $\mathrm{J} R \rightarrow \mathrm{T} R$ and the projection $p: \mathrm{T} R \rightarrow R$ form an extension of bornological $V$-algebras, which splits by the bounded $V$-module map $\sigma_{R}: R \rightarrow \mathrm{T} R$. Similarly, if $R$ is a complete bornological $V$-algebra, then there is an extension of complete bornological $V$-algebras

$$
\mathrm{J} R \nrightarrow \mathrm{T} R \rightarrow R
$$

that splits by the bounded $V$-module map $\overline{\sigma_{R}}$.

The unitalisation of $R$ is $R^{+}:=R \oplus V$ with the multiplication

$$
(x, \lambda) \cdot(y, \mu):=(x y+\mu x+\lambda y, \lambda \mu)
$$

for $x, y \in R, \lambda, \mu \in V$. So $(0,1)$ is the unit element in $R^{+}$, which we denote simply by 1 . The inclusion map $R \rightarrow R^{+}$is the universal bounded homomorphism from $R$ to a unital bornological algebra. 
We are going to rewrite the tensor algebra using the Fedosov product on the algebra of noncommutative differential forms, following Cuntz and Quillen 16]. This alternative picture is important because it allows to describe the ideal $J R$ and the tube algebras that we shall need. It is sketched in [26. Appendix A.3-4] why all this continues to work for algebras in symmetric monoidal categories. This observation goes back further to $\mathbf{1 5}$.

Let $\Omega^{0} R:=R$ and, for $n \geq 1$, let $\Omega^{n} R:=R^{+} \otimes R^{\otimes n}$, equipped with the tensor product bornology. That is, a submodule $N \subseteq \Omega^{n} R$ is bounded if and only if there is a bounded submodule $M \subseteq R$ such that $N$ is contained in the image of $\Omega^{n} M=M^{+} \otimes M^{\otimes n}$. Let $\Omega R:=\bigoplus_{n \geq 0} \Omega^{n} R$, equipped with the direct sum bornology. We interpret an element $x_{0} \otimes x_{1} \otimes \cdots \otimes x_{n} \in \Omega^{n} R$ as a noncommutative differential form $x_{0} \mathrm{~d} x_{1} \ldots \mathrm{d} x_{n}$. There is a unique structure of differential graded algebra on $\Omega R$ whose multiplication restricts to the given multiplication on $R=\Omega^{0} R$ and whose differential satisfies

$$
\mathrm{d}\left(x_{0} \mathrm{~d} x_{1} \ldots \mathrm{d} x_{n}\right):=1 \cdot \mathrm{d} x_{0} \mathrm{~d} x_{1} \ldots \mathrm{d} x_{n} .
$$

Namely, the (graded) Leibniz rule dictates that

$$
x_{0} \mathrm{~d} x_{1} \ldots \mathrm{d} x_{n} \cdot x_{n+1} \mathrm{~d} x_{n+2} \ldots \mathrm{d} x_{n+m}:=\sum_{j=0}^{n}(-1)^{n-j} x_{0} \mathrm{~d} x_{1} \ldots \mathrm{d}\left(x_{j} \cdot x_{j+1}\right) \ldots \mathrm{d} x_{n+m} .
$$

The Fedosov product on a differential graded algebra such as $\Omega R$ is defined by

$$
\xi \odot \eta:=\xi \eta-(-1)^{i \cdot j} d(\xi) d(\eta) \quad \text { for } \xi \in \Omega^{i} R, \eta \in \Omega^{j} R .
$$

Recall the notation $M^{(n)}:=\sum_{i=1}^{n} M^{i}$. If $p, q \geq 0$ and $M, N \subseteq R$ are bounded $V$-submodules, then

$$
\Omega^{p} M \odot \Omega^{q} N \subseteq \Omega^{p+q}\left((M+N)^{(2)}\right) \oplus \Omega^{p+q+2}((M+N)) .
$$

Hence $(\Omega R, \odot)$ is a bornological algebra. Its completion $\bar{\Omega} R$ is the bornological direct sum $\oplus_{n \geq 0} \bar{\Omega}^{n} R$ of the completed differential forms. Let $\Omega^{\mathrm{ev}} R \subseteq \Omega R$ be the bornological subalgebra of differential forms of even degree. In the following, we always equip $\Omega^{\mathrm{ev}} R$ with the Fedosov product.

The inclusion map $R=\Omega^{0} R \rightarrow \Omega^{\mathrm{ev}} R$ induces a bounded homomorphism

$$
\mathrm{T} R \rightarrow \Omega^{\mathrm{ev}} R, \quad x_{1} \otimes \cdots \otimes x_{n} \mapsto x_{1} \odot \cdots \odot x_{n},
$$

by Lemma 2.0.2 which is, in fact, a bornological isomorphism. To understand why, let $f: R \rightarrow S$ be a $V$-module map. Its curvature is the $V$-module map

$$
\omega_{f}: R \otimes R \rightarrow S, \quad \omega_{f}(x, y)=f(x \cdot y)-f(x) \cdot f(y) .
$$

It is bounded if $f$ is. The composite of the induced homomorphism $f^{\#}: \mathrm{T} R \rightarrow S$ with the inverse of the map in 2.6.6 must be given by the formula

$$
f^{\#}\left(x_{0} \mathrm{~d} x_{1} \ldots \mathrm{d} x_{2 n}\right)=f\left(x_{0}\right) \cdot \omega_{f}\left(x_{1}, x_{2}\right) \cdots \omega_{f}\left(x_{2 n-1}, x_{2 n}\right)
$$

because the inclusion map $R \rightarrow \Omega^{\mathrm{ev}} R$ has the curvature $(x, y) \mapsto x \cdot y-x \odot y=\mathrm{d} x \mathrm{~d} y$. Indeed, this defines a bounded homomorphism $f^{\#}: \Omega^{\mathrm{ev}} R \rightarrow S$. So $\Omega^{\mathrm{ev}} R$ enjoys the same universal property as $\mathrm{T} R$. Then the map in 2.6.6 is a bornological isomorphism.

The map $p: \mathrm{T} R \rightarrow R$ corresponds to the map $p: \Omega^{\mathrm{ev}} R \rightarrow R$ that vanishes on $\Omega^{2 n} R$ for $n \geq 1$ and is the identity on $\Omega^{0} R=R$. Therefore, the isomorphism $\mathrm{T} R \cong \Omega^{\mathrm{ev}} R$ maps $J R$ onto $\oplus_{n \geq 1} \Omega^{2 n} R$. Then it follows by induction that the isomorphism maps the ideal $\mathrm{J} R^{m}$ onto $\oplus_{n \geq m} \Omega^{2 n} R$. This simple description of all the powers $\mathrm{J} R^{m}$ is 
the main point of rewriting the tensor algebra using the Fedosov product on the even-degree differential forms.

REMARK 2.6.8. The map $\mathrm{J} R^{\otimes m} \rightarrow \mathrm{J} R^{m}$ splits by the bounded $V$-module map given by

$a_{0} \mathrm{~d} a_{1} \ldots \mathrm{d} a_{2(m+n)} \mapsto a_{0} \mathrm{~d} a_{1} \mathrm{~d} a_{2} \otimes \mathrm{d} a_{3} \mathrm{~d} a_{4} \otimes \cdots \otimes \mathrm{d} a_{2 m-3} \mathrm{~d} a_{2 m-2} \otimes \mathrm{d} a_{2 m-1} \ldots \mathrm{d} a_{2 n}$.

Thus $\mathrm{J} R^{\otimes m} \rightarrow \mathrm{J} R^{m}$ is a quotient map, and the same is true upon completion.

2.7. The X-complex. The $X$-complex introduced by Cuntz and Quillen in $\mathbf{1 7}$ is an important ingredient in their approach to cyclic homology theories. It is defined for algebras in additive monoidal categories (see also [26. Appendix A.6]). We shall specialise this definition to the additive monoidal category of complete bornological algebras over $F$ or $V$.

Let $\bar{\Omega}^{1}(S) /\left[\right.$, ] be the commutator quotient of $\widetilde{\Omega}^{1}(S)$, that is, the quotient of $\Omega^{1}(S)$ by the closure of the image of

$$
S \Phi \bar{\Omega}^{1}(S) \rightarrow \bar{\Omega}^{1}(S), \quad x \otimes \omega \mapsto x \cdot \omega-\omega \cdot x .
$$

With the quotient bornology, this is a complete bornological $V$-module (see $\mathbf{2 7}$, Theorem 2.3]). The closure comes in because we take a cokernel in the category of complete bornological $V$-modules, which forces us to make the quotient separated.

Let $q: \widetilde{\Omega}^{1}(S) \rightarrow \widetilde{\Omega}^{1}(S) /[$,$] be the quotient map. There is a unique bounded$ linear map $b: \Omega^{1}(S) \rightarrow S$ that satisfies $b(x \mathrm{~d} y)=x \cdot y-y \cdot x$. It descends to a bounded linear map $\tilde{b}: \Omega^{1}(S) /[$, ] $\rightarrow S$. The $X$-complex of $S$ is the following $\mathbb{Z} / 2$-graded chain complex of complete bornological $V$-modules:

$$
X(S):=\left(S \underset{\tilde{b}}{\stackrel{q \circ \mathrm{d}}{\rightleftarrows}} \Omega^{1}(S) /[\cdot, \cdot]\right) .
$$

We briefly call $\mathbb{Z} / 2$-graded chain complexes supercomplexes. If $S$ is a complete bornological $F$-algebra, then $X(S)$ is even a supercomplex of complete bornological $F$-vector spaces.

\section{Definition of analytic cyclic homology}

Let $A$ be a torsion-free, complete bornological $V$-algebra. We are going to define the analytic cyclic homology of $A$. The idea is to make a universal "analytically nilpotent" extension of $A$ and then take the X-complex of that, tensored with $F$ to ensure its homotopy invariance. (The conept of analytic nilpotence will be introduced later in Section 4.3) The starting point is the universal extension with a bounded linear section, which is given by the tensor algebra extension. To make the kernel of this extension nilpotent $\bmod \pi$, we pass to a tube algebra. Then we dagger complete this kernel to make it analytically nilpotent. The tube algebra construction produces a projective system of algebras. Tensoring with $F$ and taking the X-complex, we thus get a projective systems of chain complexes. We could define analytic cyclic homology as an invariant in a suitable derived category of such chain complexes. Our main theorems hold in that setting. We prefer, however, to define it as an ordinary $F$-vector space. Therefore, we also apply the homotopy projective limit and take homology in the very end.

Now we go through the construction in small steps. In the first step, let

$$
R:=\mathrm{T} A, \quad I:=\mathrm{J} A,
$$


be the tensor algebra over $A$ and the kernel of the canonical homomorphism $\mathrm{T} A \rightarrow A$.

The second step enlarges $R$ to a projective system of tube algebras relative to powers of the ideal $I$ :

DeFinition 3.1. Let $R$ be a torsion-free bornological $V$-algebra and $I$ an ideal in $R$. Let $I^{j}$ for $j \in \mathbb{N}^{*}$ denote the $V$-linear span of products $x_{1} \cdots x_{j}$ with $x_{1}, \ldots, x_{j} \in I$. The tube algebra of $I^{l} \triangleleft R$ for $l \in \mathbb{N}^{*}$ is

$$
\mathcal{U}\left(R, I^{l}\right):=\sum_{j=0}^{\infty} \pi^{-j} I^{l \cdot j} \subseteq R \otimes F
$$

with the subspace bornology; this is indeed a $V$-subalgebra of $R \otimes F$. If $l \geq j$, then $\mathcal{U}\left(R, I^{l}\right) \subseteq \mathcal{U}\left(R, I^{j}\right)$ is a bornological subalgebra. Let $\mathcal{U}\left(R, I^{\infty}\right)$ be the projective system of bornological $V$-algebras $\left(\mathcal{U}\left(R, I^{l}\right)\right)_{l \in \mathbb{N}^{*}}$.

Since $\mathcal{U}\left(R, I^{l}\right)$ is defined as a bornological submodule of an $F$-vector space, it is bornologically torsion-free. And the inclusion $R \hookrightarrow \mathcal{U}\left(R, I^{l}\right)$ induces a bornological isomorphism $\mathcal{U}\left(R, I^{l}\right) \otimes F \cong R \otimes F$.

Remark 3.2. In [11. Definition 3.1.19], the tube algebra $\mathcal{U}\left(R, I^{l}\right)$ of a bornological $V$-algebra is equipped with a different bornology, namely, the bornology that is generated by subsets bounded in $R$ and subsets of the form $\pi^{-1} M^{l}$ for bounded subsets $M \subseteq I$. This makes no difference if $R$ carries the fine bornology. For general $R$, however, the two bornologies on the tube algebra need not be the same. It is easy to check that both bornologies induce the same bornology on $\mathcal{U}\left(R, I^{l}\right) \otimes F \cong R \otimes F$. Thus the two bornologies coincide if and only if the bornology defined in [11] is bornologically torsion-free. This concept is introduced only later in $\mathbf{2 7}$. The more complicated bornology defined in [11 gives the tube algebra the expected universal property for bornological algebras that are torsion-free as algebras, but not bornologically torsion-free.

The third step equips $\mathcal{U}\left(R, I^{l}\right)$ for $l \in \mathbb{N}^{*}$ with the linear growth bornology relative to the ideal $\mathcal{U}\left(I, I^{l}\right)$. This gives a projective system of bornological algebras

$$
\mathcal{U}\left(R, I^{\infty}\right)_{\lg \left(\mathcal{U}\left(I, I^{\infty}\right)\right)}=\left(\mathcal{U}\left(R, I^{l}\right)_{\lg \left(\mathcal{U}\left(I, I^{l}\right)\right)}\right)_{l \in \mathbb{N}^{*}}
$$

because the inclusion homomorphism $\mathcal{U}\left(R, I^{l+1}\right) \hookrightarrow \mathcal{U}\left(R, I^{l}\right)$ maps $\mathcal{U}\left(I, I^{l+1}\right)$ to $\mathcal{U}\left(I, I^{l}\right)$. All these bornological algebras are torsion-free by Lemma 2.2.7

The fourth step applies the completion functor. By [27. Theorem 4.6], this gives a projective system of complete, torsion-free bornological $V$-algebras

$$
\left(\mathcal{U}\left(R, I^{\infty}\right), \mathcal{U}\left(I, I^{\infty}\right)\right)^{\dagger}=\left(\left(\mathcal{U}\left(R, I^{l}\right), \mathcal{U}\left(I, I^{l}\right)\right)^{\dagger}\right)_{l \in \mathbb{N}^{*}} .
$$

The fifth step is to tensor with $F$. This gives a projective system of complete bornological $F$-algebras

$$
\left(\mathcal{U}\left(R, I^{\infty}\right), \mathcal{U}\left(I, I^{\infty}\right)\right)^{\dagger} \otimes F:=\left(\left(\mathcal{U}\left(R, I^{l}\right), \mathcal{U}\left(I, I^{l}\right)\right)^{\dagger} \otimes F\right)_{l \in \mathbb{N}^{*}}
$$

The sixth step is to take the $X$-complex. Being natural, it extends to a functor from projective systems of complete bornological algebras to projective systems of supercomplexes. In particular, the canonical maps $\mathcal{U}\left(R, I^{l+1}\right) \rightarrow \mathcal{U}\left(R, I^{l}\right)$ induce bounded chain maps

$$
\sigma_{l}: X\left(\left(\mathcal{U}\left(R, I^{l+1}\right), \mathcal{U}\left(I, I^{l+1}\right)\right)^{\dagger} \otimes F\right) \rightarrow X\left(\left(\mathcal{U}\left(R, I^{l}\right), \mathcal{U}\left(I, I^{l}\right)\right)^{\dagger} \otimes F\right) .
$$


These define a projective system of supercomplexes of complete bornological $F$-vector spaces, which we denote by

$$
\mathbb{H} \mathbb{A}(A):=X\left(\left(\mathcal{U}\left(R, I^{\infty}\right), \mathcal{U}\left(I, I^{\infty}\right)\right)^{\dagger} \otimes F\right)
$$

The seventh step takes the homotopy projective limit holim $\mathbb{H} \mathbb{A}(A)$. More explicitly, this is the mapping cone of the chain map

$$
\begin{aligned}
\prod_{l \in \mathbb{N}^{*}} X\left(\left(\mathcal{U}\left(R, I^{l}\right), \mathcal{U}\left(I, I^{l}\right)\right)^{\dagger} \otimes F\right) & \rightarrow \prod_{l \in \mathbb{N}^{*}} X\left(\left(\mathcal{U}\left(R, I^{l}\right), \mathcal{U}\left(I, I^{l}\right)\right)^{\dagger} \otimes F\right), \\
\left(x_{l}\right) & \mapsto\left(x_{l}-\sigma_{l}\left(x_{l+1}\right)\right)_{l \in \mathbb{N}^{*}} .
\end{aligned}
$$

It is a supercomplex of complete bornological $F$-vector spaces. The final, eighth step takes its homology:

Definition 3.3. The analytic cyclic homology $\operatorname{HA}_{*}(A)$ of a complete, torsionfree bornological -algebra $A$ for $* \in \mathbb{Z} / 2$ is the homology of holim $\mathbb{H} \mathbb{A}(A)$, that is, the quotient of the kernel of the differential by the image of the differential. We do not take the closure of the image, so that this quotient need not be bornologically separated. For this reason, we prefer to forget the induced bornology on $\mathrm{HA}_{*}(A)$.

3.1. Bivariant analytic cyclic homology. Besides the analytic cyclic homology functor $\mathrm{HA}_{*}$, we also have the functor $\mathbb{H} \mathbb{A}$ taking values in suitable homotopy categories of chain complexes of projective systems of bornological $V$-modules. This functor contains more information. In particular, it yields a bivariant analytic cyclic homology theory by letting $\mathrm{HA}_{*}\left(A_{1}, A_{2}\right)$ be the set of morphisms $\mathbb{H} \mathbb{A}\left(A_{1}\right) \rightarrow \mathbb{H} \mathbb{A}\left(A_{2}\right)$. Cuntz and Quillen use the same idea in $\mathbf{1 7}$ to extend periodic cyclic homology to a bivariant theory. The actual definition of $\overrightarrow{\mathrm{HA}}_{*}\left(A_{1}, A_{2}\right)$ depends on the choice of the target category, however, and this is somewhat flexible. We do not pick any choice in this article, but only point out two natural options.

The analytic cyclic homology computations in this paper often prove a chain homotopy equivalence $\mathbb{H} \mathbb{A}(A) \simeq \mathbb{H} \mathbb{A}(B)$, as supercomplexes of projective systems of bornological $V$-modules. These are equivalences in the homotopy category of supercomplexes, where homotopy is understood simply as chain homotopy. In all cases where we compute $\mathrm{HA}_{*}(A)$ in this paper, we actually prove that $\mathbb{H} \mathbb{A}(A)$ is chain homotopy equivalent to a supercomplex with zero boundary map, so that it contains no more information than the bornological $F$-vector space $\mathrm{HA}_{*}(A)$. Homotopy projective limits are sufficiently compatible with chain homotopies to preserve chain homotopy equivalence; and this implies an isomorphism on homology.

A larger class of weak equivalences is used in $\mathbf{1 5}$ to define a homotopy category of chain complexes of projective systems. A good aspect of this construction is that it clarifies the role of the homotopy projective limit: this just replaces a given complex by one that is weakly equivalent to it and fibrant in a suitable sense, so that the arrows to it in the homotopy category are the same as chain homotopy classes of chain maps. Thus $\mathrm{HA}_{*}(A)$ is isomorphic to the space of arrows from the trivial supercomplex $V$ to $\mathbb{H} \mathbb{A}(A)$ in the homotopy category of $\mathbf{1 5}$. We will see later that $\mathbb{H} \mathbb{A}(V)$ is chain homotopy equivalent to the trivial supercomplex $V$ (see Corollary 4.7.3). So the homotopy category of $\mathbf{1 5}$ is such that the bivariant analytic cyclic homology group $\mathrm{HA}_{*}(V, A)$ simplifies to $\mathrm{HA}_{*}(A)$. 


\section{Analytic nilpotence and analytically quasi-free resolutions}

Cuntz and Quillen described the periodic cyclic homology of an algebra $A$ as the homology of the $X$-complex of a certain projective system built from the tensor algebra T $A$ of $A$. This approach to periodic cyclic homology is the key to proving that it satisfies excision. The Cuntz-Quillen approach is carried over to more analytic versions of periodic cyclic homology in [26]. Our proof of excision for $\mathrm{HA}_{*}$ in Section 5 will follow the pattern in $\mathbf{2 6}$. In this section, we explain how $\mathrm{HA}_{*}$ as defined above fits into this framework.

4.1. Pro-Algebras. An important idea in $[\mathbf{2 6}]$ is that an analytic variant of periodic cyclic homology is defined by a suitable notion of "analytic nilpotence". This leads to an analytic tensor algebra of an algebra $A$, which is universal among analytically nilpotent extensions of $A$. It also leads to the concept of analytically quasi-free algebras. The theory is set up so that any two analytically quasi-free, analytically nilpotent extensions of a given algebra are homotopy equivalent. In characteristic 0 , this implies that their $X$-complexes are chain homotopy equivalent. Thus the $X$-complex of the analytic tensor algebra is chain homotopy equivalent to the $X$-complex of any analytically quasi-free resolution of $A$. In this discussion, "algebras" are always more complex objects - such as projective systems of algebras or bornological algebras - because there is no suitable concept of analytic nilpotence for mere algebras without extra structure. For the analytic cyclic homology defined above, the appropriate type of algebra is a projective system of torsion-free, complete bornological $V$-algebras. For brevity, we call torsion-free, complete bornological $V$-algebras algebras and projective systems of them pro-algebras.

A pro-algebra is given by a directed set $(N, \leq)$, algebras $A_{n}$ for $n \in N$, and bounded algebra homomorphisms $\alpha_{m, n}: A_{n} \rightarrow A_{m}$ for $m, n \in N$ with $n \geq m$ that satisfy $\alpha_{m, m}=\operatorname{id}_{A_{m}}$ for all $m \in N$ and $\alpha_{m, n} \circ \alpha_{n, p}=\alpha_{m, p}$ for all $m, n, p \in N$ with $p \geq n \geq m$. The morphism set between two pro-algebras is

$$
\operatorname{Hom}\left(\left(A_{l}\right)_{l \in L},\left(B_{n}\right)_{n \in N}\right):={\underset{\lim }{n}}_{l} \underset{l}{\lim } \operatorname{Hom}\left(A_{l}, B_{n}\right) \text {. }
$$

We shall only need pro-algebras $\left(A_{n}\right)_{n \in N}$ where $N$ is countable. Restricting to a cofinal increasing sequence in $N$ gives an isomorphic pro-algebra with $N=\mathbb{N}$. Then the maps $\alpha_{m, n}$ are uniquely determined by $\alpha_{n, n+1}: A_{n+1} \rightarrow A_{n}$ for $n \in \mathbb{N}$.

An algebra $A$ is also a pro-algebra by taking $A_{n}=A$ and $\alpha_{n, n+1}:=\mathrm{id}_{A}$ for all $n \in \mathbb{N}$. Such projective systems are called constant. For a pro-algebra $A=\left(A_{n}, \alpha_{m, n}\right)$, there are canonical morphisms $A \rightarrow \operatorname{const}\left(A_{n}\right)$ for all $n \in N$.

The analytic tensor algebra of a torsion-free algebra $A$ is the torsion-free pro-algebra $\left(\mathcal{U}\left(\mathrm{T} A, \mathrm{~J} A^{\infty}\right), \mathcal{U}\left(\mathrm{J} A, \mathrm{~J} A^{\infty}\right)\right)^{\dagger}$ in the above definition of analytic cyclic homology. This comes with a canonical homomorphism to $A$, whose kernel is the proalgebra $\left(\mathcal{U}\left(\mathrm{J} A, \mathrm{~J} A^{\infty}\right)\right)^{\dagger}$. This projective system of complete, torsion-free bornological algebras has two important extra properties: it is semi-dagger - hence dagger - and nilpotent $\bmod \pi$ - this concept will be defined below. A pro-algebra with these two properties is called analytically nilpotent. The tube algebra construction and the relative dagger completion in the construction of the analytic tensor algebra are the universal way to make a pro-algebra extension with an analytically nilpotent kernel.

Any functor from algebras to algebras extends canonically to an endofunctor on the category of pro-algebras by applying it entrywise. The definition of analytic cyclic homology already used this extension to pro-algebras for completions and 
tensor products with $F$. The constructions of $\mathrm{T} A$ and $\mathrm{J} A$ for algebras are also functors and thus extend to pro-algebras. So is the tensor product bifunctor $-\bar{\otimes}-$, which extends to pro-algebras by

$$
\begin{aligned}
\left(A_{n}, \alpha_{m, n}\right)_{m, n \in N} \otimes\left(B_{n}, \beta_{m, n}\right)_{m, n \in N^{\prime}} & \\
:= & \left(A_{n_{1}} \otimes B_{n_{2}}, \alpha_{m_{1}, n_{1}} \otimes \beta_{m_{2}, n_{2}}\right)_{m_{1}, n_{1} \in N, m_{2}, n_{2} \in N^{\prime}} .
\end{aligned}
$$

In particular, we may tensor a pro-algebra with an algebra such as $V[t]^{\dagger}$, viewed as a constant pro-algebra.

DeFinition 4.1.1. An elementary dagger homotopy between two morphisms of pro-algebras $f_{0}, f_{1}: A \rightrightarrows B$ is a morphism of pro-algebras $f: A \rightarrow B \Phi V[t]^{\dagger}$ that satisfies $\left(\mathrm{id}_{A} \otimes \mathrm{ev}_{t}\right) \circ f=f_{t}$ for $t=0,1$. We call $f_{0}, f_{1}$ elementary dagger homotopic if there is such a homotopy. Dagger homotopy is the equivalence relation generated by elementary dagger homotopy.

4.2. The universal property of the tube algebra construction. First, we generalise the construction of tube algebras to pro-algebras. Actually, in this subsection, we drop the completeness assumption for algebras because tube algebras are usually incomplete. So "algebras" are torsion-free bornological algebras and pro-algebras are projective systems of such algebras until the end of this subsection.

An ideal in a pro-algebra $A=\left(A_{n}, \alpha_{m, n}\right)_{i \in N}$ is a family of ideals $I_{n} \triangleleft A_{n}$ with $\alpha_{m, n}\left(I_{n}\right) \subseteq I_{m}$ for all $n, m \in N$ with $n \geq m$; then $\alpha_{m, n}$ induces homomorphisms $\mathcal{U}\left(A_{n}, I_{n}^{l}\right) \rightarrow \mathcal{U}\left(A_{m}, I_{m}^{l}\right)$ for all $l \in \mathbb{N}^{*}$, which intertwine the inclusion maps $\mathcal{U}\left(A_{n}, I_{n}^{l}\right) \hookrightarrow \mathcal{U}\left(A_{n}, I_{n}^{j}\right)$ for $l \geq j$. These homomorphisms form a pro-algebra

$$
\mathcal{U}\left(A, I^{\infty}\right):=\left(\mathcal{U}\left(A_{n}, I_{n}^{l}\right)\right)_{n \in N, l \in \mathbb{N}^{*}} .
$$

If $l \in \mathbb{N}^{*}$, then $\mathcal{U}\left(A, I^{l}\right):=\left(\mathcal{U}\left(A_{n}, I_{n}^{l}\right)\right)_{n \in N}$ is a pro-algebra. The pro-algebra $\mathcal{U}\left(A, I^{l}\right)$ for $l \in \mathbb{N}^{*} \cup\{\infty\}$ contains $\mathcal{U}\left(I, I^{l}\right)$ as an ideal. Since $A_{n} \subseteq \mathcal{U}\left(A_{n}, I_{n}^{l}\right)$ for all $n \in N$, $l \in \mathbb{N}^{*}$, the inclusion maps define a pro-algebra homomorphism $\iota_{A, I}: A \rightarrow \mathcal{U}\left(A, I^{\infty}\right)$.

REMARK 4.2.1. The notion of ideal above suffices for our purposes and is convenient to define the tube algebra quickly. It has the problem of not being invariant under isomorphism of pro-algebras. A better definition would be to define an ideal to be the kernel of a pro-algebra homomorphism. It is, however, possible to switch to isomorphic pro-algebras to make a pro-algebra homomorphism into a homomorphism of diagrams. And then the kernel becomes a family of ideals as above. This allows to extend the construction of tube algebras to ideals in the more general sense.

Definition 4.2.2. A pro-algebra $\left(A_{n}, \alpha_{m, n}\right)_{n \in N}$ is nilpotent $\bmod \pi$ if, for each $m \in N$, there are $n \in N_{\geq m}$ and $l \in \mathbb{N}^{*}$ such that $\alpha_{m, n}\left(A_{n}^{l}\right) \subseteq \pi A_{m}$; here $A_{n}^{l}$ denotes the $V$-submodule generated by all products $x_{1} \cdots x_{l}$ of $l$ factors in $A_{n}$.

REMARK 4.2.3. Let $A=\left(A_{n}, \alpha_{m, n}\right)_{m, n \in N}$ be a pro-algebra. Let $A /(\pi)$ be the projective system of $\mathbb{F}$-algebras formed by the quotients $A_{n} /(\pi)$ with the homomorphisms induced by $\alpha_{m, n}$. By definition, $A$ is nilpotent $\bmod \pi$ if and only if $A /(\pi)$ has the following property: for each $n \in N$ there are $m \in N$ and $l \in \mathbb{N}^{*}$ such that the $l$-fold multiplication map $\left(A_{m} /(\pi)\right)^{\otimes l} \rightarrow A_{n} /(\pi)$ is zero. This is equivalent to the definition that a projective system of $\mathbb{F}$-algebras is pro-nilpotent in 26. Definition 4.3]. 
Proposition 4.2.4. Let $A$ and $B$ be pro-algebras and let $I$ and $J$ be ideals in $A$ and $B$, respectively. Let $\varphi: A \rightarrow B$ be a pro-algebra morphism that restricts to a pro-algebra morphism $I \rightarrow J$. Let $\iota_{A, I}: A \rightarrow \mathcal{U}\left(A, I^{\infty}\right)$ denote the canonical pro-algebra morphism.

(1) The pro-algebra $\mathcal{U}\left(I, I^{\infty}\right)$ is nilpotent $\bmod \pi$.

(2) If $J$ is nilpotent mod $\pi$, then there is a unique morphism $\bar{\varphi}: \mathcal{U}\left(A, I^{\infty}\right) \rightarrow B$ with $\bar{\varphi} \circ \iota_{A, I}=\varphi$. It restricts to a morphism $\mathcal{U}\left(I, I^{\infty}\right) \rightarrow J$.

(3) There is a unique morphism $\varphi_{*}: \mathcal{U}\left(A, I^{\infty}\right) \rightarrow \mathcal{U}\left(B, J^{\infty}\right)$ with $\varphi_{*} \circ \iota_{A, I}=$ $\iota_{B, J} \circ \varphi$. It restricts to a morphism $\mathcal{U}\left(I, I^{\infty}\right) \rightarrow \mathcal{U}\left(J, J^{\infty}\right)$.

Proof. Write $A=\left(A_{n}, \alpha_{m, n}\right)_{n \in N}, I=\left(I_{n}\right)_{n \in N}$ with ideals $I_{n}$ in $A_{n}$ with $\alpha_{m, n}\left(I_{n}\right) \subseteq I_{m}$ and $B=\left(B_{n}, \beta_{m, n}\right)_{n \in N^{\prime}}, J=\left(J_{n}\right)_{n \in N^{\prime}}$ with ideals $J_{n}$ in $B_{n}$ with $\beta_{m, n}\left(J_{n}\right) \subseteq J_{m}$. The tube algebra $\mathcal{U}\left(A, I^{\infty}\right)$ is the projective limit of the tube algebras $\mathcal{U}\left(A_{n}, I_{n}^{\infty}\right)$ in the category of pro-algebras.

Being nilpotent $\bmod \pi$ is hereditary for projective limits. So it suffices to prove (1) when $A$ is a constant pro-algebra. Fix $n \in \mathbb{N}^{*}$ and let $m=2 n, l=n$. Then

$$
\mathcal{U}\left(I, I^{m}\right)^{l}=\mathcal{U}\left(I, I^{2 n}\right)^{n}=\left(I+\sum_{j=1}^{\infty} \pi^{-j} I^{2 n j}\right)^{n} \subseteq I^{n}+\sum_{j=1}^{\infty} \pi^{-j} I^{2 n j}
$$

because $\sum_{j=1}^{\infty} \pi^{-j} I^{2 n j}$ is an ideal in $\mathcal{U}\left(A, I^{2 n}\right)$. Since $\pi^{-1} I^{n}$ and $\pi^{-2 j} I^{2 n j}$ are contained in $\mathcal{U}\left(I, I^{n}\right)$, all summands on the right hand side of 4.2 .5$)$ are contained in $\pi \cdot \mathcal{U}\left(I, I^{n}\right)$. Thus $\mathcal{U}\left(I, I^{\infty}\right)$ is nilpotent $\bmod \pi$.

We prove statement (2) The morphism $\varphi: A \rightarrow B$ is described by a coherent family of $V$-algebra homomorphisms $\varphi_{n}: A_{\psi(n)} \rightarrow B_{n}$ for all $n \in N^{\prime}$. Each $B_{n}$ is torsion-free by our definition of "algebra". Then the homomorphism $\varphi_{n}$ is determined by $\varphi_{n} \otimes \operatorname{id}_{F}: A_{\psi(n)} \otimes F \rightarrow B_{n} \otimes F$. By construction, $\mathcal{U}\left(A_{\nu}, I^{m}\right) \otimes F=A_{\nu} \otimes F$ for all $\nu \in N, m \in \mathbb{N}^{*}$. Thus a factorisation of $\varphi$ through $\mathcal{U}\left(A, I^{\infty}\right)$ is unique if it exists.

Fix $n \in N^{\prime}$. Since $J$ is nilpotent $\bmod \pi$, there are $m \in N_{\geq n}^{\prime}$ and $l \in \mathbb{N}^{*}$ with $\beta_{n, m}\left(J_{m}^{l}\right) \subseteq \pi \cdot J_{n}$. Since $\varphi$ is coherent, there is $\nu \in N_{\geq \psi(m)}$ with $\beta_{n, m} \circ \varphi_{m} \circ \alpha_{\psi(m), \nu}=$ $\varphi_{n} \circ \alpha_{n, \nu}$. Since $\varphi$ restricts to a morphism $I \rightarrow J$, we may also arrange that $\varphi_{m} \circ \alpha_{\psi(m), \nu}\left(I_{\nu}\right) \subseteq J_{m}$ by increasing $\nu$ if necessary. Hence

$$
\varphi_{n} \circ \alpha_{n, \nu}\left(I_{\nu}^{l}\right)=\beta_{n, m} \circ \varphi_{m} \circ \alpha_{\psi(m), \nu}\left(I_{\nu}^{l}\right) \subseteq \beta_{n, m}\left(J_{m}^{l}\right) \subseteq \pi \cdot J_{n} .
$$

Thus the homomorphism $\left(\varphi_{n} \circ \alpha_{n, \nu}\right) \otimes \operatorname{id}_{F}: A_{\nu} \otimes F \rightarrow B_{n} \otimes F$ maps the tube algebra $\mathcal{U}\left(A_{\nu}, I_{\nu}^{l}\right) \subseteq A_{\nu} \otimes F$ into $B_{n} \subseteq B_{n} \otimes F$ and $\mathcal{U}\left(I_{\nu}, I_{\nu}^{l}\right) \subseteq A_{\nu} \otimes F$ into $J_{n} \subseteq B_{n} \otimes F$. This gives a homomorphism $\bar{\varphi}_{n}: \mathcal{U}\left(A_{\nu}, I_{\nu}^{l}\right) \rightarrow B_{n}$ with $\bar{\varphi}_{n} \circ \iota_{A_{\nu}, I_{\nu}^{l}}=\varphi_{n} \circ \alpha_{n, \nu}$. Since $\mathcal{U}\left(A_{\nu}, I^{m}\right) \subseteq A_{\nu} \otimes F$, the homomorphisms $\bar{\varphi}_{n}$ inherit the coherence property of a pro-algebra morphism from the maps $\varphi_{n}$.

We prove statement (3) of the proposition. We compose $\varphi: A \rightarrow B$ with the canonical map $B \rightarrow \mathcal{U}\left(B, J^{\infty}\right)$ to get a morphism $A \rightarrow \mathcal{U}\left(B, J^{\infty}\right)$. It restricts to a morphism $I \rightarrow J \rightarrow \mathcal{U}\left(J, J^{\infty}\right)$. The ideal $\mathcal{U}\left(J, J^{\infty}\right)$ in $\mathcal{U}\left(B, J^{\infty}\right)$ is nilpotent $\bmod \pi$ by (1) So (2) shows that our morphism extends uniquely to a morphism $\mathcal{U}\left(A, I^{\infty}\right) \rightarrow \mathcal{U}\left(B, J^{\infty}\right)$ that maps $\mathcal{U}\left(I, I^{\infty}\right)$ to $\mathcal{U}\left(J, J^{\infty}\right)$

We summarise the tube algebra construction in category-theoretic language. Let $\mathfrak{P r o}$ be the category whose objects are pairs $(A, I)$, where $A$ is a pro-algebra and $I$ is an ideal in $A$ and whose morphisms are pro-algebra morphisms that restrict to a morphism between the ideals. The pairs $(A, I)$ where $I$ is nilpotent $\bmod \pi$ form a subcategory $\mathfrak{P} \mathfrak{r o} \mathfrak{n}_{\text {nil }}$ in $\mathfrak{P r o}$. The first two statements in Proposition 4.2.4 
say that the canonical arrow $(A, I) \rightarrow\left(\mathcal{U}\left(A, I^{\infty}\right), \mathcal{U}\left(I, I^{\infty}\right)\right)$ is a universal arrow from $(A, I)$ to an object in $\mathfrak{P r o} \mathfrak{r}_{\text {nil }}$. Thus $\mathfrak{P} \mathfrak{r o}$ nil is a reflective subcategory in $\mathfrak{P r o}$ and the reflector acts on objects by $(A, I) \mapsto\left(\mathcal{U}\left(A, I^{\infty}\right), \mathcal{U}\left(I, I^{\infty}\right)\right)$. Its functoriality is Proposition 4.2.4 (3) If $I$ is already nilpotent $\bmod \pi$, then it follows that the identity map on $A$ extends uniquely to an isomorphism of pro-algebras $\mathcal{U}\left(A, I^{\infty}\right) \cong A$.

The inheritance properties of nilpotence $\bmod \pi$ proven in the following proposition are needed by the analytic cyclic homology machinery in $[\mathbf{2 6}]$.

Proposition 4.2.6. The class of nilpotent mod $\pi$ pro-algebras is closed under the following operations:

- Let $A \stackrel{i}{\rightarrow} B \stackrel{p}{\rightarrow} C$ be an extension of pro-algebras. If $A$ and $C$ are nilpotent $\bmod \pi$, then so is $B$, and vice versa.

- A pro-subalgebra $D \subseteq B$ is nilpotent $\bmod \pi$ if $B$ is so and $B / D$ is isomorphic to a projective system of torsion-free bornological $V$-modules.

- Being nilpotent mod $\pi$ is hereditary for projective limits.

- A tensor product $A \otimes B$ is nilpotent $\bmod \pi$ if $A$ or $B$ is nilpotent $\bmod \pi$.

PROOF. Remark 4.2 .3 translates all these statements to statements about the class of pro-nilpotent projective systems of $\mathbb{F}$-algebras. In this way, the statements follow from [26, Theorem 4.4]. We briefly explain direct proofs for the first two claims. The claims about projective limits and tensor products are easy and left to the reader.

As in 26, we may write any extension of pro-algebras $A \stackrel{i}{\rightarrow} B \stackrel{p}{\rightarrow} C$ as a projective system of extensions $A_{n} \stackrel{i_{n}}{\rightarrow} B_{n} \stackrel{p_{n}}{\rightarrow} C_{n}$, with morphisms of extensions

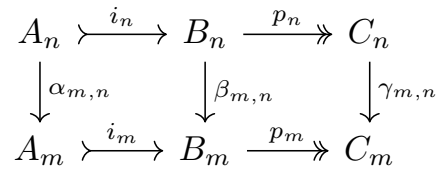

for $n \geq m$ as structure maps (this construction is also explained during the proof of Proposition 4.3 .13 below). Assume that $A$ and $C$ are nilpotent $\bmod \pi$. Pick $m \in N$. There are $n_{1} \in N_{\geq m}$ and $j_{1} \in \mathbb{N}^{*}$ so that $\alpha_{m, n_{1}}\left(A_{n_{1}}^{j_{1}}\right) \subseteq \pi \cdot A_{m}$. And there are $n_{2} \in N_{\geq n_{1}}$ and $j_{2} \in \mathbb{N}^{*}$ so that $\gamma_{n_{1}, n_{2}}\left(C_{n_{2}}^{j_{2}}\right) \subseteq \pi \cdot C_{n_{1}}$. Then $p_{n_{1}}\left(\beta_{n_{1}, n_{2}}\left(B_{n_{2}}^{j_{2}}\right)\right) \subseteq \pi \cdot C_{n_{1}}$. This implies $\beta_{n_{1}, n_{2}}\left(B_{n_{2}}^{j_{2}}\right) \subseteq \pi \cdot B_{n_{1}}+i_{n_{1}}\left(A_{n_{1}}\right)$. Then

$$
\begin{aligned}
& \beta_{m, n_{2}}\left(B_{n_{2}}^{j_{1} \cdot j_{2}}\right) \subseteq \beta_{m, n_{1}}\left(\pi \cdot B_{n_{1}}+i_{n_{1}}\left(A_{n_{1}}\right)\right)^{j_{1}} \subseteq \pi \cdot B_{m}+i_{m}\left(\alpha_{m, n_{1}}\left(A_{n_{1}}^{j_{1}}\right)\right) \\
& \subseteq \pi \cdot B_{m}+i_{m}\left(\pi A_{m}\right) \subseteq \pi \cdot B_{m} .
\end{aligned}
$$

So $B$ is nilpotent $\bmod \pi$. Conversely, if $B$ is nilpotent $\bmod \pi$, then $C$ is nilpotent $\bmod \pi$ because $p_{m}\left(B_{m}\right)=C_{m}$ and $p_{m}\left(\pi \cdot B_{m}\right)=\pi \cdot C_{m}$. The claim that $A$ is nilpotent $\bmod \pi$ if $B$ is follows from the claim about pro-subalgebras.

Given a pro-subalgebra $D \subseteq B$, we may write $B=\left(B_{n}, \beta_{m, n}\right)_{n \in N}$ and $D=$ $\left(D_{n}, \delta_{m, n}\right)_{n \in N}$ so that $D_{n} \subseteq B_{n}$ for all $n \in N$ and $\delta_{m, n}=\left.\beta_{m, n}\right|_{D_{n}}: D_{n} \rightarrow D_{m}$ for all $m, n \in N$ with $m \leq n$. Let $m \in N$. Since $B / D$ is isomorphic to a projective system of torsion-free bornological $V$-modules, there is $n \in N_{\geq m}$ so that the structure map $B_{n} / D_{n} \rightarrow B_{m} / D_{m}$ kills all elements $x \in B_{n} / D_{n}$ with $\pi \cdot x=0$. Equivalently, if $x \in B_{n}$ satisfies $\pi \cdot x \in D_{n}$, then $\beta_{m, n}(x) \in D_{m}$. Thus $\beta_{m, n}\left(\pi \cdot B_{n} \cap D_{n}\right) \subseteq \pi \cdot D_{m}$. If $B$ is nilpotent $\bmod \pi$, then there are $l \in N_{\geq n}$ and $j \in \mathbb{N}^{*}$ with $\beta_{n, l}\left(B_{l}^{j}\right) \subseteq \pi \cdot B_{n}$. Hence

$$
\delta_{m, l}\left(D_{l}^{j}\right) \subseteq \delta_{m, n}\left(\delta_{n, l}\left(D_{l}^{j}\right)\right) \subseteq \beta_{m, n}\left(\pi \cdot B_{n} \cap D_{n}\right) \subseteq \pi \cdot D_{m} .
$$


Thus $D$ is nilpotent $\bmod \pi$.

4.3. Analytically nilpotent pro-algebras. From now on, "algebra" means a complete, torsion-free bornological algebra.

Definition 4.3.1. A pro-algebra $J$ is analytically nilpotent if it is isomorphic to a pro-dagger algebra and nilpotent $\bmod \pi$. It is square-zero if its multiplication map is 0. An extension of pro-algebras $J \rightarrow E \rightarrow A$ is analytically nilpotent or square-zero if $J$ is analytically nilpotent or square-zero, respectively.

In an analytically nilpotent pro-algebra, any power series $\sum c_{n} x^{n}$ for an "element" $x \in J$ and a bounded sequence $\left(c_{n}\right)_{n \in \mathbb{N}}$ in $V$ may be evaluated (see the proof of Proposition 4.3 .6 for the precise meaning of this in a pro-algebra). This uses nilpotence $\bmod \pi$ in order to reduce to sequences whose valuation grows linearly, and being a pro-dagger algebra to ensure that such series converge.

Definition 4.3.2. A pro-linear map between two pro-algebras is a morphism of projective systems of bornological $V$-modules between them; so pro-linear maps need not be multiplicative. An extension of pro-algebras $J \nrightarrow E \rightarrow A$ is semi-split if it splits by a pro-linear map.

Definition 4.3.3. A pro-algebra $A$ is analytically quasi-free if any semi-split analytically nilpotent extension $J \nrightarrow E \rightarrow A$ splits by a pro-algebra homomorphism $A \rightarrow E$. It is quasi-free if any semi-split square-zero extension $J \rightarrow E \rightarrow A$ splits by a pro-algebra homomorphism $A \rightarrow E$.

The following lemma gives an equivalent reformulation of the last definition:

Lemma 4.3.4. A pro-algebra $A$ is analytically quasi-free if and only if, for any semi-split analytically nilpotent extension $J \rightarrow E \rightarrow B$, any homomorphism $f: A \rightarrow B$ lifts to a homomorphism $A \rightarrow E$. A pro-algebra $A$ is quasi-free if and only if, for any semi-split square-zero extension $J \nrightarrow E \rightarrow B$, any homomorphism $f: A \rightarrow B$ lifts to a homomorphism $A \rightarrow E$.

Proof. We may pull the given extension back to a semi-split extension $J \nrightarrow$ $\hat{E} \rightarrow A$, such that a section $A \rightarrow \hat{E}$ is equivalent to a lifting of $f$.

REMARK 4.3.5. A pro-algebra is square-zero if and only if it is isomorphic to a projective system of torsion-free complete bornological $V$-modules, each equipped with the zero map as multiplication. Then it is analytically nilpotent. As a consequence, analytically quasi-free algebras are quasi-free.

Proposition 4.3.6. The base ring $V$ viewed as a constant pro-algebra is analytically quasi-free.

Proof. The proof follows 17, Section 12]. This ideas is, in fact, much older, see 23 . Section 3.6]. Let $J \nrightarrow E \stackrel{p}{\rightarrow} Q$ be a semi-split, analytically nilpotent extension of pro-algebras. Analytic quasi-freeness of $V$ is equivalent to the assertion that any idempotent in $Q$ lifts to an idempotent in $E$. Here by an idempotent in a pro-algebra $A=\left(A_{n}\right)_{n}$, we mean a collection $a=\left(a_{n}\right)_{n}$ of idempotents $a_{n} \in A_{n}$. Each $a_{n} \in A_{n}$ is equivalent to a homomorphism $V \rightarrow A_{n}$.

Let $\dot{e}=\left(\dot{e}_{n}\right)_{n} \in Q$ be an idempotent and let $e \in E$ be the image of $\dot{e}$ under a pro-linear section for $p: E \rightarrow Q$. Let $x:=e-e^{2} \in J$. We use an Ansatz by Cuntz and Quillen to find an idempotent $\hat{e} \in E$ with $e-\hat{e} \in J$. Namely, we assume 
$\hat{e}=e+(2 e-1) \varphi(x)$ for some power series $\varphi \in t \mathbb{Z}[[t]]$. As $J$ is nilpotent $\bmod \pi$, for every $l \in N$, there are $m(l) \geq l$ and $j(l) \in N^{*}$ with $x_{m(l)}^{j(l)}=\pi y_{l}$. To simplify notation, we simply write this as $x^{j}=\pi y$ for some $y \in J$ and $j \in N^{*}$. Finally, since $J$ is also a pro-dagger algebra, $\varphi(x) \in J$ for all $\varphi \in t \mathbb{Z}[[t]]$. We compute

$$
\hat{e}^{2}-\hat{e}=\left(\varphi(x)^{2}+\varphi(x)\right)(1-4 x)-x .
$$

So $\hat{e}^{2}=\hat{e}$ if and only if $\varphi(x)^{2}+\varphi(x)=\frac{x}{1-4 x}$. This is solved by $\varphi(x):=\sum_{n=1}^{\infty}\left(\begin{array}{c}2 n-1 \\ n\end{array}\right) x^{n}$. This defines an element of $J$. Then $\hat{e}$ is the desired idempotent lifting.

Proposition 4.3.7. An algebra $A$ is analytically quasi-free if and only if its unitalisation $A^{+}$is analytically quasi-free.

Proof. Proposition 4.3.6 implies this as in the proof of [26 Proposition 5.53].

Proposition 4.3.8. Let $\left(A_{n}\right)_{n \in \mathbb{N}}$ be a sequence of unital, analytically quasi-free pro-algebras. Then $\bigoplus_{n \in \mathbb{N}} A_{n}$ is analytically quasi-free.

Proof. The proof of [26. Proposition 5.53] carries over to this context.

Corollary 4.3.9. The direct sum $\oplus_{n \in \mathbb{N}} V$ is analytically quasi-free.

Proposition 4.3.10. Let $J_{i} \rightarrow E_{i} \rightarrow A_{i}$ for $i=1,2$ be semi-split, analytically nilpotent extensions of pro-algebras. Assume that $E_{1}$ is analytically quasi-free.

(1) Any pro-algebra morphism $f: A_{1} \rightarrow A_{2}$ lifts to a morphism of extensions

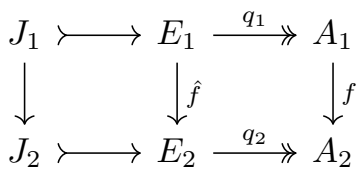

This lifting is unique up to dagger homotopy.

(2) Let $\hat{f}, \hat{g}: E_{1} \rightrightarrows E_{2}$ be pro-algebra homomorphisms that lift homomorphisms $f, g: A_{1} \rightrightarrows A_{2}$. Then an elementary dagger homotopy $h: A_{1} \rightarrow A_{2}{ }^{\otimes} V[t]^{\dagger}$ between $f$ and $g$ lifts to an elementary dagger homotopy $\hat{h}: E_{1} \rightarrow E_{2} \otimes V[t]^{\dagger}$ between $\hat{f}$ and $\hat{g}$.

(3) Any elementary dagger homotopy $A_{1} \rightarrow A_{2} \otimes V[t]^{\dagger}$ lifts to an elementary dagger homotopy $E_{1} \rightarrow E_{2} \bar{\otimes} V[t]^{\dagger}$.

Proof. Let $f: A_{1} \rightarrow A_{2}$ be a pro-algebra homomorphism. Since $E_{1}$ is analytically quasi-free and the extension $J_{2} \rightarrow E_{2} \rightarrow A_{2}$ is semi-split and analytically nilpotent, the homomorphism $f \circ q_{1}$ lifts to a homomorphism $\hat{f}: E_{1} \rightarrow E_{2}$. Since $q_{2} \circ \hat{f}=f \circ q_{1}$ vanishes on $J_{1}, \hat{f}$ restricts to a homomorphism $J_{1} \rightarrow J_{2}$. Thus $\hat{f}$ gives a morphism of extensions.

The uniqueness claim in (1) follows from (2) by taking $f=g$. And (3) follows from (1) and (2) So it remains to prove (2) Assume that we are in the situation of (2) Let $\mathrm{ev}_{0}, \mathrm{ev}_{1}: A_{2} \otimes V[t]^{\dagger} \rightrightarrows A_{2}$ and $\mathrm{ev}_{0}, \mathrm{ev}_{1}: E_{2} \otimes V[t]^{\dagger} \rightrightarrows E_{2}$ denote the evaluation homomorphisms. Form the pull-back pro-algebra

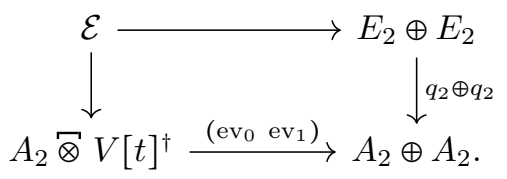


The universal property of the pull back gives pro-algebra homomorphisms

$$
\begin{gathered}
q:=\left(\mathrm{ev}_{0}, \mathrm{ev}_{1}, q_{2} \otimes \mathrm{id}_{V[t]^{\dagger}}\right)_{*}: E_{2} \otimes V[t]^{\dagger} \rightarrow \mathcal{E}, \\
\left(\hat{f}, \hat{g}, h \circ q_{1}\right)_{\star}: E_{1} \rightarrow \mathcal{E},
\end{gathered}
$$

because $\hat{f}$ and $\hat{g}$ lift $\mathrm{ev}_{t} \circ h$ for $t=0,1$, respectively. Let

$$
V[t]_{0}^{\dagger}:=\left\{\varphi \in V[t]^{\dagger}: \varphi(0)=0, \varphi(1)=0\right\} .
$$

We claim that $q$ is part of a semi-split extension of pro-algebras

$$
J_{2} \otimes V[t]_{0}^{\dagger} \rightarrow E_{2} \otimes V[t]^{\dagger} \rightarrow \mathcal{E} .
$$

To see this, we forget multiplications and treat everything as a projective system of bornological $V$-modules. In this category, a pro-linear section $s: A_{2} \rightarrow E_{2}$ for the semi-split extension $J_{2} \rightarrow E_{2} \rightarrow A_{2}$ gives a direct sum decomposition $E_{2} \cong J_{2} \oplus A_{2}$. And $V[t]^{\dagger} \cong V[t]_{0}^{\dagger} \oplus V \oplus V$, where the latter two summands are, say, spanned by the functions $1-t$ and $t$. This induces decompositions

$$
A_{2} \otimes V[t]^{\dagger} \cong\left(A_{2} \otimes V[t]_{0}^{\dagger}\right) \oplus A_{2} \oplus A_{2}, \quad E_{2} \otimes V[t]^{\dagger} \cong\left(E_{2} \otimes V[t]_{0}^{\dagger}\right) \oplus E_{2} \oplus E_{2},
$$

such that $\left(\mathrm{ev}_{0}, \mathrm{ev}_{1}\right)$ is the projection to the second and third summand both for $A_{2}$ and $E_{2}$. These direct sum decompositions imply

$$
E_{2} \otimes V[t]^{\dagger} \cong\left(J_{2} \otimes V[t]_{0}^{\dagger}\right) \oplus\left(A_{2} \otimes V[t]_{0}^{\dagger}\right) \oplus E_{2} \oplus E_{2} \cong\left(J_{2} \otimes V[t]_{0}^{\dagger}\right) \oplus \mathcal{E} .
$$

And this proves the claim.

Corollary 2.1.21 and Proposition 4.2.6 imply that the tensor product $J_{2} \otimes V[t]_{0}^{\dagger}$ is analytically nilpotent. Since $E_{1}$ is analytically quasi-free, the homomorphism $\left(\hat{f}, \hat{g}, h \circ q_{1}\right)$ lifts to a homomorphism $\hat{h}: E_{1} \rightarrow E_{2} \otimes V[t]^{\dagger}$ in the extension (4.3.11). This finishes the proof of (2)

Corollary 4.3.12. Any two analytically quasi-free, analytically nilpotent extensions of a pro-algebra are dagger homotopy equivalent.

Proof. By Proposition 4.3.10, there are morphisms of extensions in both directions which lift the identity map on $A$ and whose composite maps are dagger homotopic to the identity maps.

Proposition 4.3.13. Let $A \nrightarrow E \rightarrow B$ be an extension of pro-algebras. If $A$ and $B$ are isomorphic to projective systems of dagger algebras, then so is $E$. If $A$ and $B$ are analytically nilpotent, then so is $E$.

Proof. Being nilpotent mod $\pi$ is hereditary for pro-algebra extensions by Proposition 4.2.6. Hence the second statement follows from the first one. Its proof has several steps. First, we rewrite the given extension of pro-algebras as a projective limit of a projective system of algebra extensions. Similar ideas in a less specialised setting also appear in [4, Appendix].

Write $E$ and $B$ as projective systems of (torsion-free, complete bornological) algebras $\left(E_{n}, \gamma_{n, m}\right)$ and $\left(B_{n}, \beta_{n, m}\right)$ that are indexed by directed sets $N_{E}$ and $N_{B}$, respectively. By assumption, $B$ is isomorphic to a projective system of dagger algebras. We assume that we have picked this representative above, that is, each $B_{n}$ is a dagger algebra. We describe the pro-algebra morphism $E \rightarrow B$ by a coherent family of bounded homomorphisms $\varphi_{n}: E_{m(n)} \rightarrow B_{n}$ for all $n \in N_{B}$. Let $N:=$ $\left\{(m, n) \in N_{E} \times N_{B}: m \geq m(n)\right\}$. Define a partial order on $N$ by $\left(m_{1}, n_{1}\right) \geq\left(m_{2}, n_{2}\right)$ if $m_{1} \geq m_{2}, n_{1} \geq n_{2}, m_{1} \geq m\left(n_{2}\right)$, and $\beta_{n_{2}, n_{1}} \circ \varphi_{n_{1}} \circ \gamma_{m\left(n_{1}\right), m_{1}}=\varphi_{n_{2}} \circ \gamma_{m\left(n_{2}\right), m_{1}}$. 
This partially ordered set is directed because $N_{B}$ and $N_{E}$ are directed and the maps $\varphi_{n}$ for $n \in N$ form a morphism of projective systems. The objects $E_{m}$ and $B_{n}$ for $(m, n) \in N$ and the maps $\gamma_{m_{1}, m_{2}}$ and $\beta_{n_{1}, n_{2}}$ for $m_{1} \geq m_{2}$ and $n_{1} \geq n_{2}$ form projective systems $E^{\prime}$ and $B^{\prime}$ of bornological algebras. They are isomorphic to $E$ and $B$, respectively. The homomorphisms

$$
\varphi_{(m, n)}^{\prime}:=\varphi_{n} \circ \gamma_{m(n), m}: E_{(m, n)}^{\prime}=E_{m} \rightarrow B_{n}=B_{(m, n)}^{\prime}
$$

for $(m, n) \in N$ are coherent in the strong sense that

$$
\beta_{\left(m_{1}, n_{1}\right),\left(m_{2}, n_{2}\right)}^{\prime} \circ \varphi_{\left(m_{2}, n_{2}\right)}^{\prime}=\varphi_{\left(m_{1}, n_{1}\right)}^{\prime} \circ \gamma_{\left(m_{1}, n_{1}\right),\left(m_{2}, n_{2}\right)}^{\prime}
$$

for all $\left(m_{1}, n_{1}\right),\left(m_{2}, n_{2}\right) \in N$ with $\left(m_{1}, n_{1}\right) \leq\left(m_{2}, n_{2}\right)$. Here $\gamma^{\prime}$ and $\beta^{\prime}$ denote the structure maps of the projective systems $E^{\prime}$ and $B^{\prime}$, respectively. By construction, each $B_{n}^{\prime}$ is a dagger algebra.

By assumption, the inclusion $A \rightarrow E$ is the kernel of the morphism $E \rightarrow B$. This is isomorphic to the kernel of $\varphi^{\prime}: E^{\prime} \rightarrow B^{\prime}$. So $A$ is isomorphic to the projective system $A^{\prime}$ formed by the closed ideals $A_{n}^{\prime}:=\operatorname{ker} \varphi_{n} \subseteq E_{n}^{\prime}$ for $n \in N$ with the structure maps $\alpha_{n_{1}, n_{2}}^{\prime}=\left.\gamma_{n_{1}, n_{2}}^{\prime}\right|_{A_{n_{2}}}$ for $n_{1}, n_{2} \in N$ with $n_{1} \leq n_{2}$; and the canonical morphism $A^{\prime} \rightarrow E^{\prime}$ is the strongly coherent family of inclusion maps $A_{n}^{\prime} \rightarrow E_{n}^{\prime}$ for $n \in N$. Each $A_{n}^{\prime}$ is complete and torsion-free because $E_{n}^{\prime}$ and $B_{n}^{\prime}$ are (see $\mathbf{2 7}$. Theorem 2.3 and Lemma 4.2]).

The quotients $E_{n}^{\prime} / A_{n}^{\prime}$ with the structure maps $\dot{\gamma}_{n, m}^{\prime}$ induced by $\gamma_{n, m}^{\prime}$ form a projective system of complete bornological algebras, which is the cokernel for the inclusion $A^{\prime} \leftrightarrow E^{\prime}$. The map $\varphi_{n}^{\prime}$ for $n \in N$ descends to an injective, bounded homomorphism $\varrho_{n}: E_{n}^{\prime} / A_{n}^{\prime} \rightarrow B_{n}^{\prime}$. The pro-algebra morphism $\varrho=\left(\varrho_{n}\right)_{n \in N}$ is an isomorphism because $E \rightarrow B$ is assumed to be another cokernel for the map $A \rightarrow E$. Next, we modify our projective systems so that these become equalities; this replaces the quotients $E_{n}^{\prime} / A_{n}^{\prime}$ by dagger algebras. The inverse of $\varrho$ is given by a choice of $m(n) \in N$ for $n \in N$ and bounded homomorphisms $\psi_{n}: B_{m(n)}^{\prime} \rightarrow E_{n}^{\prime} / A_{n}^{\prime}$. Increasing $m(n)$ if necesessary, we may arrange that $\varrho_{n} \circ \psi_{n}=\beta_{n, m(n)}^{\prime}: B_{m(n)}^{\prime} \rightarrow B_{n}^{\prime}$ and $\psi_{n} \circ \varrho_{m(n)}=\dot{\gamma}_{n, m(n)}^{\prime}: E_{m(n)}^{\prime} / A_{m(n)}^{\prime} \rightarrow E_{n}^{\prime} / A_{n}^{\prime}$. Let $N^{\prime}:=\{(m, n) \in N \times N: m \geq m(n)\}$. For $(m, n) \in N^{\prime}$, pull the extension $A_{n}^{\prime} \nrightarrow E_{n}^{\prime} \rightarrow E_{n}^{\prime} / A_{n}^{\prime}$ back along $\psi_{n}$ as in Lemma 2.3.2 This gives a diagram of extensions of bornological $V$-modules

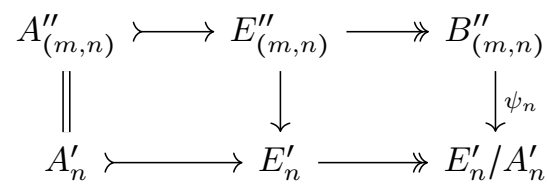

with $A_{(m, n)}^{\prime \prime}=A_{n}^{\prime}$ and $B_{(m, n)}^{\prime \prime}=B_{m}^{\prime}$. The latter is a dagger algebra because it is equal to $B_{m}$ for suitable $m \in N_{B}$ depending on $n \in N^{\prime}$. There is a unique bornological algebra structure on $E_{(m, n)}^{\prime \prime}$ for which all maps in this diagram are homomorphisms. We claim that $E_{(m, n)}^{\prime \prime}$ is complete. First, $A_{n}^{\prime}$ is closed in $E_{n}^{\prime}$ because $B_{n}^{\prime}$ is separated. Then $E_{n}^{\prime} / A_{n}^{\prime}$ is separated (see [27, Lemma 2.1]). Then $E_{(m, n)}^{\prime \prime}$ is closed in $B_{m}^{\prime} \oplus E_{n}^{\prime}$. And then $E_{(m, n)}^{\prime \prime}$ is complete by 27 , Theorem 2.3]. As above, there is a partial order on $N^{\prime}$ that makes it a directed set and such that $A_{n}^{\prime \prime} \rightarrow E_{n}^{\prime \prime} \rightarrow B_{n}^{\prime \prime}$ becomes a projective system of algebra extensions. This projective system is isomorphic to $A^{\prime} \nrightarrow E^{\prime} \rightarrow E^{\prime} / A^{\prime}$ because it is the pullback along the pro-algebra isomorphism $B^{\prime} \stackrel{\sim}{\rightarrow} E^{\prime} / A^{\prime}$. Thus it is isomorphic to the original extension $A \nrightarrow E \rightarrow B$. We 
have now replaced this pro-algebra extension by a projective system of algebras extensions where the quotients $B_{n}^{\prime \prime}$ are dagger algebras.

To simplify notation, we remove the primes now and assume that our proalgebra extension already comes to us as a projective system of algebra extensions $A_{n} \gg E_{n} \rightarrow B_{n}$, where $A_{n}$ and $E_{n}$ are torsion-free, complete bornological algebras and $B_{n}$ are dagger algebras for all $n \in N$. The dagger completions $E_{n}^{\dagger}$ for $n \in N$ form a projective system of dagger algebras, and the canonical maps $E_{n} \rightarrow E_{n}^{\dagger}$ form a pro-algebra morphism. We claim that this pro-algebra morphism is an isomorphism. Equivalently, for each $n \in N$ there are $m \in N$ with $m \geq n$ and a bounded homomorphism $\tilde{\gamma}_{n, m}: E_{m}^{\dagger} \rightarrow E_{n}$ such that the composite map $E_{m} \rightarrow E_{m}^{\dagger} \rightarrow E_{n}$ is $\gamma_{n, m}$; then the other composite map $E_{m}^{\dagger} \rightarrow E_{n} \rightarrow E_{n}^{\dagger}$ is the map on the dagger completions induced by $\gamma_{n, m}$, and these two equalities of compositions say that we are dealing with morphisms of pro-algebras inverse to each other.

Fix $n \in N$. We are going to build the following commuting diagram, where the dashed arrow is the desired map $\tilde{\gamma}_{n, m}$ :

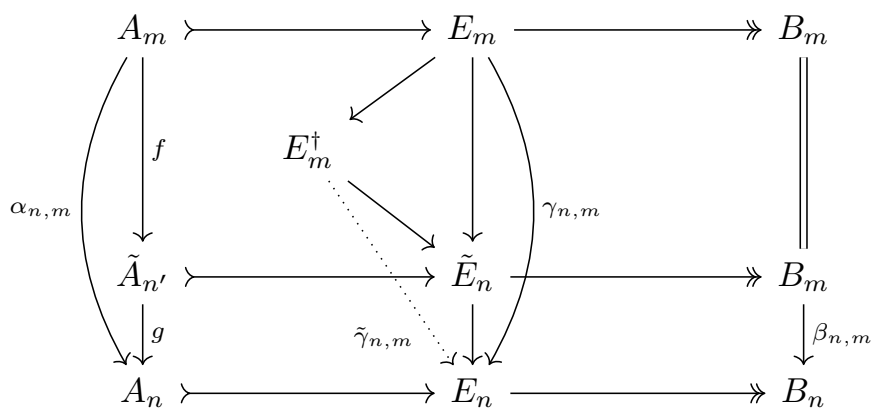

By assumption, $A$ is isomorphic to a projective system of dagger algebras $\left(\tilde{A}_{n^{\prime}}\right)_{n^{\prime} \in N^{\prime}}$. Therefore, there are $m \in N, n^{\prime} \in N^{\prime}$, and maps $f: A_{m} \rightarrow \tilde{A}_{n^{\prime}}$ and $g: \tilde{A}_{n^{\prime}} \rightarrow A_{n}$ such that $m \geq n$ and $g \circ f=\alpha_{n, m}: A_{m} \rightarrow A_{n}$. Let $\tilde{E}_{n}$ be the pushout bornological $V$-module of the maps $A_{m} \rightarrow E_{m}$ and $A_{m} \rightarrow \tilde{A}_{n^{\prime}}$. This fits in an extension of bornological $V$-modules $\tilde{A}_{n^{\prime}} \rightarrow \tilde{E}_{n} \rightarrow B_{m}$ by Lemma 2.3.2. Since $\tilde{A}_{n^{\prime}}$ and $B_{m}$ are torsion-free and complete, $\tilde{E}_{n}$ is complete by $\left[\mathbf{2 7}\right.$, Theorem 2.3]. Since $\tilde{A}_{n^{\prime}}$ is semi-dagger, the canonical map $E_{m} \rightarrow \tilde{E}_{n}$ remains bounded when we give $E_{m}$ the linear growth bornology relative to the ideal $A_{m}^{\prime}$. This bornology is equal to the absolute linear growth bornology on $E_{m}$ by Lemma 2.2 .6 because $B_{m}=E_{m} / A_{m}$ is a dagger algebra. Since $\tilde{E}_{n}$ is complete, the map $E_{m} \rightarrow E_{n}$ extends to a bounded $V$-module homomorphism $E_{m}^{\dagger} \rightarrow \tilde{E}_{n}$. By construction, the map $\gamma_{n, m}: E_{m} \rightarrow E_{n}$ agrees on $A_{m}$ with the composite map

$$
A_{m} \stackrel{f}{\rightarrow} \tilde{A}_{n^{\prime}} \stackrel{g}{\rightarrow} A_{n} \rightarrow E_{n} .
$$

Then the universal property of pushouts gives an induced bounded $V$-module homomorphism $\tau: \tilde{E}_{n} \rightarrow E_{n}$. Let $\tilde{\gamma}_{n, m}: E_{m}^{\dagger} \rightarrow E_{n}$ be the composite of the bounded $V$-module homomorphisms $E_{m}^{\dagger} \rightarrow \tilde{E}_{n}$ and $\tilde{E}_{n} \rightarrow E_{n}$ defined above. The composite map $E_{m} \rightarrow E_{m}^{\dagger} \rightarrow E_{n}$ is $\gamma_{n, m}$ by construction. This finishes the proof that $E_{n}$ is isomorphic to a projective system of dagger algebras.

4.4. The analytic tensor algebra. Let $R$ be a constant pro-algebra. The definitions of $\mathbb{H A}(R)$ and $\mathrm{HA}_{*}(R)$ use a certain pro-algebra $\mathcal{T} R$ defined by completing the tensor algebra $\mathrm{T} R$. We call $\mathcal{T} R$ the analytic tensor algebra of $R$. We 
show that there is a semi-split analytically nilpotent extension $\mathcal{J} R \nrightarrow \mathcal{T} R \rightarrow R$ and that $\mathcal{T} R$ is analytically quasi-free. Since it is not more difficult, we extend the construction of the analytic tensor algebra to pro-algebras right away.

Definition 4.4.1. Let $R=\left(R_{n}, \alpha_{m, n}\right)_{m, n \in N}$ be a pro-algebra. Extending the tensor algebra construction to pro-algebras gives a natural semi-split pro-algebra extension $\mathrm{J} R \rightarrow \mathrm{T} R \rightarrow R$ with $\mathrm{T} R=\left(\mathrm{T} R_{n}\right)_{n \in N}$ and $\mathrm{J} R=\left(\mathrm{J} R_{n}\right)_{n \in N}$. For each $n \in N$, we form the tube algebras $\mathcal{U}\left(\mathrm{T} R,(\mathrm{~J} R)^{l}\right)$ with the ideals $\mathcal{U}\left(\mathrm{J} R,(\mathrm{~J} R)^{l}\right)$, and their relative dagger completions $\left(\mathcal{U}\left(\mathrm{T} R,(\mathrm{~J} R)^{l}\right), \mathcal{U}\left(\mathrm{J} R,(\mathrm{~J} R)^{l}\right)\right)^{\dagger}$. These form a pro-algebra indexed by the product set $N \times \mathbb{N}$, which we call the analytic tensor algebra of $R$ and denote by $\mathcal{T} R$.

LEMMA 4.4.2. The canonical homomorphism $p: \mathrm{T} R \rightarrow R$ extends uniquely to a pro-algebra homomorphism $\tilde{p}: \mathcal{T} R \rightarrow R$. The composite $\sigma_{\text {an }}$ of the pro-linear map $\sigma_{R}: R \rightarrow \mathrm{T} R$ and the canonical homomorphism $\mathrm{T} R \rightarrow \mathcal{T} R$ is a section for $\tilde{p}$.

Proof. Fix $n \in N$ and $l \in \mathbb{N}^{*}$. The canonical homomorphism $\mathrm{T} R_{n} \rightarrow R_{n}$ vanishes on $J R_{n}$. Then it extends uniquely to the tube algebra $\mathcal{U}\left(\mathrm{T} R_{n},\left(\mathrm{~J} R_{n}\right)^{l}\right)$ by Proposition 4.2.4. This extension vanishes on $\mathcal{U}\left(\mathrm{J} R_{n},\left(\mathrm{~J} R_{n}\right)^{l}\right)$. Then it remains bounded for the linear growth bornology relative to this ideal and extends uniquely to a homomorphism on the relative dagger completion. These maps for all $n$ and $l$ form a morphism of pro-algebras $\tilde{p}: \mathcal{T} R \rightarrow R$. The canonical maps $\sigma_{R_{n}}: R_{n} \rightarrow \mathrm{T} R_{n}$ form a pro-linear section for $p: \mathrm{T} R \rightarrow R$. Composing with the canonical map $\mathrm{T} R \rightarrow \mathcal{T} R$ gives a section for $\tilde{p}$.

Definition 4.4.3. Let $\mathcal{J} R$ be the kernel of $\tilde{p}: \mathcal{T} R \rightarrow R$.

Lemma 4.4 .2 implies that there is a semi-split extension of pro-algebras

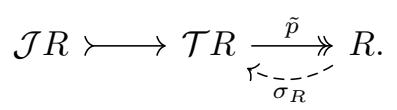

Proposition 4.4.4. The pro-algebra $\mathcal{J} R$ is analytically nilpotent.

Proof. Let $m \in \mathbb{N}^{*}$. The linear growth bornology on $\mathcal{U}\left(\mathrm{T} R,(\mathrm{~J} R)^{m}\right)$ relative to $\mathcal{U}\left(\mathrm{J} R,(\mathrm{~J} R)^{m}\right)$ restricts to the "absolute" linear growth bornology on $\mathcal{U}\left(\mathrm{J} R,(\mathrm{~J} R)^{m}\right)$ by Lemma 2.2.5 The tensor algebra is bornologically torsion-free by Remark 2.6 .2 Then so is $\mathcal{U}\left(\mathrm{T} R,(\mathrm{~J} R)^{m}\right)$ by the definition of the bornology on the tube algebra. Then the relative linear growth bornology on it is torsion-free by Lemma 2.2.7 and this property is preserved by completions (see [27. Theorem 4.6]). Therefore, the completion of $\mathcal{U}\left(\mathrm{J} R,(\mathrm{~J} R)^{m}\right)$ in the linear growth bornology is a dagger algebra. Then $\mathcal{J} R$ is a pro-dagger algebra. And $\mathcal{U}\left(\mathrm{J} R,(\mathrm{~J} R)^{\infty}\right)$ is nilpotent $\bmod \pi$ by Proposition 4.2.4. This remains unaffected when we equip the tube algebras with the linear growth bornology and complete.

REMARK 4.4.5. Let $R=\left(R_{n}, \alpha_{m, n}\right)_{m, n \in N}$ be a projective system of dagger algebras. Since $\mathcal{U}\left(\mathrm{T} R,(\mathrm{~J} R)^{l}\right) / \mathcal{U}\left(\mathrm{J} R,(\mathrm{~J} R)^{l}\right) \cong R$ is semi-dagger, the linear growth bornology on $\mathcal{U}\left(\mathrm{T} R,(\mathrm{~J} R)^{l}\right)$ is equal to the linear growth bornology relative to $\mathcal{U}\left(\mathrm{J} R,(\mathrm{~J} R)^{l}\right)$ by Lemma 2.2.6. Hence $\mathcal{T} R$ is also equal to the "absolute" dagger completion,

$$
\mathcal{T} R \cong \mathcal{U}\left(\mathrm{T} R,(\mathrm{~J} R)^{\infty}\right)^{\dagger} .
$$


Proposition 4.4.6. The analytic tensor algebra $\mathcal{T} R$ is analytically quasi-free and quasi-free. The bimodule $\bar{\Omega}^{1}(\mathcal{T} R)$ is isomorphic to the free bimodule on $R$, that is,

$$
(\mathcal{T} R)^{+} \otimes R \otimes(\mathcal{T} R)^{+} \cong \bar{\Omega}^{1}(\mathcal{T} R)
$$

the isomorphism is the map $\omega \otimes x \otimes \eta \mapsto \omega \cdot\left(\mathrm{d} \sigma_{R}(x)\right) \cdot \eta$. And the following maps are isomorphisms of left or right $\mathcal{T} R$-modules, respectively:

$$
\begin{array}{ll}
(\mathcal{T} R)^{+} \otimes R \stackrel{\sim}{\rightarrow} \mathcal{T} R, & \omega \otimes x \mapsto \omega \odot \sigma_{R}(x), \\
R \bar{\otimes}(\mathcal{T} R)^{+} \stackrel{\simeq}{\rightarrow} \mathcal{T} R, & x \otimes \omega \mapsto \sigma_{R}(x) \odot \omega .
\end{array}
$$

Proof. Let $J \nrightarrow E \stackrel{q}{\rightarrow} \mathcal{T} R$ be a semi-split, analytically nilpotent pro-algebra extension. Pull it back along the inclusion $\mathcal{J} R \leftrightarrow \mathcal{T} R$ to a pro-algebra extension $J \nrightarrow K \rightarrow \mathcal{J} R$ and identify $K$ with an ideal in $E$. Since $J$ and $\mathcal{J} R$ are analytically nilpotent, so is $K$ by Proposition 4.3.13. Let $s: \mathcal{T} R \rightarrow E$ be a pro-linear section and let $\sigma_{R}: R \rightarrow \mathcal{T} R$ be the canonical pro-linear section. The pro-linear map $s \circ \sigma_{R}$

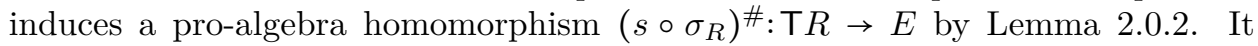
satisfies $q \circ\left(s \circ \sigma_{R}\right)^{\#}=\sigma_{R}^{\#}$, and $\sigma_{R}^{\#}: \mathrm{T} R \rightarrow \mathcal{T} R$ is the canonical homomorphism because $\sigma_{R}^{\#}$ and the inclusion map agree on the image of $R$ in T $R$. In particular, $\left(s \circ \sigma_{R}\right)^{\#}$ maps $J R$ into $K \triangleleft E$. Since $K$ is nilpotent $\bmod \pi$, Proposition 4.2 .4 shows that $\left(s \circ \sigma_{R}\right)^{\#}$ extends to the tube algebra $\mathcal{U}\left(\mathrm{T} R,(\mathrm{~J} R)^{\infty}\right)$, in such a way that $\mathcal{U}\left(\mathrm{J} R,(\mathrm{~J} R)^{\infty}\right)$ is mapped to $K$. And since $K$ is a pro-dagger algebra, the criterion in Proposition 2.2.9 shows that the morphism $\mathcal{U}\left(\mathrm{T} R,(\mathrm{~J} R)^{\infty}\right) \rightarrow E$ extends uniquely to the dagger completion relative to $\mathcal{U}\left(\mathrm{J} R,(\mathrm{~J} R)^{\infty}\right)$. This gives a pro-algebra morphism $\mathcal{T} R \rightarrow E$ that is a section for the extension $J \nrightarrow E \stackrel{q}{\rightarrow} \mathcal{T} R$. So $\mathcal{T} R$ is analytically quasi-free.

If $h: R \rightarrow E$ is any pro-linear map with $q \circ h=\sigma_{R}$, then the argument above shows that $h^{\#}: \mathrm{T} R \rightarrow E$ extends uniquely to a pro-algebra morphism $\mathcal{T} R \rightarrow E$ that is a section for the extension. Conversely, any multiplicative section $g: \mathcal{T} R \rightarrow E$ is of this form for $h:=g \circ \sigma_{R}$. Thus the multiplicative sections for the extension $J \nrightarrow E \stackrel{q}{\rightarrow} \mathcal{T} R$ are in bijection with pro-linear maps $R \rightarrow E$ with $q \circ h=\sigma_{R}$. Any such pro-linear map is equal to $s \circ \sigma_{R}+h_{0}$ for a unique pro-linear map $h_{0}: R \rightarrow J$. So multiplicative sections for our extension are in bijection with pro-linear maps $R \rightarrow J$. Combined with Lemma 2.5.1, we get a natural bijection for all $\mathcal{T} R$-bimodules $M$ between pro-bimodule homomorphisms $\bar{\Omega}^{1}(\mathcal{T} R) \rightarrow M$ and pro-linear maps $R \rightarrow M$. Thus $\widetilde{\Omega}^{1}(\mathcal{T} R)$ is isomorphic to the free bimodule on $R$, which is $(\mathcal{T} R)^{+} \otimes R \nabla(\mathcal{T} R)^{+}$. And this isomorphism is indeed induced by the map $\omega \otimes x \otimes \eta \mapsto \omega \cdot\left(\mathrm{d} \sigma_{R}(x)\right) \cdot \eta$.

Now let $M$ be a left $\mathcal{T} R$-module. Turn $M$ into a $\mathcal{T} R$-bimodule by taking the zero map as right module structure. Then a bimodule derivation $\mathcal{T} R \rightarrow M$ is just a left module map. Therefore, left module homomorphisms $\mathcal{T} R \rightarrow M$ are in bijection with pro-linear maps $R \rightarrow M$. Thus the map

$$
(\mathcal{T} R)^{+} \otimes R, \quad \omega \otimes x \mapsto \omega \odot \sigma_{R}(x),
$$

is an isomorphism of left $\mathcal{T} R$-modules. Here we have written $\odot$ for the multiplication in $\mathcal{T} R$ because we will later use these formulas when $\mathcal{T} R$ is identified with $\Omega^{\mathrm{ev}} R$ with the Fedosov product. A similar argument works for right modules.

We now describe the analytic tensor algebra and its bornology more concretely. For this, we assume that $R$ is a torsion-free, complete bornological algebra. A 
projective system $\left(R_{n}\right)_{n \in N}$ is treated by applying the following discussion to $R_{n}$ for each $n \in N$. We identify T $R$ with $\Omega^{\mathrm{ev}} R$ with the Fedosov product as in Section 2.6 Recall that the isomorphism $\mathrm{T} R \cong \Omega^{\mathrm{ev}} R$ maps the ideal $\mathrm{J} R^{m}$ onto $\oplus_{n \geq m} \Omega^{2 n} R$. Thus $\mathcal{U}\left(\mathrm{T} R,(\mathrm{~J} R)^{m}\right)$ is spanned by $\pi^{-j} \Omega^{2 n} R$ with $n \geq m \cdot j$. And $\mathcal{U}\left(\mathrm{J} R,(\mathrm{~J} R)^{m}\right)$ is spanned by $\pi^{-j} \Omega^{2 n} R$ with $n \geq m \cdot j$ and $n \geq 1$. Equivalently,

$$
\mathcal{U}\left(\mathrm{T} R,(\mathrm{~J} R)^{m}\right)=\sum_{n=0}^{\infty} \pi^{-\lfloor n / m\rfloor} \Omega^{2 n} R, \quad \mathcal{U}\left(\mathrm{J} R,(\mathrm{~J} R)^{m}\right)=\sum_{n=1}^{\infty} \pi^{-\lfloor n / m\rfloor} \Omega^{2 n} R
$$

The following lemma estimates the growth of Fedosov products in $\Omega R$ :

Lemma 4.4.9. Let $R$ be an algebra and let $M \subseteq R$ be a submodule. Let $i_{0}, \ldots, i_{n} \geq$ 1 and $i:=i_{0}+\cdots+i_{n}$. Then

$$
\Omega^{i_{0}} M \odot \cdots \odot \Omega^{i_{n}} M \subseteq \bigoplus_{j=0}^{n} \Omega^{i+2 j}\left(M^{(3)}\right) .
$$

Proof. As in the proof of [26. Theorem 5.11], we show the more precise estimate

$$
\Omega^{i_{0}} M \odot \cdots \odot \Omega^{i_{n}} M \subseteq \bigoplus_{j=0}^{n}\left(M^{(2)}\right)^{+} \mathrm{d}\left(M^{(3)}\right)^{i+2 j}
$$

by induction on $n$. This is trivial for $n=0$. The induction step uses 2.6.5 and

$$
\Omega^{i} M \odot\left(M^{(2)}\right)^{+} \subseteq\left(M^{(2)}\right)^{+} \mathrm{d}\left(M^{(3)}\right)^{i}+(\mathrm{d} M)^{i+1} \mathrm{~d}\left(M^{(2)}\right) .
$$

Proposition 4.4.11. Let $R$ be a torsion-free bornological algebra and $m \geq 1$. If $M \subseteq R$ is bounded, $\alpha \in \mathbb{Q} \cap(0,1 / m)$, and $f \in \mathbb{N}_{0}$, then define

$$
D_{m}(M, \alpha, f):=\bigoplus_{n=0}^{\infty} \pi^{-\lfloor\min \{n / m, \alpha \cdot n+f\}\rfloor} \Omega^{2 n} M .
$$

These are $V$-submodules of $\mathcal{U}\left(\mathrm{T} R,(\mathrm{~J} R)^{m}\right)$ that cofinally generate its linear growth bornology relative to the ideal $\mathcal{U}\left(\mathrm{J} R,(\mathrm{~J} R)^{m}\right)$.

Proof. Let $M \subseteq R$ be bounded, $\alpha \in \mathbb{Q} \cap(0,1 / m)$, and $f \in \mathbb{N}_{0}$. Equation 4.4.8) implies $D_{m}(M, \alpha, f) \subseteq \mathcal{U}\left(\mathrm{T} R, \mathrm{~J} R^{m}\right)$. Our first goal is to show that $D_{m}(M, \alpha, f)$ has linear growth relative to $\mathcal{U}\left(\mathrm{J} R, \mathrm{~J} R^{m}\right)$. Let $e \geq 1$. We claim that

$$
M^{+} \cdot\left(\sum_{n=1}^{e m} \pi^{-\lfloor n / m\rfloor}(\mathrm{d} M \mathrm{~d} M)^{n}\right)^{\diamond}=\bigoplus_{n=1}^{\infty} \pi^{-\lfloor n / m\rfloor+\left[\frac{n}{e m}\right]-1} \Omega^{2 n} M .
$$

By definition, the left hand side is spanned by Fedosov products

$$
\begin{aligned}
& \pi^{j-1-\left\lfloor i_{1} / m\right\rfloor-\cdots-\left\lfloor i_{j} / m\right\rfloor} M^{+} \odot(\mathrm{d} M, \mathrm{~d} M)^{i_{1}} \odot \cdots \odot(\mathrm{d} M, \mathrm{~d} M)^{i_{j}} \\
&=\pi^{j-1-\left\lfloor i_{0} / m\right\rfloor-\cdots-\left\lfloor i_{j} / m\right\rfloor} \Omega^{2\left(i_{1}+\cdots+i_{j}\right)}(M)
\end{aligned}
$$

for $j \geq 1$ and $1 \leq i_{1}, \ldots, i_{j} \leq e m$. These contribute to $\Omega^{2 n} M$ if $i_{1}+\cdots+i_{j}=n$. For fixed $n$ and $j$, the sum of floors $\left\lfloor i_{1} / m\right\rfloor+\cdots+\left\lfloor i_{j} / m\right\rfloor$ is maximal if all but one of the $i_{j}$ are divisible by $m$, and then it becomes $\lfloor n / m\rfloor$. For fixed $n$, the term $j-1-\lfloor n / m\rfloor$ becomes minimal if $j$ is minimal. Equivalently, we choose $i_{j}=e m$ for all but one $j$, and then $j=\lceil n / e m\rceil$. This finishes the proof of (4.4.13).

The right hand side in 4.4.13) is one of the generators of the linear growth bornology relative to $\mathcal{U}\left(\mathrm{J} R,(\mathrm{~J} R)^{m}\right)$. For fixed $\alpha<1 / m$ and $f$ as above, there is 
$e \in \mathbb{N}^{*}$ with $1 / m-1 /(e m)>\alpha$. Then there is $k \in \mathbb{N}$ with

$$
\lfloor n / m\rfloor-\left\lceil\frac{n}{e m}\right\rceil+1 \geq\lfloor\min \{n / m, \alpha \cdot n+f\}\rfloor
$$

for $n>k$. Then

$$
D_{m}(M, \alpha, f) \subseteq \sum_{n=0}^{k} \pi^{-\lfloor\min \{n / m, \alpha \cdot n+f\}\rfloor} \Omega^{2 n} M+M^{+} \cdot\left(\sum_{n=1}^{e m} \pi^{-\lfloor n / m\rfloor}(\mathrm{d} M \mathrm{~d} M)^{n}\right)^{\diamond} .
$$

The first, finite sum is already bounded in $\mathcal{U}\left(\mathrm{T} R, \mathrm{~J} R^{m}\right)$. Therefore, $D_{m}(M, \alpha, f)$ has linear growth relative to $\mathcal{U}\left(\mathrm{J} R,(\mathrm{~J} R)^{m}\right)$.

Now let $S$ be any $V$-submodule of $\mathcal{U}\left(\mathrm{T} R, \mathrm{~J} R^{m}\right)$ that has linear growth relative to $\mathcal{U}\left(\mathrm{J} R,(\mathrm{~J} R)^{m}\right)$. We claim that $S$ is contained in $D_{m}(M, \alpha, f)$ for suitable $M, \alpha, f$. By definition of the relative linear growth bornology, there are $k, e \in \mathbb{N}$ and a bounded submodule $M \subseteq R$ such that $S$ is contained in the sum of $\sum_{n=0}^{k} \pi^{-\lfloor n / m\rfloor} \Omega^{2 n} M$ and $\left(\sum_{i=1}^{e m} \pi^{-\left\lfloor\frac{i}{m}\right\rfloor} \Omega^{2 i} M\right)^{\diamond}$. The latter is spanned by Fedosov products

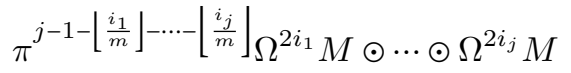

with $j \in \mathbb{N}^{*}, 1 \leq i_{1}, \ldots, i_{j} \leq e m$. By Lemma $4.4 .9 \Omega^{2 i_{1}} M \odot \cdots \Omega^{2 i_{j}} M$ is contained in the sum of $\Omega^{2 n}\left(M^{(3)}\right)$, where $n$ lies between $i:=\sum_{k=1}^{j} i_{k}$ and $i+j$. As above, the sum of the floors $\left\lfloor i_{k} / m\right\rfloor$ for fixed $i$ is maximal if all but one $i_{k}$ are divisible by $m$, and then it is $\lfloor i / m\rfloor$. The constraints $i_{k} \leq e m$ are equivalent to the constraint $i \leq j \cdot \mathrm{em}$. So $S$ is contained in the sum of $\pi^{j-1-\lfloor i / m\rfloor} \Omega^{2 n}\left(M^{(3)}\right)$ with $i \leq n \leq i+j$ and $i \leq j \cdot \mathrm{em}$. For fixed $n, j$, the exponent $j-1-\lfloor i / \mathrm{m}\rfloor$ is minimal if $i$ is maximal, so we may assume that $i$ is the minimum of $n$ and $j e m$. Then the optimal choice for $j$ is the minimal one, which is $\lceil n /(e m)\rceil$ if $i=n$ and $j=\lceil n /(e m+1)\rceil$ if $i=j e m$. The resulting exponents of $\pi$ become $\lceil n /(\mathrm{em})\rceil-1-\lfloor n / m\rfloor$ in the first case and $\lceil n /(e m+1)\rceil-1-\lceil n /(e m+1)\rceil \cdot e$ in the second. If $\alpha>1 / m-1 /(e m)$ and $n$ is large enough, then both terms are greater or equal $-\lfloor\alpha n\rfloor$. Choosing $f$ big enough, we may arrange that both are greater or equal $-\lfloor\min \{n / m, \alpha n+f\}\rfloor$ for all $n \in \mathbb{N}$. Then $S \subseteq D_{m}\left(M^{(3)}, \alpha, f\right)$.

Corollary 4.4.14. For $m \in \mathbb{N}^{*}$, let $\mathcal{B}_{m}$ be the bornology on $\mathcal{U}\left(\mathrm{T} R, \mathrm{~J} R^{m}\right)$ that contains a subset if and only if it is contained in $\left.\oplus_{n=0}^{\infty} \pi^{-\left\lfloor\frac{n}{m}\right.}\right\rfloor_{\Omega^{2 n}} M$ for some bounded $V$-submodule $M \subseteq R$. This bornology makes $\mathcal{U}\left(\mathrm{T} R, \mathrm{~J} R^{m}\right)$ a torsion-free bornological algebra. The projective system of bornological algebras $\left(\mathcal{U}\left(\mathrm{T} R, \mathrm{~J} R^{m}\right), \mathcal{B}_{m}\right)_{m \in \mathbb{N}^{*}}$ is isomorphic to the projective system formed by $\mathcal{U}\left(\mathrm{T} R, \mathrm{~J} R^{m}\right)$ with the linear growth bornology relative to $\mathcal{U}\left(\mathrm{J} R, \mathrm{~J} R^{m}\right)$.

Proof. By Lemma 4.4.9 the Fedosov product is bounded for the bornology $\mathcal{B}_{m}$. The subsets $D_{m}(M, \alpha, f)$ in 4.4 .12 are clearly in $\mathcal{B}_{m}$. Conversely,

$$
\bigoplus_{n=0}^{\infty} \pi^{-\left\lfloor\frac{n}{m+1}\right\rfloor} \Omega^{2 n} M=D_{m}\left(M, \frac{1}{m+1}, 0\right) .
$$

Thus any subset in $\mathcal{B}_{m+1}$ is mapped to a subset of $\mathcal{U}\left(\mathrm{T} R, \mathrm{~J} R^{m}\right)$ with linear growth relative to $\mathcal{U}\left(\mathrm{J} R, \mathrm{~J} R^{m}\right)$. The asserted isomorphism of projective systems follows.

Now we can describe the completion $\mathcal{T} R$. Recall that $\bar{\Omega}^{n} R$ denotes the completion $R^{+} \otimes R^{\Phi^{\otimes}}$ of $\Omega^{n} R=R^{+} \otimes R^{\otimes n}$. For $m \in \mathbb{N}^{*}$ and a bounded $V$-submodule $M \subseteq R$, the canonical map $\bar{\Omega}^{2 n} M \rightarrow \bar{\Omega}^{2 n} R$ is injective by Proposition 2.4.5. Then we may view $\prod_{n=0}^{\infty} \pi^{-\left\lfloor\frac{n}{m}\right\rfloor} \widetilde{\Omega}^{2 n} M$ as a $V$-submodule of $\prod_{n=0}^{\infty} \widetilde{\Omega}^{2 n} R \otimes F$. Let $\widetilde{\Omega}^{\mathrm{ev}}(R)_{m}$ be the 
union of $\prod_{n=0}^{\infty} \pi^{-\left\lfloor\frac{n}{m}\right\rfloor} \widetilde{\Omega}^{2 n} M$ for all bounded $V$-submodules $M \subseteq R$, with the bornology where a subset is bounded if and only if it is contained in $\prod_{n=0}^{\infty} \pi^{-\left\lfloor\frac{n}{m}\right\rfloor} \Omega^{2 n} M$ for some bounded $V$-submodules $M \subseteq R$. These form a decreasing sequence of subalgebras with bounded inclusion maps $\bar{\Omega}^{\mathrm{ev}}(R)_{m+1} \hookrightarrow \bar{\Omega}^{\mathrm{ev}}(R)_{m}$.

Proposition 4.4.15. If $R$ is a torsion-free, complete bornological algebra, then $\mathcal{T} R$ is naturally isomorphic to the projective system of complete bornological algebras $\left(\bar{\Omega}^{\mathrm{ev}}(R)_{m}\right)_{m \in \mathbb{N}^{*}}$.

Proof. We shall use the explicit description of the relative linear growth bornology in Proposition 4.4.11 Each $\pi^{-\lfloor n / m\rfloor} \Omega^{2 n} R$ is a direct summand of $\mathcal{U}\left(\mathrm{T} R, \mathrm{~J} R^{m}\right)$, and the projection is bounded in the linear growth bornology relative to $\mathcal{U}\left(\mathrm{J} R, \mathrm{~J} R^{m}\right)$. This gives us maps from the completed tube to $\pi^{-\lfloor n / m\rfloor} \widetilde{\Omega}^{2 n} R$ for all $n \in \mathbb{N}$. It is easy to see that the $\pi$-adic completion of $D_{m}(M, \alpha, f)$ is isomorphic to the subspace of $\prod_{n=0}^{\infty} \pi^{-\lfloor n / m\rfloor} \widetilde{\Omega}^{2 n} M$ consisting of all $\left(\omega_{n}\right)_{n \in \mathbb{N}}$ for which there is a sequence $\left(h_{j}\right)_{j \in \mathbb{N}}$ in $\mathbb{N}$ with $\lim h_{j}=\infty$ and $\omega_{n} \in \pi^{-\lfloor\min \{n / m, \alpha \cdot n+f\}\rfloor+h_{n}} \bar{\Omega}^{2 n} M$ for all $n \in \mathbb{N}$. Any such subset is bounded in $\widetilde{\Omega}^{\mathrm{ev}}(R)_{m}$. Conversely, any bounded subset in $\widetilde{\Omega}^{\mathrm{ev}}(R)_{m+1}$ is contained in a subset of this form with $f=0$ and $m<1 / \alpha<m+1$. Therefore, the projective system formed by the relative dagger completions $\left(\mathcal{U}\left(\mathrm{T} R, \mathrm{~J} R^{m}\right), \mathcal{U}\left(\mathrm{J} R, \mathrm{~J} R^{m}\right)\right)^{\dagger}$ is isomorphic to the projective system $\left(\bar{\Omega}^{\mathrm{ev}}(R)_{m}\right)_{m \in \mathbb{N}^{*}}$.

4.5. Pro-Linear maps with nilpotent curvature. Let $R$ and $S$ be proalgebras. We are going to describe pro-algebra homomorphisms $\mathcal{T} R \rightarrow S$ through a certain class of pro-linear maps $R \rightarrow S$, namely, those with analytically nilpotent curvature. This follows rather easily from the concrete description of the relative linear growth bornology above. The main issue is to define analytically nilpotent curvature. We begin with the analogue of nilpotent curvature mod $\pi$.

DeFinition 4.5.1. Let $X=\left(X_{n^{\prime}}\right)_{n^{\prime} \in N^{\prime}}$ be a bornological pro-module and $S=$ $\left(S_{n}\right)_{n \in N}$ a pro-algebra and let $\omega: X \rightarrow S$ be a pro-linear map. We call $\omega$ nilpotent $\bmod \pi$ if, for every $n \in N$, there is $m \in \mathbb{N}^{*}$ such that the following composite is zero:

$$
X^{\otimes m} \stackrel{\omega^{\otimes m}}{\longrightarrow} S_{m}^{\otimes m} \stackrel{\text { mult }}{\longrightarrow} S_{m} \rightarrow S_{m} / \pi S_{m}
$$

here mult denotes the $m$-fold multiplication map of $S$.

Let $\omega: X \rightarrow S$ be nilpotent $\bmod \pi$ and represent $\omega$ by a coherent family of bounded $V$-module maps $\omega_{n}: X_{r(n)} \rightarrow S_{n}$ with $r(n) \in N^{\prime}$ for $n \in N$. For $n \in N$ and $n^{\prime} \in N^{\prime}$ with $n^{\prime} \geq r(n)$, let $\omega_{n, n^{\prime}}: X_{n^{\prime}} \rightarrow S_{n}$ be the composite map $X_{n^{\prime}} \rightarrow X_{r(n)} \rightarrow S_{n}$. Let $n \in N$ and choose $m$ so that the map in 4.5.2 vanishes. Then there is $n^{\prime} \in N^{\prime}$ with $n^{\prime} \geq r(n)$ such that the composite map $X_{n^{\prime}}^{\otimes m} \rightarrow S_{n}^{\otimes m} \rightarrow S_{n} \rightarrow S_{n} / \pi S_{n}$ vanishes. That is, $\omega_{n, n^{\prime}}\left(x_{1}\right) \cdots \omega_{n, n^{\prime}}\left(x_{m}\right) \in \pi \cdot S_{n}$ for all $x_{1}, \ldots, x_{m} \in X_{n^{\prime}}$. Let $M \subseteq X_{n^{\prime}}$ be bounded. Since $\omega_{n, n^{\prime}}$ is bounded and $S_{n}$ is torsion-free, it follows that $\omega_{n, n^{\prime}}(M)^{m} \subseteq \pi S_{n}$ and that $\pi^{-1} \cdot \omega_{n, n^{\prime}}(M)^{m} \subseteq S_{n}$ is bounded. Then

$$
\omega_{n, n^{\prime}}(M)_{e}:=\sum_{j=1}^{e m} \pi^{-\lfloor j / m\rfloor} \omega_{n, n^{\prime}}(M)^{j}
$$

is bounded for every $e \geq 1$.

DeFinition 4.5.4. Let $X=\left(X_{m}\right)_{m \in N^{\prime}}$ be a bornological pro-module and $S=$ $\left(S_{n}\right)_{n \in N}$ a pro-algebra and let $\omega: X \rightarrow S$ be a pro-linear map. Represent $\omega$ by a 
coherent family of bounded $V$-module maps $\omega_{n, n^{\prime}}: X_{n^{\prime}} \rightarrow S_{n}$ as above. The map $\omega$ is called analytically nilpotent if, for every $n$, there are $m \in \mathbb{N}^{*}$ and $n^{\prime} \in N^{\prime}$ with $n^{\prime} \geq r(n)$ such that for any bounded subset $M \subseteq X_{n^{\prime}}$, the subset

$$
\sum_{j=0}^{\infty} \pi^{-\lfloor j / m\rfloor} \omega_{n, n^{\prime}}(M)^{j} \subseteq S_{n} \otimes F
$$

is bounded in $S_{n}$.

Proposition 4.5.5. Let $R$ and $S$ be pro-algebras and let $f: R \rightarrow S$ be a pro-linear map. Let $\omega: R \otimes R \rightarrow S, x \otimes y \mapsto f(x \cdot y)-f(x) \cdot f(y)$, be its curvature. There is a pro-algebra homomorphism $f^{\#}: \mathcal{T} R \rightarrow S$ with $f=f^{\#} \sigma_{R}=f$ if and only if $\omega$ is analytically nilpotent.

Proof. Write $R=\left(R_{n^{\prime}}\right)_{n^{\prime} \in N^{\prime}}$ and $S=\left(S_{n}\right)_{n \in N}$ as projective systems of algebras. Then $\mathcal{T} R$ is the completion of the projective system of bornological algebras $T:=$ $\left(\mathcal{U}\left(\mathrm{T} R_{n^{\prime}}, \mathrm{J} R_{n^{\prime}}^{m}\right), \mathcal{B}_{m}\right)_{n^{\prime} \in N^{\prime}, m \in \mathbb{N}^{*}}$ with the bornologies $\mathcal{B}_{m}$ in Corollary 4.4.14 Since $S$ is complete, any homomorphism of projective systems of bornological algebras $T \rightarrow S$ extends uniquely to $\mathcal{T} R$. Since $S$ is torsion-free, such a homomorphism $T \rightarrow S$ is determined by its restriction to TR. Then there is a unique pro-linear map $f: R \rightarrow S$ such that the homomorphism is $f^{\#}: \mathrm{T} R \rightarrow S$ as in 2.6.7). Corollary 4.4.14 shows that $f^{\#}$ extends to a homomorphism $T \rightarrow S$ if and only if $f$ has analytically nilpotent curvature.

Corollary 4.5.6. Let $f: R \rightarrow S, g: S \rightarrow T$ be pro-linear maps and let $U$ be a projective system of dagger algebras. If $f$ and $g$ have analytically nilpotent curvature, then so do $g \circ f$ and $f \otimes U: R \Phi U \rightarrow S \Phi U$.

Proof. The assertion about $g \circ f$ follows as in the proof of [26. Theorem 5.23], using [27, Theorems 3.7 and 4.5]. Since $f$ has analytically nilpotent curvature, there is a homomorphism $f^{\#}: \mathcal{T} R \rightarrow S$ with $f^{\#} \circ \sigma_{R}=f$. The extension

$$
(\mathcal{J} R) \otimes U \nrightarrow(\mathcal{T} R) \otimes U \rightarrow R \Phi U
$$

is analytically nilpotent because $(\mathcal{J} R) \otimes U$ is nilpotent $\bmod \pi$ by Proposition 4.2 .6 and a pro-dagger algebra by the extension of Corollary 2.1.21 to projective systems. The pro-linear section $\sigma_{R} \bar{\otimes} U$ induces a homomorphism $\mathcal{T}(R \bar{\otimes} U) \rightarrow(\mathcal{T} R) \bar{\otimes} U$ which, when composed with $f^{\#}$, gives a homomorphism $\mathcal{T}(R \bar{\otimes} U) \rightarrow S$ that extends $f^{\bar{\nabla} \otimes} U$. Thus $f \otimes U$ has analytically nilpotent curvature.

4.6. Homotopy invariance of the $\mathrm{X}$-complex. In this section, we assume that the field $F$ has characteristic 0 . This is needed to prove that homotopic homomorphisms defined on a quasi-free algebra induce chain homotopic maps between the $X$-complexes. If we understand homotopy to mean "polynomial homotopy", then this is already shown by Cuntz and Quillen (see 17, Sections 7-8]). In the context of complete bornological $V$-algebras, the proof for polynomial homotopies still works for dagger homotopies. The corresponding statement for the $B, b$-bicomplexes is 11. Proposition 4.3.3]. For quasi-free algebras, the canonical projection from the $\bar{B}, b$-bicomplex to the $X$-complex is a chain homotopy equivalence. This implies the following:

Proposition 4.6.1. Let $R$ and $S$ be projective systems of complete bornological F-algebras. Let $f, g: R \rightrightarrows S$ be two homomorphisms that are dagger homotopic. 
Assume that $F$ has characteristic 0 and that $R$ is quasi-free. Then the induced chain maps $X(f), X(g): X(R) \rightrightarrows X(S)$ are chain homotopic.

Proof. It suffices to treat an elementary dagger homotopy. Define

$$
\begin{aligned}
\eta_{n}: \Omega^{n}(S \otimes V[t]) \otimes F & \rightarrow \Omega^{n-1}(S) \otimes F, \\
a_{0} \mathrm{~d} a_{1} \ldots \mathrm{d} a_{n} & \mapsto \int_{0}^{1} a_{0}(t) \frac{\partial a_{1}(t)}{\partial t} \mathrm{~d} a_{2}(t) \ldots \mathrm{d} a_{n}(t) \mathrm{d} t,
\end{aligned}
$$

for $n=1,2$. Here integration and differentiation are defined formally by rescaling the coefficients of polynomials $a_{i} \in S \otimes F[t]$. We claim that $\eta_{n}$ extends to a bounded linear map $\eta_{n}: \Omega^{n}\left(S \otimes V[t]^{\dagger}\right) \otimes F \rightarrow \Omega^{n-1}(S) \otimes F$. To see this, let $T:=S \otimes V[t]_{\mathrm{lg}}$. Then $\Omega^{n}(T) \cong T^{+} \otimes T^{\otimes n} \cong T^{\otimes n} \oplus T^{\otimes n+1}$. So it suffices to show that $\eta_{n}$ is bounded on $T^{\otimes n} \otimes F \cong S^{\otimes n} \otimes V[t]_{\mathrm{lg}}^{\otimes n} \otimes F$. This follows if the map

$$
\begin{aligned}
V[t]_{\lg }^{\otimes n} \otimes F & \rightarrow F \\
a_{0} \otimes a_{1} \otimes \cdots \otimes a_{n} & \mapsto \int_{0}^{1} a_{0}(t) \frac{\partial a_{1}(t)}{\partial t} \cdot a_{2}(t) \cdots a_{n}(t) \mathrm{d} t
\end{aligned}
$$

is bounded. The formal differentiation on $V[t]_{\lg }$ is clearly bounded. And $V[t]_{\lg }$ is a bornological algebra. So this happens if and only if the integration map

$$
V[t]_{\lg } \otimes F \rightarrow F, \quad a(t)=\sum_{l=0}^{\infty} c_{l} t^{l} \mapsto \sum_{l=0}^{\infty} \frac{c_{l}}{l+1}
$$

is bounded. If $\mathbb{F}$ has characteristic 0 , then $l+1$ is invertible in $V$ for all $l \in \mathbb{N}$. If $\mathbb{F}$ has finite characteristic $p$, then the valuation of $l+1$ grows at most logarithmically. In any case, this is dominated by the linear growth of the exponents of $\pi$ for a subset of linear growth in $V[t]$. Thus the integration map above is bounded, and then so are the maps $\eta_{n}$. We still write $\eta_{n}$ for their unique bounded extensions to the completions.

Let $\eta_{0}=0$. Then $[\eta, b]=0$. Therefore, $\eta_{2}\left(b\left(\widetilde{\Omega}^{3}\left(S \otimes V[t]^{\dagger}\right)\right)\right) \subseteq b\left(\bar{\Omega}^{2}(S)\right)$. So $\eta$ defines a map $X^{(2)}\left(S \bar{\otimes} V[t]^{\dagger}\right) \rightarrow X(S)$, where $X^{(2)}$ is the truncated $B$-b-complex defined in [26 Definition A.122].

Let $\xi_{2}: X^{(2)}\left(S \Phi V[t]^{\dagger}\right) \rightarrow X\left(S \otimes V[t]^{\dagger}\right)$ be the canonical projection. Then

$$
[\eta, B+b]=\left(X\left(\mathrm{ev}_{1}\right)-X\left(\mathrm{ev}_{0}\right)\right) \circ \xi: X^{(2)}\left(S \otimes V[t]^{\dagger}\right) \rightarrow X(S) .
$$

Now let $H: R \rightarrow S \otimes V[t]^{\dagger}$ be an elementary dagger homotopy between $f$ and $g$. Then $\eta \circ X^{(2)}(H): X^{(2)}(R) \rightarrow X(S)$ is a chain homotopy between $X(f) \circ \xi_{2}$ and $X(g) \circ \xi_{2}$, where $\xi_{2}: X^{(2)}(R) \rightarrow X(R)$ is the canonical projection. Since $R$ is analytically quasi-free, it is in particular quasi-free, so that $\xi_{2}$ is a chain homotopy equivalence. Let $\alpha: X(R) \rightarrow X^{(2)}(R)$ be the homotopy inverse of $\xi_{2}$. Then $\eta \circ \alpha$ is the desired chain homotopy between $X(f)$ and $X(g)$.

TheOREm 4.6.2. Let $A$ and $B$ be pro-algebras. If two homomorphisms $f_{0}, f_{1}: A \rightrightarrows$ $B$ are dagger homotopic, then they induce homotopic chain maps $\mathbb{H} \mathbb{A}(A) \rightarrow \mathbb{H} \mathbb{A}(B)$. And then $\mathrm{HA}_{*}\left(f_{0}\right)=\mathrm{HA}_{*}\left(f_{1}\right)$.

Proof. The homomorphisms $\mathcal{T} f_{0}, \mathcal{T} f_{1}: \mathcal{T} A \rightrightarrows \mathcal{T} B$ lift $f_{0}$ and $f_{1}$. Since $\mathcal{T} A$ is analytically quasi-free and $\mathcal{J} B$ is analytically nilpotent, Proposition 4.3 .10 provides a dagger homotopy between $\mathcal{T} f_{0}$ and $\mathcal{T} f_{1}$. Then the chain maps $X(\mathcal{T} A \otimes F) \rightrightarrows$ $X(\mathcal{T} B \otimes F)$ induced by $f_{0}$ and $f_{1}$ are chain homotopic by Proposition 4.6.1 This remains so on the homotopy projective limits. And then $f_{0}$ and $f_{1}$ induce the 
same map on the homology of the homotopy projective limits. That is, $\operatorname{HA}_{*}\left(f_{0}\right)=$ $\mathrm{HA}_{*}\left(f_{1}\right)$.

4.7. Invariance under analytically nilpotent extensions. We continue to assume that $F$ has characteristic 0 .

THEOREM 4.7.1. Let $J \hookrightarrow E \stackrel{p}{\rightarrow} A$ be a semi-split, analytically nilpotent extension of pro-algebras. Then $p$ induces a chain homotopy equivalence $\mathbb{H} \mathbb{A}(E) \simeq \mathbb{H} \mathbb{A}(A)$ and $\mathbb{H} \mathbb{A}(J)$ is contractible. So $\mathrm{HA}_{*}(E) \cong \mathrm{HA}_{*}(A)$ and $\mathrm{HA}_{*}(J)=0$. If $E$ is analytically quasi-free, then $\mathbb{H} \mathbb{A}(A)$ is chain homotopy equivalent to $X(E \otimes F)$ and $\mathrm{HA}_{*}(A)$ is isomorphic to the homology of the homotopy projective limit of $X(E \otimes F)$.

Proof. The composite map $\mathcal{T} E \rightarrow E \rightarrow A$ is pro-algebra homomorphism with a pro-linear section. Its kernel $K$ is an extension of $\mathcal{J} E$ by $J$ and hence analytically nilpotent by Proposition 4.3.13 Both $\mathcal{T} E$ and $\mathcal{T} A$ are analytically quasi-free by Proposition 4.4.6. Proposition 4.3.10 applied to the extensions $K \nrightarrow \mathcal{T} E \rightarrow A$ and $\mathcal{J} A \nrightarrow \mathcal{T} A \rightarrow A$ shows that $\mathcal{T} A$ and $\mathcal{T} E$ are dagger homotopy equivalent. This together with Proposition 4.6.1 implies that $\mathbb{H} \mathbb{A}(A)=X(\mathcal{T} A \otimes F)$ and $\mathbb{H} \mathbb{A}(E)=$ $X(\mathcal{T} E \otimes F)$ are homotopy equivalent. This remains so for their homotopy projective limits. So $\mathrm{HA}_{*}(E) \cong \mathrm{HA}_{*}(A)$. More precisely, the isomorphism is the map induced by the quotient map $E \rightarrow A$.

Since $J$ and $\mathcal{J} J$ are analytically nilpotent, so is $\mathcal{T} J$ by Proposition 4.3 .13 . Since $\mathcal{T} J$ is analytically quasi-free, Proposition 4.3 .10 may be applied to the extensions $\mathcal{T} J=\mathcal{T} J \rightarrow 0$ and $0=0=0$ of 0 . Thus $\mathcal{T} J$ is dagger homotopy equivalent to 0 . Then $\mathbb{H} \mathbb{A}(J) \simeq 0$ and $\mathrm{HA}_{*}(J) \cong 0$.

Now assume $E$ to be analytically quasi-free. Then Proposition 4.3.10 shows that the extensions of $A$ by $\mathcal{T} A$ and $E$ are dagger homotopy equivalent. Then $X(E) \otimes F$ is homotopy equivalent to $X(\mathcal{T} A) \otimes F$. Then $\mathbb{H} \mathbb{A}(A)$ is homotopy equivalent to the homotopy projective limit of the projective system of chain complexes $X(E) \otimes F$.

Corollary 4.7.2. Let $A$ be an analytically quasi-free algebra. Then $\mathbb{H} \mathbb{A}(A)$ is chain homotopy equivalent to $X(A \otimes F)$ and $\mathrm{HA}_{*}(A)$ is isomorphic to the homology of $X(A \otimes F)$.

Proof. Theorem 4.7.1 shows that $\mathbb{H} \mathbb{A}(A)$ is homotopy equivalent to $X(A \otimes F)$. Then $\mathrm{HA}_{*}(A)$ is isomorphic to the homology of holim $X(A \otimes F)$. Since $X(A \otimes F)$ is a constant projective system, it is chain homotopy equivalent to its homotopy projective limit. So we simply get the ordinary homology of $X(A \otimes F)$.

COROLlary 4.7.3. $\mathbb{H} \mathbb{A}(V)$ is homotopy equivalent to $F$ with zero boundary map.

Proof. The algebra $V$ is analytically quasi-free by Proposition 4.3.6 Then $\mathbb{H} \mathbb{A}(V) \simeq X(F)$ by Corollary 4.7.2 A small calculation shows that any element of $\Omega^{1}(V)$ is a commutator. So $X(F)$ is $F$ with zero boundary map.

\section{Excision}

The goal of this section is to prove the following excision theorem for analytic cyclic homology: 
THEOREM 5.1. Let $K \stackrel{i}{\rightarrow} E \stackrel{p}{\rightarrow} Q$ be a semi-split extension of pro-algebras with a pro-linear section $s: Q \rightarrow E$. Then there is a natural exact triangle

$$
\mathbb{H} \mathbb{A}(K) \stackrel{i_{*}}{\rightarrow} \mathbb{H} \mathbb{A}(E) \stackrel{p_{*}}{\rightarrow} \mathbb{H} \mathbb{A}(Q) \stackrel{\delta}{\rightarrow} \mathbb{H} \mathbb{A}(K)[-1]
$$

in the homotopy category of chain complexes of projective systems of bornological $V$-modules. Thus there is a natural long exact sequence

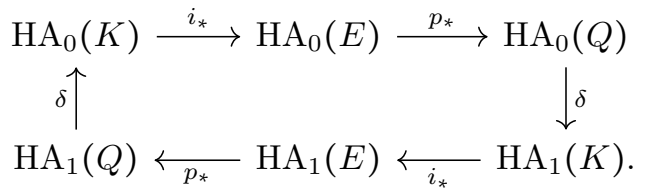

Here the arrows in the "homotopy category" are chain homotopy classes of chain maps. This homotopy category is triangulated over any additive category, with triangles coming from mapping cones of chain maps.

The proof will take up the rest of this section. It follows 25,26 . We use the left ideal $\mathcal{L}$ in $\mathcal{T} E$ generated by $K$ and prove chain homotopy equivalences $X(\mathcal{T} K) \simeq X(\mathcal{L})$ and $X(\mathcal{L}) \simeq X(\mathcal{T} E: \mathcal{T} Q)$ as chain complexes in the additive category of projective systems of bornological $V$-modules. First, the pro-linear section $s$ yields two bounded maps $s_{L}, s_{R}: \Omega^{\mathrm{ev}} Q \rightrightarrows \Omega^{\mathrm{ev}} E$ defined by

$$
\begin{gathered}
s_{L}\left(q_{0} \mathrm{~d} q_{1} \ldots \mathrm{d} q_{2 n}\right):=s\left(q_{0}\right) \mathrm{d} s\left(q_{1}\right) \ldots \mathrm{d} s\left(q_{2 n}\right), \\
s_{R}\left(\mathrm{~d} q_{1} \ldots \mathrm{d} q_{2 n} q_{2 n+1}\right)
\end{gathered}
$$

for all $q_{0}, q_{2 n+1} \in Q^{+}$and $q_{i} \in Q$ for $1 \leq i \leq 2 n$. Let $m \in \mathbb{N}^{*}$. Both $s_{L}$ and $s_{R}$ map J $Q^{m j}$ to $\mathrm{J} E^{m j}$ for all $j \in \mathbb{N}$ by 4.4 .8 . Thus they induce bounded linear maps on the tubes, from $\mathcal{U}\left(\mathrm{T} Q, \mathrm{~J} Q^{m}\right)$ to $\mathcal{U}\left(\mathrm{T} E, \mathrm{~J} E^{m}\right)$. Both are sections for the canonical projection $\mathcal{U}\left(\mathrm{T} E, \mathrm{~J} E^{m}\right) \rightarrow \mathcal{U}\left(\mathrm{T} Q, \mathrm{~J} Q^{m}\right)$. These sections remain bounded for the linear growth bornologies relative to $\mathcal{U}\left(\mathrm{J} E, \mathrm{~J} E^{m}\right)$ and $\mathcal{U}\left(\mathrm{J} Q, \mathrm{~J} Q^{m}\right)$ by Proposition 4.4.11. Thus they extend to bounded $V$-module maps on the completions. These maps for all $m \in \mathbb{N}^{*}$ form two pro-linear sections for $\mathcal{T} p: \mathcal{T} E \rightarrow \mathcal{T} Q$. They induce two sections for the canonical chain map $X(\mathcal{T} p): X(\mathcal{T} E) \rightarrow X(\mathcal{T} Q)$. Let

$$
X(\mathcal{T} E: \mathcal{T} Q):=\operatorname{ker}(X(\mathcal{T} p): X(\mathcal{T} E) \rightarrow X(\mathcal{T} Q)) .
$$

There is a semi-split extension of chain complexes

$$
X(\mathcal{T} E: \mathcal{T} Q) \nrightarrow \mathcal{T} E \rightarrow \mathcal{T} Q .
$$

Since $X(\mathcal{T} p) \circ X(\mathcal{T} i)=X(\mathcal{T}(p \circ i))=0$, the chain map $X(\mathcal{T} i)$ factors through $X(\mathcal{T} E: \mathcal{T} Q)$. We are going to prove that this chain map $X(\mathcal{T} K) \rightarrow X(\mathcal{T} E: \mathcal{T} Q)$ is a chain homotopy equivalence. Then the homotopy projective limit of $X(\mathcal{T} K)$ is homotopy equivalent to that of $X(\mathcal{T} E: \mathcal{T} Q)$, and the latter fits into a semi-split extension of chain complexes with the homotopy projective limits of $X(\mathcal{T} E)$ and $X(\mathcal{T} Q)$. As a result, Theorem 5.1 follows if the inclusion map $X(\mathcal{T} K) \rightarrow X(\mathcal{T} E$ : $\mathcal{T} Q)$ is a chain homotopy equivalence.

Our construction of the chain homotopy equivalence will, in principle, be explicit and natural, using only the multiplication maps in our pro-algebras and the prolinear sections $s_{L}$ and $s_{R}$ above. Therefore, we assume for simplicity from now on that we are dealing with an extension of (complete, torsion-free bornological) algebras $K \nrightarrow E \rightarrow Q$. In general, we may rewrite the semi-split extension above as a projective system of semi-split algebra extensions $K_{n} \nrightarrow E_{n} \rightarrow Q_{n}$ with compatible 
bounded linear sections; this uses arguments as in the proof of Proposition 4.3.13. To simplify notation, we write down the proof below only for a semi-split algebra extension. The chain maps and homotopies that we are going to build for the extensions $K_{n} \nrightarrow E_{n} \rightarrow Q_{n}$ form morphisms of projective systems. So the same proof works for a semi-split extension of pro-algebras.

5.1. The pro-algebra $\mathcal{L}$. In the following, we identify $\mathrm{T} E$ with $\Omega^{\mathrm{ev}} E$ and $E$ with $\Omega^{0}(E) \subseteq \Omega^{\mathrm{ev}} E$. So the map $\sigma_{E}: E \rightarrow \mathcal{T} E$ disappears from our notation. Proposition 4.4.6 gives an isomorphism of left $\mathcal{T} E$-modules

$$
(\mathcal{T} E)^{+} \otimes E \stackrel{\sim}{\rightarrow} \mathcal{T} E, \quad \omega \otimes x \mapsto \omega \odot x .
$$

Explicitly, the inverse of this isomorphism is given by

$$
\omega \mathrm{d} e_{2 n-1} \mathrm{~d} e_{2 n} \mapsto \omega \otimes\left(e_{2 n-1} \cdot e_{2 n}\right)-\left(\omega \odot e_{2 n-1}\right) \otimes e_{2 n} .
$$

These two maps also define an isomorphism for the purely algebraic tensor algebras:

$$
(\mathrm{T} E)^{+} \otimes E \stackrel{\sim}{\rightarrow} \mathrm{T} E, \quad \omega \otimes e \mapsto \omega \odot e .
$$

Variants of this isomorphism and the following ones were proven already in $\mathbf{2 6}$, Section 4.3.2]. Let $L \subseteq \mathrm{T} E$ be the left ideal generated by $K$. The bounded linear section $s: Q \rightarrow E$ yields an isomorphism of bornological $V$-modules $E \cong K \oplus Q$. Then 5.1 .3 implies an isomorphism

$$
(\mathrm{T} E)^{+} \otimes K \stackrel{\simeq}{\rightarrow} L, \quad \omega \otimes k \mapsto \omega \odot k .
$$

The explicit formula for the isomorphism in 5.1.2 and its inverse imply

$$
L=K \oplus \bigoplus_{n \geq 1} \Omega^{2 n-1}(E) \mathrm{d} K
$$

as in the proof of [26 Lemma 4.55]. Let $I:=\operatorname{ker}(\mathrm{T} p: \mathrm{T} E \rightarrow \mathrm{T} Q)$. This is part of semi-split extensions

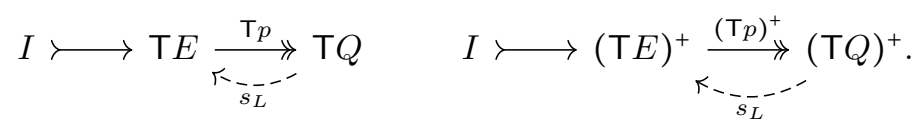

LEMMA 5.1.6. The following maps are isomorphisms:

$$
\begin{array}{rlrl}
\Psi: L^{+} \otimes(\mathrm{T} Q)^{+} \stackrel{\simeq}{\rightarrow}(\mathrm{T} E)^{+}, & l \otimes \eta \mapsto l \odot s_{L}(\eta), \\
L \otimes(\mathrm{T} Q)^{+} \stackrel{\simeq}{\rightarrow} I, & l \otimes \eta \mapsto l \odot s_{L}(\eta), \\
(\mathrm{T} E)^{+} \otimes K \otimes(\mathrm{T} Q)^{+} \stackrel{\simeq}{\rightarrow} I, & \omega \otimes k \otimes \eta \mapsto \omega \odot k \odot s_{L}(\eta), \\
(\mathrm{T} Q)^{+} \otimes K \otimes(\mathrm{T} E)^{+} \stackrel{\simeq}{\rightarrow} I, & \eta \otimes k \otimes \omega \mapsto s_{R}(\eta) \odot k \odot \omega, \\
(\mathrm{T} Q)^{+} \otimes K \otimes L^{+} \stackrel{\simeq}{\rightarrow} L, & \eta \otimes k \otimes l & \mapsto s_{R}(\eta) \odot k \odot l .
\end{array}
$$

Proof. The computations in [26, Section 4.3.1] show this. We briefly sketch them. The isomorphisms (5.1.7) and (5.1.8) are equivalent because of the semi-split extension (5.1.5). And (5.1.8) and (5.1.9) are equivalent because of the isomorphism 5.1.4). The isomorphisms (5.1.9) and 5.1.10 imply each other by taking opposite algebras because this reverses the order of multiplication and exchanges $s_{L}$ and $s_{R}$. And 5.1.10 implies 5.1.11) by substituting $(\mathrm{T} E)^{+} \cong L^{+} \otimes(\mathrm{T} Q)^{+}$and $I \cong L \otimes(\mathrm{T} Q)^{+}$in (5.1.11) and then cancelling the factor $(\mathrm{T} Q)^{+}$on both sides.

So it suffices to prove that $\Psi$ is an isomorphism. We describe its inverse $\Psi^{-1}$. Split a differential form $e_{0} \mathrm{~d} e_{1} \ldots \mathrm{d} e_{2 n} \in \Omega^{2 n} E$ so that each coefficient $e_{j}$ belongs 
either to $K$ or $s(Q)$, or is 1 in case of $e_{0}$; this is possible because of the direct sum decomposition $E \cong K \oplus s(Q)$; write $k_{i}:=e_{i}$ or $q_{i}:=s^{-1}\left(e_{i}\right)$ accordingly. If no $e_{i}$ belongs to $K$, then

$$
\Psi^{-1}\left(s\left(q_{0}\right) \mathrm{d} s\left(q_{1}\right) \ldots \mathrm{d} s\left(q_{2 n}\right)\right)=1 \otimes q_{0} \mathrm{~d} q_{1} \ldots \mathrm{d} q_{2 n} .
$$

Otherwise, there is a largest $i \leq 2 n$ with $e_{i} \in K$. If $i=0$, then

$$
\Psi^{-1}\left(k_{0} \mathrm{~d} s\left(q_{1}\right) \ldots \mathrm{d} s\left(q_{2 n}\right)\right)=k_{0} \otimes \mathrm{d} q_{1} \ldots \mathrm{d} q_{2 n} .
$$

If $i$ is even and non-zero, then

$$
\Psi^{-1}\left(e_{0} \mathrm{~d} e_{1} \ldots \mathrm{d} e_{i-1} \mathrm{~d} k_{i} \mathrm{~d} s\left(q_{i+1}\right) \ldots \mathrm{d} s\left(q_{2 n}\right)\right)=e_{0} \mathrm{~d} e_{1} \ldots \mathrm{d} e_{i-1} \mathrm{~d} k_{i} \otimes \mathrm{d} q_{i+1} \ldots \mathrm{d} q_{2 n} .
$$

If $i$ is odd, then

$$
\begin{aligned}
\Psi^{-1}\left(e_{0} \mathrm{~d} e_{1} \ldots \mathrm{d} e_{i-1} \mathrm{~d} k_{i} \mathrm{~d} s\left(q_{i+1}\right)\right. & \left.\ldots \mathrm{d} s\left(q_{2 n}\right)\right) \\
= & e_{0} \mathrm{~d} e_{1} \ldots \mathrm{d} e_{i-1} \odot\left(k_{i} \cdot s\left(q_{i+1}\right)\right) \otimes \mathrm{d} q_{i+2} \ldots \mathrm{d} q_{2 n} \\
& \quad-e_{0} \mathrm{~d} e_{1} \ldots \mathrm{d} e_{i-1} \odot k_{i} \otimes q_{i+1} \mathrm{~d} q_{i+2} \ldots \mathrm{d} q_{2 n} .
\end{aligned}
$$

A direct computation using $\mathrm{d} k_{i} \mathrm{~d} s\left(q_{i+1}\right)=k_{i} \cdot s\left(q_{i+1}\right)-k_{i} \odot s\left(q_{i+1}\right)$ shows that

$$
\Psi \circ \Psi^{-1}\left(e_{0} \mathrm{~d} e_{1} \ldots \mathrm{d} e_{2 n}\right)=e_{0} \mathrm{~d} e_{1} \ldots \mathrm{d} e_{2 n}
$$

for all $e_{0} \in\{1\} \cup K \cup s(Q), e_{1}, \ldots, e_{n} \in K \cup s(Q)$. Then one shows that the map $\Psi^{-1}$ is surjective: its image contains all elements of the form $1 \otimes \eta$ for $\eta \in(\mathrm{T} Q)^{+}$and $\omega \otimes \mathrm{d} q_{1} \ldots \mathrm{d} q_{2 n}$ with $\omega \in L^{+}$by the first two cases with no $i$ or even $i$, respectively. And modulo a term of this form, the image of $\Psi^{-1}$ contains all $\omega \odot k \otimes q_{0} \mathrm{~d} q_{1} \ldots \mathrm{d} q_{2 n}$ with $\omega \in(\mathrm{T} E)^{+}, k \in K$ because of the formula in the case of odd $i$. This exhausts $L^{+} \otimes(\mathrm{T} Q)^{+}$because of the isomorphism (5.1.4.

We are going to pass to the analytic tensor algebras and describe "analytic" analogues of $L, I \subseteq \mathrm{T} E$ and of the isomorphisms and semi-split extensions above. For $m \in \mathbb{N}^{*}$, let

$$
\begin{aligned}
I_{(m)} & :=\operatorname{ker}\left(\mathcal{U}\left(\mathrm{T} E, \mathrm{~J} E^{m}\right) \rightarrow \mathcal{U}\left(\mathrm{T} Q, \mathrm{~J} Q^{m}\right)\right), \\
L_{(m)} & :=K \oplus \bigoplus_{n \geq 1} \pi^{-\lfloor n / m\rfloor} \cdot \Omega^{2 n-1}(E) \mathrm{d} K .
\end{aligned}
$$

It is easy to see that $I_{(m)}$ is a two-sided and $L_{(m)}$ a left ideal in $\mathcal{U}\left(\mathrm{T} E, \mathrm{~J} E^{\infty}\right)$. In particular, both are $V$-algebras in their own right. Inspection shows that

$$
I_{(m)}=\mathcal{U}\left(\mathrm{T} E, \mathrm{~J} E^{m}\right) \cap(I \otimes F), \quad L_{(m)}=\mathcal{U}\left(\mathrm{T} E, \mathrm{~J} E^{m}\right) \cap(L \otimes F)
$$

as $V$-submodules of $\mathrm{T} E \otimes F$. The maps in the projective system $\mathcal{U}\left(\mathrm{T} E, \mathrm{~J} E^{\infty}\right)$ make $\left(I_{(m)}\right)_{m \in \mathbb{N}^{*}}$ and $\left(L_{(m)}\right)_{m \in \mathbb{N}^{*}}$ projective systems by restriction. We equip each $\mathcal{U}\left(\mathrm{T} E, \mathrm{~J} E^{m}\right)$ with the bornology $\mathcal{B}_{m}$ described in Corollary 4.4.14 using the linear growth bornology instead would slightly complicate the estimates below. We give $I_{(m)}$ and $L_{(m)}$ the subspace bornologies. So the bornology on $L_{(m)}$ is cofinally generated by

$$
(M \cap K) \oplus \bigoplus_{n=1}^{\infty} \pi^{-\lfloor n / m\rfloor} \Omega^{2 n-1} M \mathrm{~d}(M \cap K)
$$

for bounded $V$-submodules $M \subseteq E$. Let $\mathcal{I}:=\left(\widetilde{I_{(m)}}\right)_{m \in \mathbb{N}^{*}}$ and $\mathcal{L}:=\left(\widetilde{L_{(m)}}\right)_{m \in \mathbb{N}^{*}}$ be the projective systems formed by the completions.

Since $\mathcal{U}\left(\mathrm{T} E, \mathrm{~J} E^{m}\right)$ is a subalgebra of $\mathrm{T} E \otimes F$ and the maps in 5.1 .3 , 5.1.4 and $5.1 .7-5.1 .11$ only involve Fedosov products and the maps $s_{L}$ and $s_{R}, 5.1 .12$ 
implies that these maps still exist and are bounded if $\mathrm{T} E, \mathrm{~T} Q, I, L$ are replaced by $\mathcal{U}\left(\mathrm{T} E, \mathrm{~J} E^{m}\right), \mathcal{U}\left(\mathrm{T} Q, \mathrm{~J} Q^{m}\right), I_{(m)}, L_{(m)}$, respectively, each equipped with the bornologies specified above. The inverse maps for these isomorphisms are slightly more complicated, however: they may shift the index $m$ in the projective system:

Lemma 5.1.14. The inverses to the isomorphisms above extend to bounded maps

$$
\begin{aligned}
\mathcal{U}\left(\mathrm{T} E, \mathrm{~J} E^{m+1}\right) & \rightarrow \mathcal{U}\left(\mathrm{T} E, \mathrm{~J} E^{m}\right)^{+} \otimes E, \\
L_{(m+1)} & \rightarrow \mathcal{U}\left(\mathrm{T} E, \mathrm{~J} E^{m}\right)^{+} \otimes K, \\
\mathcal{U}\left(\mathrm{T} E, \mathrm{~J} E^{2 m}\right)^{+} & \rightarrow L_{(m)}^{+} \otimes \mathcal{U}\left(\mathrm{T} Q, \mathrm{~J} Q^{m}\right)^{+}, \\
I_{(2 m)} & \rightarrow L_{(m)} \otimes \mathcal{U}\left(\mathrm{T} Q, \mathrm{~J} Q^{m}\right)^{+}, \\
I_{(2 m)} & \rightarrow \mathcal{U}\left(\mathrm{T} E, \mathrm{~J} E^{m}\right)^{+} \otimes K \otimes \mathcal{U}\left(\mathrm{T} Q, \mathrm{~J} Q^{m}\right)^{+}, \\
I_{(2 m)} & \rightarrow \mathcal{U}\left(\mathrm{T} Q, \mathrm{~J} Q^{m}\right)^{+} \otimes K \otimes \mathcal{U}\left(\mathrm{T} E, \mathrm{~J} E^{m}\right)^{+}, \\
L_{(2 m)} & \rightarrow \mathcal{U}\left(\mathrm{T} Q, \mathrm{~J} Q^{m}\right)^{+} \otimes K \otimes L_{(m)}^{+} .
\end{aligned}
$$

Proof. Our explicit formula for the first map shows that it reduces the total degree of a differential form by at most 2. Since $\frac{n+1}{m+1} \leq \frac{n}{m}$ for all $n \geq m$ and $\left\lfloor\frac{n+1}{m+1}\right\rfloor=$ $\left\lfloor\frac{n}{m}\right\rfloor=0$ if $n<m$, it follows that it defines a map $\mathcal{U}\left(\mathrm{T} E, \mathrm{~J} E^{m+1}\right) \rightarrow \mathcal{U}\left(\mathrm{T} E, \mathrm{~J} E^{m}\right)^{+} \otimes E$ that is bounded for the bornologies described in Corollary 4.4.14 The second map is a restriction of the first map, so that it is covered by the same argument.

Our explicit formula for the third map shows that it maps a differential form of degree $2 n$ to a sum of tensor products involving differential forms of degree $2 j$ and $2(n-j-1)$ or $2(n-j)$; in the first case, $j<n$ and the differential form in $L$ is already explicitly written as $\omega \odot k$, so that the isomorphism $L \rightarrow(\mathrm{T} E)^{+} \otimes K$ does not reduce the degree any further. This shows that the same degree estimate applies to the fourth map in the lemma. The fifth map differs from that only by taking opposite algebras, and the sixth map is a restriction of the fifth one. This is why the following estimates cover all these maps at the same time.

That these maps are well defined between the relevant tube algebras amounts to the estimate $\lfloor n / 2 m\rfloor \leq\lfloor j / m\rfloor+\lfloor(n-j-1) / m\rfloor$ for all $n \in \mathbb{N}, 0 \leq j<n$. This is trivial for $n<2 m$, so that we assume $n \geq 2 m$. For fixed $n$, the right hand side is minimal if $j=m-1$, and then the needed estimate simplifies to $\lfloor n / 2 m\rfloor \leq\lfloor(n-m) / m\rfloor$. This is true for $2 m \leq n<4 m$. Since adding $2 m$ to $n$ increases $\lfloor n / 2 m\rfloor$ by 1 and $\lfloor(n-m) / m\rfloor$ by 2 , the inequality follows for all $n \in \mathbb{N}$. Now it follows that the maps in the lemma are well defined and bounded for the bornologies described in Corollary 4.4.14.

The composite maps

$$
\begin{gathered}
\mathcal{U}\left(\mathrm{T} E, \mathrm{~J} E^{m+1}\right)^{+} \otimes E \rightarrow \mathcal{U}\left(\mathrm{T} E, \mathrm{~J} E^{m+1}\right) \rightarrow \mathcal{U}\left(\mathrm{T} E, \mathrm{~J} E^{m}\right)^{+} \otimes E \\
\mathcal{U}\left(\mathrm{T} E, \mathrm{~J} E^{m+1}\right) \rightarrow \mathcal{U}\left(\mathrm{T} E, \mathrm{~J} E^{m}\right)^{+} \otimes E \rightarrow \mathcal{U}\left(\mathrm{T} E, \mathrm{~J} E^{m}\right)^{+}
\end{gathered}
$$

are the structure maps in our projective systems because they extend the identity maps on $(\mathrm{T} E)^{+} \otimes E$ and $\mathrm{T} E$, respectively. Thus these two families of maps for $m \in \mathbb{N}^{*}$ are isomorphisms of projective systems of bornological $V$-modules that are inverse to each other. This remains so when we complete, giving an isomorphism $(\mathcal{T} E)^{+} \otimes E \stackrel{\simeq}{\rightarrow} \mathcal{T} E$. The same argument applies to the other isomorphisms above. Summing up, we get the following isomorphisms of projective systems of bornological 
$V$-modules:

$$
\begin{array}{rlrl}
(\mathcal{T} E)^{+} \otimes E & \stackrel{\simeq}{\rightarrow} \mathcal{T} E, & \omega \otimes e \mapsto \omega \odot e, \\
(\mathcal{T} E)^{+} \otimes K \stackrel{\simeq}{\rightarrow} \mathcal{L}, & \omega \otimes k \mapsto \omega \odot k, \\
\mathcal{L}^{+} \otimes(\mathcal{T} Q)^{+} \stackrel{\simeq}{\rightarrow}(\mathcal{T} E)^{+}, & l \otimes \eta \mapsto l \odot s_{\mathcal{L}}(\eta), \\
\mathcal{L} \otimes(\mathcal{T} Q)^{+} \stackrel{\simeq}{\rightarrow} \mathcal{I}, & l \otimes \eta \mapsto l \odot s_{L}(\eta), \\
(\mathcal{T} E)^{+} \otimes K \nabla(\mathcal{T} Q)^{+} \stackrel{\simeq}{\rightarrow} \mathcal{I}, & \omega \otimes k \otimes \eta \mapsto \omega \odot k \odot s_{L}(\eta), \\
(\mathcal{T} Q)^{+} \otimes K \nabla(\mathcal{T} E)^{+} \stackrel{\simeq}{\rightarrow} \mathcal{I}, & \eta \otimes k \otimes \omega \mapsto s_{R}(\eta) \odot k \odot \omega, \\
(\mathcal{T} Q)^{+} \otimes K \nabla \mathcal{L}^{+} \stackrel{\simeq}{\rightarrow} \mathcal{L}, & \eta \otimes k \otimes l \mapsto s_{R}(\eta) \odot k \odot l .
\end{array}
$$

In addition, there are semi-split extensions

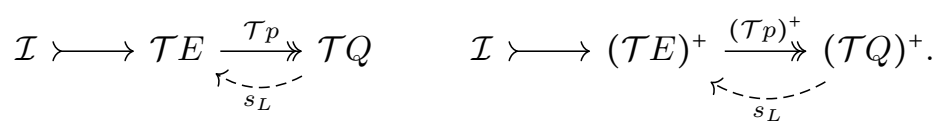

Here 5.1.15) is the same as 5.1.1). So it follows already from the analytic nilpotence machinery in Section 4 And (5.1.15) easily implies (5.1.16). The isomorphisms (5.1.18)-5.1.21) follow from (5.1.15)-55.1.17) and the semi-split extension (5.1.22) as in the proof of Lemma 5.1.6 It seems, however, that the existence of the maps $s_{L}, s_{R}: \mathcal{T} Q \rightrightarrows \mathcal{T} E$ and (5.1.17) do not follow from the machinery in Section 4 and must be checked by hand.

THEOREM 5.1.23. The chain map $X(\mathcal{L}) \rightarrow X(\mathcal{T} E: \mathcal{T} Q)$ induced by the inclusion $\mathcal{L} \hookrightarrow \mathcal{T} E$ is a chain homotopy equivalence.

Proof. The proofs of [26. Theorems 4.66 and 4.67] carry over literallly to our analytic tensor algebras, using the isomorphisms 5.1.15 - 5.1.21 and the semi-split extension 5.1.22). We merely have to replace the symbols $\otimes, A:=\overleftarrow{\mathrm{T}} E, \overleftarrow{\mathrm{T}} Q, \overleftarrow{L}$ $\overleftarrow{I}$ and $\overleftarrow{G}$ in that proof by $\nabla, \mathcal{T} E, \mathcal{T} Q, \mathcal{L}, \mathcal{I}$ and $(\mathcal{T} Q)^{+} \bar{\otimes} K$, respectively; and $\overleftarrow{\Omega}^{\text {ev }} E$ and $\overleftarrow{\Omega}^{\text {odd }} E$ in 26 become $\mathcal{T} E$ and $(\mathcal{T} E)^{+} \otimes E$, respectively, with the latter identified with differential forms of odd degree. [26. Theorem 5.80] is a similar translation exercise for the analytic cyclic homology theory for bornological algebras over the complex numbers, and the situation in this article is quite similar.

We briefly sketch the main idea of the proof. Proposition 4.4.6 and the definition of $\bar{\Omega}^{1}(\mathcal{T} E)$ imply that there is a semi-split free $\mathcal{T} E$-bimodule resolution

$$
\bar{\Omega}^{1}(\mathcal{T} E) \nrightarrow(\mathcal{T} E)^{+} \otimes(\mathcal{T} E)^{+} \rightarrow(\mathcal{T} E)^{+}
$$

with a natural pro-linear section $(\mathcal{T} E)^{+} \rightarrow(\mathcal{T} E)^{+} \otimes(\mathcal{T} E)^{+}, x \mapsto 1 \otimes x$. Let

$$
\begin{aligned}
& P_{0}:=\mathcal{L}^{+} \otimes \mathcal{L}^{+}+(\mathcal{T} E)^{+} \otimes \mathcal{L} \subseteq(\mathcal{T} E)^{+} \otimes(\mathcal{T} E)^{+}, \\
& P_{1}:=(\mathcal{T} E)^{+} D \mathcal{L} \subseteq \Omega^{1}(\mathcal{T} E)^{+} .
\end{aligned}
$$

This together with $\mathcal{L}^{+} \subseteq(\mathcal{T} E)^{+}$gives a subcomplex of the resolution above, and the standard section above yields a contracting homotopy for it, making it a resolution. The bimodules $P_{0}$ and $P_{1}$ are free; this is where the isomorphisms above enter. So $P_{1} \nrightarrow P_{0} \rightarrow \mathcal{L}^{+}$is a free $\mathcal{L}$-bimodule resolution. Then $\mathcal{L}$ is quasi-free, and the $X$-complex computes its periodic cyclic homology. And the commutator quotient complex $P_{1} /\left[\mathcal{L}, P_{1}\right] \rightarrow P_{0} /\left[\mathcal{L}, P_{0}\right]$ computes the Hochschild homology of $\mathcal{L}$. These 
commutator quotients are computed explicitly and shown to compute the relative Hochschild homology for the quotient map $\mathcal{T} E \rightarrow \mathcal{T} Q$. And then the isomorphism on Hochschild homology implies an isomorphism in cyclic homology and thus periodic cyclic homology.

5.2. Analytic quasi-freeness of $\mathcal{L}$. The proof of the excision theorem is completed by the following theorem:

THEOREM 5.2.1. There is a semi-split, analytically nilpotent extension $\mathcal{J} E \cap \mathcal{L} \rightarrow$ $\mathcal{L} \rightarrow K$ and $\mathcal{L}$ is analytically quasi-free.

This theorem and Theorem 4.7.1 imply that $\mathbb{H} \mathbb{A}(K)$ is chain homotopy equivalent to the $X$-complex of $\mathcal{L}$. Theorem 5.1 .23 identifies this with the relative $X$-complex $X(\mathcal{T} E: \mathcal{T} Q)$. And this yields the excision theorem. So it only remains to prove Theorem 5.2.1.

The canonical projection $\mathcal{T} E \rightarrow E$ restricts to a semi-split projection $\mathcal{L} \rightarrow K$. Its kernel $\mathcal{J} E \cap \mathcal{L} \subseteq \mathcal{J} E$ is a projective system of closed subalgebras. These are complete and torsion-free by [27, Theorem 2.3 and Lemma 4.2]; and subalgebras also clearly inherit the property of being semi-dagger. So $\mathcal{J} E \cap \mathcal{L}$ is a projective system of dagger algebras. Proposition 4.2.6 implies that it is again nilpotent mod $\pi$ because $\mathcal{J} E /(\mathcal{J} E \cap \mathcal{L})$ is torsion-free.

The proof of Theorem 5.1 .23 already shows that $\mathcal{L}$ is quasi-free. We need it to be analytically quasi-free, however. This is the main difficulty in Theorem 5.2.1. The proof of this uses the same ideas as the proof of the corresponding statement for analytic cyclic homology for bornological algebras over $\mathbb{C}$ in $[\mathbf{2 6}$. First, we define a homomorphism $v: L \rightarrow \mathrm{T} L$ for the purely algebraic version $L$ of $\mathcal{L}$. Then we show that this homomorphism extends uniquely to a homomorphism of pro-algebras $\mathcal{L} \rightarrow \mathcal{T} \mathcal{L}$ that is a section for the canonical projection $\mathcal{T} \mathcal{L} \rightarrow \mathcal{L}$.

We need some notation for elements of $T L$ and a certain grading on $T L$. Elements of $\mathrm{T} L$ are sums of differential forms $l_{0} \mathrm{D} l_{1} \ldots \mathrm{D} l_{2 n}$ with $l_{0} \in L^{+}, l_{1}, \ldots, l_{2 n} \in L$. We write $\odot$ for the Fedosov product in $\Omega^{\mathrm{ev}} L$ to distinguish it from the Fedosov product $\odot$ in $L$ and the resulting usual multiplication on $\Omega L$. Call an element of $\mathrm{T} L$ elementary if it is of the form $l_{0} \mathrm{D} l_{1} \ldots \mathrm{D} l_{2 n}$ with $l_{j}=e_{j, 0} \mathrm{~d} e_{j, 1} \ldots \mathrm{d} e_{j, 2 i_{j}}$ for $0 \leq j \leq 2 n$, and $e_{j, k} \in K \cup s(Q)$ for all occurring indices $j, k$, except that we allow $e_{j, 0}=1$ if $i_{j} \geq 1$ and $l_{0}=1$ if $i_{0}=0$; here $e_{j, 2 i_{j}} \in K$ because $l_{j} \in L$. Any element of $\mathrm{T} L$ is a finite linear combination of such elementary elements. The entries of an elementary element $\xi$ are the elements $e_{j, l} \in E$; its internal degree is $\operatorname{deg}_{i}(\xi)=\sum_{j=0}^{2 n} 2 i_{j}$; its external degree is $\operatorname{deg}_{e}(\xi)=6 n$ if $l_{0} \in L$ and $\operatorname{deg}_{e}=6 n-4$ if $l_{0}=1$, and the total degree $\operatorname{deg}_{t}(\xi)$ is the sum of these two degrees; this particular total degree already appears in the proof of [26, Lemma 5.102].

The definition of $v$ is based on the isomorphism $L \cong(\mathrm{T} E)^{+} \otimes K$ in (5.1.4). The restriction of $v$ to $K=\left(\Omega^{0} \mathrm{~T} E \cap L\right) \subseteq L$ is the obvious inclusion of $K$ into T $L$. We extend this map to $L$ using a homomorphism from TE to the algebra of $V$-module homomorphisms $\mathrm{T} L \rightarrow \mathrm{T} L$. Such a homomorphism is equivalent to a linear map $E \rightarrow \operatorname{Hom}(\mathrm{T} L, \mathrm{~T} L)$, which is, in turn, equivalent to a $V$-bilinear map $E \times \mathrm{T} L \rightarrow \mathrm{T} L$, which we denote as an operation $(e, \xi) \mapsto e \triangleright \xi$ for $e \in E, \xi \in \mathrm{T} L$. As in $[\mathbf{2 6}$, we first define the map $\nabla: L \rightarrow \Omega^{1}(L)$ by $\nabla\left(s_{R}(\xi) \odot k \odot l\right):=s_{R}(\xi) \odot k \mathrm{D} l$ for all $\xi \in(\mathrm{T} Q)^{+}$, $k \in K, l \in L^{+}$, with the understanding that $\mathrm{D} 1=0$ if $l$ is the unit element of $L$; this 
uses the inverse of the isomorphism 5.1.11. Then we let

$$
\begin{aligned}
e \triangleright x_{0} \mathrm{D} x_{1} \ldots \mathrm{D} x_{2 n} & =e \odot x_{0} \mathrm{D} x_{1} \ldots \mathrm{D} x_{2 n}-\mathrm{D} \nabla\left(e \odot x_{0}\right) \mathrm{D} x_{1} \ldots \mathrm{D} x_{2 n}, \\
e \triangleright \mathrm{D} x_{1} \mathrm{D} x_{2} \ldots \mathrm{D} x_{2 n} & =\nabla\left(e \odot x_{1}\right) \mathrm{D} x_{2} \ldots \mathrm{D} x_{2 n} .
\end{aligned}
$$

The curvature of the corresponding map $E \rightarrow \operatorname{Hom}(\mathrm{T} L, \mathrm{~T} L)$ acts by the operation $\omega_{\triangleright}\left(e_{1}, e_{2}\right) \xi:=\left(e_{1} \cdot e_{2}\right) \triangleright \xi-e_{1} \triangleright\left(e_{2} \triangleright \xi\right)$. It is computed in [26. Equation (5.91)]:

$$
\begin{aligned}
\omega_{\triangleright}\left(e_{1}, e_{2}\right) l_{0} \mathrm{D} l_{1} \ldots \mathrm{D} l_{2 n}=( & \left.\mathrm{d} e_{1} \mathrm{~d} e_{2} \odot l_{0}\right) \mathrm{D} l_{1} \ldots \mathrm{D} l_{2 n} \\
& +\nabla\left(e_{1} \odot \nabla\left(e_{2} \odot l_{0}\right)\right) \mathrm{D} l_{1} \ldots \mathrm{D} l_{2 n} \\
& -\mathrm{D} \nabla\left(\mathrm{d} e_{1} \mathrm{~d} e_{2} \odot l_{0}\right) \mathrm{D} l_{1} \ldots \mathrm{D} l_{2 n}, \\
\omega_{\triangleright}\left(e_{1}, e_{2}\right) \mathrm{D} l_{1} \ldots \mathrm{D} l_{2 n}=\nabla & \left.\left(e_{1} \cdot e_{2}\right) \odot l_{1}\right) \mathrm{D} l_{2} \ldots \mathrm{D} l_{2 n} \\
& -e_{1} \odot \nabla\left(e_{2} \odot l_{1}\right) \mathrm{D} l_{2} \ldots \mathrm{D} l_{2 n} \\
& +\mathrm{D} \nabla\left(e_{1} \odot \nabla\left(e_{2} \odot l_{1}\right)\right) \mathrm{D} l_{2} \ldots \mathrm{D} l_{2 n} .
\end{aligned}
$$

Finally, we define

$$
v\left(e_{0} \mathrm{~d} e_{1} \ldots \mathrm{d} e_{2 n} \odot k\right):=e_{0} \triangleright\left(\omega_{\triangleright}\left(e_{1}, e_{2}\right) \circ \cdots \circ \omega_{\triangleright}\left(e_{2 n-1}, e_{2 n}\right)\right)(k) .
$$

LEMma 5.2.2. The map $v: L \rightarrow \mathrm{T} L$ is an algebra homomorphism, and $p \circ v=\mathrm{id}_{L}$ for the canonical projection $p: \mathrm{T} L \rightarrow L$.

If $l \in \Omega^{2 n-1}(E) \mathrm{d} K \subseteq L$ has degree $2 n$, then $v(l)$ is a sum of elementary elements of $\mathrm{T} L$ with total degree at least $2 n$.

Let $M \subseteq E$ be a bounded $V$-submodule. There is a bounded subset $M^{\prime} \subseteq E$ such that if $e_{0} \mathrm{~d} e_{1} \ldots \mathrm{d} e_{2 n} \in \Omega^{2 n} M \cap L$, then $v\left(e_{0} \mathrm{~d} e_{1} \ldots \mathrm{d} e_{2 n}\right)$ is a sum of elementary elements of $\mathrm{T} L$ with entries in $M^{\prime}$.

Proof. As shown in 26 or in 25], the left action $\triangleright$ is by left multipliers, that is, $e \triangleright(\xi \odot \tau)=(e \triangleright \xi) \odot \tau$ for all $e \in E, \xi, \tau \in \mathrm{T} L$. And $k \triangleright \xi=k \odot \xi$ for all $k \in K$. This implies that $v$ is a homomorphism.

A short computation shows that each summand in the formula for $\omega_{\triangleright}\left(e_{1}, e_{2}\right)$ increases the total degree defined above by at least 2 ; this is already shown in the proof of 26 Lemma 5.102]. By induction on $n$, it follows that $v$ maps $\Omega^{2 n} L$ into the subgroup spanned by elementary elements of $\mathrm{T} L$ with total degree at least $2 n$.

Given a bounded subset $M \subseteq E$, the proof of [26 Lemma 5.92] provides a bounded subset $M^{\prime} \subseteq E$ such that $v\left(e_{0} \mathrm{~d} e_{1} \ldots \mathrm{d} e_{2 n} \odot k\right)$ is a sum of elementary elements of $\mathrm{T} L$ with entries in $M^{\prime}$.

The homomorphism $v$ induces an $F$-algebra homomorphism $L \otimes F \rightarrow \mathrm{T} L \otimes F$. Recall that

$$
L_{(m)}:=K \oplus \bigoplus_{n=1}^{\infty} \pi^{-\lfloor n / m\rfloor} \Omega^{2 n-1}(E) \mathrm{d} K
$$

for $m \in \mathbb{N}^{*}$. These are $V$-subalgebras of $L \otimes F$ that satisfy $L_{(n)} \subseteq L_{(m)}$ if $n \geq m$. Each $L_{(m)}$ is equipped with the bornology cofinally generated by the submodules in 5.1 .13 .

Let $(\mathrm{T} L)_{(m)} \subseteq \mathrm{T} L \otimes F$ be the subgroup generated by $\pi^{-\lfloor d / m\rfloor} \xi$ for elementary elements $\xi$ of total degree $d$. These are $V$-subalgebras of $\mathrm{T} L \otimes F$ that satisfy $(\mathrm{T} L)_{(n)} \subseteq(\mathrm{T} L)_{(m)}$ if $n \geq m$. If $M \subseteq E$ is a bounded $V$-submodule, then let $D_{m}^{\top}(M) \subseteq(\mathrm{T} L)_{(m)}$ be the subgroup generated by $\pi^{-\lfloor d / m\rfloor} \xi$ for elementary elements $\xi$ of total degree $d$. We give $(T L)_{(m)}$ the bornology that is cofinally generated by these $V$-submodules. This bornology is the analogue of the bornology in Corollary 4.4.14 
It is torsion-free and makes the multiplication in $(\mathrm{T} L)_{(m)}$ and the inclusion maps $(\mathrm{T} L)_{(n)} \hookrightarrow(\mathrm{T} L)_{(m)}$ for $n \geq m$ bounded. So we have turned $\left((\mathrm{T} L)_{(m)}\right)_{m \in \mathbb{N}^{*}}$ into a projective system of torsion-free bornological algebras.

The second paragraph in Lemma 5.2.2 says that the extension $L \otimes F \rightarrow \mathrm{T} L \otimes F$ of $v$ maps $L_{(m)}$ to $(\mathrm{T} L)_{(m)}$ for each $m \in \mathbb{N}^{*}$. And the third paragraph says that this homomorphism is bounded. Thus $v$ is a homomorphism of projective systems of bornological algebras. By Corollary 4.4.14 $\mathcal{L}$ is isomorphic to the projective system of the completions $\overline{L_{(m)}}$ for $m \in \mathbb{N}^{*}$, with the bornologies described above.

LEMma 5.2.3. The embedding $\mathrm{T} L \hookrightarrow \mathcal{T} L$ extends to an isomorphism of projective systems from the projective system of completions $\overline{(\mathrm{T} L)_{(m)}}$ for $m \in \mathbb{N}^{*}$ to $\mathcal{T} \mathcal{L}$.

Proof. For a bounded $V$-submodule $M \subseteq E$, let $M_{K}:=M \cap K$ and let $\bar{\Omega}_{\mathcal{L}}^{0}(M):=M_{K}$ and $\bar{\Omega}_{\mathcal{L}}^{2 k}(M):=\bar{\Omega}^{2 k-1}(M) \otimes M_{K}$ for $k>1$. A proof like that for Proposition 4.4.15 shows that the completion of $(\mathrm{T} L)_{(m)}$ is the union of the products

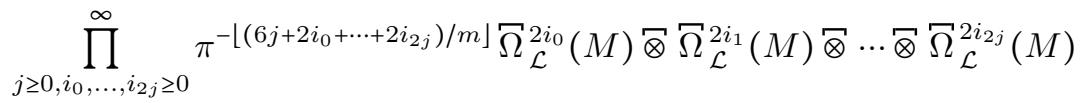

$$
\begin{aligned}
& \times \prod_{j \geq 0, i_{1}, \ldots, i_{2 j} \geq 0}^{\infty} \pi^{-\left\lfloor\left(6 j-4+2 i_{0}+\cdots+2 i_{2 j}\right) / m\right\rfloor} \widetilde{\Omega}_{\mathcal{L}}^{2 i_{1}}(M) \nabla \bar{\Omega}_{\mathcal{L}}^{2 i_{2}}(M) \otimes \cdots \nabla \bar{\Omega}_{\mathcal{L}}^{2 i_{2 j}}(M)
\end{aligned}
$$

taken over all bounded $V$-submodules $M \subseteq E$; elementary tensors in a factor of the first product correspond to differential forms $l_{0} \mathrm{D} l_{1} \ldots \mathrm{D} l_{2 j}$ with $l_{0}, \ldots, l_{2 j} \in \mathcal{L}$ and $\operatorname{deg}\left(l_{j}\right)=2 i_{j}$, whereas those for the second product correspond to differential forms $\mathrm{D} l_{1} \ldots \mathrm{D} l_{2 j}$. The exponent of $\pi$ is the total degree defined above.

Proposition 4.4.15 describes $\mathcal{T} E$. The pro-subalgebra $\mathcal{L}$ is described similarly, by also asking for the last entry of all differential forms to belong to $K$. Then a second application of Proposition 4.4.15 describes $\mathcal{T} \mathcal{L}$. The result is very similar to the projective system above. The only difference is that the exponent of $\pi$ in the bornology is replaced by $h:=\lfloor j / k\rfloor+\sum_{l=0}^{2 j}\left\lfloor i_{l} / m\right\rfloor$ for each factor in (5.2.4), for some parameters $k, m \in \mathbb{N}^{*}$. Here we may take $k=m$ because this gives a cofinal subset. So it remains to prove linear estimates between these two notions of "degree". In one direction, this is the trivial estimate

$$
\left\lfloor\frac{j}{m}\right\rfloor+\sum_{l=0}^{2 j}\left\lfloor\frac{i_{l}}{m}\right\rfloor \leq\left\lfloor\frac{j}{m}+\sum_{l=0}^{2 j} \frac{i_{l}}{m}\right\rfloor \leq\left\lfloor\frac{1}{m}\left(6 j+\sum_{l=0}^{2 j} 2 i_{l}\right)\right\rfloor
$$

for $j \geq 0$ and a similar estimate with $6 j-4=4(j-1)+2 j$ instead of $6 j$ for $j \geq 1$. In the other direction, we distinguish two cases. Let $i:=\sum i_{l}$. If $i<4 j \cdot m$, then $6 j+2 i<j \cdot(6+8 m)$ and we estimate

$$
\left\lfloor\frac{j}{m}\right\rfloor+\sum_{l=0}^{2 j}\left\lfloor\frac{i_{l}}{m}\right\rfloor \geq\left\lfloor\frac{j}{m}\right\rfloor \geq\left\lfloor\frac{6 j+2 i}{(6+8 m) \cdot m}\right\rfloor .
$$

The other case is $i \geq 4 j \cdot m$. Each floor operation changes a number by at most 1 , and $6 j+2 i \leq \frac{3}{2 m} i+2 i \leq 4 i$. So

$$
\left\lfloor\frac{j}{m}\right\rfloor+\sum_{l=0}^{2 j}\left\lfloor\frac{i_{l}}{m}\right\rfloor \geq \frac{i}{m}-2 j \geq \frac{i}{2 m} \geq\left\lfloor\frac{6 j+2 i}{8 m}\right\rfloor .
$$

As a result, $v$ defines a pro-algebra homomorphism $\mathcal{L} \rightarrow \mathcal{T} \mathcal{L}$. Then $\mathcal{L}$ is analytically quasi-free. This ends the proof of the excision theorem. 


\section{Stability with respect to algebras of matrices}

A matricial pair $(X, Y)$ consists of two torsion-free bornological modules $X$ and $Y$ and a surjective linear map $\langle\cdot, \cdot\rangle: Y \otimes X \rightarrow V$. Any such map is bounded. A homomorphism from $(X, Y)$ to another matricial pair $(W, Z)$ is a pair $f=\left(f_{1}, f_{2}\right)$ of bounded linear homomorphisms $f_{1}: X \rightarrow W, f_{2}: Y \rightarrow Z$ such that $\left\langle f_{2}(y), f_{1}(x)\right\rangle=$ $\langle y, x\rangle$ for all $x \in X$ and $y \in Y$. An elementary homotopy is a pair $H=\left(H_{1}, H_{2}\right)$ of bounded linear maps, where $H_{1}: X \rightarrow W[t]$ and $H_{2}: Y \rightarrow Z$ or $H_{1}: X \rightarrow W$ and $H_{2}: Y \rightarrow Z[t]$, such that the following diagram commutes:

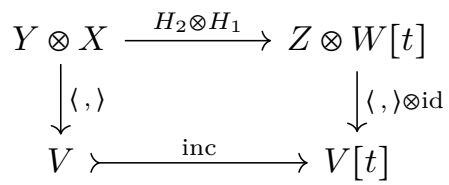

Let $(X, Y)$ be a matricial pair. Let $\mathcal{M}=\mathcal{M}(X, Y)$ be $X \otimes Y$ with the product

$$
\left(x_{1} \otimes y_{1}\right)\left(x_{2} \otimes y_{2}\right)=\left\langle y_{1}, x_{2}\right\rangle x_{1} \otimes y_{2} .
$$

This product is associative and bounded, and it even makes $\mathcal{M}$ a semi-dagger algebra. The bornological algebra $\mathcal{M}$ is also torsion-free by 27, Proposition 4.12]. Thus the completion $\overline{\mathcal{M}}$ is a dagger algebra and $\overline{\mathcal{M}}=\mathcal{M}^{\dagger}$.

Homomorphisms and homotopies of matricial pairs induce homomorphisms and homotopies of the corresponding algebras. Any pair $(\xi, \eta) \in X \times Y$ with $\langle\eta, \xi\rangle=1$ yields a bounded algebra homomorphism

$$
\iota=\iota_{\xi, \eta}: V \rightarrow \mathcal{M}, \quad \iota(1)=\xi \otimes \eta .
$$

We shall also write $\iota$ for the composite of the map above with the completion map $\mathcal{M} \rightarrow \overline{\mathcal{M}}=\mathcal{M}^{\dagger}$. If $R$ is a torsion-free bornological algebra, then $R \bar{\otimes} \mathcal{M}^{\dagger}$ is torsion-free by [27, Theorem 4.6 and Propositions 14.11 and 14.12]. Define

$$
\iota_{R}:=\operatorname{id}_{R} \otimes \iota: R \rightarrow R \nabla \mathcal{M}^{\dagger} .
$$

Proposition 6.2. Let $R$ be a complete, torsion-free bornological algebra. Then the map $\iota_{R}$ induces a chain homotopy equivalence $\mathbb{H} \mathbb{A}(R) \simeq \mathbb{H} \mathbb{A}\left(R \otimes \mathcal{M}^{\dagger}\right)$ and an isomorphism $\mathrm{HA}_{*}(R) \cong \mathrm{HA}_{*}\left(R \otimes \mathcal{M}^{\dagger}\right)$.

Proof. Corollary 4.5.6 yields a natural pro-algebra homomorphism $\mathcal{T}(R \bar{\otimes}$ $\left.\mathcal{M}^{\dagger}\right) \rightarrow \mathcal{T}(R) \Phi \mathcal{M}^{\dagger}$ covering the identity of $R \bar{\otimes} \mathcal{M}^{\dagger}$. And any elementary homotopy between matricial pairs $(X, Y)$ and $(W, Z)$ yields an elementary dagger homotopy $\mathcal{M}(X, Y)^{\dagger} \rightarrow \mathcal{M}(Z, W)^{\dagger} \otimes V[t]^{\dagger}$. The $X$-complex is invariant under dagger homotopies by Proposition 4.6.1. Taking all this into account, the argument of the proof of $[26$ Theorem 5.65] now applies verbatim and proves the proposition.

Let $\Lambda$ be a set. We now describe increasingly complicated algebras of matrices indexed by the set $\Lambda$.

EXAmple 6.3 . Let $\Lambda$ be a set and let $V^{(\Lambda)}$ be the $V$-module of finitely supported functions $\Lambda \rightarrow V$. This is the free module with basis $\left\{\chi_{\lambda}: \lambda \in \Lambda\right\}$ formed by the characteristic functions of the singletons. The algebra $\mathcal{M}\left(V^{(\Lambda)}, V^{(\Lambda)}\right)$ associated to the bilinear form $\left\langle\chi_{\lambda}, \chi_{\mu}\right\rangle=\delta_{\lambda, \mu}$ is just the algebra $M_{\Lambda}$ of finitely supported matrices indexed by $\Lambda \times \Lambda$, equipped with the fine bornology. The latter algebra is already a dagger algebra. Proposition 6.2 implies $\mathbb{H} \mathbb{A}(R) \cong \mathbb{H} \mathbb{A}\left(M_{\Lambda} \otimes R\right)$ for all $R$. 
Example 6.4. Define $V^{(\Lambda)}$ as in Example 6.3. Its $\pi$-adic completion is the Banach module $c_{0}(\Lambda):=c_{0}(\Lambda, V)$ with the supremum norm. The bilinear form in Example 6.3 extends to $c_{0}(\Lambda)$. The $\pi$-adic completion of $\mathcal{M}\left(c_{0}(\Lambda), c_{0}(\Lambda)\right)$ is isomorphic to the Banach $V$-algebra $M_{\Lambda}^{0} \cong c_{0}(\Lambda \times \Lambda)$ of matrices indexed by $\Lambda \times \Lambda$ with entries in $V$ that go to zero at infinity. The Banach $V$-modules above become bornological by declaring all subsets to be bounded. Then the completions and tensor products as Banach $V$-modules and as bornological $V$-modules are the same. Therefore, Proposition 6.2 implies $\mathbb{H} \mathbb{A}(R) \cong \mathbb{H} \mathbb{A}\left(M_{\Lambda}^{0} \otimes R\right)$ for all $R$.

EXAMPle 6.5. Let $\ell: \Lambda \rightarrow \mathbb{N}$ be a proper function, that is, for each $n \in \mathbb{N}$ the set of $x \in \Lambda$ with $\ell(x) \leq n$ is finite. Define $V^{(\Lambda)}$ as in Example 6.3 and give it the bornology that is cofinally generated by the $V$-submodules

$$
S_{m}:=\sum_{\lambda \in \Lambda} \pi^{\lfloor\ell(\lambda) / m\rfloor} \chi_{\lambda}
$$

for $m \in \mathbb{N}^{*}$. The bilinear form in Example 6.3 remains bounded for this bornology on $V^{(\Lambda)}$. So $\mathcal{M}\left(V^{(\Lambda)}, V^{(\Lambda)}\right)$ with the tensor product bornology from the above bornology is a bornological algebra as well. It is torsion-free and semi-dagger. So its dagger completion is the same as its completion. We denote it by $M_{\Lambda}^{\ell}$. It is isomorphic to the algebra of infinite matrices $\left(c_{x, y}\right)_{x, y \in \Lambda}$ for which there is $m \in \mathbb{N}^{*}$ such that $c_{x, y} \in \pi^{\lfloor(\ell(x)+\ell(y)) / m\rfloor}$ for all $x, y \in \Lambda$; this is the same as asking for $\lim \left|c_{x, y} \pi^{-\lfloor(\ell(x)+\ell(y)) / m\rfloor}\right|=0$ because $\ell$ is proper. It makes no difference to replace the exponent of $\pi$ by $\lfloor\ell(x) / m\rfloor+\lfloor\ell(y) / m\rfloor$ or $\lfloor\max \{\ell(x), \ell(y)\} / m\rfloor$ because we may vary $m$. Proposition 6.2 implies $\mathbb{H} \mathbb{A}(R) \cong \mathbb{H} \mathbb{A}\left(M_{\Lambda}^{\ell} \otimes R\right)$ for all $R$.

The following completed matrix algebras will be needed in Section 8

EXAMPLE 6.6. Let $\Lambda$ be a set with a filtration by a directed set $I$. That is, there are subsets $\Lambda_{S} \subseteq \Lambda$ for $S \in I$ with $\Lambda_{S} \subseteq \Lambda_{T}$ for $S \leq T$ and $\Lambda=\cup_{S \in I} \Lambda_{S}$. Let $\ell: \Lambda \rightarrow \mathbb{N}$ be a function whose restriction to $\Lambda_{S}$ is proper for each $S \in I$. For $S \in \Lambda$, form the matrix algebra $M_{\Lambda_{S}}^{\ell}$ as in Example 6.5 These algebras for $S \in I$ form an inductive system. Let $\lim _{\longrightarrow} M_{\Lambda_{S}}^{\ell}$ be its bornological inductive limit. This bornological algebra is also associated to a matricial pair, namely, the pair based on $\underset{\longrightarrow}{\longrightarrow} V^{\left(\Lambda_{S}\right)}$, where each $V^{\left(\Lambda_{S}\right)}$ carries the bornology described in Example 6.5 Thus Proposition 6.2 implies $\mathbb{H} \mathbb{A}(R) \cong \mathbb{H} \mathbb{A}\left(\lim _{\longrightarrow} M_{\Lambda_{S}}^{\ell} \otimes \vec{\otimes} R\right)$ for all $R$.

\section{Morita functoriality}

In this section, we show that analytic cyclic homology is functorial for certain bimodules. Let $A$ and $B$ be unital, torsion-free, complete bornological $V$-algebras and let $P$ be an $A$-B-bimodule. Assume $P$ to be finitely generated and projective as a right $B$-module. Then there are $n \in \mathbb{N}$ and an idempotent matrix $e \in \mathbb{M}_{n}(B)$ such that $P \cong e B^{n}$. The left action of $A$ on $P$ induces a $V$-algebra homomorphism

$$
\iota_{A}: A \rightarrow \operatorname{End}_{B}(P) \cong e \mathbb{M}_{n}(B) e \subseteq \mathbb{M}_{n}(B) .
$$

Proposition 6.2 describes a chain homotopy equivalence $\mathbb{H} \mathbb{A}(B) \cong \mathbb{H} \mathbb{A}\left(\mathbb{M}_{n}(B)\right)$ for any $n \in \mathbb{N}_{\geq 1}$. Composing this with the map induced by $\iota_{A}$ gives a chain map

$$
\mathbb{H} \mathbb{A}(P): \mathbb{H} \mathbb{A}(A) \rightarrow \mathbb{H} \mathbb{A}(B) .
$$

This induces maps $\mathrm{HA}_{*}(P): \mathrm{HA}_{*}(A) \rightarrow \mathrm{HA}_{*}(B)$ for $*=0,1$. 
LEMma 7.1. The homotopy class of $\mathbb{H} \mathbb{A}(P)$ only depends on the isomorphism class of $P$. That is, if $P \cong e \cdot B^{n} \cong f \cdot B^{m}$ for $n, m \in \mathbb{N}$ and idempotent $e \in \mathbb{M}_{n}(B)$, $f \in \mathbb{M}_{m}(B)$, then the resulting chain maps $\mathbb{H} \mathbb{A}(A) \rightarrow \mathbb{H} \mathbb{A}(B)$ are chain homotopic. If $A=B=P$, then $\mathbb{H} \mathbb{A}(P)$ is homotopic to the identity chain map.

Proof. The chain homotopy equivalence $\mathbb{H} \mathbb{A}(B) \cong \mathbb{H} \mathbb{A}\left(\mathbb{M}_{n}(B)\right)$ in Proposition 6.2 is induced by the corner embedding $\iota_{n}: B \rightarrow \mathbb{M}_{n}(B), b \mapsto b \cdot E_{11}$. If $k \geq n$, then the inclusion $j_{k n}: \mathbb{M}_{n}(B) \rightarrow \mathbb{M}_{k}(B)$ that appends zeros on the right and at the bottom satisfies $j_{k n} \circ \iota_{n}=\iota_{k}$. Hence there is a commuting diagram of chain homotopy equivalences

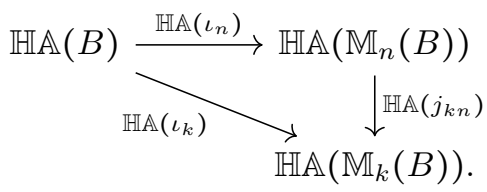

Therefore, the maps $\mathbb{H} \mathbb{A}(A) \rightarrow \mathbb{H} \mathbb{A}(B)$ remain unchanged when we replace $e$ and $f$ by $j_{k n}(e) \in \mathbb{M}_{k}(B)$ and $j_{k m}(f) \in \mathbb{M}_{k}(B)$ for $k \geq n, m$. This allows us to reduce to the case $n=m$. And then we may still choose $k=2 n=2 m$ to create extra room.

Since $f B^{m} \cong e B^{m}$, the idempotent matrices $e$ and $f$ are Murray-von-Neumann equivalent. That is, there are matrices $v, w \in \mathbb{M}_{m}(B)$ with

$$
e=v w, \quad f=w v, \quad v w v=v, \quad w v w=w .
$$

Let $\iota_{A}^{e}, \iota_{A}^{f}: A \rightrightarrows \mathbb{M}_{m}(B)$ be the two homomorphisms defined above using the idempotent elements $e$ and $f$, respectively. By construction, $\iota_{A}^{e}(a)=v \iota_{A}^{f}(a) w$ and $\iota_{A}^{f}(a)=w \iota_{A}^{e}(a) v$ for all $a \in A$. It is well known that $j_{2 m, m}(e)$ and $j_{2 m, m}(f)$ are homotopic. We recall the elementary proof. Let

$$
v_{t}:=t v+(1-t), \quad w_{t}:=t w+(1-t)
$$

in $\mathbb{M}_{m}(B[t])$ and let

$$
u_{t}:=\left(\begin{array}{cc}
v_{t} & v_{t} w_{t}-1 \\
1-w_{t} v_{t} & 2 w_{t}-w_{t} v_{t} w_{t}
\end{array}\right), \quad u_{t}^{-1}:=\left(\begin{array}{cc}
2 w_{t}-w_{t} v_{t} w_{t} & 1-w_{t} v_{t} \\
v_{t} w_{t}-1 & v_{t}
\end{array}\right) .
$$

Easy computations show that the latter two elements of $\mathbb{M}_{2 m}(B[t])$ satisfy $u_{0}=1$ and $u_{t} u_{t}^{-1}=1=u_{t}^{-1} u_{t}$. And

$$
u_{1}\left(\begin{array}{cc}
\iota_{A}^{f}(a) & 0 \\
0 & 0
\end{array}\right) u_{1}^{-1}=\left(\begin{array}{cc}
\iota_{A}^{e}(a) & 0 \\
0 & 0
\end{array}\right)
$$

for all $a \in A$. Therefore, conjugation by $u_{t}$ defines a polynomial homotopy between the homomorphisms $\iota_{A}^{e}$ and $\iota_{A}^{f}$. Since $\mathbb{H} \mathbb{A}$ is homotopy invariant by Theorem 4.6.2 it follows that the chain maps $\mathbb{H} \mathbb{A}(A) \rightarrow \mathbb{H} \mathbb{A}\left(\mathbb{M}_{2 m}(B)\right)$ induced by $\iota_{A}^{e}$ and $\iota_{A}^{f}$ are chain homotopic.

To prove the last claim about $\mathbb{H} \mathbb{A}(A)$ for the identity bimodule $A$, use $m=1$ and $e=1$. Then $\iota_{A}: A \rightarrow \mathbb{M}_{m}(A)$ is the identity map.

Lemma 7.2. Let $A, B, C$ be unital, torsion-free, complete bornological $V$-algebras and let $P$ be an $A$-B-bimodule and $Q$ a $B$-C-bimodule. Assume $P$ and $Q$ to be finitely generated and projective as right modules. Then $P \otimes_{B} Q$ is finitely generated and projective as a right module, and $\mathbb{H} \mathbb{A}\left(P \otimes_{B} Q\right)$ is chain homotopic to $\mathbb{H} \mathbb{A}(P) \circ \mathbb{H} \mathbb{A}(Q)$. 
Proof. By assumption, there are $m, n \in \mathbb{N}$ and idempotent matrices $e \in \mathbb{M}_{m}(B)$ and $f \in \mathbb{M}_{n}(C)$ with $P \cong e B^{m}$ and $Q \cong f C^{n}$. Then

$P \otimes_{B} Q \cong\left(e \cdot B^{m}\right) \otimes_{B}\left(f \cdot C^{n}\right) \cong\left(e \otimes_{B} 1\right) \cdot\left(B^{m} \otimes_{B}\left(f \cdot C^{n}\right)\right)=\left(e \otimes_{B} 1\right) \cdot\left(f \cdot C^{n}\right)^{m}$.

More precisely, this identifies $P \otimes_{B} Q$ with the image of $\mathbb{M}_{m}\left(\iota_{B}\right)(e) \in \mathbb{M}_{m \cdot n}(C)$, where $\iota_{B}: B \rightarrow \mathbb{M}_{n}(C)$ is the homomorphism associated to $f$ and $\mathbb{M}_{m}\left(\iota_{B}\right): \mathbb{M}_{m}(B) \rightarrow$ $\mathbb{M}_{m \cdot n}(C)$ is the induced homomorphism on matrices. Inspection shows that the homomorphism $A \rightarrow \mathbb{M}_{m \cdot n}(C)$ defined by realising $P \otimes_{B} Q$ in this way is equal to the composite homomorphism $\mathbb{M}_{m}\left(\iota_{B}\right) \circ \iota_{A}: A \rightarrow \mathbb{M}_{m}(B) \rightarrow \mathbb{M}_{m \cdot n}(C)$. This implies the claim because the chain homotopy equivalences $\mathbb{H} \mathbb{A}(B) \cong \mathbb{H} \mathbb{A}\left(\mathbb{M}_{m}(B)\right)$ are natural.

Theorem 7.3. Let $A$ and $B$ be unital, torsion-free, complete bornological $V$-algebras. A Morita equivalence between them induces a chain homotopy equivalence $\mathbb{H} \mathbb{A}(A) \simeq \mathbb{H} \mathbb{A}(B)$ and isomorphisms $\mathrm{HA}_{*}(A) \cong \mathrm{HA}_{*}(B)$ for $*=0,1$.

Proof. The Morita equivalence is given by bimodules $P$ and $Q$ over $A, B$ and $B, A$ with $P \otimes_{B} Q \cong A$ and $Q \otimes_{A} P \cong B$. It is well known that the equivalence bimodules $P$ and $Q$ are finitely generated and projective as right modules. Hence they induce well defined chain maps $\mathbb{H} \mathbb{A}(A) \leftrightarrow \mathbb{H} \mathbb{A}(B)$ by the construction above. These are inverse up to chain homotopy by Lemma 7.2 This homotopy equivalence implies isomorphisms $\mathrm{HA}_{*}(A) \cong \mathrm{HA}_{*}(B)$ for $*=0,1$ on analytic cyclic homology.

When dealing with non-unital algebras, Morita theory gets more difficult. In particular, we know less about the bimodules involved in a Morita equivalence. The issue is to impose the right assumptions on an $A, B$-bimodule so that there are a matricial pair as in Section 6 , an idempotent double centraliser $e$ of $B \bar{\otimes} \overrightarrow{\mathcal{M}}$, and an algebra homomorphism $A \rightarrow e(B \otimes \overline{\mathcal{M}}) e$. We do not discuss sufficient conditions on bimodules that allow to associate such data to them.

\section{Leavitt path algebras}

Our next goal is to compute the analytic cyclic homology for tensor products with Leavitt and Cohn path algebras of directed graphs and their dagger completions. A directed graph $E$ consists of a set $E^{0}$ of vertices and a set $E^{1}$ of edges together with source and range maps $s, r: E^{1} \rightarrow E^{0}$. A vertex $v \in E^{0}$ is regular if $0<\left|s^{-1}(\{v\})\right|<\infty$. Let $\operatorname{reg}(E) \subseteq E^{0}$ be the subset of regular vertices. Define

$$
N_{E}: E^{0} \times \operatorname{reg}(E) \rightarrow \mathbb{Z}, \quad(v, w) \mapsto \delta_{v, w}-\left|s^{-1}(\{w\}) \cap r^{-1}(\{v\})\right| .
$$

Let $L(E)$ and $C(E)$ be the Leavitt and Cohn path algebras over $V$, as defined in [1. Definitions 1.2.3 and 1.2.5]. We consider them as bornological algebras with the fine bornology. The following theorem follows easily from the results in $1 \mathbf{1 3}$ and the formal properties of analytic cyclic homology:

Theorem 8.1. Assume char $F=0$. Let $R$ be a complete bornological algebra. Let $E$ be a graph with countably many vertices. Then

$$
\begin{array}{ll}
\mathbb{H} \mathbb{A}(R \otimes C(E)) \simeq \mathbb{H} \mathbb{A}\left(R \otimes V^{\left(E^{0}\right)}\right), \quad & \mathbb{H} \mathbb{A}(C(E)) \simeq F^{\left(E^{0}\right)}, \\
& \mathbb{H} \mathbb{A}(L(E)) \simeq \operatorname{coker}\left(N_{E}\right) \oplus \operatorname{ker}\left(N_{E}\right)[1],
\end{array}
$$


If $E^{0}$ is finite, then

$$
\begin{aligned}
& \mathbb{H} \mathbb{A}(R \otimes C(E)) \simeq \bigoplus_{v \in E^{0}} \mathbb{H} \mathbb{A}(R), \\
& \mathbb{H} \mathbb{A}(R \otimes L(E)) \simeq\left(\operatorname{coker}\left(N_{E}\right) \oplus \operatorname{ker}\left(N_{E}\right)[1]\right) \otimes \mathbb{H} \mathbb{A}(R) .
\end{aligned}
$$

Proof. We define a functor $H$ from the category of $V$-algebras to the triangulated category of pro-supercomplexes by giving $A$ the fine bornology and taking $\mathbb{H} \mathbb{A}(R \otimes A)$. The functor $H$ is homotopy invariant for polynomial (and even dagger) homotopies by Theorem 4.6.2 stable for algebras of finite matrices over any set $\Lambda$ by Proposition 6.2 applied to Example 6.3, and exact on semi-split extensions by Theorem 5.1 Theorem 5.1 also implies that $\mathbb{H} \mathbb{A}$ is finitely additive. It is not countably additive in general, but Corollary 4.3 .9 shows that it is countably additive on the ground ring $V$. Now $\mathbf{1 3}$, Theorem 4.2] proves a homotopy equivalence

$$
\mathbb{H} \mathbb{A}(R \otimes C(E)) \simeq \mathbb{H} \mathbb{A}\left(R \otimes V^{\left(E^{0}\right)}\right) .
$$

If $E^{0}$ is finite, then this is homotopy equivalent to $\mathbb{H} \mathbb{A}(R) \otimes V^{\left(E^{0}\right)}=\oplus_{v \in E^{0}} \mathbb{H} \mathbb{A}(R)$ by finite additivity. And if $R=V$, then Corollary 4.3.9 identifies $\mathbb{H} \mathbb{A}\left(V^{\left(E^{0}\right)}\right) \simeq F^{\left(E^{0}\right)}$.

13 Proposition 5.2] yields a distinguished triangle of pro-supercomplexes

$$
\mathbb{H} \mathbb{A}\left(R \otimes V^{(\mathrm{reg}(E))}\right) \stackrel{f}{\rightarrow} \mathbb{H} \mathbb{A}\left(R \otimes V^{\left(E^{0}\right)}\right) \rightarrow \mathbb{H} \mathbb{A}(R \otimes L(E)) \rightarrow \mathbb{H} \mathbb{A}\left(R \otimes V^{(\mathrm{reg}(E))}\right)
$$

and partly describes the map $f$. If $R=V$ and $E^{0}$ is countable, then Corollary 4.3.9 identifies $\mathbb{H} \mathbb{A}\left(V^{\left(E^{0}\right)}\right) \simeq F^{\left(E^{0}\right)}$ and $\mathbb{H} \mathbb{A}\left(V^{\mathrm{reg}(E)}\right) \simeq F^{\mathrm{reg}(E)}$, and the information about the map $f$ in [13. Proposition 5.2] shows that it multiplies vectors with the matrix $N_{E}$. If $E^{0}$ is finite, then $\mathbb{H} \mathbb{A}$ is $E^{0}$-additive and [13. Theorem 5.4] gives a distinguished triangle

$$
\mathbb{H} \mathbb{A}(R) \otimes F^{\mathrm{reg}(E)} \stackrel{\operatorname{id} \otimes N_{E}}{\longrightarrow} \mathbb{H} \mathbb{A}(R) \otimes F^{E^{0}} \rightarrow \mathbb{H} \mathbb{A}(R \otimes L(E)) \rightarrow \cdots .
$$

Since $\operatorname{char}(F)=0$, there are invertible matrices $x, y$ with entries in $F$ such that $x N_{E} y$ is a diagonal matrix with only zeros and ones in the diagonal. We may replace the map $N_{E}$ or id $\otimes N_{E}$ above by id $\otimes\left(x N_{E} y\right)$. Then the formulas for $\mathbb{H} \mathbb{A}(L(E))$ in general and for $\mathbb{H} \mathbb{A}(R \otimes L(E))$ for finite $E^{0}$ follow.

COROLlary 8.2. $\mathbb{H} \mathbb{A}\left(R \otimes V\left[t, t^{-1}\right]\right)$ is chain homotopy equivalent to $\mathbb{H} \mathbb{A}(R) \oplus$ $\mathbb{H} \mathbb{A}(R)[1]$ and $\mathrm{HA}_{*}\left(R \otimes V\left[t, t^{-1}\right]\right) \cong \mathrm{HA}_{*}(R) \oplus \mathrm{HA}_{*}(R)[1]$.

Proof. Apply Theorem 8.1 to the graph consisting of one vertex and one loop.

The following theorem says that Theorem 8.1 remains true for the dagger completions $C(E)^{\dagger}$ and $L(E)^{\dagger}$ of $C(E)$ and $L(E)$ :

THEOREM 8.3. Let $R$ be a complete bornological algebra and let $E$ be a graph. Then

$$
\mathbb{H} \mathbb{A}(R \otimes C(E)) \simeq \mathbb{H} \mathbb{A}\left(R \otimes C(E)^{\dagger}\right), \quad \mathbb{H} \mathbb{A}(R \otimes L(E)) \simeq \mathbb{H} \mathbb{A}\left(R \otimes L(E)^{\dagger}\right) .
$$

So the formulas in Theorem 8.1 also compute $\mathbb{H} \mathbb{A}\left(R \bar{\otimes} C(E)^{\dagger}\right)$ and $\mathbb{H} \mathbb{A}(R \bar{\otimes}$ $\left.L(E)^{\dagger}\right)$ - assuming $E^{0}$ to be countable or finite or $R=V$ for the different cases.

CoROLlary 8.4 (Fundamental Theorem). $\mathbb{H} \mathbb{A}\left(R \otimes V\left[t, t^{-1}\right]^{\dagger}\right)$ is chain homotopy equivalent to $\mathbb{H} \mathbb{A}(R) \oplus \mathbb{H} \mathbb{A}(R)[1]$ and $\mathrm{HA}_{*}\left(R \otimes V\left[t, t^{-1}\right]^{\dagger}\right) \cong \mathrm{HA}_{*}(R) \oplus \mathrm{HA}_{*}(R)[1]$.

Proof. Combine Theorem 8.3 and Corollary 8.2 . 
We are going to prove Theorem 8.3 by showing that the proofs in $\mathbf{1 3}$ continue to work when we suitably complete all algebras that occur there. We must be careful, however, because the dagger completion is not an exact functor. We first recall some basic facts that are used in $\mathbf{1 3}$. These will be used to describe the dagger completions $C(E)^{\dagger}$ and $L(E)^{\dagger}$.

By definition, $L(E)$ has the same generators as $C(E)$ and more relations. This provides a quotient map $p: C(E) \rightarrow L(E)$. Let $K(E) \subseteq C(E)$ be its kernel.

Lemma 8.5. There is a semi-split extension of $V$-algebras

$$
K(E) \nrightarrow C(E) \rightarrow L(E) .
$$

Proof. Let $\mathcal{P}$ be the set of finite paths in $E$. For $v \in \operatorname{reg}(E)$, choose $e_{v} \epsilon$ $s^{-1}(\{v\})$. Let

$$
\begin{aligned}
\mathcal{B} & :=\left\{\alpha \beta^{*}: \alpha, \beta \in \mathcal{P}, r(\alpha)=r(\beta)\right\}, \\
\mathcal{B}^{\prime} & :=\mathcal{B} \backslash\left\{\alpha e_{v} e_{v}^{*} \beta^{*}: v \in \operatorname{reg}(E), \alpha, \beta \in \mathcal{P}, r(\alpha)=r(\beta)=v\right\} .
\end{aligned}
$$

By [1. Propositions 1.5.6 and 1.5.11], $\mathcal{B}$ is a basis of $C(E)$ and $\mathcal{B}^{\prime}$ is a basis of $L(E)$. Let $\sigma: L(E) \rightarrow C(E)$ be the linear map that sends each element of $\mathcal{B}^{\prime}$ to itself. This is a section for the quotient map $p: C(E) \rightarrow L(E)$.

Next we describe $K(E)$ as in 1 . Proposition 1.5.11]. Let $v \in \operatorname{reg}(E)$. Define

$$
q_{v}:=v-\sum_{s(e)=v} e e^{*} .
$$

Let $\mathcal{P}_{v} \subseteq \mathcal{P}$ be the set of all paths with $r(\alpha)=v$. Let $V^{\left(\mathcal{P}_{v}\right)}$ be the free $V$-module on the set $\mathcal{P}_{v}$ and let $\mathcal{M}_{\mathcal{P}_{v}}$ be the algebra of finite matrices indexed by $\mathcal{P}_{v}$ as in Example 6.3 The map

$$
\bigoplus_{v \in \operatorname{reg}(E)} \mathcal{M}_{\mathcal{P}_{v}} \rightarrow K(E), \quad \alpha \otimes \beta \mapsto \alpha q_{v} \beta^{*},
$$

is a $V$-algebra isomorphism by [1. Proposition 1.5.11]. Each $\mathcal{M}_{\mathcal{P}_{v}}$ with the fine bornology is a dagger algebra because it is a union of finite-dimensional subalgebras. Thus $K(E)$ is a dagger algebra as well. In contrast, $C(E)$ and $L(E)$ with the fine bornology are not semi-dagger algebras. And the restriction to $K(E)$ of the linear growth bornology of $C(E)$ is not just the fine bornology: this is already visible in the special case where $C(E)$ is the Toeplitz algebra and $L(E)=V\left[t, t^{-1}\right]$.

We are going to describe the linear growth bornology on $C(E)$. Let $\mathcal{F}$ be the set of all finite subsets $S \subseteq E^{0} \cup E^{1}$ such that

$$
e \in S \cap E^{1} \text { and } s(e) \in \operatorname{reg}(E) \Rightarrow\{s(e)\} \cup s^{-1}(s(e)) \subseteq S .
$$

Let $S^{(\infty)}$ for $S \in \mathcal{F}$ be the set of all paths that consist only of edges in $S$. Let $|\alpha|$ be the length of a path $\alpha \in \mathcal{P}$. For $n \in \mathbb{N}$, let

$$
S_{n}:=\left\{\alpha \beta^{*}: \alpha, \beta \in S^{(\infty)},|\alpha|+|\beta| \leq n\right\} \subseteq \mathcal{B} .
$$

This is an increasing filtration on the basis $\mathcal{B}$ of $C(E)$.

Lemma 8.6. A subset of $C(E)$ has linear growth if and only if there are $S \in \mathcal{F}$ and $m \in \mathbb{N}^{*}$ such that it is contained in the $V$-linear span of $\cup_{n \in \mathbb{N}} \pi^{\lfloor n / m\rfloor} S_{n}$. 
Proof. It is easy to see that the $V$-linear span of $\bigcup_{n \in \mathbb{N}} \pi^{\lfloor n / m\rfloor} S_{n}$ in $C(E)$ has linear growth. Conversely, we claim that any subset of linear growth is contained in one of this form. Every finite subset of $E^{0} \cup E^{1}$ is contained in an element of $\mathcal{F}$. It follows that, for every finitely generated submodule $M \subseteq C(E)$, there are $S \in \mathcal{F}$ and $m \geq 1$ such that $M$ is contained in the $V$-submodule generated by $S_{m}$. Then $M^{j}$ is contained in the $V$-submodule generated by $S_{m j}$ for all $j \in \mathbb{N}^{*}$. Thus $M^{\diamond}$ is contained in the $V$-submodule generated by $\pi^{j-1} S_{m j}$ for all $j \in \mathbb{N}^{*}$. This is the $V$-linear span of $\bigcup_{n \in \mathbb{N}^{*}} \pi^{\lceil n / m\rceil-1} S_{n}$. Letting $m$ vary, we may replace $\lceil n / m\rceil-1$ by $\lfloor n / m\rfloor$.

Constructing linear growth bornologies commutes with taking quotients. So a subset of $L(E)$ has linear growth if and only if it is the image of a subset of linear growth in $C(E)$. Next we show that the section $\sigma: L(E) \rightarrow C(E)$ is bounded for the linear growth bornologies, and we describe the restriction to $K(E)$ of the linear growth bornology on $C(E)$ :

LEMMA 8.7. Give $V^{\left(\mathcal{P}_{v}\right)} \subseteq V^{(\mathcal{P})}$ the bornology where a subset is bounded if and only if it is contained in the linear span of $\left\{\pi^{\lfloor|\alpha| / m\rfloor} \alpha: \alpha \in S^{(\infty)}\right\}$ for some $S \in \mathcal{F}$ and some $m \in \mathbb{N}^{*}$. Equip the matrix algebra $\mathcal{M}_{\mathcal{P}_{v}}=V^{\left(\mathcal{P}_{v} \times \mathcal{P}_{v}\right)}$ with the resulting tensor product bornology and the multiplication defined by the obvious bilinear pairing as in Section 6, and give $\bigoplus_{v \in \operatorname{reg}(E)} \mathcal{M}_{\mathcal{P}_{v}}$ the direct sum bornology. There is a semi-split extension of bornological algebras

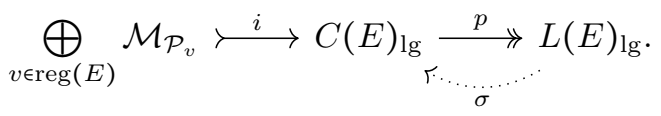

Proof. Let $S \in \mathcal{F}$. We claim that $\sigma \circ p$ maps the linear span of $S_{n}$ into itself. If $\alpha \beta^{*} \in \mathcal{B}^{\prime}$, then $\sigma \circ p\left(\alpha \beta^{*}\right)=\alpha \beta^{*}$. If $\alpha \beta^{*} \notin \mathcal{B}^{\prime}$, then $\alpha=\alpha_{0} e_{v}, \beta=\beta_{0} e_{v}$ for some $v \in \operatorname{reg}(E), \alpha_{0}, \beta_{0} \in \mathcal{P}_{v}$. And then

$$
p\left(\alpha \beta^{*}\right)=p\left(\alpha_{0} \beta_{0}^{*}\right)-\sum_{s(e)=v, e \neq e_{v}} p\left(\alpha_{0} e e^{*} \beta_{0}\right) .
$$

Since $\alpha_{0} \beta_{0}^{*}$ is shorter than $\alpha \beta^{*}$ and $\alpha_{0} e e^{*} \beta_{0} \in \mathcal{B}^{\prime}$ for $e \in E^{1}$ with $s(e)=v$ and $e \neq e_{v}$, an induction over $|\alpha|+|\beta|$ shows that $\sigma \circ p\left(\alpha \beta^{*}\right)$ is always a $V$-linear combination of shorter words; in addition, all edges in these words are again contained in $S$ because $S \in \mathcal{F}$. This proves the claim. Now Lemma 8.6 implies that $\sigma \circ p$ preserves linear growth of subsets. Equivalently, $\sigma$ is a bounded map $L(E)_{\lg } \rightarrow C(E)_{\lg }$. Then a subset of $K(E)$ has linear growth in $C(E)$ if and only if it is of the form (id $-\sigma \circ p)(M)$ for a $V$-submodule $M \subseteq C(E)$ that has linear growth. The projection id $-\sigma \circ p$ kills $\alpha \beta^{*} \in \mathcal{B}^{\prime}$. Thus we may disregard these generators when we describe the restriction to $K(E)$ of the linear growth bornology on $C(E)$. Instead of applying id $-\sigma \circ p$ to the remaining basis vectors $\alpha e_{v} e_{v}^{*} \beta^{*}$ for $r(\alpha)=r(\beta)=v \in \operatorname{reg}(E)$, we may also apply it to $\alpha e_{v} e_{v}^{*} \beta^{*}-\alpha \beta^{*}$ because $\alpha \beta^{*}$ is a shorter basis vector that involves the same edges. And

$$
\begin{aligned}
(\mathrm{id}-\sigma \circ p)\left(\alpha e_{v} e_{v}^{*} \beta^{*}-\alpha \beta^{*}\right) & =\alpha e_{v} e_{v}^{*} \beta^{*}-\alpha \beta^{*}+\sigma\left(\sum_{s(e)=v, e \neq e_{v}} p\left(\alpha e e^{*} \beta^{*}\right)\right) \\
& =-\alpha \beta^{*}+\sum_{s(e)=v} \alpha e e^{*} \beta^{*}=-\alpha q_{v} \beta^{*} .
\end{aligned}
$$

Now Lemma 8.6 implies that a subset of $K(E)$ has linear growth in $C(E)$ if and only if there are $S \in \mathcal{F}$ and $m \in \mathbb{N}^{*}$ so that it belongs to the $V$-linear span of $\pi^{\lfloor n / m\rfloor} \alpha q_{v} \beta^{*}$ 
with $v \in \operatorname{reg}(E), \alpha, \beta \in \mathcal{P}_{v} \cap S^{(\infty)}$, and $|\alpha|+|\beta|+2 \leq n$. Under the isomorphism $\oplus_{v \in \operatorname{reg}(E)} \mathcal{M}_{\mathcal{P}_{v}} \cong K(E)$, this becomes equal to the bornology on $\oplus_{v \in \operatorname{reg}(E)} \mathcal{M}_{\mathcal{P}_{v}}$ specified in the statement of the lemma.

The semi-split extension in Lemma 8.7 implies a similar semi-split extension involving the dagger completions $C(E)^{\dagger}, L(E)^{\dagger}$ and the completion of $\oplus_{v \in \operatorname{reg}(E)} \mathcal{M}_{\mathcal{P}_{v}}$ for the bornology specified in Lemma 8.7

Now Theorem 8.3 is proven by showing that all homomorphisms and quasihomomorphisms that are used in $\mathbf{1 3}$ remain bounded and all homotopies among them remain dagger homotopies when we give all algebras that occur the suitable "linear growth" bornology, defined using the lengths of paths to define linear growth. This is because all maps in $\mathbf{1 3}$ are described by explicit formulas in terms of paths, which change the length only by finite amounts. We have put linear growth in quotation marks because the correct bornologies on the ideals $K(E)$ and $\hat{K}(E)$ in 13 are restrictions of linear growth bornologies on larger algebras as in Lemma 8.7 These bornological algebras are special cases of Example 6.6, and so $\mathbb{H} \mathbb{A}$ is stable for such matrix algebras. The bornology on $K(E)$ in Lemma 8.7 actually deserves to be called a "linear growth bornology". But the relevant length function is specified by hand and not by the length of products as for the official linear growth bornology in Definition 2.1.16

\section{Filtered Noetherian rings and analytic quasi-freeness}

In Section 9.1 we develop a criterion for a quasi-free algebra to be analytically quasi-free. It uses a connection with a growth condition, called finite-degree. In Section 9.2 we show that the criterion from Section 9.1 applies to dagger completions of smooth, commutative $V$-algebras of relative dimension 1 . And we show that any smooth curve over $\mathbb{F}$ lifts to such a $V$-algebra.

9.1. Finite-degree connections. Recall that a complete bornological $V$-algebra $R$ is called quasi-free if all its square-zero extensions split. This is equivalent to the existence of a connection on $\bar{\Omega}^{1}(R)$, that is, a linear map $\nabla: \bar{\Omega}^{1}(R) \rightarrow \bar{\Omega}^{2}(R)$ satisfying

$$
\nabla(a \omega)=a \nabla(\omega) \text { and } \quad \nabla(\omega a)=\nabla(\omega) a+\omega \mathrm{d} a,
$$

for all $a \in R$ and $\omega \in \widetilde{\Omega}^{1}(R)$. And this is, in turn, equivalent to $\widetilde{\Omega}^{1}(R)$ being projective for extensions of complete bornological $R$-bimodules with a bounded $V$-linear section. The above claims go back to Cuntz and Quillen [16 for algebras without extra structure. They also hold for algebras in additive symmetric monoidal categories and hence for complete bornological $V$-algebras (see, for instance, $[\mathbf{2 6}]$ ). A related result is Proposition 4.4.6.

We are going to prove that a quasi-free algebra $R$ is analytically quasi-free if $\bar{\Omega}^{1}(R)$ has a connection whose growth is controlled in a certain way. This uses increasing filtrations. An (increasing) filtration on a $V$-module $M$ is an increasing sequence of $V$-submodules $\left(\mathcal{F}_{n} M\right)_{n \in \mathbb{N}}$ with $\cup \mathcal{F}_{n} M=M$. For a $V$-algebra $R$, we require, in addition, that $\mathcal{F}_{n} R \cdot \mathcal{F}_{m} R \subseteq \mathcal{F}_{n+m} R$ for all $n, m \in \mathbb{N}$. And for a module $M$ over a $V$-algebra $R$ with a fixed filtration $\left(\mathcal{F}_{n} R\right)_{n \in \mathbb{N}}$, we require, in addition, that $\mathcal{F}_{n} R \cdot \mathcal{F}_{m} M \subseteq \mathcal{F}_{n+m} M$ for all $n, m \in \mathbb{N}$. Then we speak of a filtered algebra and a filtered module, respectively. 
Definition 9.1.1. A map $f: M \rightarrow N$ between filtered $V$-modules has finite degree if there is $a \in \mathbb{N}$ - the degree - such that $f\left(\mathcal{F}_{n} M\right) \subseteq \mathcal{F}_{n+a}(N)$ for all $n \in \mathbb{N}$. Two filtrations $\left(\mathcal{F}_{n} M\right)_{n}$ and $\left(\mathcal{F}_{n}^{\prime} M\right)_{n}$ on a filtered $V$-module $M$ are called shift equivalent if there is $a \in \mathbb{N}$ such that $\mathcal{F}_{n} M \subseteq \mathcal{F}_{n+a}^{\prime} M$ and $\mathcal{F}_{n}^{\prime} M \subseteq \mathcal{F}_{n+a} M$ for all $n \in \mathbb{N}$.

EXAmple 9.1.2. Let $R$ be a torsion-free bornological $V$-algebra. Define $M^{(j)}$ for a complete bounded submodule $M \subseteq R$ and $j \geq 0$ as in (2.2.1). Put

$$
\mathcal{F}_{r}^{M} \widetilde{\Omega}^{j} R:=\sum_{i_{0}+\cdots+i_{j} \leq r} M^{\left(i_{0}\right)} \mathrm{d} M^{\left(i_{1}\right)} \ldots \mathrm{d} M^{\left(i_{j}\right)} \oplus \sum_{i_{1}+\cdots+i_{j} \leq r} \mathrm{~d} M^{\left(i_{1}\right)} \ldots \mathrm{d} M^{\left(i_{j}\right)}
$$

for $r \in \mathbb{N}$. This is an increasing filtration on the differential $j$-forms of the subalgebra $M^{(\infty)} \subseteq R$ generated by $M$.

The following lemma relates such filtrations to the linear growth bornology:

Lemma 9.1.4. Let $R$ be a torsion-free bornological algebra, $M \subseteq R$ a bounded $V$-submodule and $n \geq 0$. Then

$$
\sum_{i \geq 0} \pi^{i} \mathcal{F}_{i+n}^{M} \bar{\Omega}^{n} R \subseteq \bar{\Omega}^{n}\left(M^{\diamond}\right) \subseteq \sum_{i \geq 0} \pi^{i} \mathcal{F}_{i+n+1}^{M} \bar{\Omega}^{n} R .
$$

Proof. We compute

$$
\begin{aligned}
\bar{\Omega}^{n}\left(M^{\diamond}\right)= & M^{\diamond} \mathrm{d}\left(M^{\diamond}\right)^{n} \oplus \mathrm{d}\left(M^{\diamond}\right)^{n} \\
= & \sum_{i \geq 0} \pi^{i}\left(\sum_{i_{0}+\cdots+i_{n}=i} M^{\left(i_{0}+1\right)} \mathrm{d} M^{\left(i_{1}+1\right)} \ldots \mathrm{d} M^{\left(i_{n}+1\right)}\right. \\
& \left.\oplus \sum_{i_{1}+\cdots+i_{n}=i} \mathrm{~d} M^{\left(i_{1}+1\right)} \ldots \mathrm{d} M^{\left(i_{n}+1\right)}\right) .
\end{aligned}
$$

Lemma 9.1.5. Let $M \subseteq R$ be a bounded submodule, $r, b \geq 1$ and $s \geq 0$. Then

$$
\mathcal{F}_{r}^{M} \bar{\Omega}^{s} R \subseteq \mathcal{F}_{\lceil r / b\rceil+s}^{M^{(b)}} \bar{\Omega}^{s} R \subseteq \mathcal{F}_{r+b(s+1)}^{M} \bar{\Omega}^{s} R .
$$

Proof. Straightforward.

Lemma 9.1.6. Let $X$ and $Y$ be torsion-free bornological modules. Let $\left(f_{n}\right)$ be a sequence of bounded linear maps $X \rightarrow Y$. Assume that for each bounded submodule $M \subseteq X$ there is a bounded submodule $N \subseteq Y$ and a sequence of nonnegative integers $\left(a_{n}\right)$ with $\lim a_{n}=\infty$ and $f_{n}(M) \subseteq \pi^{a_{n}} N$ for all $n \in \mathbb{N}$. Then the series $s(x):=\sum_{n} f_{n}(x)$ converges in $\bar{Y}$ for every $x \in X$, and the assignment $x \mapsto s(x)$ is bounded and linear. So it extends to a bounded linear map $s: X \rightarrow Y$.

Proof. Straightforward.

Definition 9.1.7. Let $R$ be a torsion-free bornological $V$-algebra. A connection $\nabla: \widetilde{\Omega}^{1}(R) \rightarrow \widetilde{\Omega}^{2}(R)$ has finite degree on a bounded submodule $M \subseteq R$ if it has finite degree as a $V$-module map with respect to the filtrations on $\bar{\Omega}^{1}\left(M^{(\infty)}\right)$ and $\bar{\Omega}^{2}\left(M^{(\infty)}\right)$ from Example 9.1.2 A connection $\nabla$ has finite degree on $R$ if any bounded subset is contained in a bounded submodule of $R$ on which $\nabla$ has finite degree.

REMARK 9.1.8. Lemma 9.1.5 implies that if $\nabla$ has finite degree on $M$, then it also has finite degree on $M^{(b)}$ for all $b$. Then $\nabla$ is a finite degree connection on $M^{(\infty)}$ with the bornology that is cofinally generated by $M^{(n)}$ for $n \in \mathbb{N}$. 
The following theorem is an analytic version of the formal tubular neighbourhood theorem by Cuntz and Quillen in $\mathbf{1 6}$.

THEOREM 9.1.9. Let $R$ be a complete, torsion-free bornological algebra. If $\Omega^{1}(R)$ has a connection of finite degree, then $R^{\dagger}$ is analytically quasi-free.

Proof. We introduce some notation on Hochschild cochains. If $X$ is a complete, bornological $R$-bimodule and $\psi: R^{\bar{\otimes}_{n}} \rightarrow X$ is an $n$-cochain, write $\delta(\psi)$ for its Hochschild coboundary. If $\xi: R^{\otimes} m \rightarrow Y$ is another cochain, write $\psi \cup \xi: R^{\mathbb{\otimes} n+m} \rightarrow$ $X \Phi_{R} Y$ for the cup product. Let $\nabla: \bar{\Omega}^{1} R \rightarrow \widetilde{\Omega}^{2} R$ be a connection of finite degree, and let $M \subseteq R$ be a bounded submodule and $a \geq 0$ an integer such that $\nabla$ has degree $a$ on $M$. The connection $\nabla$ is equivalent to a 1-cochain $\varphi_{2}: R \rightarrow \bar{\Omega}^{2} R$ satisfying $\delta\left(\varphi_{2}\right)=\mathrm{d} \cup \mathrm{d}$, via $\nabla\left(x_{0} \mathrm{~d} x_{1}\right)=x_{0} \varphi_{2}\left(x_{1}\right)$ for $x_{0} \in R^{+}, x_{1} \in R$. Then $\varphi_{2}$ raises the $M$-filtration degree by at most $a$. If $X$ is a filtered $R$-bimodule and $\psi: R \otimes R \rightarrow X$ is a 2-cocycle of degree at most $b$, then

$$
\bar{\psi}: \bar{\Omega}^{2} R \rightarrow X, \quad \bar{\psi}\left(x_{0} \mathrm{~d} x_{1} \mathrm{~d} x_{2}\right)=x_{0} \psi\left(x_{1}, x_{2}\right)
$$

is a bimodule homomorphism. And the 1-cochain

$$
\psi^{\prime}=\bar{\psi} \circ \varphi_{2}
$$

raises filtration degree by at most $a+b$ and satisfies $\delta\left(\psi^{\prime}\right)=\psi$. For $n \geq 1$, inductively define a 2-cocycle and a 1-cochain with values in $\bar{\Omega}^{2(n+1)} R$ as follows:

$$
\begin{aligned}
& \psi_{2(n+1)}:=\sum_{j=0}^{n} \mathrm{~d} \varphi_{2 j} \cup \mathrm{d} \varphi_{2(n-j)}-\sum_{j=1}^{n} \varphi_{2 j} \cup \varphi_{2(n+1-j)}, \\
& \varphi_{2(n+1)}:=\psi_{2(n+1)}^{\prime} .
\end{aligned}
$$

Put $\varphi_{0}=\mathrm{id}: R \rightarrow R$. To see that the maps $\psi_{2 n}$ are cocycles, one proves first that

$$
\delta\left(\mathrm{d} \varphi_{2 n}\right)=-\sum_{j=0}^{n} \mathrm{~d}\left(\varphi_{2 j} \cup \varphi_{2(n-j)}\right) .
$$

Then a long but straightforward calculation using the Leibniz rule for both $\mathrm{d}$ and $\delta$ shows by induction that $\delta\left(\psi_{2 n}\right)=0$ (see [10, Theorem 2.1]). By construction, the bounded linear map $\varphi_{\leq 2 n}:=\sum_{i=0}^{n} \varphi_{2 i}$ is a section of the canonical projection $\mathrm{T} R \rightarrow R$, and its curvature vanishes modulo $\mathrm{J} R^{n+1}$. So it defines a bounded algebra homomorphism $R \rightarrow \mathrm{T} R / \mathrm{J} R^{n+1}$. Hence the infinite series $\sum_{i=0}^{\infty} \varphi_{2 i}$ is an algebra homomorphism into the projective limit. It suffices to show that, for each $m$, the series $\sum_{i=0}^{\infty} \varphi_{2 i}$ defines a bounded linear homomorphism $R_{\mathrm{lg}} \rightarrow\left(\mathcal{U}\left(\mathrm{T} R_{\mathrm{lg}}, \mathrm{J} R_{\mathrm{lg}}^{m}\right), \mathcal{U}\left(\mathrm{J} R_{\mathrm{lg}}, \mathrm{J} R_{\mathrm{lg}}^{m}\right)\right)^{\dagger}$.

One checks by induction on $n$ that $\varphi_{2 n}\left(M^{(i)}\right) \subseteq \mathcal{F}_{i+(2 n-1) a}^{M} \bar{\Omega}^{2 n} R$. Hence

$$
\varphi_{2 n}\left(M^{\diamond}\right) \subseteq \sum_{i=0}^{\infty} \pi^{i} \mathcal{F}_{i+(2 n-1) a+1}^{M} \bar{\Omega}^{2 n} R .
$$

Next let $m \geq 1$ and choose an integer $c>\max \{1,2 a m\}$. Then

$$
i+\left\lfloor\frac{n}{m}\right\rfloor-\left\lceil\frac{i+(2 n-1) a+1}{c}\right\rceil \geq(1-1 / c) i \geq 0
$$


for all $i \geq 0$ and sufficiently large $n$. Then $i \geq\left\lceil\frac{i+(2 n-1) a+1}{c}\right\rceil-\left\lfloor\frac{n}{m}\right\rfloor$. Set $D(i, n, c):=$ $\left\lceil\frac{i+(2 n-1) a+1}{c}\right\rceil$. Equations 9.1.10 and 9.1.11 and Lemmas 9.1.4 and 9.1.5 imply

$$
\begin{aligned}
\varphi_{2 n}\left(M^{\diamond}\right) \subseteq \sum_{i \geq 0} \pi^{i} \mathcal{F}_{D(i, n, c)+2 n}^{M^{(c)}} \widetilde{\Omega}^{2 n}(R) \\
\\
\subseteq \pi^{-\left\lfloor\frac{n}{m}\right\rfloor} \sum_{i \geq 0} \pi^{D(i, n, c)} \mathcal{F}_{D(i, n, c)+2 n}^{M^{(c)}} \widetilde{\Omega}^{2 n}(R) \subseteq \pi^{-\left\lfloor\frac{n}{m}\right\rfloor} \widetilde{\Omega}^{2 n}\left(\left(M^{(c)}\right)^{\diamond}\right)
\end{aligned}
$$

By Proposition 4.4.15 the subset of infinite series $\sum_{n=0}^{\infty} \varphi_{2 n}\left(M^{\diamond}\right)$ is bounded in $\left(\mathcal{U}\left(\mathrm{T} R_{\mathrm{lg}}, \mathrm{J} R_{\mathrm{lg}}^{m}\right), \mathcal{U}\left(\mathrm{J} R_{\mathrm{lg}}, \mathrm{J} R_{\mathrm{lg}}^{m}\right)\right)^{\dagger}$. So $\sum_{n=0}^{\infty} \varphi_{2 n}$ defines a bounded homomorphism

$$
R \rightarrow\left(\mathcal{U}\left(\mathrm{T} R_{\lg }, \mathrm{J} R_{\lg }^{m}\right), \mathcal{U}\left(\mathrm{J} R_{\lg }, \mathrm{J} R_{\lg }^{m}\right)\right)^{\dagger}
$$

for each $m \geq 1$; this completes the proof.

Corollary 9.1.12. Let $R$ be as in Theorem 9.1.9. Then the natural map $\mathbb{H} \mathbb{A}\left(R^{\dagger}\right) \rightarrow X\left(R^{\dagger} \otimes F\right)$ is a chain homotopy equivalence and $\mathrm{HA}_{*}(R)$ is isomorphic to the homology of $X\left(R^{\dagger} \otimes F\right)$.

ProOF. Immediate from Theorem 9.1.9 and Corollary 4.7.2

9.2. Filtered Noetherian rings and smooth algebras. We now show that some quasi-free algebras have a connection of finite degree. In particular, this includes smooth, commutative finitely generated $V$-algebras of relative dimension 1 . For the remainder of this section, let $R$ be a finitely generated $V$-algebra, equipped with the fine bornology. Let $S \subseteq R$ be a finite generating subset and let $S^{\leq n}$ be the set of all products of elements of $S$ of length at most $n$. As above, let $\mathcal{F}_{n} R \subseteq R$ be the $V$-submodule generated by $S^{\leq n}$. By convention, $S^{\leq 0}=\{1\}$ and $\mathcal{F}_{0} R=V \cdot 1$. This is an increasing filtration on $R$. It induces filtrations on the bimodules $\Omega^{l}(R)$ as in Example 9.1.2 More concretely, $\mathcal{F}_{n}\left(\Omega^{l}(R)\right)$ is the $V$-submodule of $\Omega^{l}(R)$ generated by $x_{0} \mathrm{~d} x_{1} \ldots \mathrm{d} x_{l}$ with $x_{0} \in \mathcal{F}_{n_{0}}(R)$ or $x_{0}=1$ and $n_{0}=0$, and $x_{i} \in \mathcal{F}_{n_{i}}(R)$ for $i=1, \ldots, l$, and $n_{0}+\cdots+n_{l} \leq n$. By construction, the $V$-module $\mathcal{F}_{n} R \cdot \mathcal{F}_{m} R$ that is generated by products $x \cdot y$ with $x \in \mathcal{F}_{n} R, y \in \mathcal{F}_{m} R$ is equal to $\mathcal{F}_{n+m} R$ for all $n, m \in \mathbb{N}$. This is more than what is required for a filtered algebra, and the extra information is crucial for the filtration to generate the linear growth bornology.

Let $M$ be an $R$-module with a finite generating set $S_{M} \subseteq M$. Then we define a filtration on $M$, called the canonical filtration, by letting $\mathcal{F}_{n} M$ be the $V$-submodule generated by $a \cdot x$ with $a \in \mathcal{F}_{n} R$ and $x \in S_{M}$. This satisfies $\mathcal{F}_{m} R \cdot \mathcal{F}_{n} M \subseteq \mathcal{F}_{n+m} M$ because $\mathcal{F}_{m} R \cdot \mathcal{F}_{n} R \subseteq \mathcal{F}_{n+m} R$. The following proposition characterises canonical filtrations by a universal property:

Proposition 9.2.1. Let $R$ be a filtered $V$-algebra and let $M$ be a finitely generated $R$-module. Equip $M$ with the filtration described above. Then any $R$-module map from $M$ to a filtered $R$-module $Y$ is of finite degree. The canonical filtrations for two different finite generating sets of $M$ are shift equivalent.

Proof. Let $\left\{m_{1}, \ldots, m_{n}\right\}$ be a finite generating set for $M$ as an $R$-module. Let $h: M \rightarrow Y$ be an $R$-module homomorphism into a filtered $R$-module $Y$. Since $Y=\cup \mathcal{F}_{l} Y$, there is an $l \in \mathbb{N}$ with $h\left(m_{i}\right) \in \mathcal{F}_{l} Y$ for all $i=1, \ldots, m$. Then $h\left(a \cdot m_{i}\right) \in$ $\mathcal{F}_{n+l} R$ for $a \in \mathcal{F}_{n} R$. Hence $h\left(\mathcal{F}_{n} M\right) \subseteq \mathcal{F}_{n+l} Y$ for all $n \in \mathbb{N}$. That is, $h$ has finite degree. In particular, if we equip $M$ with another filtration $\left(\mathcal{F}_{n}^{\prime} M\right)_{n \in \mathbb{N}}$, then the identity map has finite degree, that is, there is $l \in \mathbb{N}$ with $\mathcal{F}_{n} M \subseteq \mathcal{F}_{n+l}^{\prime} M$ for all 
$n \in \mathbb{N}$. If the other filtration comes from another finite generating set, then we may reverse the roles and also get $l^{\prime} \in \mathbb{N}$ with inclusions $\mathcal{F}_{n}^{\prime} M \subseteq \mathcal{F}_{n+l^{\prime}} M$ for all $n \in \mathbb{N}$.

Definition 9.2.2. A filtered $V$-algebra $R$ is called (left) filtered Noetherian if every left ideal $I$ is finitely generated and the filtration $\left(\mathcal{F}_{n} R \cap I\right)_{n \in \mathbb{N}}$ is shift equivalent to the canonical filtration of Proposition 9.2.1 from a finite generating set. In other words, there are finitely many $x_{1}, \ldots, x_{n} \in I$ and $l \in \mathbb{N}$ such that for all $m \in \mathbb{N}$ and $y \in \mathcal{F}_{m} R \cap I$, there are $a_{i} \in \mathcal{F}_{m+l} R$ with $y=\sum_{i=1}^{n} a_{i} x_{i}$.

Lemma 9.2.3. Let $R$ be a finitely generated, quasi-free $V$-algebra. Assume that $R^{+} \otimes\left(R^{+}\right)^{\text {op }}$ is filtered Noetherian. Then $\Omega^{1}(R)$ has a connection of finite degree.

Proof. Since $R$ is quasi-free, the left multiplication map $R^{+} \otimes \Omega^{1}(R) \rightarrow \Omega^{1}(R)$ splits by an $R$-bimodule homomorphism $s: \Omega^{1}(R) \rightarrow R^{+} \otimes \Omega^{1}(R)$. By definition, $\Omega^{1}(R)$ is a left ideal in $R^{+} \otimes\left(R^{+}\right)^{\mathrm{op}}$. By assumption, it is finitely generated as such, and the filtration on $R^{+} \otimes\left(R^{+}\right)^{\text {op }}$ restricted to $\Omega^{1}(R)$ is the canonical filtration on $\Omega^{1}(R)$ as a module over $R^{+} \otimes\left(R^{+}\right)^{\text {op }}$. Now Proposition 9.2.1 shows that the section $s$ above has finite degree. The section $s$ yields a connection $\nabla: \Omega^{1}(R) \rightarrow \Omega^{2}(R)$, which is defined by $\nabla(\omega)=1 \otimes \omega-s(\omega)$. It follows that $\nabla$ has finite degree.

Our next goal is to show that a commutative, finitely generated $V$-algebra with the filtration coming from a finite generating set is filtered Noetherian. First consider the polynomial ring in $n$ variables. The filtration defined by the obvious generating set is the total degree filtration, where $\mathcal{F}_{m}\left(V\left[x_{1}, \ldots, x_{n}\right]\right)$ is the $V$-submodule generated by the monomials of total degree at most $m$, that is, terms of the form $x^{\alpha}=x_{1}^{\alpha_{1}} x_{2}^{\alpha_{2}} \cdots x_{n}^{\alpha_{n}}$ with $|\alpha|:=\sum_{i=1}^{n} \alpha_{i} \leq m$.

THEOREM 9.2.4. The polynomial ring $R=V\left[x_{1}, \ldots, x_{n}\right]$ with the total degree filtration is filtered Noetherian.

Proof. Let $I$ be any ideal in $R$. Since $R$ is Noetherian, $I$ is finitely generated. Since $V$ is a principal ideal domain, $I$ has a finite, strong Gröbner basis with respect to any term order on the monomials $x^{\alpha}$ (see $[\mathbf{2}$ Theorem 4.5.9]). We use the degree lexicographic order (see $[2$ Definition 1.4.3]); the only property we need is that $|\alpha|<|\beta|$ implies $x^{\alpha}<x^{\beta}$. The chosen order on monomials defines the leading term lt $(f)$ of a polynomial $f$. Let $G=\left\{f_{1}, \ldots, f_{N}\right\}$ be a strong Gröbner basis for $I$. By 2. Theorem 4.1.12], any $g \in I$ can be written as $g=\sum_{j=1}^{M} c_{j} t_{j} f_{i_{j}}$, where $M \in \mathbb{N}$, $c_{j} \in V, t_{j}$ is a monomial in $R, i_{j} \in\{1, \ldots, N\}$, and $\operatorname{lt}\left(t_{j} f_{i_{j}}\right)<\operatorname{lt}(g)$ for each $j$. So the total degree of $t_{j} f_{i_{j}}$ is at most the total degree of $g$ for each $j=1, \ldots, M$, and this remains so for the total degree of $t_{j}$. Combining the monomials $t_{j}$ with the same $i_{j}$, we write any element $g \in I$ of total degree at most $m$ in the form $\sum_{i=1}^{N} p_{j} f_{j}$ with $p_{j} \in \mathcal{F}_{m} R$.

Proposition 9.2.5. A quotient of a filtered Noetherian $V$-algebra with the induced filtration is again filtered Noetherian.

Proof. Let $R$ be a filtered Noetherian $V$-algebra and let $I$ be an ideal. Any ideal in the quotient ring $R / I$ is of the form $J / I$ for a unique ideal $J$ in $R$ containing $I$. Let $x_{1}, \ldots, x_{n} \in J$ and $l \in \mathbb{N}$ be such that for all $m \in \mathbb{N}$ and $y \in \mathcal{F}_{m} R \cap I$, there are $a_{i} \in \mathcal{F}_{m+l} R$ with $y=\sum_{i=1}^{n} a_{i} x_{i}$. Then the images of $x_{1}, \ldots, x_{n}$ in $J / I$ and the same $l$ will clearly work for the ideal $J / I$ in the quotient $R / I$. 
Corollary 9.2.6. Any finitely generated, commutative $V$-algebra is filtered Noetherian.

Proof. Let $A$ be a finitely generated, commutative $V$-algebra. Let $S$ be any finite generating set. Turn it into a surjective homomorphism from the polynomial algebra $R=V\left[x_{1}, \ldots, x_{n}\right]$ onto $A$. This identifies $A \cong R / I$ for an ideal $I$ in $R$. The filtration on $A$ defined by $S$ is equal to the filtration on the quotient $R / I$ defined by the degree filtration on $R$. Now the claim follows from Theorem 9.2.4 and Proposition 9.2.5.

Proposition 9.2.7. Let $R$ be a smooth, finitely generated commutative $V$-algebra of relative dimension 1 . Then $R$ admits a connection of finite degree.

ProOF. The assumptions on $R$ imply that $\Omega^{1}(R)$ a projective, finitely generated $R$-bimodule. Furthermore, by Corollary 9.2.6, $R$ is filtered Noetherian. The result now follows from Lemma 9.2.3.

REMARK 9.2.8. In their seminal article [28], Paul Monsky and Gerard Washnitzer introduced the so-called Monsky-Washnitzer cohomology $H_{\mathrm{MW}}^{*}(A)$ for a smooth unital $\mathbb{F}$-algebra $A$ that has a "very smooth" lift. This is a presentation $A=S / \pi S$ where $S$ is dagger complete and very smooth $([\mathbf{2 8}$, Definition 2.5$])$; by definition, $H_{\mathrm{MW}}^{*}(A)=H_{\mathrm{dR}}(S \otimes F)$ is the de Rham cohomology of $S \otimes F$. As in the current article, Monsky and Washnitzer assumed that $\operatorname{char}(F)=0$ but made no assumption about the characteristic of $\mathbb{F}$. The very smooth liftability assumption in 28] was crucial for their proof of the functoriality of $H_{\mathrm{MW}}^{*}$. Later on, Marius van der Put 32 managed to remove that assumption; for any smooth commutative unital $\mathbb{F}$-algebra $A$ of finite type, he defines $H_{\mathrm{MW}}^{*}(A)$ as the de Rham cohomology of the dagger completion of any smooth $V$-algebra $R$ with $R / \pi R=A$. The existence of such a lift follows from a theorem of Renée Elkik 18 ; van der Put proves functoriality of $H_{\mathrm{MW}}^{*}$ using Artin approximation. However, in his paper he assumes that $\mathbb{F}$ is finite. More recently, under very general assumptions (in particular, for $\mathbb{F}$ of arbitrary characteristic) Alberto Arabia $[3]$ proved that every smooth $\mathbb{F}$-algebra admits a very smooth lift, and extended the original definition of Monsky and Washnitzer. In a parallel development, Pierre Berthelot introduced rigid cohomology $H_{\text {rig }}^{*}(X)$ of general schemes $X$ over a field $\mathbb{F}$ with $\operatorname{char}(\mathbb{F})>0$, which for smooth affine $X=\operatorname{sp}(A)$ agrees with $H_{\mathrm{MW}}^{*}(A)$. With no assumptions on $\operatorname{char}(\mathbb{F})$, Große-Klönne $\mathbf{1 9}$ introduced the de Rham cohomology of dagger spaces over $V$, and he related it to rigid cohomology in the case when $\operatorname{char}(\mathbb{F})>0$.

The following is one of the main applications of our theory:

THEOREM 9.2.9. Let $X$ be a smooth affine variety over the residue field $\mathbb{F}$ of dimension 1 and let $A=\mathcal{O}(X)$ be its algebra of polynomial functions. Let $R$ be a smooth, commutative algebra of relative dimension 1 with $R / \pi R \cong A$. Equip $R$ with the fine bornology and let $R^{\dagger}$ be its dagger completion. If $*=0,1$, then $\mathrm{HA}_{*}\left(R^{\dagger}\right)$ is naturally isomorphic to the de Rham cohomology of $R^{\dagger}$. This is isomorphic to the Monsky-Washnitzer cohomology of $A$, which, if $\operatorname{char}(\mathbb{F})>0$, agrees with the rigid cohomology $H_{\text {rig }}^{*}(A, F)$ of $X$.

Proof. By our hypothesis and Proposition 9.2.7 $R$ is quasi-free. Equipping $R$ with the fine bornology, we are in the situation of Theorem 9.1.9. Then Corollary 9.1 .12 and $\left[\mathbf{1 1}\right.$, Theorem 5.5] imply that $\mathrm{HA}_{\star}\left(R^{\dagger}\right)$ is isomorphic to the de 
Rham cohomology of $R^{\dagger}$. Remark 9.2 .8 discusses the generality in which the latter is known to be isomorphic to different cohomology theories over $\mathbb{F}$.

Elkik [18 has shown that any smooth curve over $\mathbb{F}$ has a smooth lift over $V$. The following lemma shows that we may also arrange this lift to have relative dimension 1 as required in Theorem 9.2 .9

Lemma 9.2.10. Let $R$ be a smooth algebra, $A=R / \pi R$, and $d=\operatorname{dim} A$. Then $R=R_{1} \times R_{2}$, where $R_{1}$ is smooth of relative dimension $d$ and $R_{1} / \pi R_{1}=A$.

Proof. Since $R$ is smooth, $\Omega_{R / V}^{1}$ is projective. So its rank $r$ is a continuous function on $\operatorname{Spec}(R)$. Thus the set of primes $\mathfrak{P}$ where $r(\mathfrak{P})=d$ is clopen. This clopen subset induces a product decomposition $R \cong R_{1} \times R_{2}$. Since $\operatorname{dim} A=d$, the relative dimension is $d$ at all primes containing $\pi$. Now the lemma follows. 



\title{
CHAPTER 3
}

\section{A cyclic homology theory in positive characteristic}

\begin{abstract}
Let $V$ be a complete discrete valuation ring with uniformiser $\pi$ and residue field $\mathbb{F}=V / \pi V$. We define a cyclic homology theory for algebras over $\mathbb{F}$, which we call analytic cyclic homology, by lifting them to free algebras over $V$ and then building a tube algebra and completing to a dagger algebra. We show that this theory may be computed using any complete, torsion-free $V$-module lifting of an $\mathbb{F}$-algebra. We show that our theory is polynomially homotopy invariant, matricially stable, and satisfies excision.
\end{abstract}

\section{Introduction}

Cyclic homology and its variants have become fundamental invariants in noncommutative geometry. Developed by Connes and Tsygan in the 1980s, these invariants vastly generalise de Rham cohomology for smooth manifolds and schemes to non-commutative algebras. The approach of Cuntz and Quillen provided a new perspective in cyclic homology by introducing a non-commutative analogue of non-singular replacements of arbitrary algebraic varieties. Specifically, they build a certain universal nilpotent extension of an associative algebra $A$ over $\mathbb{C}$, by a quasi-free algebra $\mathcal{T}(A)$. From the viewpoint of cyclic homology, the consequence of this quasi-free replacement is that the periodic cyclic homology of $A$ can be computed by a much simpler chain complex - the $X$-complex of $\mathcal{T}(A)$. So a quasifree replacement plays the same role in cyclic homology that a smooth embedding does in de Rham cohomology. The use of suitably defined nilpotent extensions has thereafter become a standard approach in the definition of local, analytic and equivariant cyclic homology theories.

The robustness of the Cuntz-Quillen approach leads us to the natural question of extending periodic cyclic homology to the setting of non-Archimedean algebras. The ultimate aim of the program - which is far from complete - is that our homology theory specialises to rigid cohomology in the commutative setting. Our motivation comes from recent work in [1], which links Berthelot's rigid cohomology for commutative $\mathbb{F}_{p}$-algebras to the periodic cyclic homology of a certain dagger completed $\mathbb{Z}_{p}$-algebra lifting.

As a first step towards a theory for noncommutative $\mathbb{F}_{p}$-algebras, we introduced analytic cyclic homology in $\mathbf{1 2}$ for torsion-free non-Archimedean bornological algebras. We briefly recall its definition here. Let $V$ be a complete discrete valuation ring with uniformiser $\pi$, residue field $\mathbb{F}$ and fraction field $F$ of characteristic 0 . Given a complete, bornologically torsion-free $V$-algebra $R$, we use the Cuntz-Quillen machinery to build an analytically nilpotent extension

$$
\mathcal{J} R \nrightarrow \mathcal{T} R \rightarrow R
$$


with an analytically quasi-free algebra $\mathcal{T} R$. We define the analytic cyclic complex $\mathbb{H} \mathbb{A}(R)$ of $R$ as the projective system of $X$-complexes of $\mathcal{T} R \bar{\otimes} F$. These are projective systems of $\mathbb{Z}_{2}$-graded bornological $F$-vector spaces. We show that our homology theory is homotopy invariant for dagger homotopies, matricially stable and satisfies excision for semi-split extensions. In one of our main applications, we show that if $R$ is a smooth algebra of relative dimension 1 , then its analytic cyclic homology can be identified with the rigid cohomology of $R / \pi R$ with coefficients in $F$, when $\operatorname{char} F=0$.

We assume throughout this paper that $\operatorname{char} F=0$.

In this article, we define analytic cyclic homology for $\mathbb{F}$-algebras by lifting them to projective systems of torsion-free $V$-algebras (briefly, pro-algebras). We show that our theory is homotopy invariant for polynomial homotopies. Furthermore, we use the analytic cyclic theory in $\mathbf{1 2}$ to show that our theory for $\mathbb{F}$-algebras is matricially stable and satisfies excision for extensions of finitely generated algebras. These properties are desirable from the perspective of algebraic bivariant $K$-theory $([\mathbf{1 4}])$, which is the universal excisive, homotopy invariant, matricially stable functor from a category of algebras into a triangulated category. In our most important result, we show that different choices of complete, bornological torsion-free $V$-module liftings all compute the same theory.

The paper is organised as follows:

In Section 2, we define the analytic cyclic homology complex $\mathbb{H} \mathbb{A}(A, W, \varrho)$ of an $\mathbb{F}$-algebra $A$ with respect to a complete, bornologically torsion-free $V$-module lift $W \stackrel{\varrho}{\rightarrow} A$. It is defined as the $X$-complex of a pro-algebra, built from the tensor algebra $\mathrm{T} W$ and powers of the ideal $I:=\operatorname{ker}(\mathrm{T} W \rightarrow A)$. We refer to the analytic cyclic complex complex of $A$ with respect to the free $V$-module lift $V A \rightarrow A$ as 'the' analytic cyclic homology complex of $A$ and denote it by $\mathbb{H} \mathbb{A}(A)$. This is a projective system of $\mathbb{Z}_{2}$-graded chain complexes of bornological $F$-vector spaces. The homology $\mathrm{HA}_{*}(A)$ of a certain bornological completion of the homotopy inverse limit of $\mathbb{H} \mathbb{A}(A)$ is called the analytic cyclic homology of $A$.

In Section 3 we recall the simplified axioms of an exact category, following [21]. We need this generality to define the correct derived category in which different choices of torsion-free liftings of an algebra over $\mathbb{F}$ yield quasi-isomorphic chain complexes. We call an extension

$$
K \nrightarrow E \stackrel{p}{\rightarrow} Q
$$

in the category of bornological $V$-modules $\mathfrak{B}$ locally split if for every bounded submodule $M \subseteq Q$, there is a bounded $V$-linear section for $p$. We also define locally split extensions in the category $\overleftarrow{\mathfrak{B}}$ of projective systems of bornological $V$-modules. The class of locally split extensions are exact category structures on $\mathfrak{B}$ and $\overleftarrow{\mathfrak{B}}$. We use these exact category structures to define quasi-isomorphisms in the homotopy category of chain complexes $\operatorname{HoKom}(\overleftarrow{\mathfrak{B}})$ over $\overleftarrow{\mathfrak{B}}$. The derived category of the exact category $\overleftarrow{\mathfrak{B}}$ is defined as the localisation of $\operatorname{HoKom}(\overleftarrow{\mathfrak{B}})$ at the quasi-isomorphisms.

In Section 4 we use the exact category framework defined in Section 3 to show that for any complete, bornologically torsion-free $V$-module lifting $W \rightarrow A$, the complexes $\mathbb{H} \mathbb{A}(A, W, \varrho)$ and $\mathbb{H} \mathbb{A}(A)$ are quasi-isomorphic.

Sections 5 and 6 are about formal properties of our theory. We show that analytic cyclic homology is homotopy invariant for polynomial homotopies. We also show that if $\Lambda$ is a set and $M_{\Lambda}$ is the algebra of finitely supported matrices over $\mathbb{F}$, 
then the canonical map

$$
A \rightarrow A \otimes_{\mathbb{F}} M_{\Lambda}
$$

induces a quasi-isomorphism $\mathbb{H} \mathbb{A}(A) \cong \mathbb{H} \mathbb{A}\left(A \otimes_{\mathbb{F}} M_{\Lambda}\right)$. Finally, we show that there is a diagram of extensions

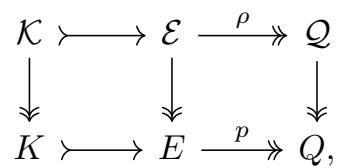

where the top row is a semi-split extension of pro-dagger algebras that surjects onto the bottow row of finitely generated $\mathbb{F}$-algebras with the fine bornology. This is used to show that analytic cyclic homology satisfies excision in the sense that if $K \nrightarrow E \rightarrow Q$ is an extension of finitely generated $\mathbb{F}$-algebras, then there is a natural 6 -term long exact sequence in homology

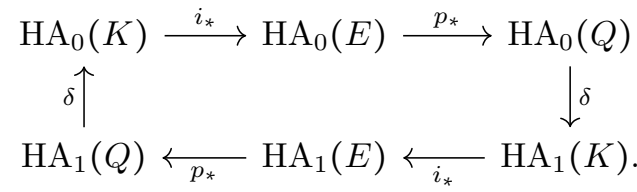

\section{Analytic cyclic theory in mixed characteristic}

In this section, we define analytic cyclic homology for $\mathbb{F}$-algebras. The definition follows an approach similar to that introduced in $\mathbf{1 2}$. Namely, we lift an $\mathbb{F}$-algebra to a suitably defined tensor algebra, which has desirable homological properties in the sense of Cuntz and Quillen. For an $\mathbb{F}$-algebra $A$ that admits a dagger algebra lifting $D$ - which is the situation considered in Monsky-Washnitzer cohomology the analytic cyclic homology of $A$ coincides with the analytic cyclic homology of $D$. Therefore, the definition we introduce is the right passage from torsion-free $V$-algebras to $\mathbb{F}$-algebras.

Definition 2.0.1. Let $W$ be a complete bornological $V$-module. Its (incomplete) tensor algebra TW is the direct sum $\oplus_{n=1}^{\infty} W^{\otimes n}$ with its canonical bornology and with the multiplication defined by

$$
\left(x_{1} \otimes \cdots \otimes x_{n}\right) \cdot\left(x_{n+1} \otimes \cdots \otimes x_{n+m}\right):=x_{1} \otimes \cdots \otimes x_{n+m} .
$$

Let $\sigma_{W}: W \rightarrow \mathrm{T} W$ be the inclusion of the first summand. It is a bounded $V$-module homomorphism, but not an algebra homomorphism.

A subset $S \subseteq \mathrm{T} W$ is bounded if and only if there are $a \in \mathbb{N}$ and a bounded, $\pi$-adically complete $V$-submodule $B \subseteq W$ such that $S$ is contained in the image in TW of $\sum_{n=1}^{a} B^{\otimes n}$. The tensor algebra has the following universal property:

LEMma 2.0.2. Let $S$ be a bornological $V$-algebra. Composition with $\sigma_{W}$ is a bijection from bounded homomorphisms $\mathrm{T} W \rightarrow S$ to bounded $V$-module maps $W \rightarrow S$.

Proof. Let $f: W \rightarrow S$ be a bounded $V$-module homomorphism. Then there is a unique bounded homomorphism $f^{\#}: \mathrm{T} W \rightarrow S$ with $f^{\#} \circ \sigma_{W}=f$, which is defined by

$$
f^{\#}\left(x_{1} \otimes \cdots \otimes x_{n}\right):=f\left(x_{1}\right) \cdots f\left(x_{n}\right)
$$


for all $x_{1}, \ldots, x_{n} \in W$.

In what follows, we go through a sequence of steps as in [12 Section 3], which leads to the definition of our homology theory.

The first step. Let $A$ be an $\mathbb{F}$-algebra and let $W$ be a torsion-free, complete bornological $V$-module with a surjective map $\varrho: W \rightarrow A$. We assume that $\varrho(M) \subseteq A$ is finite-dimensional for each bounded $V$-submodule $M \subseteq W$. Equivalently, $\varrho$ is bounded when $A$ carries the fine bornology. Let $R:=\mathrm{T} W$; this is a torsion-free bornological $V$-algebra. It is torsion-free because $W$ is torsion-free, and torsionfreeness is inherited by $n$-fold tensor products and direct sums. Since $A$ with the fine bornology is a bornological $V$-algebra, Lemma 2.0.2 also shows that $\varrho$ induces a bounded $V$-algebra homomorphism $\varrho^{\#}: R=\mathrm{T} W \rightarrow A$. Let $I:=\operatorname{ker} \varrho^{\#}$. Then we have a torsion-free $V$-algebra resolution

$$
I \nrightarrow R \rightarrow A
$$

of $A$.

The second step. We have built a torsion-free bornological $V$-algebra $R$ with an ideal $I$. As in the case of our homology theory for complete, bornologically torsion-free algebras, we enlarge $R$ to tube algebras for powers of $I$ in $R$. We recall their definition:

Definition 2.0.3. Let $R$ be a torsion-free bornological $V$-algebra and $I$ an ideal in $R$. Let $I^{j}$ for $j \in \mathbb{N}^{*}$ denote the $V$-linear span of products $x_{1} \cdots x_{j}$ with $x_{1}, \ldots, x_{j} \in I$. The tube algebra of $I^{l} \triangleleft R$ for $l \in \mathbb{N}^{*}$ is defined as

$$
\mathcal{U}\left(R, I^{l}\right):=\sum_{j=0}^{\infty} \pi^{-j} I^{l \cdot j} \subseteq R \otimes F,
$$

equipped with the subspace bornology; this is indeed a $V$-subalgebra of $R \otimes F$. If $l \geq j$, then $\mathcal{U}\left(R, I^{l}\right) \subseteq \mathcal{U}\left(R, I^{j}\right)$ is a bornological subalgebra. Let $\mathcal{U}\left(R, I^{\infty}\right)$ denote the resulting projective system of bornological $V$-algebras $\left(\mathcal{U}\left(R, I^{l}\right)\right)_{l \in \mathbb{N}^{*}}$.

Since $\mathcal{U}\left(R, I^{l}\right)$ is a bornological submodule of an $F$-vector space, it is bornologically torsion-free. Furthermore, the inclusion $R \hookrightarrow \mathcal{U}\left(R, I^{l}\right)$ induces a bornological isomorphism $\mathcal{U}\left(R, I^{l}\right) \otimes F \cong R \otimes F$.

The third step. In this step, we equip each tube-tensor algebra $\mathcal{U}\left(\mathrm{T} W, I^{l}\right)$ for $l \in \mathbb{N}^{*}$, with the relative linear growth bornology with respect to the ideal $\mathcal{U}\left(I, I^{l}\right)$ (see 12 Definition 2.2.3]). Since $\pi \cdot \mathcal{U}\left(R, I^{l}\right) \subseteq \mathcal{U}\left(I, I^{l}\right)$, the quotient $\mathcal{U}\left(R, I^{l}\right) / \mathcal{U}\left(I, I^{l}\right)$ is semi-dagger. So by [12 Lemma 2.26], there is no difference between the relative and the absolute linear growth bornologies on $\mathcal{U}\left(R, I^{l}\right)$.

The fourth step. Tensor with $F$. This gives a projective system of bornological $F$-algebras

$$
\mathcal{U}\left(R, I^{\infty}\right)_{\lg } \otimes F:=\left(\mathcal{U}\left(R, I^{l}\right)_{\lg } \otimes F\right)_{l \in \mathbb{N}^{*}} .
$$

The fifth step. In this step, we take the $X$-complex, defined for incomplete algebras as follows:

Definition 2.0.4. Let $S$ be a bornological $V$-algebra. The $X$-complex of $S$ is defined as

$$
X(S):=\left(S \underset{\tilde{b}}{\stackrel{q \circ \mathrm{d}}{\rightleftarrows}} \Omega^{1}(S) /[\cdot, \cdot]\right)
$$


where $\Omega^{1}(S) /[\cdot, \cdot]$ denotes the quotient of the noncommutative 1 -forms by the cokernel of the map

$$
S \otimes \Omega^{1}(S) \rightarrow \Omega^{1}(S), \quad x \otimes \omega \mapsto x \cdot \omega-\omega \cdot x .
$$

The maps $q$, d and $\tilde{b}$ are the same maps already defined in [12 Section 2.7].

In our case, we take the $X$-complex of the projective system of incomplete bornological algebras $\mathcal{U}\left(R, I^{\infty}\right)_{\lg } \otimes F$. These define a projective system of $\mathbb{Z} / 2$-graded chain complexes of bornological $F$-vector spaces, which we call the analytic cyclic complex $\mathbb{H} \mathbb{A}(A ; W, \varrho)$ of $A$ with respect to the lifting $\varrho: W \rightarrow A$.

The sixth step. The next step takes the homotopy inverse limit holim $\mathbb{H} \mathbb{A}(A, W, \varrho)$ of the analytic chain complex. More explicitly, this is the mapping cone of the chain map

$$
\begin{aligned}
\left.\prod_{l \in \mathbb{N}^{*}} X\left(\mathcal{U}\left(R, I^{l}\right)\right) \otimes F\right) & \rightarrow \prod_{l \in \mathbb{N}^{*}} X\left(\mathcal{U}\left(R, I^{l}\right) \otimes F\right), \\
\left(x_{l}\right) & \mapsto\left(x_{l}-\sigma_{l}\left(x_{l+1}\right)\right)_{l \in \mathbb{N}^{*}} .
\end{aligned}
$$

We will discuss the homotopy inverse limit construction in greater depth in Section 3 This gives us a $\mathbb{Z} / 2$-graded chain complex of bornological $F$-vector spaces.

The seventh step. Finally, we impart a notion of completion to our bornological structures that has better exactness properties. Recall that we can explicitly describe the completion of a bornological $V$-module as follows: given a bornological $V$-module $M=\lim M_{i}$, its completion is defined as $\overparen{M}=\lim \widehat{M}_{i} / \overline{\{0\}}$. The functor that takes a bornological $V$-module to the quotient by the bornological closure of the trivial module $\{0\}$ is called the separated quotient functor. In general, this functor is not exact and the separated quotient of a bornological $V$-module is hard to describe explicitly. This is also why we delay the process of completion; instead of directly taking the dagger completion of the tube-tensor algebra in step 2 as in $\mathbf{1 1} \mathbf{1 2}$. More specifically, the definition of analytic cyclic homology involves a certain analytic tensor algebra lifting $\mathcal{T} R$ of a complete, bornologically torsion-free $V$-algebra $R$. We described this tensor algebra explicitly in $\mathbf{1 2}$, Section 4.4], using the bornology of $R$ and subcompleteness of the tensor product (see $\mathbf{1 2}$. Proposition 2.4.5]). However, to associate a natural chain complex to an $\mathbb{F}$-algebra $A$, we need to use free algebra liftings in place of tensor algebras, for which the separated quotient functor is hard to control. Therefore, we replace the completion functor with the quasi-completion of a bornological $V$-module:

Definition 2.0.5. Let $M$ be a bornological $V$-module written as an inductive limit $M \cong \lim _{\longrightarrow} M_{i}$ of the directed set of its bounded $V$-submodules $M_{i}$. The quasicompletion of $M$ is defined as $\mathcal{Q}(M):=\underline{\lim } \widehat{M}_{i}$. It is a bornological $V$-module with the inductive limit bornology.

We now take the quasi-completion of the homotopy inverse limit of $\mathbb{H} \mathbb{A}(A, W, \varrho)$ at each degree. This yields a $\mathbb{Z}_{2}$-graded bornological chain complex.

The eighth and the last step. In what follows, let $A$ and $\varrho: W \rightarrow A$ be as in Step 1.

Definition 2.0.6. The analytic cyclic homology of an $\mathbb{F}$-algebra $A$ with respect to $W$ is the homology $\operatorname{HA}_{*}(A, W, \varrho)$ of the chain complex $\mathcal{Q}(\operatorname{holim}(\mathbb{H} \mathbb{A}(A, W, \varrho)))$, that is, the quotient of the kernel of the differential by the image of the differential, equipped with the induced bornology. 
REMARK 2.0.7. The definitions above also work if the lifting $W$ of an $\mathbb{F}$-algebra is a projective system of $V$-modules. We then need a tube algebra that is a projective system over the powers of the ideal as well as over the indexing set of the pro-tensor algebra TW (see [12 Section 4.2]). We still denote the analytic cyclic complex of a pro- $V$-module lifting $W \rightarrow A$ by $\mathbb{H} \mathbb{A}(A, W, \varrho)$.

The following result formulates the functoriality of our homology theory:

Lemma 2.0.8. For $j=1,2$, let $A_{j}$ be $\mathbb{F}$-algebras with the fine bornology, let $W_{j}$ be torsion-free, complete bornological $V$-modules with bounded $V$-module maps $\varrho_{j}: W_{j} \rightarrow A_{j}$. Let $g: W_{1} \rightarrow W_{2}$ be a bounded $V$-module homomorphism that satisfies $g\left(\operatorname{ker} \varrho_{1}\right) \subseteq \operatorname{ker} \varrho_{2}$. Then $g$ induces a bounded F-linear map $\mathrm{HA}_{*}\left(A_{1} ; W_{1}, \varrho_{1}\right) \rightarrow$ $\mathrm{HA}_{*}\left(A_{2} ; W_{2}, \varrho_{2}\right)$.

Proof. The map $g: W_{1} \rightarrow W_{2}$ induces a bounded $V$-algebra homomorphism

$$
\mathrm{T}(g): \mathrm{T} W_{1} \rightarrow \mathrm{T} W_{2} .
$$

Furthermore, the condition $g\left(\operatorname{ker} \varrho_{1}\right) \subseteq \operatorname{ker} \varrho_{2}$ means that the $\mathbb{F}$-linear map $A_{1} \rightarrow A_{2}$, $\tilde{g}: x+\operatorname{ker}\left(\varrho_{1}\right) \mapsto g(x)+\operatorname{ker}\left(\varrho_{2}\right)$ is well-defined. Finally, the maps $\varrho_{j}: W_{j} \rightarrow A_{j}$ induce bounded $V$-algebra homomorphisms $\varrho_{j}^{*}: \mathrm{T} W_{j} \rightarrow A_{j}$ such that $\varrho_{2}^{*} \circ \mathrm{T}(g)=\tilde{g} \circ \varrho_{1}^{*}$. So $\mathrm{T}(g)$ maps $I_{1}:=\operatorname{ker}\left(\mathrm{T} W_{1} \rightarrow A_{1}\right)$ to $I_{2}:=\operatorname{ker}\left(\mathrm{T} W_{2} \rightarrow A_{2}\right)$. Hence $\mathrm{T}(g)$ induces a map $\mathcal{U}\left(\mathrm{T} W_{1}, I_{1}^{l}\right) \rightarrow \mathcal{U}\left(\mathrm{T} W_{2}, I_{2}^{l}\right)$ for each $l$. Since tensor product with $F$, the $X$ complex, homotopy projective limits, and quasi-completion are functors, we obtain a chain map $\mathcal{Q}\left(\operatorname{holim}\left(\mathbb{H} \mathbb{A}\left(A_{1}, W_{1}, \varrho_{1}\right)\right)\right) \rightarrow \mathcal{Q}\left(\operatorname{holim}\left(\mathbb{H} \mathbb{A}\left(A_{2}, W_{2}, \varrho_{2}\right)\right)\right)$. It induces a map in homology $\mathrm{HA}_{*}\left(A_{1}, W_{1}, \varrho_{1}\right) \rightarrow \mathrm{HA}_{*}\left(A_{2}, W_{2}, \varrho_{2}\right)$ for each $*=0,1$.

2.1. Special lifts of an algebra over the residue field. We now consider two special types of liftings that arise in practice.

Monsky-Washnitzer type lifting. The situation in Monsky-Washnitzer cohomology is as follows: consider a smooth, unital $\mathbb{F}$-algebra $A$ with a weakly complete, "very smooth" $V$-algebra $D$ that satisfies $D / \pi D \cong A$. In our setup, this means that if we equip $A$ with the fine bornology, then there is a resolution by a dagger algebra $\pi D \nrightarrow D \rightarrow A$. We can now apply our machinery and define the analytic cyclic homology of $A$ with respect to its dagger algebra lifting $D$. This turns out to be the same as the analytic cyclic homology of $D$ as defined in [12 Section 3]. Importantly, there is no difference between the quasi-completion and the completion of the tube-tensor algebra $\mathcal{U}\left(\mathrm{T} D, \mathrm{~J} D^{m}\right)$ in the linear growth bornology.

Proposition 2.1.1. Let $D$ be a dagger algebra with the property that the quotient bornology on $D / \pi D$ is the fine bornology. Then $\mathbb{H} \mathbb{A}(D / \pi D, D, q)$ is quasi-isomorphic to the analytic cyclic homology complex $\mathbb{H} \mathbb{A}(D)$ of the dagger algebra $D$.

Proof. Let $A:=D / \pi D$. We claim that the ideals $\mathrm{J} D$ and

$$
\operatorname{ker}(\mathrm{T} D \rightarrow A)=\mathrm{J} D \oplus \pi \cdot \sigma_{D}(D)=\mathrm{J} D+\pi \cdot \mathrm{T} D
$$

in $\mathrm{T} D$ generate the same tube algebras. To check the claim, let $l \in \mathbb{N}_{\geq 1}$ and compute

$$
\sum_{a=0}^{\infty} \pi^{-a}(\mathrm{~J} D+\pi \cdot \mathrm{T} D)^{a l} \subseteq \sum_{a=0}^{\infty} \sum_{i=0}^{a l} \pi^{-a+i}(\mathrm{~J} D)^{a l-i}=\sum_{a=0}^{\infty} \pi^{-a}(\mathrm{~J} D)^{a l} .
$$

So the tube algebras defining $\mathbb{H} \mathbb{A}(D)$ and $\mathbb{H} \mathbb{A}(D / \pi D, D, q)$ are the same. We now show that quasi-completion commutes with homotopy inverse limits. To show this, we will need the following: 
LEMMA 2.1.2. Quasi-completions commute with countable products.

PROOF. Let $\left(M_{n}\right)_{n \in \mathbb{N}}$ be a collection of bornological $V$-modules and let $M=$ $\prod_{n \in \mathbb{N}} M_{n}$. Writing each $M_{n}$ as an inductive limit $M_{n}=\lim _{i_{n} \in I_{n}} M_{n, i_{n}}$, we can describe the bornology on $M$ explcitly as follows: a subset in $M$ is bounded if and only if it is contained in some bounded submodule of the form $\prod_{n \in \mathbb{N}} M_{n, i_{n}}$, for $i_{n} \in I_{n}$. So the quasi-completion of $M$ is the inductive limit of $\prod_{n \in \mathbb{N}}{\overline{M_{n, i_{n}}}}_{\text {. This }}$ is also precisely the product of the quasi-completions $\mathcal{Q}\left(M_{n}\right)$.

It remains to show that the quasi-completion of the complex $X\left(\mathcal{U}\left(\mathrm{T} D, \mathrm{~J} D^{\infty}\right)_{\lg } \otimes\right.$ $F)$ is isomorphic to the completed analytic chain complex $\bar{X}(\mathcal{T} D \otimes F)$ that defines the analytic cyclic homology of the dagger algebra $D$. Let $l \in \mathbb{N}^{*}$ be fixed. In degree 0 , the $X$-complex is $\mathcal{U}\left(\mathrm{T} D, \mathrm{~J} D^{l}\right)_{\lg } \otimes F$, and in degree 1 it is the commutator quotient of $\Omega^{1}\left(\mathcal{U}\left(\mathrm{T} D, \mathrm{~J} D^{l}\right)_{\lg } \otimes F\right)$. Here by commutator quotient, we mean the quotient by the image of the map $\mathcal{U}\left(\mathrm{T} D, \mathrm{~J} D^{l}\right)_{\lg } \otimes \Omega^{1}\left(\mathcal{U}\left(\mathrm{T} D, \mathrm{~J} D^{l}\right)_{\lg }\right) \rightarrow \Omega^{1}\left(\mathcal{U}\left(\mathrm{T} D, \mathrm{~J} D^{l}\right)_{\lg }\right)$, $x \otimes \omega \mapsto x \cdot \omega-\omega \cdot x$.

We first observe that submodules of the form $\oplus_{j=0}^{\infty} \pi^{-\left\lfloor\frac{j}{l}\right\rfloor} \Omega^{2 j}(M)$, for bounded $\pi$ adically complete submodules of $D$, cofinally generate a bornology that is isomorphic to the linear growth bornology of the tube algebra $\mathcal{U}\left(\mathrm{T} D, \mathrm{~J} D^{l}\right)_{\lg }$ (see $\mathbf{1 2}$, Corollary 4.1.4]). Now let $M \hookrightarrow N$ be a bornological embedding between bounded, $\pi$-adically complete submodules of $D$. Then by [12 Proposition 2.4.5], we have an inclusion $\widetilde{\Omega}^{1}(M) \subseteq \widetilde{\Omega}^{1}(N)$. So we have $\prod_{n=0}^{\infty} \pi^{-\left\lfloor\frac{n}{l}\right\rfloor} \Omega^{2 n}(M) \hookrightarrow \prod_{n=0}^{\infty} \pi^{-\left\lfloor\frac{n}{l}\right\rfloor} \widetilde{\Omega}^{2 n}(N)$, which implies the separatedness of the inductive limit. Varying $l$, we can identify $\Omega^{1}\left(\mathcal{U}\left(\mathrm{T} D, \mathrm{~J} D^{\infty}\right)_{\mathrm{lg}}\right) /[\cdot, \cdot]$ with the pro-bimodule differential forms of odd degree. So we can repeat the same argument as above to deduce that the quasi-completion of this pro-module is isomorphic to $\widetilde{\Omega}^{1}\left(\mathcal{U}\left(\mathrm{T} D, \mathrm{~J} D^{\infty}\right)^{\dagger}\right) / \overline{[\cdot, \cdot]}$.

Corollary 2.1.3. Let $A$ be the coordinate ring of a smooth, affine variety $X$ over $\mathbb{F}$ of relative dimension 1 . Suppose $R$ is a smooth, commutative $V$-algebra with the fine bornology, satisfying $R / \pi R \cong A$. Then for $*=0,1$, the following homology groups are isomorphic at each degree:

(1) $\mathrm{HA}_{*}\left(A, R^{\dagger}, R^{\dagger} \rightarrow A\right)$;

(2) the de Rham cohomology of $R^{\dagger} \otimes F$;

(3) the Monsky-Washnitzer cohomology of $X$;

(4) if char $(\mathbb{F})>0$, then the rigid cohomology $H_{\text {rig }}^{*}(A, F)$ of $X$.

Proof. The homology groups in (2), (3) and (4) are all isomorphic to $\mathrm{HA}_{*}\left(R^{\dagger}\right)$ by $\mathbf{1 2}$, Theorem 8.2.9].

We would like to show that the homology theories above are isomorphic to $\mathrm{HA}_{*}(A)$, which is our definition for the analytic cyclic homology for an $\mathbb{F}$-algebra. This is true by Proposition 2.1.8, which we prove in the Section 4

Pro-dagger algebra lift with pro-nilpotent kernel. We end this section with a generalisation of Proposition 2.1.1. This generalisation will be needed to prove excision in Section 6 Recall that a pro-algebra $N$ is called analytically nilpotent if it is isomorphic to a projective system of dagger algebras, and is nilpotent mod $\pi$ (see [12, Definition 4.3.1]).

Proposition 2.1.4. Let $A$ be an $\mathbb{F}$-algebra with the fine bornology, viewed as a constant projective system. Let $I_{n} \rightarrow D_{n} \stackrel{\varrho_{n}}{\rightarrow} A$ be an extension of pro-algebras, where 
$D=\left(D_{n}\right)_{n \in \mathbb{N}}$ is a pro-dagger algebra and $I=\left(I_{n}\right)_{n \in \mathbb{N}}$ is analytically nilpotent. Denote the surjections $\left(\varrho_{n}: D_{n} \rightarrow A\right)_{n \in \mathbb{N}}$ by $\varrho: D \rightarrow A$. Then $\mathbb{H} \mathbb{A}(A, D, \varrho)$ is quasi-isomorphic to $\mathbb{H} \mathbb{A}(D)$.

Proof. Let $J=\operatorname{ker}(\mathrm{T} D \rightarrow A)$ be the pro-algebra $J_{n}=\operatorname{ker}\left(\mathrm{T} D_{n} \rightarrow A\right)$. By hypothesis, for each $n$, there are $m \geq n$ and $k \geq 1$ such that $I_{m}^{k} \subseteq \pi \cdot I_{n}$. So the image of $J_{m}^{k}$ is contained in $J D_{n} \oplus \pi I_{n}$. By a computation as in the proof of Proposition 2.1.1, it follows that for each $n$, there are $m \geq n$ and $l=j k \geq j$, such that the image of $\mathcal{U}\left(\mathrm{T} D_{m}, J_{m}^{l}\right)$ is contained in $\mathcal{U}\left(\mathrm{T} D_{n}, \mathrm{~J} D_{n}^{j}\right)$. And the image of $\mathcal{U}\left(\mathrm{T} D_{n}, \mathrm{~J} D_{n}^{j}\right)$ is contained in $\mathcal{U}\left(\mathrm{T} D_{n}, J_{n}^{j}\right)$ for each $n \in \mathbb{N}$ and $j \in \mathbb{N}^{*}$. Therefore, the pro-algebras $\mathcal{U}\left(\mathrm{T} D, I^{\infty}\right)$ and $\mathcal{U}\left(\mathrm{T} D, \mathrm{~J} D^{\infty}\right)$ are isomorphic as projective systems. Applying the $X$-complex gives an isomorphism of projective systems of chain complexes $X\left(\mathcal{U}\left(\mathrm{T} D, I^{\infty}\right)\right) \rightarrow X\left(\mathcal{U}\left(\mathrm{T} D, \mathrm{~J} D^{\infty}\right)\right)$. Now the functoriality of homotopy inverse limits, quasi-completions and Proposition 2.1.1 imply that $\mathrm{HA}_{*}(D) \cong \mathrm{HA}_{*}(A, D, \varrho)$ for $*=0,1$.

Remark 2.1.5. Let $A$ be an $\mathbb{F}$-algebra and let $N \rightarrow D \rightarrow A$ be an extension by a pro-algebra $D$ and a nilpotent $\bmod \pi$ kernel $N$. Then $D$ is automatically isomorphic to a projective system of semi-dagger algebras. This is because by 12. Remark 4.22], the reduction $\bmod N / \pi N$ is nilpotent, and hence isomorphic to a projective system of semi-dagger algebras. Since $\pi N$ is also a projective system of semi-dagger algebras, so is $N$ by [12 Proposition 4.3.13]. Of course, since $\pi \cdot A=0$, $A$ is also semi-dagger. Viewing $A$ as a constant pro-algebra, we can again use 12. Proposition 4.3.13] to conclude that $D$ is isomorphic to a projective system of semi-dagger algebras.

2.1.1. The free $V$-algebra lifting of an $\mathbb{F}$-algebra. The approach in $1 \mathbf{1 1}$ uses the free commutative $V$-algebra lifting $V[A] \rightarrow A$ of a commutative $\mathbb{F}$-algebra $A$, and associates to it a functorial chain complex. If the algebra $A$ is finitely generated, their construction yields rigid cohomology. In our setting, the algebra $A$ is non-commutative. So we must replace the free commutative algebra by the free $V$-algebra $V\langle A\rangle$, defined as follows:

Definition 2.1.6. Let $S$ be a set. Let $\langle S\rangle$ denote the free semigroup generated by $S$; this is the set of all non-empty words with letters in $S$, with concatenation of words as multiplication. Let $V\langle S\rangle$ be the free $V$-module generated by $\langle S\rangle$, equipped with the multiplication induced by the product in $\langle S\rangle$. This is the free algebra generated by $S$. Namely, it has the following universal property: for any $V$-algebra $B$, there is a natural bijection between algebra homomorphisms $V\langle S\rangle \rightarrow B$ and maps $S \rightarrow B$. We write $f^{\#}: V\langle S\rangle \rightarrow B$ for the algebra homomorphism induced by a map $f: S \rightarrow B$.

If $S$ is a subset of $A$, then the inclusion map $i: S \rightarrow A$ induces a $V$-algebra homomorphism $i^{\#}: V\langle S\rangle \rightarrow A$. It is surjective if and only if $S$ generates $A$ as an $\mathbb{F}$-algebra. A functorial choice for the generating set is $S=A$.

Lemma 2.1.7. Let $S$ be a set and let $V S$ be the free $V$-module over $S$. Then $V\langle S\rangle \cong \mathrm{\top}(V S)$.

Proof. Let $B$ be a $V$-algebra and let $S \rightarrow B$ be a map. Since $B$ is in particular a $V$-module, the universal property of free modules, gives a unique $V$-linear map $V S \rightarrow B$ extending $S \rightarrow B$. Since $B$ is a $V$-algebra, the universal property of the 
tensor algebra gives a unique extension $\mathrm{T}(V S) \rightarrow B$. The claim now follows from the Yoneda Lemma.

We equip the free $V$-module $V A$ and the tensor algebra $R:=V\langle A\rangle \cong \mathrm{T}(V A)$ with the fine bornology. Then $V A$ and $R$ are both complete as $V$-modules. Since $V A$ is free, the algebra $R$ is torsion-free and since it has the fine bornology, it is also bornologically torsion-free. So we obtain a bornologically torsion-free resolution

$$
I \gg R \stackrel{p}{\rightarrow} A,
$$

which we feed into our machinery. We denote the resulting analytic chain complex by $\mathbb{H} \mathbb{A}(A):=\mathbb{H} \mathbb{A}(A, V A, V A \rightarrow A)$ and call the resulting homology $\mathrm{HA}_{*}(A)$ 'the' analytic cyclic homology of $A$. This name is justified in our main theorem, which we now formulate:

Proposition 2.1.8. Let $A$ be an $\mathbb{F}$-algebra, let $W$ be a complete, bornologically torsion-free bornological $V$-module, and let $\varrho: W \rightarrow A$ be a surjective $V$-module map that is bounded in the fine bornology on A. Assume that $\varrho$ has a bounded section. Then there is a canonical bornological F-module isomorphism

$$
\mathrm{HA}_{*}(A) \cong \mathrm{HA}_{*}(A ; W, \varrho) \text {. }
$$

Let $A, W$ and $\varrho: W \rightarrow A$ be as in Proposition 2.1.8 Let $I:=\operatorname{ker} \varrho^{\#}$. The homology $\mathrm{HA}_{*}(A ; W, \varrho)$ is based on the projective system of bornological $V$-algebras $\mathcal{U}\left(\mathrm{T} W, I^{l}\right)$ for $l \in \mathbb{N}_{\geq 1}$. The homology $\mathrm{HA}_{*}(A)$ is based on the projective system of bornological $V$-algebras $\mathcal{U}\left(\mathrm{T}(V A), J^{l}\right)$ for $l \in \mathbb{N}_{\geq 1}$, where $J:=\operatorname{ker}(p: \mathrm{T}(V A) \rightarrow A)$. We first build a natural map

$$
\mathrm{HA}_{*}(A) \rightarrow \mathrm{HA}_{*}(A ; W, \varrho) .
$$

The starting point is a map $s: A \rightarrow W$ with $\varrho \circ s=\operatorname{id}_{A}$. This induces a $V$-linear map $V A \rightarrow W$, which we compose with the canonical linear map $\sigma_{W}: W \rightarrow \mathrm{T} W$. The composite induces a homomorphism $s_{*}$ from $V\langle A\rangle=\mathrm{T}(V A) \rightarrow \mathrm{T} W$. It satisfies $\varrho^{\#} \circ s_{*}=p$ and hence maps $J$ to $I$. Then it extends uniquely to compatible homomorphisms $s_{\star}^{(l)}: \mathcal{U}\left(\mathrm{T}(V A), J^{l}\right) \rightarrow \mathcal{U}\left(\mathrm{T} W, I^{l}\right)$ for all $l \in \mathbb{N}$. The bornology on $\mathcal{U}\left(\mathrm{T}(V A), J^{l}\right)$ is the fine one, so that these homomorphisms are bounded. We get $\mathrm{HA}_{*}(A)$ and $\mathrm{HA}_{*}(A ; W, \varrho)$ by applying the steps $3-8$ in the definition of analytic cyclic homology to the projective systems of bornological algebras $\mathcal{U}\left(\mathrm{T}(V A), J^{l}\right)$ and $\mathcal{U}\left(T W, I^{l}\right)$. Since each of these steps is functorial, the family of homomorphisms $\left(s_{*}^{(l)}\right)_{l \in \mathbb{N}_{\geq 1}}$ induces a map $\mathrm{HA}_{*}(A) \rightarrow \mathrm{HA}_{*}(A ; W, \varrho)$. To show that this map is invertible, we first need to develop some machinery that is motivated by local cyclic homology [26 Section 2.3]. Using this machinery, we will construct 'local' algebra homomorphisms in the opposite direction that are inverse to $s_{*}^{(l)}$ in a suitable sense. We will ultimately show that the resulting chain complexes $\mathbb{H} \mathbb{A}(A)$ and $\mathbb{H} \mathbb{A}(A, W, \varrho)$ are 'locally' chain homotopy equivalent.

\section{The exact category of locally split extensions}

In this section, we define exact category structures on the categories of bornological $V$-modules and projective systems of bornological $V$-modules (over countable directed sets). Let $\mathcal{C}$ be an additive category $\mathcal{C}$ with kernels and cokernels. A diagram of the form

$$
K \stackrel{i}{\rightarrow} E \stackrel{p}{\rightarrow} Q
$$


is called an extension if $i=\operatorname{ker}(p)$ and $p=\operatorname{coker}(i)$. An exact category is an additive category $\mathcal{C}$ with a distinguished class of extensions $\mathcal{E}$ ) called conflations, satisfying certain properties. An arrow in $\mathcal{C}$ is called an inflation (respectively, deflation) if it is the arrow $i$ (respectively, $p$ ) in a conflation. The conflations must satisfy the following axioms:

- the identity map on the zero object is a deflation;

- if $A \stackrel{f}{\rightarrow} B$ and $B \stackrel{g}{\rightarrow} C$ are deflations, so is $A \stackrel{g \circ f}{\rightarrow} C$;

- the pullback of a deflation along an arbitrary map exists and is again a deflation;

- the pushout of an inflation along an arbitrary map exists and is again an inflation.

3.1. Locally split extensions of bornological modules. We now define the relevant exact category structures on the categories $\mathfrak{B}$ and $\overleftarrow{\mathfrak{B}}$ of torsionfree bornological $V$-modules and projective systems of torsion-free bornological $V$-modules, respectively. Briefly, we require extensions of bornological $V$-modules that split locally, that is, on each bounded $V$-submodule of the quotient.

Definition 3.1.1. An extension as in 3.0.1 of torsion-free bornological $V$ modules is called locally split if for any bounded $V$-submodule $M \subseteq Q$, there is a bounded $V$-module map $s: M \rightarrow E$ such that $p \circ s: M \rightarrow Q$ is the inclusion map. We call a bounded $V$-module map $X \stackrel{f}{\rightarrow} Q$ locally liftable into the extension (3.0.1) if for any bounded $V$-submodule $M \subseteq X$, there is a bounded $V$-module map $\hat{f}: M \rightarrow E$ such that $p \circ \hat{f}=f \circ \iota_{M}$, where $\iota_{M}$ is the inclusion $M \subseteq X$;

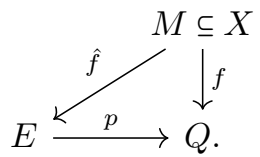

REMARK 3.1.2. An extension is locally split if and only if $\operatorname{id}_{Q}$ is locally liftable, if and only if any bounded $V$-module map $X \rightarrow Q$ is locally liftable.

In what follows, and throughout this article, we shall only consider projective systems indexed by countable directed sets $N$. We can actually assume $N=\mathbb{N}$. This is because restriction to cofinal subsets yields isomorphic projective systems.

Definition 3.1.3. An extension of projective systems of torsion-free bornological $V$-modules is called locally split if it is isomorphic to a projective system of extensions $\left(K_{n} \rightarrow E_{n} \rightarrow Q_{n}\right)_{n \in \mathbb{N}}$, where for each $n \in \mathbb{N}$, there exists $m \geq n$ such that the canonical map $Q_{m} \rightarrow Q_{n}$ is locally liftable with respect to the extension $K_{n} \nrightarrow E_{n} \rightarrow$ $Q_{n}$.

A few remarks are in order here. The proof of [12, Proposition 4.3.13] shows that an extension $K \nrightarrow E \rightarrow Q$ of projective systems of bornological $V$-modules is isomorphic to a projective system of extensions of bornological $V$-modules $\left(K_{n}^{\prime} \nrightarrow\right.$ $\left.E_{n}^{\prime} \rightarrow Q_{n}^{\prime}\right)_{n \in \mathbb{N}}$, such that $\left(K_{n}^{\prime}\right)_{n},\left(E_{n}^{\prime}\right)_{n}$ and $\left(Q_{n}^{\prime}\right)_{n}$ are isomorphic as projective systems to $K, E$ and $Q$, respectively. We do not assume that the local sections are compatible with the structure maps of $E$ and $Q$. Finally, it is easy to see that the definition of locally split extensions is independent of the choice of representation as a projective system of extensions. 
Denote by $\mathfrak{E}$ and $\overleftarrow{\mathfrak{E}}$ the classes of locally split extensions in the categories $\mathfrak{B}$ and $\overleftarrow{\mathfrak{B}}$, respectively.

REMARK 3.1.4. The class of locally split extensions defined above is inspired by the locally split extensions defined in [26. Section 2.3.6] for categories of projective and inductive systems over an additive category.

Lemma 3.1.5. The pair $(\mathfrak{B}, \mathfrak{E})$ is an exact category.

Proof. Clearly, the zero map on the zero module is a deflation. To see that the composition of two deflations $p: A \rightarrow B$ and $q: B \rightarrow C$ is a deflation, let $M$ be a bounded $V$-submodule of $C$. Then there exists a bounded $V$-module map $s_{M}: M \rightarrow B$ such that $q \circ s_{M}$ is the inclusion of $M$ into $C$. Let $N_{M}$ be a bounded submodule of $B$ containing the image of $s_{M}$. Then there is a bounded $V$-module map $t_{M}: N_{M} \rightarrow A$ such that $p \circ t_{M}$ is the inclusion of $N_{M}$ into $B$. So $t_{M} \circ s_{M}$ is the required local section for the map $A \rightarrow C$. It remains to to verify the pushout and pullback axioms. By 12 Lemma 2.3.1], the pullback of a bornological quotient map $A \stackrel{p}{\rightarrow} B$ along an arbitrary bounded $V$-module map $C \stackrel{f}{\rightarrow} B$ exists, and is a bornological quotient map $A \times_{p, f} C \stackrel{p^{\prime}}{\rightarrow} C$, where $A \times_{p, f} C=\{(x, y) \in A \oplus C: p(x)=f(y)\}$ and $p^{\prime}$ is the canonical projection onto $C$. This has an obvious local section: let $M \subseteq C$ be a bounded $V$-submodule. Then there exists a bounded $V$-module map $s_{f(M)}: f(M) \rightarrow A$ that locally splits $p$. So $\left(s_{f(M)} \circ f, \iota_{M}\right): M \rightarrow A \oplus E, m \mapsto\left(s_{f(M)} f(m), m\right)$, is the required local section of $p^{\prime}$.

Finally, let $i: A \nrightarrow B$ be an inflation, and $f: A \rightarrow A^{\prime}$ an arbitrary bounded $V$ module map. Then by Lemma $\mathbf{1 2}$ Lemma 2.3.1], the pushout exists, and is given by $B^{\prime}:=\frac{A^{\prime} \oplus B}{\{(f(a),-i(a)): a \in A\}}$ with the canonical maps $i^{\prime}: A^{\prime} \rightarrow B^{\prime}, i^{\prime}\left(a^{\prime}\right)=\left[\left(a^{\prime}, 0\right)\right]$ and $\hat{f}: B \rightarrow B^{\prime}, \hat{f}(b)=[(0, b)]$. Furthermore, $i^{\prime}$ is a bornological embedding. It remains to show that the cokernel $B^{\prime} \stackrel{q^{\prime}}{\rightarrow} \operatorname{coker}\left(i^{\prime}\right)$ is locally split. Since $i$ is an inflation, we know that for every bounded $V$-submodule $M$ of $\operatorname{coker}(i)$, there is a local section $s_{M}: M \rightarrow B$ for $B \rightarrow \operatorname{coker}(i)$. Then $\hat{f} \circ s$ is a local splitting of $q^{\prime}$.

Proposition 3.1.6. The pair $(\overleftarrow{\mathfrak{B}}, \overleftarrow{\mathfrak{E}})$ is an exact category

Proof. Let $p: A \rightarrow B$ and $q: B \rightarrow C$ be two deflations. By definition, we can represent $p$ and $q$ as diagrams of locally split cokernels $\left(p_{n}^{\prime}: A_{n}^{\prime} \rightarrow B_{n}^{\prime}\right)_{n \in \mathbb{N}}$ and $\left(q_{n}^{\prime \prime}: B_{n}^{\prime \prime} \rightarrow C_{n}^{\prime \prime}\right)_{n \in \mathbb{N}}$, where $A \cong\left(A_{n}^{\prime}\right)_{n \in \mathbb{N}},\left(B_{n}^{\prime}\right)_{n \in \mathbb{N}} \cong B \cong\left(B_{n}^{\prime \prime}\right)_{n \in \mathbb{N}}$ and $C \cong\left(C_{n}^{\prime \prime}\right)_{n \in \mathbb{N}}$. Taking the pullback of the projective system of cokernels $\left(p_{n}^{\prime}: A_{n}^{\prime} \rightarrow B_{n}^{\prime}\right)_{n \in \mathbb{N}}$ along the isomorphism $\left(B_{n}^{\prime \prime}\right)_{n \in \mathbb{N}} \rightarrow\left(B_{n}^{\prime}\right)_{n \in \mathbb{N}}$, we obtain a diagram of cokernels $p^{\prime \prime}:\left(A_{n}^{\prime \prime}\right)_{n \in \mathbb{N}} \rightarrow$ $\left(B_{n}^{\prime \prime}\right)_{n \in \mathbb{N}}$ such that $\left(A_{n}^{\prime \prime}\right)_{n \in \mathbb{N}} \cong\left(A_{n}^{\prime}\right)_{n \in \mathbb{N}} \cong A$. It is easy to see that $p^{\prime \prime}$ is locally split, so that it represents the deflation $p$. So for each $n \in \mathbb{N}$, there exists $k \geq n$, such that the structure map $B_{k}^{\prime \prime} \rightarrow B_{n}^{\prime \prime}$ is locally liftable with respect to $p_{n}^{\prime \prime}: A_{n}^{\prime \prime} \rightarrow B_{n}^{\prime \prime}$. Similarly, there exists $l \geq k$ such that the structure map $C_{l}^{\prime \prime} \rightarrow C_{k}^{\prime \prime}$ is locally liftable with respect to $q_{k}^{\prime \prime}: B_{k}^{\prime \prime} \rightarrow C_{k}^{\prime \prime}$. Composition of these local liftings $C_{l}^{\prime \prime} \rightarrow B_{k}^{\prime \prime} \rightarrow A_{n}^{\prime \prime}$ yields the required local lifting of the structure map $C_{l}^{\prime \prime} \rightarrow C_{n}^{\prime \prime}$ into the quotient $A_{n}^{\prime \prime} \rightarrow C_{n}^{\prime \prime}$.

To verify the other axioms, it is well known (see [30 Proposition 7.1.5]) that if the pullback (respectively, pushout) of a cokernel (respectively, kernel) along an arbitrary morphism exists and is a cokernel (respectively, a kernel), then the same holds in the projective category. We sketch the proof here as follows: let $q$ : $B \rightarrow C$ 
be a cokernel of projective systems and let $f: X \rightarrow C$ be an arbitrary pro-linear map. Then $q$ can be represented as a cokernel of diagrams $\left(q_{n}: B_{n} \rightarrow C_{n}\right)_{n \in \mathbb{N}}$. Modifying the terms $B_{n}$ and $C_{n}$ if necessary (up to an isomorphism of projective systems), we get a morphism of diagrams $f_{n}: X_{n} \rightarrow C_{n}$ into $B_{n} \rightarrow C_{n}$. Of course, the modified morphism of diagrams $B_{n} \rightarrow C_{n}$ still represents the deflation $q: B \rightarrow C$, so we continue to denote it by $q_{n}$. By Lemma 3.1.5, we can take the pullback of $q_{n}: B_{n} \rightarrow C_{n}$ along $f_{n}: X_{n} \rightarrow C_{n}$ and again obtain a cokernel $\left(B_{n} \times_{q, f} X_{n}\right) \rightarrow\left(X_{n}\right)$ of diagrams. Fix $n \in \mathbb{N}$. Since $q$ is a deflation, there is an $m \geq n$ such that the structure map $C_{m} \rightarrow C_{n}$ lifts locally with respect to the cokernel $B_{n} \rightarrow C_{n}$. Precomposing with $f_{m}: X_{m} \rightarrow C_{m}$ yields the required local lifting $X_{m} \rightarrow B_{n} \times_{q, f} X_{n}$ of the structure map $X_{m} \rightarrow X_{n}$.

The pushout axiom can be dealt with similarly: let $i: A \nrightarrow B$ be an inflation and $f: A \rightarrow A^{\prime}$ a pro-linear map. By definition, we can represent the extension $i: A \nrightarrow$ $B \stackrel{q}{\rightarrow} \operatorname{coker}(i)$ as a diagram of extensions $\left(A_{n} \stackrel{i_{n}}{\rightarrow} B_{n} \rightarrow \operatorname{coker}\left(i_{n}\right)\right)_{n \in \mathbb{N}}$ that satisfies: for each $n \in \mathbb{N}$, there is an $m \geq n$ such that the structure map $\operatorname{coker}\left(i_{m}\right) \rightarrow \operatorname{coker}\left(i_{n}\right)$ is locally liftable. By modifying $A$ and $B$ up to an isomorphism of projective systems, we can represent $f$ as a morphism of diagrams $f_{n}: A_{n} \rightarrow A_{n}^{\prime}$ out of the kernels $\left(i_{n}: A_{n} \rightarrow B_{n}\right)_{n \in \mathbb{N}}$. We continue to denote the kernel $A_{n} \rightarrow B_{n}$ by $i_{n}$ as both these morphisms represent $i: A \nrightarrow B$. By Lemma 3.1.5, we can take the pushout $A_{n}^{\prime} \stackrel{i_{n}^{\prime}}{\rightarrow} B_{n}^{\prime}$, whose cokernel isomorphic as a projective system to coker $(i)$

3.2. Definition of the derived category. We define the derived category of the exact category $(\overleftarrow{\mathfrak{B}}, \overleftarrow{\mathfrak{E}})$. Throughout this section, we will refer to $\mathbb{Z}_{2}$-graded chain complexes, also known as supercomplexes.

LEMMA 3.2.1. Let $R$ be a ring that is finitely generated as a $\mathbb{Z}$-module. Let $\left(X, \sigma_{X}\right)$ and $\left(Y, \sigma_{Y}\right)$ be projective systems of $R$-modules, and $f: X \rightarrow Y$ a morphism of projective system of $\mathbb{Z}$-modules that is $R$-linear in the sense that

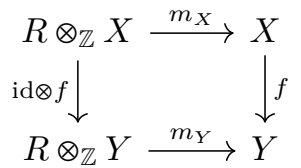

commutes, where $m_{X}$ and $m_{Y}$ are the multiplication maps of $X$ and $Y$, respectively. Then $f$ can be represented as a morphism of projective system of $R$-modules.

Proof. We can represent $f$ by $\mathbb{Z}$-linear maps $\left(f_{n}: X_{m(n)} \rightarrow Y_{n}\right)_{n \in \mathbb{N}}$. The condition of $R$-linearity says that for each generator $r \in R$, there are indices $k_{r}(n) \geq$ $l_{r}(n) \geq m(n)$ to have $f_{n}\left(\sigma^{X}(r \cdot x)\right)=r \cdot \sigma^{Y}\left(f_{l_{r}}(x)\right)$ for $x \in X_{k_{r}(n)}$. Now since $R$ is finitely generated, we can arrange that this equality holds simultaneously on all the generators of $R$, by taking the maximum of all such indices $k_{r}$. So $f: X \rightarrow Y$ is a morphism of projective systems of $R$-modules.

Theorem 3.2.2. Let $\mathcal{C}$ be an additive category with cokernels. Then any supercomplex in $\overleftarrow{\mathcal{C}}$ is a projective limit of a projective system of supercomplexes in $\mathcal{C}$ and, this yields an equivalence of categories

$$
\overleftarrow{\operatorname{Kom}(\mathcal{C})} \cong \operatorname{Kom}(\overleftarrow{\mathcal{C}})
$$


Proof. Let $R$ be the ring with the presentation $\left\{g, d: g^{2}=1, g d+d c=0, d^{2}=0\right\}$. Then a $\mathbb{Z}_{2}$-graded chain complex over $\mathcal{C}$ is equivalent to an object $X \in \mathcal{C}$, together with a ring homomorphism $R \rightarrow \operatorname{End}(X)$. Since $\mathcal{C}$ is additive and has cokernels, so does $\overleftarrow{\mathcal{C}}$. Therefore any chain complex in $\operatorname{Kom}(\overleftarrow{\mathcal{C}})$ is a projective system $X=\left(X_{n}\right)_{n \in N}$ in $\overleftarrow{\mathcal{C}}$, together with a ring homomorphism $f: R \rightarrow \operatorname{End}(X)$. Viewing $R$ as an $R$-module in an obvious way, we obtain a projective system $R \otimes_{\mathbb{Z}} X$ of $R$-modules. Since $R$ is finitely generated and free as an abelian group and, since $\overleftarrow{\mathcal{C}}$ is an additive category, $R \otimes_{\mathbb{Z}} X$ is well-defined. It is concretely given by a direct sum of finitely many copies of $X$. The map $f$ induces a morphism $R \otimes_{\mathbb{Z}} X \rightarrow X$ of projective systems in $\overleftarrow{\mathcal{C}}$ Tensoring on the left with $R$, we obtain a morphism $R \otimes_{\mathbb{Z}} R \otimes_{\mathbb{Z}} X \rightarrow R \otimes_{\mathbb{Z}} X$ of projective systems in $\overleftarrow{\mathcal{C}}$, which is $R$-linear. Since $R$ is finitely generated, Lemma 3.2.1 implies that we can represent this map as a projective system of $R$-module maps $R \otimes_{\mathbb{Z}} R \otimes_{\mathbb{Z}} X \rightarrow R \otimes_{\mathbb{Z}} X$. These can be further represented as a diagram of $R$-modules $\left(R \otimes_{\mathbb{Z}} R \otimes_{\mathbb{Z}} X_{n} \rightarrow R \otimes_{\mathbb{Z}} X_{n}\right)_{n \in M}$, after suitably reindexing by some directed set $M$, with $\left(X_{n}\right)_{n \in M} \cong X$ in $\overleftarrow{\mathcal{C}}$. For each $n$, the cokernel of $R \otimes_{\mathbb{Z}} R \otimes_{\mathbb{Z}} X_{n} \rightarrow R \otimes_{\mathbb{Z}} X_{n}$ is $X_{n}$, so that each $X_{n}$ is an $R$-module. Therefore, $X$ is a projective system of $R$-modules. By naturality of the bar resolution, this assignment is indeed a functor $\operatorname{Kom}(\overleftarrow{\mathcal{C}}) \rightarrow \overleftarrow{\operatorname{Kom}(\mathcal{C})}$, which is inverse to the functor $\overleftarrow{\operatorname{Kom}(\mathcal{C})} \rightarrow \operatorname{Kom}(\overleftarrow{\mathcal{C}})$ that forgets the $R$-action on a diagram in $\mathcal{C}$.

Definition 3.2.3. A chain complex $(C, \delta)$ in an exact category is called exact if $\operatorname{ker}(\delta)$ exists and

$$
\operatorname{ker}(\delta) \nrightarrow C \rightarrow \operatorname{ker}(\delta)
$$

is a conflation, where $\operatorname{ker}(\delta) \rightarrow C$ is the identical inclusion and $C \rightarrow \operatorname{ker}(\delta)$ is the map $\delta$ at each level of the chain complex. A chain map $f: C \rightarrow D$ is called a quasi-isomorphism if its mapping cone is exact.

Consider the exact category $(\overleftarrow{\mathfrak{B}}, \overleftarrow{\mathfrak{E}})$ of projective systems of torsion-free bornological $V$-modules with locally split extensions as conflations as in Definition 3.1.3. Then $\operatorname{Kom}(\overleftarrow{\mathfrak{B}})$ is an exact category, whose conflations are given degreewise. We use the equivalence of categories $\overleftarrow{\operatorname{Kom}(\mathfrak{B})} \cong \operatorname{Kom}(\overleftarrow{\mathfrak{B}})$ to describe conflations in the category $\overleftarrow{\operatorname{Kom}(\mathfrak{B})}$, which is easier to work with. The induced exact category structure on $\overleftarrow{\operatorname{Kom}(\mathfrak{B})}$ is the set of all kernel-cokernel pairs that are mapped by the forgetful functor to conflations in $\operatorname{Kom}(\overleftarrow{\mathfrak{B}})$. By $[\mathbf{8}$, Section 5], this is indeed an exact structure on $\overleftarrow{\operatorname{Kom}(\mathfrak{B})}$. We describe these conflations more explicitly.

Let $K \nrightarrow E \rightarrow Q$ be a locally split extension of chain complexes in each degree. This means that at each chain complex degree, this extension is isomorphic to a projective system of extensions of bornological $V$-modules

$$
\left(K_{n} \nrightarrow E_{n} \rightarrow Q_{n}\right)_{n \in \mathbb{N}}
$$

that is locally split. Since the forgetful functor $\overleftarrow{\operatorname{Kom}(\mathfrak{B})} \rightarrow \operatorname{Kom}(\overleftarrow{\mathfrak{B}})$ is part of an equivalence of categories, it is essentially surjective and fully faithful. So the extension above lifts to the extension of projective systems of chain complexes

$$
\left(K^{\prime}, d^{K^{\prime}}\right) \nrightarrow\left(E^{\prime}, d^{E^{\prime}}\right) \rightarrow\left(Q^{\prime}, d^{Q^{\prime}}\right)
$$


that is isomorphic to the original extension and satisfies the following: for each $n \in \mathbb{N}$, there exists $m \geq n$ such that the canonical chain map $Q_{m}^{\prime} \rightarrow Q_{n}^{\prime}$ is locally liftable with respect to the extension $K_{n}^{\prime} \gg E_{n}^{\prime} \rightarrow Q_{n}^{\prime}$ taken degreewise.

In what follows, we also refer to conflations in $\overleftarrow{\operatorname{Kom}(\mathfrak{B})}$ as locally split extensions and not explicitly distinguish between conflations in $\overleftarrow{\operatorname{Kom}(\mathfrak{B})}$ and $\operatorname{Kom}(\overleftarrow{\mathfrak{B}})$

Definition 3.2.4. The derived category of the exact category $\overleftarrow{\mathfrak{B}}$ is the localisation of the homotopy category of chain complexes $\operatorname{HoKom}(\overleftarrow{\mathfrak{B}})$ at the quasiisomorphisms.

3.3. Locally contractible chain complexes and local chain homotopy equivalences. In this section, we describe quasi-isomorphisms and exact chain complexes as local chain homotopy equivalences and locally contractible complexes. This description will be useful in the next section, when we compare the analytic cyclic homology of an $\mathbb{F}$-algebra $A$ and the analytic cyclic homology of a torsion-free lifting. We will make use of the local structure of complete, torsion-free bornological $V$-modules to construct an explicit local chain homotopy equivalence between them.

Let $C=\left(C_{n}, d_{n}^{C}\right)$ be a chain complex of bornological $V$-modules. For each $n$, we write the bornological $V$-module $C_{n}$ as an inductive limit of its bounded submodules as follows $C_{n}=\lim _{i \in I_{n}} C_{n}^{i}$.

Definition 3.3.1. (1) A local chain map $f:\left(C, d^{C}\right) \rightarrow\left(D, d^{D}\right)$ between chain complexes in $\operatorname{Kom}(\mathfrak{B})$ is a collection of $V$-linear maps $\left(f_{n}^{i}: C_{n}^{i} \rightarrow\right.$ $\left.D_{n}\right)_{\left(n \in \mathbb{Z}_{2}, i \in I_{n}\right)}$ satisfying $d_{n}^{D} \circ f_{n}^{i}(x)=f_{n-1}^{j} \circ d_{n}^{C}(x)$ for all $x \in C_{n}^{i}$, and some $j \in I_{n-1}$ so that $d_{n}^{C}\left(C_{n}^{i}\right) \subseteq C_{n-1}^{j}$.

EXAMPLE 3.3.2. The collection of canonical maps $\left(C_{n}^{i} \hookrightarrow C_{n}\right)_{\left(n \in \mathbb{Z}, i \in I_{n}\right)}$ is a local chain map $C \rightarrow C$.

(2) Two local chain maps $f, g:\left(C, d^{C}\right) \rightarrow\left(D, d^{D}\right)$ in $\operatorname{Kom}(\mathfrak{B})$ are said to be locally chain homotopic if there is a collection of bounded $V$-module maps

$$
\left.\left(h_{n}^{i_{n}}: C_{n}^{i_{n}}+d_{n}^{C}\left(C_{n}^{i_{n}}\right) \rightarrow D_{n+1}\right)_{\left(n \in \mathbb{Z}_{2}, i_{n} \in I_{n}\right.}\right)
$$

that satisfies

$$
f_{n}^{k}(x)-g_{n}^{k}(x)=\left(h_{n-1}^{i_{n}} \circ d_{n}^{C}+d_{n+1}^{D} \circ h_{n}^{i_{n}}\right)(x)
$$

for all $x \in C_{n}^{i_{n}}+d_{n}^{C}\left(C_{n}^{i_{n}}\right)$ and all $\left(n, i_{n}\right) \in\left(\mathbb{Z}_{2}, I_{n}\right)$. We call the collection above a local chain homotopy and denote it by $h: C \rightarrow D[1]$.

REMARK 3.3.3. Before moving on, we simplify our notation. Since we rarely need the chain complex degree $n$, we omit it altogether. A bounded submodule at each chain complex degree will simply be denoted as $M \subseteq C$. Accordingly, a local chain homotopy is a collection of bounded $V$-module maps $h: M+d^{C}(M) \rightarrow D$ that satisfies $f(x)-g(x)=\left(h \circ d^{C}+d^{D} \circ h\right)(x)$ for all $x \in M+d^{C}(M)$ and every bounded submodule $M$.

Definition 3.3.4. A chain complex $\left(C, d^{C}\right)$ in $\operatorname{Kom}(\mathfrak{B})$ is locally contractible if for any bounded submodule $M \subseteq C$, there is a bounded $V$-module map $h^{M}: M+$ $d^{C}(M) \rightarrow C$ such that $\left(h^{M} \circ d^{C}+d^{D} \circ h^{M}\right)(x)=x$ for all $x \in M+d^{C}(M)$. In other words, the identical inclusions of each bounded subcomplex of $C$ is null homotopic. 
We now define local contractibility and local chain homotopy equivalences for projective systems of chain complexes and chain maps in $\overleftarrow{\operatorname{Kom}(\mathfrak{B})}$.

Definition 3.3.5. A projective system of chain complexes $C=\left(C_{n}, \gamma_{m, n}: C_{m} \rightarrow\right.$ $\left.C_{n}\right)_{n \in N}$ in $\overleftarrow{\operatorname{Kom}(\mathfrak{B})}$ is locally contractible if and only if for every $n$, there are an $m \geq n$ such that the structure map $\gamma_{m, n}: C_{m(n)} \rightarrow C_{n}$ is locally null-homotopic.

Definition 3.3.6. A projective system of chain maps $f: C \rightarrow D$ in $\overleftarrow{\operatorname{Kom}(\mathfrak{B})}$ represented by a projective system of chain maps $\left(f_{n}: C_{n} \rightarrow D_{n}\right)_{n \in \mathbb{N}}$ is called a local chain homotopy equivalence if for each $n \in \mathbb{N}$, there are an $m \geq n$, and a local chain map and local chain homotopies

$$
g_{m}: D_{m} \rightarrow C_{n}, \quad h: D_{m} \rightarrow D_{n}[1], \quad h: C_{m} \rightarrow C_{n}[1]
$$

between $f_{n} g_{m}$ and $g_{m} f_{m}$, and the canonical structure maps $D_{m} \rightarrow D_{n}$ and $C_{m} \rightarrow C_{n}$, respectively.

Lemma 3.3.7. A projective system $f: C \rightarrow D$ of chain maps $\overleftarrow{\mathrm{Kom}(\mathfrak{B})}$ is a local chain homotopy equivalence if and only if cone $(f)$ is locally contractible. A projective system of chain complexes $C$ is locally contractible if and only if the zero map $0 \rightarrow C$ is a local chain homotopy equivalence.

Proof. Let cone $(f)=C[-1] \oplus D$ be a locally contractible complex in $\overleftarrow{\operatorname{Kom}(\mathfrak{B})}$ Then for each $n$, there exists $m(n) \geq n$ such that the structure map

$$
\operatorname{cone}(f)_{m}=C[-1]_{m} \oplus D_{m} \stackrel{\gamma_{m, n}^{C[-1]} \oplus \gamma_{m, n}^{D}}{\longrightarrow} C[-1]_{n} \oplus D_{n}=\operatorname{cone}(f)_{n}
$$

is locally null homotopic. So for every bounded subset $M \subseteq \operatorname{cone}(f)_{m}$, there exists a bounded contracting homotopy $h: M+d^{\operatorname{cone}(f)}(M) \rightarrow \operatorname{cone}(f)_{n}$. Since cone $(f)_{m}$ has the direct sum bornology, $M$ is contained in $T_{C} \oplus S_{D}$, with bounded subsets $T_{C} \subseteq C[-1]$ and $S_{D} \subseteq D$. More concretely, these bounded subsets are of the form $T_{C}=M^{C[-1]}+d^{C[-1]}\left(M^{C[-1]}\right)$ and $S_{D}=M^{D}+f_{m}\left(M^{C[-1]}\right)+d^{D}\left(M^{D}\right)$, for bounded subsets $M^{C[-1]} \subseteq C[-1]_{m}$ and $M^{D} \subseteq D_{m}$. So the local homotopy $h: T_{C} \oplus S_{D} \rightarrow \operatorname{cone}(f)_{n}$ is given by a block matrix of the form

$$
\left(\begin{array}{ll}
h_{11} & h_{12} \\
h_{21} & h_{22}
\end{array}\right): T_{C} \oplus S_{D} \rightarrow C[-1]_{n} \oplus D_{n} .
$$

For two linear maps, denote $[f, g]:=f g+g f$. Then the local null homotopy of the structure map cone $(f)_{m} \rightarrow \operatorname{cone}(f)_{n}$ says that

$$
\begin{aligned}
& \left(\begin{array}{cc}
\gamma_{m, n}^{C[-1]} & 0 \\
0 & \gamma_{m, n}^{D}
\end{array}\right) \\
& \quad=\left(\begin{array}{cc}
{\left[d^{C[-1]}, h_{11}\right]+h_{12} \circ f} & d^{C[-1]} \circ h_{12}-h_{12} d^{D} \\
h_{21} \circ d^{C[-1]}-d^{D} \circ h_{21}+h_{22} \circ f+f \circ h_{11} & f \circ h_{12}-\left[d^{D}, h_{22}\right]
\end{array}\right)
\end{aligned}
$$

on the bounded submodule $T_{C} \oplus S_{D}$. Then $g_{m}=h_{12}: S_{D} \rightarrow D_{n}$ is the required local chain homotopy inverse of $f_{n}: C_{n} \rightarrow D_{n}$.

Conversely, if $f$ is a local chain homotopy equivalence, then the local chain homotopy inverse and the local chain homotopies can be used to satisfy (3.3.8), which shows that cone $(f)$ is locally contractible. 
Theorem 3.3.9. A chain complex $C$ in $\overleftarrow{\operatorname{Kom}(\mathfrak{B})} \cong \operatorname{Kom}(\overleftarrow{\mathfrak{B}})$ is locally contractible if and only if it is locally split exact. A chain map $f: C \rightarrow D$ in $\overleftarrow{\operatorname{Kom}(\mathfrak{B})}$ is a local chain homotopy equivalence if and only if it is a quasi-isomorphism.

Proof. Let $C=\left(C_{n}, d_{n}\right)_{n \in N}$ be a locally contractible projective system of chain complexes. Then for each $n \in \mathbb{N}$, there exists $m \geq n$ such that the structure map $C_{m} \stackrel{\gamma_{m, n}}{\longrightarrow} C_{n}$ is locally null-homotopic. So the restriction of $\gamma_{m, n}$ to $\operatorname{ker}\left(d_{m}\right) \subseteq C_{m}$ is locally null-homotopic. So for any bounded submodule $X \subseteq \operatorname{ker}\left(d_{m}\right)$, there exists a bounded $V$-module map $h: X \rightarrow C_{n}$ witnessing the null-homotopy, that is, $d_{n} \circ h=\gamma_{n, m} \circ \iota_{X}$. This means precisely that the canonical map $\operatorname{ker}\left(d_{m}\right) \rightarrow \operatorname{ker}\left(d_{n}\right)$ is locally liftable with respect to the map $C_{n} \rightarrow \operatorname{ker}\left(d_{n}\right)$. Finally, since $C$ has zero homology as a projective system, the map $d: C \rightarrow \operatorname{ker}(d)$ is surjective as a morphism of projective systems. Therefore, we can arrange that $\operatorname{ker}(d) \nrightarrow C \rightarrow \operatorname{ker}(d)$ is isomorphic to a projective system of extensions in $\overleftarrow{\mathfrak{B}}$ that is locally split exact. So $C$ is locally split exact.

Conversely, suppose $C$ is locally split exact. Then $\left(\operatorname{im}\left(d_{n}\right)\right)_{n \in \mathbb{N}} \cong\left(\operatorname{ker}\left(d_{n}\right)\right)_{n \in \mathbb{N}}$ as projective systems. And local split exactness implies that for each $n$, there is an $m \geq n$, such that for every bounded submodule $X \subseteq \operatorname{ker}\left(d_{m}\right)$, there is a bounded $V$-module map $h_{1}: X \rightarrow C_{m}$ satisfying $d_{n} \circ h_{1}=\gamma_{n, m} \circ \iota_{X}$. Similarly, for this $m$, there is an $l \geq m$ and a bounded $V$-module map $h_{2}: d_{l}(Y) \rightarrow C_{m}$ satisfying $d_{m} \circ h_{2}=\gamma_{m, l} \circ \iota_{d_{l}(Y)}$ for any bounded submodule $Y \subseteq C_{l}$. Define $\varphi_{Y}:=\gamma_{l, m} \circ \iota_{Y}-h_{2} \circ d_{l}: Y \rightarrow C_{m}$. Then $\varphi_{Y}$ is a bounded $V$-module map for the subspace bornology on $Y \subseteq C_{l}$, whose image is containted in $\operatorname{ker}\left(d_{m}\right)$. By hypothesis, $h_{1}$ exists on this bounded submodule to yield a local contracting homotopy for the structure map $\gamma_{n, l}: C_{l} \rightarrow C_{n}$.

The claim for local homotopy equivalences follows from Lemma 3.3.7 and the definition of quasi-isomorphisms.

3.4. Homotopy inverse limit. Let $C=\left(C_{n}, \gamma_{m, n}\right)_{n \in \mathbb{N}} \in \overleftarrow{\operatorname{Kom}(\mathfrak{B})}$ be a projective system of chain complexes of torsion-free bornological $V$-modules. Then for each $n \in \mathbb{N}$, there is a chain map

$$
\prod_{k=0}^{n} C_{k} \stackrel{\text { id-Shift }}{\longrightarrow} \prod_{k=0}^{n-1} C_{k}
$$

given explicitly as

$$
\left(\text { id }-\operatorname{Shift}_{n}\right)\left(c_{0}, \ldots, c_{n}\right) \mapsto\left(c_{0}-\gamma_{0,1}\left(c_{1}\right), c_{1}-\gamma_{1,2}\left(c_{2}\right), \ldots, c_{n-1}-\gamma_{n-1, n}\left(c_{n}\right)\right)
$$

for $c_{k} \in C_{k}, k=0, \ldots, n$. Furthermore, we have a canonical inclusion $C_{n} \stackrel{i_{n}}{\rightarrow} \prod_{k=0}^{n} C_{k}$, $i_{n}\left(x_{n}\right)=\left(\gamma_{0, n}\left(x_{n}\right), \ldots, \gamma_{n-1, n}\left(x_{n}\right)\right)$ whose composition with id - Shift $_{n}$ vanishes.

Lemma 3.4.1. Let $C \in \overleftarrow{\operatorname{Kom}(\mathfrak{B})}$ be a projective system of chain complexes indexed by $\mathbb{N}$. Then we have a split exact sequence

$$
C_{n} \stackrel{i_{n}}{\rightarrow} \prod_{k=0}^{n} C_{k} \stackrel{\text { id-Shift }}{\longrightarrow} \prod_{k=0}^{n-1} C_{k}
$$

Proof. The splitting of $i$ is given by the obvious projection

$$
\prod_{k=0}^{n} C_{k} \rightarrow C_{n} \quad\left(x_{0}, \ldots, x_{n}\right) \mapsto x_{n} .
$$


Now use the splitting lemma.

Using the lemma above, we deduce that for each $n \in \mathbb{N}$, there is a chain homotopy equivalence

$$
C_{n} \rightarrow \operatorname{cone}\left(1-\operatorname{Shift}_{n}\right) .
$$

Denote by $R C$ the projective system of chain complexes (cone $\left.\left(1-\operatorname{Shift}_{n}\right)\right)_{n \in \mathbb{N}}$. We then have a local chain homotopy equivalence between projective systems of chain complexes

$$
\alpha: C \rightarrow R C .
$$

REMARK 3.4.2. We note that the assignment $C \mapsto R C$ is only functorial for diagrams $\mathbb{N}^{\text {op }} \rightarrow \operatorname{Kom}(\mathfrak{B})$. To show that it is functorial for projective systems of chain maps, we must pass to the derived category $\operatorname{Der}(\overleftarrow{\mathfrak{B}})$. Let $f: C \rightarrow D$ be a projective system of chain maps. Since $C \rightarrow R C$ and $D \rightarrow R D$ are both isomorphisms in the derived category $\operatorname{Der}(\overleftarrow{\mathfrak{B}})$, the chain map $f$ lifts to a morphism $R(f): R C \rightarrow R D$ in $\operatorname{Der}(\overleftarrow{\mathfrak{B}})$, which is unique up to chain homotopy. So defining the action of $R$ on morphisms this way turns it into a functor $\overleftarrow{\operatorname{Kom}(\mathfrak{B})} \rightarrow \operatorname{Der}(\overleftarrow{\mathfrak{B}})$. The functoriality of homotopy inverse limits on a quasi-abelian category of pro-objects over arbitrary indexing categories is treated in detail in [30, Definition 7.3.5].

Definition 3.4.3. A functor $\mathcal{F}$ from $\operatorname{Kom}(\overleftarrow{\mathfrak{B}})$ or $\operatorname{HoKom}(\overleftarrow{\mathfrak{B}})$ to another category is called local if it maps local chain homotopy equivalences to isomorphisms.

To see how such functors can arise, let $F: \overleftarrow{\mathfrak{B}} \rightarrow \mathfrak{X}$ be an additive, exact functor for the exact category structure on $\overleftarrow{\mathfrak{B}}$ given by locally split extensions. Here $\mathfrak{X}$ is any exact category. Then $F$ induces a functor $F_{\star}: \operatorname{Kom}(\overleftarrow{\mathfrak{B}}) \rightarrow \operatorname{Kom}(\mathfrak{X})$ that descends to a functor $\operatorname{HoKom}(\overleftarrow{\mathfrak{B}}) \rightarrow \operatorname{Der}(\mathfrak{X})$. By Theorem 3.3.9 the functor $F$ is exact if and only if the functor $F_{\star}: \operatorname{HoKom}(\overleftarrow{\mathfrak{B}}) \rightarrow \operatorname{Der}(\mathfrak{X})$ is local.

A local functor extends to a functor on the derived category $\operatorname{Der}(\overleftarrow{\mathfrak{B}})$, by the universal property of localisations. We show that the functor $\lim _{\longleftarrow} \circ: \overleftarrow{\operatorname{Kom}(\mathfrak{B})} \rightarrow$ $\operatorname{Der}(\mathfrak{B})$ is a local functor.

Proposition 3.4.4. The functor $\lim \circ R: \overleftarrow{\operatorname{Kom}(\mathfrak{B})} \rightarrow \operatorname{Der}(\mathfrak{B})$ maps local chain homotopy equivalences to isomorphisms. So it descends to a functor

$$
\text { holim }:=\lim _{\longleftarrow} \circ R: \operatorname{Der}(\overleftarrow{\mathfrak{B}}) \rightarrow \operatorname{Der}(\mathfrak{B})
$$

which is called the homotopy inverse limit. The functor holim is the total right derived functor of the inverse limit functor.

Proof. Let $f: C \rightarrow D$ be a local chain homotopy equivalence. By Lemma 3.3.7, the mapping cone cone $(f)$ is a locally contractible projective system of chain complexes. We claim that $\lim \circ R(f)$ is a local chain homotopy equivalence. There is a representative of $f$ of the form $\left(f_{n}: C_{n} \rightarrow D_{n}\right)_{n \in \mathbb{N}}$. By naturality of the mapping cone, we get cone $\left(\lim \circ R\left(f_{n}\right)\right) \cong \lim \circ R(\operatorname{cone}(f))$. Using Remark 3.4.2 we see that $\lim _{\longleftarrow} \circ R(\operatorname{cone}(f))$ is locally contractible, so that cone $\left(\lim _{\longleftarrow} \circ R\left(f_{n}\right)\right)$ is locally contractible. Hence $\lim \circ R(f)$ is a local chain homotopy equivalence.

Finally, to see that $\lim _{\longleftarrow} \circ R$ is the total right derived functor of $\lim _{\longleftarrow}$, one checks that if $F$ is any local functor on $\operatorname{HoKom}(\overleftarrow{\mathfrak{B}})$, then any natural transformation 
$F \Rightarrow \lim$ factors uniquely through $\lim _{\longleftarrow} \circ R$. This is a standard result in category theory (see 33 , Proposition 6.4.11] for details in a more general context).

REMARK 3.4.5. We end this section with a remark on the notion of homology that we need for our purposes. We define the homology of a chain complex $C \in \overleftarrow{\operatorname{Kom}(\mathfrak{B})}$ as the homology of the homotopy inverse limit of $C$. By Proposition 3.4.4 the homology functor

$$
H_{\star}: \overleftarrow{\operatorname{Kom}(\mathfrak{B})} \rightarrow \mathfrak{B}
$$

is already local.

3.5. Interaction of quasi-isomorphisms with quasi-completion. We now discuss the exactness of the quasi-completion functor introduced in Step 7 of Section 2.0 .5 To do this, we first describe the internal structure of torsion-free bornological $V$-modules.

Proposition 3.5.1. The category of torsion-free bornological $V$-modules $\mathfrak{B}$ is equivalent to the full subcategory of the category of strict inductive systems of torsion-free $V$-modules $\overrightarrow{\mathfrak{B}}$.

Proof. By [11, Proposition 2.5], the result is true if we do not restrict to torsion-free bornological $V$-modules. We work out the same proof in the subcategory of torsion-free $V$-modules. In one direction, we have the dissection functor

$$
\text { diss: } \mathfrak{B} \rightarrow \overrightarrow{\mathfrak{B}},
$$

which maps a torsion-free bornological $V$-module to the strict inductive system of its bounded submodules. Of course, these submodules are in particular torsion-free. The functor in the other direction is the inductive limit functor, that assigns to an inductive system $\left(M_{i}\right)_{i \in I}$ of torsion-free $V$-modules, its inductive limit $M=\lim _{\longrightarrow} M_{i}$ with the inductive limit bornology. This is a torsion-free bornological $V$-module.

The assignment $M \rightarrow \mathcal{Q}(M)$ is functorial: it is the composition of the dissection functor $M \mapsto\left(M_{i}\right)_{i \in I}$, the $\pi$-adic completion functor, and the inductive limit functor. Now suppose $A \nrightarrow B \rightarrow C$ is a locally split extension of torsion-free bornological $V$-modules. Then the exactness of each of the component functors of $\mathcal{Q}$ implies that we have an extension of torsion-free bornological $V$-modules

$$
\mathcal{Q}(A) \nrightarrow \mathcal{Q}(B) \rightarrow \mathcal{Q}(C) .
$$

Note that $\pi$-adic completion is indeed an exact functor since in particular, the quotient $C$ is torsion-free. Denoting by $\mathfrak{B}_{\text {triv }}$ the exact structure whose conflations are all extensions in $\mathfrak{B}$, what we have shown is the following:

Proposition 3.5.2. The quasi-completion functor $\mathcal{Q}: \mathfrak{B} \rightarrow \mathfrak{B}_{\text {triv }}$ is an exact functor.

The exactness of $\mathcal{Q}$ implies the following easy consequence:

Corollary 3.5.3. The functor $\mathcal{Q}$ induces a local functor

$$
\mathcal{Q}: \operatorname{HoKom}(\mathfrak{B}) \rightarrow \operatorname{Der}\left(\mathfrak{B}_{\text {triv }}\right) \text {. }
$$

That is, if $f: C \rightarrow D$ is a local chain homotopy equivalence, then $\mathcal{Q}(f): \mathcal{Q}(C) \rightarrow \mathcal{Q}(D)$ is a quasi-isomorphism. 
Proof. Since $\mathcal{Q}$ is an additive functor, it extends to the homotopy category of chain complexes over $\mathfrak{B}$, by termwise application. Now if $f: C \rightarrow D$ is a local chain homotopy equivalence, then its cone cone $(f)$ is locally split-exact by Theorem 3.3.9 By naturality of the mapping cone, we have cone $(\mathcal{Q}(f)) \cong \mathcal{Q}(\operatorname{cone}(f))$. By Proposition 3.5.2 $\mathcal{Q}($ cone $(f))$ is exact, and hence cone $(\mathcal{Q}(f))$ is exact. So $\mathcal{Q}(f)$ is a quasi-isomorphism.

\section{Independence of the choice of lifting}

In this section, we prove Proposition 2.1.8 which is our main result.

4.1. Some non-Archimedean analysis. The proof of Proposition 2.1.8 requires a lot of preparation for which we first recall some results on the structure of non-Archimedean Banach spaces.

Definition 4.1.1. Let $D$ be a set. Let $\mathrm{C}_{0}(D, V)$ be the set of all functions $f: D \rightarrow V$ such that for each $\delta>0$ there is a finite subset $F \subseteq D$ with $|f(x)|<\delta$ for all $x \in D \backslash F$. Define $\mathrm{C}_{0}(D, F)$ similarly. Equip both with the supremum norm.

ThEOREM 4.1.2. 12 Theorem 2.4.2] Let $W$ be a complete, bornologically torsion-free bornological $V$-module. Any $\pi$-adically complete bounded $V$-submodule $M$ of $W$ is isomorphic to $\mathrm{C}_{0}(D, V)$ for some set $D$.

LEMMA 4.1.3. Let $W$ be a bornologically torsion-free, complete bornological $V$-module. Assume that the quotient bornology on $W / \pi W$ is the fine one. Then the quotient bornology on $W / \pi^{m} W$ is the fine one for all $m \in \mathbb{N}$.

Proof. The claim is proven by induction on $m$, the case $m=0$ being trivial. Assume that the claim is true for $m$. Let $S \subseteq W$ be a bounded $V$-module. We must show that its image in $W / \pi^{m+1} W$ is finitely generated. By assumption, its image in $W / \pi W$ is finitely generated. So we may pick a finite set $x_{1}, \ldots, x_{n}$ with $S \subseteq \sum_{j=1}^{n} V x_{j}+\pi W$. Let $S_{1}:=\left(S+\sum_{j=1}^{n} V x_{j}\right) \cap \pi W$. Then $S_{1} \subseteq \pi W$ is bounded and $S \subseteq \sum_{j=1}^{n} V x_{j}+S_{1}$. Since $W$ is bornologically torsion-free, $\pi^{-1} S_{1}$ is bounded as well. And $S \subseteq \sum_{j=1}^{n} V x_{j}+\pi \cdot\left(\pi^{-1} S_{1}\right)$. The induction assumption applied to the image of $\pi^{-1} S_{1}$ in $W / \pi^{m} W$ gives finitely many elements $x_{n+1}, \ldots, x_{m}$ such that $\pi^{-1} S_{1} \subseteq \sum_{j=n+1}^{m} V x_{j}+\pi^{m} W$. Then $S \subseteq \sum_{j=1}^{m} V x_{j}+\pi^{m+1} W$ as desired.

LEMMA 4.1.4. Let $W_{1}, W_{2}$ be torsion-free, complete bornological $V$-modules. If the quotient bornologies on $W_{j} / \pi W_{j}$ for $j=1,2$ are fine, then the same is true for $W_{1} \otimes W_{2} / \pi W_{1} \otimes W_{2}$ and also for $\mathrm{T} W_{1} / \pi \cdot \mathrm{T} W_{1}$.

Proof. Any bounded subset of $W_{1} \otimes W_{2}$ is contained in the image of $M_{1} \otimes M_{2}$ for $\pi$-adically complete bounded $V$-submodules $M_{j} \subseteq W_{j}$ for $j=1,2$. By assumption, for $j=1,2$ there are finite subsets $S_{j} \subseteq W_{j}$ with $M_{j}=\sum_{x \in S_{j}} V \cdot x+\pi W_{j}$. Hence the image of $M_{1} \otimes M_{2}$ in $W_{1} \otimes W_{2}$ is contained in $\sum_{x \in S_{1}, y \in S_{2}} x \otimes y+\pi \cdot W_{1} \otimes W_{2}$. Then the quotient bornology on $W_{1} \otimes W_{2} / \pi W_{1} \otimes W_{2}$ is the fine one. By induction, it follows that the quotient bornology on $W_{1}^{\otimes n} / \pi W_{1}^{\otimes n}$ is the fine one for all $n \in \mathbb{N}$. And this is inherited by the direct sum $\mathrm{T} W_{1}=\bigoplus W_{1}^{\otimes n}$ because any bounded subset of $\mathrm{T} W_{1}$ is already contained in a finite subsum. 
4.2. Proof of Proposition 2.1.8. We now return to the proof of Proposition 2.1 .8 Recall that $W$ is a complete, bornologically torsion-free $V$-module with a $V$-module surjection $\varrho: W \rightarrow A$, that we assume is bounded in the fine bornology on $A$. Let $s: A \rightarrow W$ be a set-theoretic section of $\varrho$. The map $s$ induces a $V$-algebra homomorphism $s_{*}: V\langle A\rangle \rightarrow \mathrm{T} W$. The following lemma gives us the necessary control on bounded subsets of $\mathcal{U}\left(\mathrm{T} W, I^{l}\right)$ :

LEMMA 4.2.1. In the situation of Proposition 2.1.8, let $N$ be a bounded $V$-submodule of $\mathcal{U}\left(\mathrm{T} W, I^{l}\right)$.

- There are $a \in \mathbb{N}$, a finite subset $S \subseteq I$, and a bounded subset $B \subseteq \mathrm{T} W$ such that $N$ is contained in $\sum_{j=1}^{a} V \pi^{-j} S^{l j}+B$.

- There is a bounded subset $M \subseteq W$ such that $N$ is the image of a bounded subset in $\mathcal{U}\left(\mathrm{T} M,(\mathrm{~T} M \cap I)^{l}\right)$, and the natural bounded map from $\mathcal{U}(\mathrm{T} M,(\mathrm{~T} M \cap$ $\left.I)^{l}\right)$ to $\mathcal{U}\left(\mathrm{T} W, I^{l}\right)$ is injective.

- There is a bounded homomorphism $f_{M}: \mathcal{U}\left(\mathrm{T} M,(\mathrm{~T} M \cap I)^{l}\right) \rightarrow \mathcal{U}\left(V\langle A\rangle, J^{l}\right)$ with $p \circ f_{M}=\varrho^{\#}$.

- There is a bounded homomorphism

$$
H_{M}: \mathcal{U}\left(\mathrm{T} M,(\mathrm{~T} M \cap I)^{l}\right) \rightarrow \mathcal{U}\left(\mathrm{T} W, I^{l}\right) \otimes V[t]
$$

such that $\mathrm{ev}_{0} \circ H_{M}$ is the homomorphism $\mathcal{U}\left(\mathrm{T} M,(\mathrm{~T} M \cap I)^{l}\right) \hookrightarrow \mathcal{U}\left(\mathrm{T} W, I^{l}\right)$ induced by the inclusion map, $\mathrm{ev}_{1} \circ H_{M}=s_{*} \circ f_{M}$, and $\left(\varrho^{\#} \otimes \mathrm{id}_{V[t]}\right) \circ H_{M}$ is the constant homotopy $\varrho^{\#}$.

- If $N_{A} \subseteq V A$ is a bounded $V$-submodule with $s_{*}\left(N_{A}\right) \subseteq M$, then $s_{*}$ induces a bounded homomorphism $s_{*}: \mathcal{U}\left(\mathrm{T} N_{A},\left(J \cap \mathrm{T} N_{A}\right)^{l}\right) \rightarrow \mathcal{U}\left(\mathrm{T} M,(\mathrm{~T} M \cap I)^{l}\right)$. And there is a bounded homomorphism

$$
H_{V A}: \mathcal{U}\left(\mathrm{T} N_{A},\left(J \cap \mathrm{T} N_{A}\right)^{l}\right) \rightarrow \mathcal{U}\left(V\langle A\rangle, J^{l}\right) \otimes V[t]
$$

such that $\mathrm{ev}_{1} \circ H_{V A}=f_{M} \circ s_{*}$, the map $\mathrm{ev}_{0} \circ H_{V A}$ is equal to the inclusion $\operatorname{map} \mathcal{U}\left(\mathrm{T} N_{A},\left(J \cap \mathrm{T} N_{A}\right)^{l}\right) \hookrightarrow \mathcal{U}\left(V\langle A\rangle, J^{l}\right)$, and $\left(p \otimes \mathrm{id}_{V[t]}\right) \circ H_{V A}$ is the constant homotopy associated to $p: \mathcal{U}\left(\mathrm{T} N_{A},\left(J \cap \mathrm{T} N_{A}\right)^{l}\right) \rightarrow A$.

Proof. By construction, $\mathcal{U}\left(\mathrm{T} W, I^{l}\right)$ carries the subspace bornology from $\mathrm{T} W \otimes$ $F$. Hence $N$ is bounded in TW $\otimes F$. Then it is contained in $\pi^{-b} N^{\prime}$ for a bounded subset $N^{\prime} \subseteq \mathrm{T} W$. Lemmas 4.1.3 and 4.1.4 imply that the quotient bornology on $\mathrm{T} W / \pi^{b} \mathrm{~T} W$ is fine. Thus there are $x_{1}, \ldots, x_{n} \in N^{\prime}$ that generate the image of $N^{\prime}$ in $\mathrm{T} W / \pi^{b} \mathrm{~T} W$ as a $V$-module. Since $N^{\prime} \subseteq \mathcal{U}\left(\mathrm{T} W, I^{l}\right)=\sum_{j=0}^{\infty} \pi^{-j} I^{j l}$, each $x_{j} \in N^{\prime}$ may be written as a finite sum of products $\pi^{-1} y_{1} \cdots y_{l}$ with $y_{1}, \ldots, y_{l} \in I$, plus a term in TW. Let $S$ be the set of all factors $y_{m}$ that appear in these products. Then each element of $N$ may be written as a finite $V$-linear combination of elements of $\bigcup_{j=1}^{a} \pi^{-j} S^{j l}$, plus an element of $\mathrm{T} W$. Let $B:=\left(N+\sum_{j=1}^{a} V \pi^{-j} S^{j l}\right) \cap \mathrm{T} W$. This is bounded because TW is bornologically torsion-free, and $B$ and $S$ verify the first claim.

The subset $B \cup S$ of TW is bounded and hence contained in the image of $\oplus_{j=1}^{b} M^{\otimes n}$ for some $\pi$-adically complete, bounded $V$-submodule $M \subseteq W$ and some $b \in \mathbb{N}$. The inclusion $M \hookrightarrow W$ induces an injective bounded homomorphism $\mathrm{T} M \hookrightarrow$ TW and this remains so after tensoring with $F$. Hence we get an injective bounded homomorphism $\mathcal{U}\left(\mathrm{T} M,(I \cap \mathrm{T} M)^{l}\right) \hookrightarrow \mathcal{U}\left(\mathrm{T} W, I^{l}\right)$. By construction of $B$ and $S$, the subset $N$ is the image of a bounded subset of $\mathcal{U}\left(\mathrm{T} M,(I \cap \mathrm{T} M)^{l}\right)$. 
The image of the map $\left.\varrho\right|_{M}: M \rightarrow A$ has finite dimension. Let $a_{1}, \ldots, a_{j} \in A$ be a basis. Identify $M$ with $\mathrm{C}_{0}(D, V)$. For $i \in D$, let $\delta_{i} \in \mathrm{C}_{0}(D, V)$ be the characteristic function of $\{i\}$. Write $\varrho\left(\delta_{i}\right)$ as a linear combination of the basis elements $a_{1}, \ldots, a_{j}$ with coefficients in $\mathbb{F}$ and lift the coefficients to $V$ in any way. This defines an element of $V A$ supported in the finite set $\left\{a_{1}, \ldots, a_{j}\right\}$, which we call $f\left(\delta_{i}\right)$. Doing this for all $i \in D$, we get a unique $V$-linear map $M \rightarrow V A$ with the specified values on characteristic functions. It has finite rank and thus it is bounded for the fine bornology on $V A$. It is compatible with the projection to $A$. We lift further to a linear map $M \rightarrow \mathrm{T}(V A)=V\langle A\rangle$ in the canonical way and let $f_{M}$ be the induced homomorphism $\mathrm{T} M \rightarrow V\langle A\rangle$. Since this is compatible with the projections to $A$,

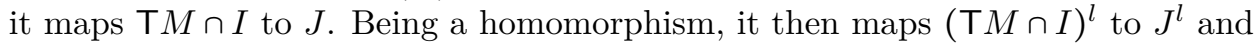
extends to a bounded homomorphism $f_{M}: \mathcal{U}\left(\mathrm{T} M,(\mathrm{~T} M \cap I)^{l}\right) \rightarrow \mathcal{U}\left(V\langle A\rangle, J^{l}\right)$.

To build $H_{M}$, we use the linear homotopy from the canonical inclusion $M \hookrightarrow W \hookrightarrow$ $\mathrm{T} W$ to the map $s_{*} \circ f_{M} \circ \sigma_{W}: M \hookrightarrow W \rightarrow \mathrm{T} W$. This bounded $V$-module map induces a bounded homomorphism $H_{M}: \mathrm{T} M \rightarrow \mathrm{T} W \otimes V[t]$. When we project to $A \otimes V[t]$, this map becomes the constant homotopy on $\left.\varrho^{\#}\right|_{\mathrm{T} M}$. Therefore, it maps the ideal $I \cap \mathrm{T} M$ into $I \otimes V[t]$. So its extension to $H_{M} \otimes \operatorname{id}_{F}: \mathrm{T} M \otimes F \rightarrow \mathrm{T} W \otimes V[t] \otimes F$ maps the tube algebra $\mathcal{U}\left(\mathrm{T} M,(\mathrm{~T} M \cap I)^{l}\right)$ into the tube algebra $\mathcal{U}\left(\mathrm{T} W \otimes V[t],(I \otimes V[t])^{l}\right)$. The latter is easily seen to be equal to $\mathcal{U}\left(T W, I^{l}\right) \otimes V[t]$. This provides the homotopy $H_{M}$ with the desired properties. The homotopy $H_{V A}$ is constructed in exactly the same way.

The statement of Lemma 4.2.1 is somewhat analogous to [12 Corollary 4.3.12], which says that all semi-split, analytically quasi-free, analytically nilpotent extensions of the same algebra are homotopy equivalent. Since the extensions we are dealing with are no longer semi-split, and then we only get locally defined homomorphisms.

The tube algebras $\left(\mathcal{U}\left(T W, I^{l}\right)\right)_{l \in \mathbb{N}^{*}}$ are only quasi-free as a pro-algebra, and not for each individual $l$. Therefore, we cannot apply the homotopy invariance of the $X$-complex to the local homomorphism in Lemma 4.2.1 directly. We could in principle describe a version of Lemma 4.2.1 which would say that the different tube algebra resolutions of $A$ are locally dagger homotopic as projective systems of bornological $V$-algebras. This would then have to be followed up with a version of homotopy invariance for the $X$-complex that works for locally dagger-homotopy equivalent algebras. Instead, we use the $B-b$-bicomplex $\mathbb{H} \mathbb{P}$, which is homotopy invariant even for individual tube algebras. Of course, the result will not change when we vary the tube algebra parameter $l$, since for quasi-free algebras, $\mathbb{H} \mathbb{P}$ is chain homotopy equivalent to the $X$-complex. The following lemma shows that the tube algebras that we require are indeed quasi-free.

Lemma 4.2.2. The pro-algebras $\mathcal{U}\left(V\langle A\rangle, J^{\infty}\right)_{\lg }$ and $\mathcal{U}\left(\mathrm{T} W, I^{\infty}\right)_{\lg }$ are quasi-free.

Proof. The proof is similar to the proof of [12 Proposition 4.4.6]. We only need to observe that the properties of being nilpotent $\bmod \pi$, and semi-dagger are both hereditary for extensions of pro-algebras, by [12 Proposition 4.2.5] and by adapting the proof of $\mathbf{1 2}$. Proposition 4.3.13].

Proof of Proposition 2.1.8. The map $f_{M}^{l}: \mathcal{U}\left(\mathrm{T} M,(\mathrm{~T} M \cap I)^{l}\right) \rightarrow \mathcal{U}\left(V\langle A\rangle, J^{l}\right)$ from Lemma 4.2 .1 is also a bounded algebra homomorphism

$$
f_{M}^{l}: \mathcal{U}\left(\mathrm{T} M,(\mathrm{~T} M \cap I)^{l}\right)_{\lg } \rightarrow \mathcal{U}\left(V\langle A\rangle, J^{l}\right)_{\lg }
$$


because application of the linear growth bornology is functorial. Furthermore, Lemma 4.2.1 implies that the bornological algebra $\mathcal{U}\left(\mathrm{T} W, I^{l}\right)_{\lg }$ is isomorphic to the inductive limit of the strict inductive system $\mathcal{U}\left(\mathrm{T} M,(\mathrm{~T} M \cap I)^{l}\right)_{\lg }$ over the directed set of bounded subsets $M \subseteq W$.

The polynomial homotopy $H_{M}: \mathcal{U}\left(\mathrm{T} M,(\mathrm{~T} M \cap I)^{l}\right) \rightarrow \mathcal{U}\left(\mathrm{T} W, I^{l}\right) \otimes V[t]$ between $s_{*} \circ f_{M}^{l}$ and the inclusion map $\mathcal{U}\left(\mathrm{T} M,(\mathrm{~T} M \cap I)^{l}\right) \stackrel{i_{M}}{\rightarrow} \mathcal{U}\left(\mathrm{T} W, I^{l}\right)$ is also a bounded algebra homomorphism

$$
H_{M}: \mathcal{U}\left(\mathrm{T} M,(\mathrm{~T} M \cap I)^{l}\right)_{\lg } \rightarrow \mathcal{U}\left(\mathrm{T} W, I^{l}\right)_{\lg } \otimes V[t]_{\lg }
$$

such that $\mathrm{ev}_{0} \circ H_{M}$ is the inclusion $\mathcal{U}\left(\mathrm{T} M,(\mathrm{~T} M \cap I)^{l}\right)_{\lg } \rightarrow \mathcal{U}\left(\mathrm{T} W, I^{m}\right)_{\lg }$ and $\mathrm{ev}_{1} \circ H_{M}$ is the composition $s_{*} \circ f_{M}^{l}$. Here we have used that the linear growth bornology commutes with tensor products (see 11. Proposition 3.1.25]). Similarly, the homomorphism $H_{V\langle A\rangle}$ is a polynomial homotopy between $f_{M}^{l} \circ s_{*}$ and the inclusion $\iota: \mathcal{U}\left(\mathrm{T} N_{A},\left(J \cap \mathrm{T} N_{A}\right)^{l}\right)_{\lg } \rightarrow \mathcal{U}\left(V\langle A\rangle, J^{l}\right)_{\lg }$. All this remains true after tensoring with $F$.

Fix $l \geq 1$. The bounded homomorphisms

$$
s_{*}^{l} \otimes F: \mathcal{U}\left(V\langle A\rangle, J^{l}\right)_{\lg } \otimes F \rightarrow \mathcal{U}\left(\mathrm{T} W, I^{l}\right)_{\lg } \otimes F
$$

and

$$
f_{M}^{l} \otimes F: \mathcal{U}\left(\mathrm{T} M,(\mathrm{~T} M \cap I)^{l}\right)_{\lg } \otimes F \rightarrow \mathcal{U}\left(V\langle A\rangle, J^{l}\right)_{\lg } \otimes F
$$

induce bounded chain maps between the bornological chain complexes

$$
\mathbb{H} \mathbb{P}\left(\mathcal{U}\left(V\langle A\rangle, J^{l}\right)_{\lg } \otimes F\right) \text { and } \mathbb{H} \mathbb{P}\left(\mathcal{U}\left(\mathrm{T} M,(\mathrm{~T} M \cap I)^{l}\right)_{\lg } \otimes F\right) .
$$

By homotopy invariance of periodic cyclic homology, the polynomial homotopies $H_{M}$ and $H_{V\langle A\rangle}$ induce chain homotopies between $\mathbb{H} \mathbb{P}\left(s_{*}^{l}\right) \circ \mathbb{H} \mathbb{P}\left(f_{M}^{l}\right)$ and $\mathbb{H} \mathbb{P}\left(i_{M}\right)$ and between $\mathbb{H} \mathbb{P}\left(f_{M}^{l}\right) \circ \mathbb{H} \mathbb{P}\left(s_{*}^{l}\right)$ and $\mathbb{H P}(i)$, respectively. Since $M$ is an arbitrary bounded submodule of $W$, we conclude that the pro-chain map

$$
\mathbb{H} \mathbb{P}\left(s_{*}^{l}\right): \mathbb{H} \mathbb{P}\left(\mathcal{U}\left(V\langle A\rangle, J^{l}\right)_{\lg } \otimes F\right) \rightarrow \mathbb{H} \mathbb{P}\left(\mathcal{U}\left(\mathrm{T} W, I^{l}\right)_{\lg } \otimes F\right)
$$

is a local chain homotopy equivalence.

By Lemma 4.2.2, the $b-B$-bicomplexes are homotopy equivalent to the $X$ complexes of these quasi-free pro- $F$-algebras. We then get a local chain homotopy equivalence

$$
\mathbb{H} \mathbb{A}(A)=X\left(\mathcal{U}\left(V\langle A\rangle, J^{\infty}\right)_{\lg } \otimes F\right) \rightarrow X\left(\mathcal{U}\left(\mathrm{T} W, I^{\infty}\right)_{\lg } \otimes F\right)=\mathbb{H} \mathbb{A}(A, W, \varrho)
$$

between projective systems of chain complexes of bornological $V$-modules. This is a quasi-isomorphism by Theorem 3.4.4 and Corollary 3.5.3

We end this section with a version of Proposition 2.1.8 for pro- $V$-module liftings. This version will be used to prove excision for our theory. Let $A$ be an $\mathbb{F}$-algebra viewed as a constant projective system. Let $W=\left(W_{n}\right)_{n \in \mathbb{N}}$ be a projective system of complete, bornologically torsion-free bornological $V$-modules with a compatible family of surjective $V$-module maps $\varrho_{n}: W_{n} \rightarrow A$ denoted by $\varrho: W \rightarrow A$. Let $s: A \rightarrow W$ be a bounded pro-set section. Assume that each $\varrho_{n}$ is bounded in the fine bornology on $A$.

Proposition 4.2.3. With $A, \varrho: W \rightarrow A$ and $s: A \rightarrow W$ as above, there is a canonical bornological $F$-module isomorphism

$$
\mathrm{HA}_{*}(A) \cong \mathrm{HA}_{*}(A ; W, \varrho) .
$$


Proof. By construction, there are maps $s_{n}: A \rightarrow W_{n}$ that split $\varrho_{n}: W_{n} \rightarrow A$ for each $n \in \mathbb{N}$. These maps induce $V$-algebra homomorphisms $V\langle A\rangle \rightarrow \mathrm{T} W_{n}$ for each $n$, which in turn induce pro-chain maps $\mathbb{H} \mathbb{A}(A) \rightarrow \mathbb{H} \mathbb{A}\left(A, W_{n}, \varrho_{n}\right)$. Since the local algebra homomorphisms of Lemma 4.2.1 are defined entrywise on each tensor algebra $\mathrm{T} W_{n}$, the pro-chain map above is a quasi-isomorphism for each $n$.

\section{Homotopy invariance and stability}

In this section, we prove certain formal properties of our theory. We start with a homotopy lifting result that is analogous to [12 Proposition 4.3.10]. Using this, we prove that our theory is homotopy invariant for polynomial homotopies. We then use the stability result $\mathbf{1 2}$, Proposition 6.2] of analytic cyclic homology for torsion-free algebras to show that the same result holds for stability over finitely supported matrices.

Lemma 5.0.1. Let $f_{0}, f_{1}: A \rightarrow B$ be homomorphisms between $\mathbb{F}$-algebras, and let $H: A \rightarrow B \otimes_{\mathbb{F}} \mathbb{F}[t]$ be a polynomial homotopy between them.

(1) there are $V$-algebra homomorphisms $f_{0}^{*}, f_{1}^{*}: V\langle A\rangle \rightarrow V\langle B\rangle$ lifting $f_{0}$ and $f_{1}$;

(2) there is a polynomial homotopy $H^{*}: V\langle A\rangle \rightarrow V\langle B\rangle \otimes V[t]$ between $f_{0}^{*}$ and $f_{1}^{*}$ that lifts $H$.

Proof. The liftings $f_{0}^{*}$ and $f_{1}^{*}$ exist because of the universal property of free algebras. The proof of the second statement is similar to the proof of $\mathbf{1 2}$, Proposition 4.3.10] and the universal property of free algebras. Concretely, we take the pull-back $\mathcal{E}$ of the evaluation maps $\left(\mathrm{ev}_{0}, \mathrm{ev}_{1}\right): B \otimes \mathbb{F}[t] \rightarrow B \oplus B$ along the canonical projection $V\langle B\rangle \oplus V\langle B\rangle \stackrel{p_{B} \oplus p_{B}}{\longrightarrow} B \oplus B$. Then the maps

$$
\rho: V\langle B\rangle \otimes V[t] \rightarrow V\langle B\rangle \oplus V\langle B\rangle, \quad b \mapsto(b(0), b(1)),
$$

and $V\langle B\rangle \otimes V[t] \stackrel{\eta}{\rightarrow} B \otimes_{\mathbb{F}} \mathbb{F}[t]$ induce a unique map $\phi: V\langle B\rangle \otimes V[t] \rightarrow \mathcal{E}$ by the universal property of pullbacks. Here $\eta=p_{B} \otimes \mathbb{F}$. We have the following commuting diagram:

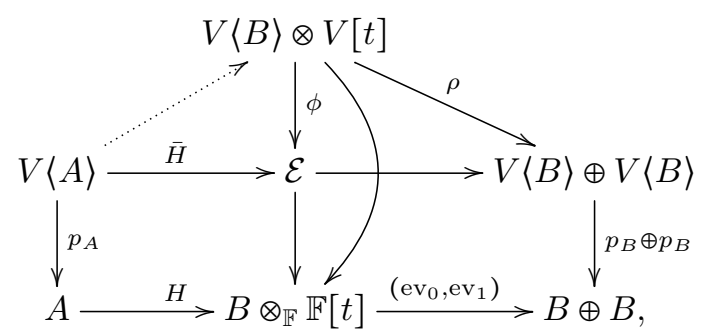

where $\bar{H}$ is defined on $A$ by $a \mapsto\left(H(a), f_{0}^{*}(a), f_{1}^{*}(a)\right)$. Composing with a section of $\phi$, we obtain a map of sets $A \rightarrow V\langle B\rangle \otimes V[t]$. By the universal property of free algebras, this extends to a $V$-algebra homomorphism $H^{*}: V\langle A\rangle \rightarrow V\langle B\rangle \otimes V[t]$, finishing the proof.

Proposition 5.0.2. Let $A$ be an $\mathbb{F}$-algebra and let $A[t]:=A \otimes_{\mathbb{F}} \mathbb{F}[t]$.

(1) the inclusion $\iota_{A}: A \rightarrow A \otimes_{\mathbb{F}} \mathbb{F}[t]$ induces a quasi-isomorphism $\mathbb{H} \mathbb{A}(A) \cong$ $\mathbb{H} \mathbb{A}(A[t])$; 
(2) let $\Lambda$ be a set and let $M_{\Lambda}(A)$ be the algebra of finitely supported matrices indexed by $\Lambda \times \Lambda$, with values in $A$. Then for each $\lambda \in \Lambda$, the canonical inclusion $\iota_{\lambda}: A \rightarrow M_{\Lambda}(A), a \mapsto e_{\lambda, \lambda} \otimes a$ induces a quasi-isomorphism

$$
\mathbb{H} \mathbb{A}(A) \cong \mathbb{H} \mathbb{A}\left(M_{\Lambda}(A)\right) .
$$

Proof. Let $\mathrm{ev}_{0}: A[t] \rightarrow A$ be the evaluation map at $t=0$. Then $H: A[t] \rightarrow$ $A[t, s], f \mapsto s f+(1-s)\left(\iota_{A} \circ \mathrm{ev}_{0}\right)(f)$ is an affine homotopy between $\iota \circ \mathrm{ev}_{0}$ and $\operatorname{id}_{A[t]}$. By Lemma 5.0.1. this extends to a polynomial homotopy $H^{*}: V\langle A[t]\rangle \rightarrow$ $V\langle A[t]\rangle \otimes V[s]$ between the induced maps $\iota^{*} \circ \mathrm{ev}_{0}^{*}$ and the identity map. Let $I_{V\langle A[t]\rangle}=\operatorname{ker}(V\langle A[t]\rangle \rightarrow A[t])$. Then $H^{*}$ maps $I_{V\langle A[t]\rangle}$ to $I_{V\langle A[t]\rangle} \otimes V[s]$. Hence it extends to an algebra homomorphism

$$
\mathcal{U}\left(V\langle A[t]\rangle, I_{V\langle A[t]\rangle}^{m}\right) \rightarrow \mathcal{U}\left(V\langle A\rangle, I_{V\langle A\rangle}^{m}\right) \otimes V[s],
$$

for each $m \geq 1$. This further induces bounded algebra homomorphisms

$$
\mathcal{U}\left(V\langle A[t]\rangle, I_{V\langle A[t]\rangle}^{m}\right)_{\lg } \rightarrow \mathcal{U}\left(V\langle A[t]\rangle, I_{V\langle A[t]\rangle}^{m}\right)_{\lg } \otimes V[s]_{\lg }
$$

for each $m \geq 1$, which are polynomial homotopies between the corresponding maps of semi-dagger algebras induced by $\iota^{*} \circ \mathrm{ev}_{0}^{*}$ and the identity map on $V\langle A[t]\rangle$. By Lemma 4.2 .2 the pro-algebra $\mathcal{U}\left(V\langle A[t]\rangle, I_{V\langle A[t]\rangle}^{m}\right)_{\mathrm{lg}}$ is quasi-free. Then the homotopy invariance of the $X$-complex and homotopy inverse limits implies that $\mathbb{H} \mathbb{A}\left(\iota_{A}\right) \circ \mathbb{H} \mathbb{A}\left(\mathrm{ev}_{0}\right)$ is chain homotopic to $\mathbb{H} \mathbb{A}(\mathrm{id})$.

Next we prove (2). There is a canonical $V$-algebra lifting $V\left\langle M_{\Lambda}(A)\right\rangle \rightarrow$ $M_{\Lambda}(V\langle A\rangle)$ of the identity map on $M_{\Lambda} A$, induced by the obvious maps $V M_{\Lambda}(A) \rightarrow$ $M_{\Lambda}(V A) \rightarrow M_{\Lambda}(V\langle A\rangle)$. Let $I=\operatorname{ker}\left(V\left\langle M_{\Lambda}(A)\right\rangle \rightarrow M_{\Lambda}(A)\right)$ and $J=\operatorname{ker}(V\langle A\rangle \rightarrow$ $A)$. Then we get a pro-algebra homomorphism between tube algebras

$$
\mathcal{U}\left(V\left\langle M_{\Lambda}(A)\right\rangle, I^{\infty}\right) \rightarrow M_{\Lambda}\left(\mathcal{U}\left((V\langle A\rangle), J^{\infty}\right)\right) .
$$

The functorality of the $X$-complex gives a pro-chain map

$$
X\left(\mathcal{U}\left(V\left\langle M_{\Lambda}(A)\right\rangle, I^{\infty}\right)\right) \rightarrow X\left(M_{\Lambda}\left(\mathcal{U}\left((V\langle A\rangle), J^{\infty}\right)\right) .\right.
$$

We can now proceed as in the proof of [26 Theorem 5.65], adapted to the situation of [12. Example 6.3].

\section{Nilpotent extensions and excision}

6.1. Nilpotent resolutions of $\mathbb{F}$-algebras. In this section, we discuss analytically nilpotent resolutions that can be naturally associated to $\mathbb{F}$-algebras. Our motivation here is to prove that our theory satisfies excision for finitely generated $\mathbb{F}$ algebras. Specifically, we will show that an extension of finitely generated $\mathbb{F}$-algebras extends to an extension of pro-dagger algebras with analytically nilpotent kernels, and then appeal to Proposition 2.1.4 An important feature of our framework is that we do not require our $\mathbb{F}$-algebras to admit $V$-algebra lifts $R$ that satisfy $R / \pi R \cong A$. Instead, we resolve $A$ by an extension of tube algebras

$$
\mathcal{U}\left(I, I^{\infty}\right) \nrightarrow \mathcal{U}\left(\mathrm{T} R, I^{\infty}\right) \rightarrow A,
$$

where $I=\operatorname{ker}(\mathrm{T} R \rightarrow A)$, and $R$ is a $V$-module with a non-associative multiplication, that reduces $\bmod \pi$ to the multiplication on $A$. Such a lift $R$ always exists.

In what follows, let $A$ be an $\mathbb{F}$-algebra and let $R$ be a $V$-module. Let $\Omega^{l}(R):=$ $R^{+} \otimes R^{\otimes l}$ for $l>0$ and $\Omega^{0}(R)=R$ as $V$-modules, where $R^{+}:=R \oplus V$ is the $V$-algebra 
obtained by adjoining a unit to $R$. Let $\Omega(R)=\oplus_{l=0}^{\infty} \Omega^{l}(R), \Omega^{\mathrm{ev}}(R)=\oplus_{l=0}^{\infty} \Omega^{2 l}(R)$, and $\Omega^{\text {odd }}(R)=\oplus_{l=0}^{\infty} \Omega^{2 l+1}(R)$. We now define a $V$-module isomorphism

$$
\iota: \Omega^{\mathrm{ev}}(R) \stackrel{\cong}{\rightarrow} \mathrm{T} R,
$$

using a possibly non-associative $V$-bilinear multiplication $\mu: R \otimes R \rightarrow R$. Let $\otimes$ denote the multiplication in $\mathrm{T} R$. For $x_{1}, x_{2} \in R$, we define

$$
\iota\left(\mathrm{d} x_{1} \mathrm{~d} x_{2}\right):=\mu\left(x_{1}, x_{2}\right)-x_{1} \otimes x_{2} \in \mathrm{T} R .
$$

More generally,

$$
\begin{gathered}
\iota\left(\mathrm{d} x_{1} \mathrm{~d} x_{2} \ldots \mathrm{d} x_{2 l-1} \mathrm{~d} x_{2 l}\right):=\iota\left(\mathrm{d} x_{1} \mathrm{~d} x_{2}\right) \otimes \cdots \otimes \iota\left(\mathrm{d} x_{2 l-1} \mathrm{~d} x_{2 l}\right), \\
\iota\left(x_{0} \mathrm{~d} x_{1} \mathrm{~d} x_{2} \ldots \mathrm{d} x_{2 l-1} \mathrm{~d} x_{2 l}\right):=x_{0} \otimes \iota\left(\mathrm{d} x_{1} \mathrm{~d} x_{2}\right) \otimes \cdots \otimes \iota\left(\mathrm{d} x_{2 l-1} \mathrm{~d} x_{2 l}\right)
\end{gathered}
$$

for $x_{0}, \ldots, x_{2 l} \in R$. These maps $\Omega^{2 l}(R) \rightarrow \mathrm{T} R$ combine to a map $\iota: \Omega^{\mathrm{ev}}(R) \rightarrow \mathrm{T} R$.

Lemma 6.1.1. The map $\iota: \Omega^{\mathrm{ev}}(R) \rightarrow \mathrm{T} R$ is an isomorphism of $V$-modules.

Proof. We define filtrations on $\mathrm{T} R$ and $\Omega^{\mathrm{ev}} R$. Let $(\mathrm{T} R)_{j}$ be the $V$-submodule generated by $x_{1} \otimes \cdots \otimes x_{m}$ with $x_{i} \in R, m \leq j$. Let $\left(\Omega^{\mathrm{ev}} R\right)_{j}$ be the $V$-submodule generated by $\Omega^{2 k} R$ for all $k$ with $2 k<j$ and by the "closed" $2 k$-forms $\mathrm{d} x_{1} \ldots \mathrm{d} x_{2 k}$ if $2 k=j$. We prove by induction on $j$ that $\iota$ restricts to an isomorphism from $\left(\Omega^{\mathrm{ev}} R\right)_{j}$ onto $(\mathrm{T} R)_{j}$. The assertion is empty for $j=0$. Assuming it for $j-1 \geq 0$, we prove it for $j$. We have $(\mathrm{T} R)_{j} /(\mathrm{T} R)_{j-1} \cong R^{\otimes j}$. By definition, $\iota$ maps $\mathrm{d} x_{1} \ldots \mathrm{d} x_{2 k}$ and $x_{0} \mathrm{~d} x_{1} \ldots \mathrm{d} x_{2 k}$ to $(-1)^{k} x_{1} \otimes \cdots \otimes x_{2 l}$ and $(-1)^{k} x_{0} \otimes x_{1} \otimes \cdots \otimes x_{2 l}$ modulo shorter terms. So $\iota$ maps $\left(\Omega^{\mathrm{ev}} R\right)_{j}$ into $(\mathrm{T} R)_{j}$ and induces an isomorphism from $\left(\Omega^{\mathrm{ev}} R\right)_{j} /\left(\Omega^{\mathrm{ev}} R\right)_{j-1}$ onto $(\mathrm{T} R)_{j} /(\mathrm{T} R)_{j-1}$. By the induction assumption and the Five Lemma, it induces an isomorphism from $\left(\Omega^{\mathrm{ev}} R\right)_{j}$ onto $(\mathrm{T} R)_{j}$.

In the following, we identify $\mathrm{T} R$ and $\Omega^{\mathrm{ev}} R$ using this isomorphism. Let $\odot$ be the associative multiplication on $\Omega^{\mathrm{ev}} R$ that corresponds to the multiplication in $\mathrm{T} R$. If $\mu$ is associative, then $\odot$ is the well known Fedosov product, $\omega \odot \eta=\omega \eta-\mathrm{d} \omega \mathrm{d} \eta$, where we use the usual multiplication of differential forms dictated by the Leibniz rule (see [16]). When $\mu$ is non-associative, this has to be corrected by terms involving the associator of $\mu$. Writing $\mu$ multiplicatively, the basic rule is

$$
\left(\mathrm{d} x_{1} \mathrm{~d} x_{2}\right) \odot x_{3}-\mathrm{d} x_{1} \mathrm{~d}\left(x_{2} x_{3}\right)+\mathrm{d}\left(x_{1} x_{2}\right) \mathrm{d} x_{3}-x_{1} \mathrm{~d} x_{2} \mathrm{~d} x_{3}=\left(x_{1} x_{2}\right) x_{3}-x_{1}\left(x_{2} x_{3}\right)
$$

for all $x_{1}, x_{2}, x_{3} \in R$. The homomorphism $p: \mathrm{T} R \rightarrow A$ satisfies $p\left(\mathrm{~d} x_{1} \mathrm{~d} x_{2}\right)=0$ because $\mu$ lifts the multiplication in $A$. Hence it corresponds to the map $\Omega^{\mathrm{ev}} R \rightarrow A$, $\sum \omega_{2 n} \mapsto \omega_{0} \bmod \pi$ for $\omega_{2 n} \in \Omega^{2 n} R$. So

$$
I:=\operatorname{ker}(\mathrm{T} R \rightarrow A)=\pi R \oplus \bigoplus_{j=1}^{\infty} \Omega^{2 j} R .
$$

Lemma 6.1.4. If $m \in \mathbb{N}^{*}$, then

$$
\mathcal{U}\left(\mathrm{T} R, I^{m}\right)=\sum_{j=0}^{\infty} \pi^{-\lfloor j / m\rfloor} \Omega^{2 j} R, \quad \mathcal{U}\left(I, I^{m}\right)=I \oplus \sum_{j=1}^{\infty} \pi^{-\lfloor j / m\rfloor} \Omega^{2 j} R,
$$

Proof. To simplify the writing, we adjoin a unit element to T $R$ and allow $x_{0} \in R^{+}:=R \oplus V \cdot 1$ to treat forms with and without $x_{0}$ on an equal footing. Let $I^{(+)}:=I \oplus \pi \cdot V \subseteq R^{+}$, so that $\pi \in I^{(+)}$. We claim that this does not affect the tube algebra $\sum_{j=0}^{\infty} \pi^{-j} I^{m j}$. Let $I^{(m)}:=\sum_{j=1}^{m} \pi^{m-j} I^{j}$. Then induction shows that

$$
\left(I^{(+)}\right)^{m}:=(I \oplus \pi \cdot V)^{m}=I^{(m)} \oplus \pi^{m} \cdot V .
$$


And

$$
\sum_{j=1}^{\infty} \pi^{-j} I^{(m j)}=\sum_{j=1}^{\infty} \sum_{k=1}^{m j} \pi^{m j-k-j} I^{k}=\sum_{j=1}^{\infty} \pi^{-j} I^{m j}
$$

Let $m \geq 1$ and $j \geq 0$. Let $(m-j)_{+}$be $m-j$ if $m-j \geq 0$ and 0 otherwise. Let $x_{0} \in R^{+}$, $x_{1}, \ldots, x_{2 j} \in R$. Then

$$
\pi^{(m-j)_{+}} x_{0} d x_{1} \ldots \mathrm{d} x_{2 j}=\left(\pi^{(m-j)_{+}} x_{0}\right) \odot \mathrm{d} x_{1} \mathrm{~d} x_{2} \odot \cdots \odot \mathrm{d} x_{2 j-1} \mathrm{~d} x_{2 j}
$$

is a product of $j$ terms $\mathrm{d} x_{2 i-1} \mathrm{~d} x_{2 i} \in I$ and the term $\pi^{(m-j)_{+}} x_{0}$, which involves $(m-j)_{+} \geq m-j$ factors $\pi \cdot 1 \in I^{(+)}$. Thus $\pi^{(m-j)_{+}} x_{0} \mathrm{~d} x_{1} \ldots \mathrm{d} x_{2 j} \in I^{(m)}$. We prove by induction on $m$ that these elements generate $I^{(m)}$.

Let $m \geq 1$ and assume the assertion is shown for $I^{(m)}$. We must prove it for $I^{(m+1)}=I \odot I^{(m)}+\pi \cdot I^{(m)}$. The second summand is easy to handle. The first is generated by products $\pi x_{0} \odot \pi^{(m-j)_{+}} y_{0} \mathrm{~d} y_{1} \ldots \mathrm{d} y_{2 j}$ and $x_{0} \mathrm{~d} x_{1} \cdots \mathrm{d} x_{2 i} \odot \pi^{(m-j)_{+}} y_{0} \mathrm{~d} y_{1} \ldots \mathrm{d} y_{2 j}$ with $i>0$. The first type of product gives

$$
\begin{aligned}
& \pi x_{0} \odot \pi^{(m-j)_{+}} y_{0} \mathrm{~d} y_{1} \ldots \mathrm{d} y_{2 j} \\
& \quad=\pi^{1+(m-j)_{+}}\left(x_{0} y_{0}\right) \mathrm{d} y_{1} \ldots \mathrm{d} y_{2 j}-\pi^{1+(m-j)_{+}} \mathrm{d} x_{0} \mathrm{~d} y_{0} \mathrm{~d} y_{1} \ldots \mathrm{d} y_{2 j},
\end{aligned}
$$

which has the desired form. In the second type of product, we rewrite $x_{0} \mathrm{~d} x_{1} \cdots \mathrm{d} x_{2 i} \odot$ $\pi^{(m-j)_{+}} y_{0}$ using 6.1 .2 . All associators that appear here belong to $\pi R$ because $A=R / \pi R$ is associative. Hence we get a sum of forms that have non-zero degree or belong to $\pi R$. So each summand in the product has the desired form. This completes our description of $I^{(m)}$. The assertions on tube algebras follow immediately.

Lemma 6.1.4 implies $\mathcal{U}\left(I, I^{m}\right) \cap \mathrm{T} R=I$ for $m \geq 2$. Hence there is a natural extension of $V$-algebras

$$
\mathcal{U}\left(I, I^{m}\right) \nrightarrow \mathcal{U}\left(\mathrm{T} R, I^{m}\right) \rightarrow A
$$

for all $m \geq 2$. Letting $m$ vary, this becomes an extension of pro-algebras

$$
\mathcal{U}\left(I, I^{\infty}\right) \nrightarrow \mathcal{U}\left(\mathrm{T} R, I^{\infty}\right) \rightarrow A,
$$

where $A$ is viewed as a constant pro-algebra. Its kernel is nilpotent $\bmod \pi$ by 12. Proposition 4.2.3]. Lemma 6.1.4 also shows that $\bigcap_{m \in \mathbb{N}^{*}} \mathcal{U}\left(\mathrm{T} R, I^{m}\right)=\mathrm{T} R$ or, equivalently,

$$
\underset{\lim }{\longleftarrow} \mathcal{U}\left(\mathrm{T} R, I^{m}\right)=\mathrm{T} R \text {. }
$$

We may choose a representative in $R$ and then in TR for each element of $A$. So the extension 6.1.6 splits by a morphism of pro-sets. We cannot expect much more because $A$ is an $\mathbb{F}$-algebra, whereas $\mathcal{U}\left(\mathrm{T} R, I^{m}\right)$ is torsion-free for all $m \in \mathbb{N}^{*}$.

Equation (6.1.6) gives us a pro-algebra lifting of an $\mathbb{F}$-algebra with a nilpotent kernel. In order to be in the situation of Proposition 2.1.4, however, we need an extension of $A$ by a pro-dagger algebra and an analytically nilpotent kernel. To obtain such a resolution in a natural way, we take $R=V A$ as the free $V$-module over $A$, and equip $A$ and $R$ with the fine bornology. Equipping the tube algebra $\mathcal{U}\left(\mathrm{T} R, I^{\infty}\right)=\mathcal{U}\left(V\langle A\rangle, I^{\infty}\right)$ with the fine bornology, we now show that we can enlarge 6.1 .6 to an analytically nilpotent extension

$$
\mathcal{U}\left(I, I^{\infty}\right)^{\dagger} \rightarrow \mathcal{U}\left(\mathrm{T} R, I^{\infty}\right)^{\dagger} \rightarrow A .
$$

We first describe the quotient bornology of dagger complete tube algebras $\mathcal{U}\left(R, I^{\infty}\right)^{\dagger}$ by their canonical ideals $\mathcal{U}\left(I, I^{\infty}\right)^{\dagger}$. Here $R$ is any $V$-algebra presentation 
of an $\mathbb{F}$-algebra $A$ with $I=\operatorname{ker}(R \rightarrow A)$, and the tube algebras $\mathcal{U}\left(R, I^{m}\right)$ are equipped with the fine bornology for each $m \in \mathbb{N}^{*}$.

Proposition 6.1.7. The canonical map from $\mathcal{U}\left(I, I^{m}\right)^{\dagger}$ to $\mathcal{U}\left(R, I^{m}\right)^{\dagger}$ is a bornological embedding. Its image is a closed ideal. It induces an isomorphism

$$
\mathcal{U}\left(I, I^{m}\right)^{\dagger} \otimes F \cong \mathcal{U}\left(R, I^{m}\right)^{\dagger} \otimes F .
$$

The inclusions $\mathcal{U}\left(I, I^{m}\right) \rightarrow \mathcal{U}\left(I, I^{m}\right)^{\dagger}$ and $\mathcal{U}\left(R, I^{m}\right) \rightarrow \mathcal{U}\left(R, I^{m}\right)^{\dagger}$ induce an isomorphism

$$
\mathcal{U}\left(R, I^{m}\right) / \mathcal{U}\left(I, I^{m}\right) \cong \mathcal{U}\left(R, I^{m}\right)^{\dagger} / \mathcal{U}\left(I, I^{m}\right)^{\dagger} .
$$

The quotient $\mathcal{U}\left(R, I^{m}\right) / \mathcal{U}\left(I, I^{m}\right)$ is an $\mathbb{F}$-algebra with the fine bornology.

PROOF. We claim that the linear growth bornology of $\mathcal{U}\left(R, I^{m}\right)$ restricted to $\mathcal{U}\left(I, I^{m}\right)$ is the linear growth bornology of $\mathcal{U}\left(I, I^{m}\right)$. To prove this, we equip $\mathcal{U}\left(R, I^{m}\right)$ with the bornology generated by the inclusions of $\mathcal{U}\left(I, I^{m}\right)$ and $R$. That is, the bounded subsets of $\mathcal{U}\left(R, I^{m}\right)$ are those of the form $S+T$ where $S$ is a finitely generated $V$-submodule in $R$ and $T$ has linear growth in $\mathcal{U}\left(I, I^{m}\right)$. We claim that $\mathcal{U}\left(R, I^{m}\right)$ with this bornology is a semi-dagger algebra.

The restriction of our bornology on $\mathcal{U}\left(R, I^{m}\right)$ to $\mathcal{U}\left(I, I^{m}\right)$ is the linear growth bornology because $S \cap \mathcal{U}\left(I, I^{m}\right)$ for finitely generated $S \subseteq R$ is again finitely generated and hence bounded in $\mathcal{U}\left(I, I^{m}\right)$. The induced bornology on $A_{1}:=\mathcal{U}\left(R, I^{m}\right) /$ $\mathcal{U}\left(I, I^{m}\right)$ is the fine bornology. This quotient algebra is a quotient of the $\mathbb{F}$-algebra $A$ because $R / I \cong A$. This is semi-dagger because $\pi \cdot A=0$. Hence $\mathcal{U}\left(R, I^{m}\right)$ with our tailor-made bornology is an extension of semi-dagger algebras, hence semidagger. Thus our bornology contains all subsets of linear growth in $\mathcal{U}\left(R, I^{m}\right)$. Conversely, all subsets of the form $S+T$ as above have linear growth in $\mathcal{U}\left(R, I^{m}\right)$. So our new bornology on $\mathcal{U}\left(R, I^{m}\right)$ is just the linear growth bornology. Hence the linear growth bornology on $\mathcal{U}\left(R, I^{m}\right)$ restricts to the linear growth bornology on $\mathcal{U}\left(I, I^{m}\right)$ and induces the fine bornology on the quotient $A_{1}$. We also see that the inclusion $\mathcal{U}\left(I, I^{m}\right)_{\lg } \rightarrow \mathcal{U}\left(R, I^{m}\right)_{\lg }$ induces bornological isomorphisms $\mathcal{U}\left(I, I^{m}\right)_{\lg } \otimes F \cong \mathcal{U}\left(R, I^{m}\right)_{\lg } \otimes F$ and $\mathcal{U}\left(R, I^{m}\right)_{\lg } / \mathcal{U}\left(I, I^{m}\right)_{\lg } \cong A_{1}$.

Both $\mathcal{U}\left(I, I^{m}\right)$ and $\mathcal{U}\left(R, I^{m}\right)$ are bornologically torsion-free. This remains so for the linear growth bornologies by [12, Lemma 2.2.7]. Hence

$$
\mathcal{U}\left(I, I^{m}\right)^{\dagger} \otimes F=\overparen{\mathcal{U}\left(I, I^{m}\right)_{\lg } \otimes F}=\overparen{\mathcal{U}\left(R, I^{m}\right)_{\lg } \otimes F}=\mathcal{U}\left(R, I^{m}\right)^{\dagger} \otimes F .
$$

Since both $\mathcal{U}\left(I, I^{m}\right)^{\dagger}$ and $\mathcal{U}\left(R, I^{m}\right)^{\dagger}$ are bornologically torsion-free, they bornologically embed into $\widehat{\mathcal{U}\left(I, I^{m}\right)_{\lg } \otimes F}=\overparen{\mathcal{U}\left(R, I^{m}\right)_{\lg } \otimes F}$. Hence the canonical map $\mathcal{U}\left(I, I^{m}\right)^{\dagger} \rightarrow \mathcal{U}\left(R, I^{m}\right)^{\dagger}$ is a bornological embedding. Since $\mathcal{U}\left(I, I^{m}\right)^{\dagger}$ is complete, its image is closed. It is a two-sided ideal because $\mathcal{U}\left(I, I^{m}\right)$ is a two-sided ideal in $\mathcal{U}\left(R, I^{m}\right)$. Since the quotient $\mathcal{U}\left(R, I^{m}\right)_{\lg } / \mathcal{U}\left(I, I^{m}\right)_{\mathrm{lg}} \cong A_{1}$ is an $\mathbb{F}$-algebra with the fine bornology, it is already complete. The completion functor preserves cokernels because it is a left adjoint functor. Hence the isomorphism above implies $\overline{\mathcal{U}\left(R, I^{m}\right)_{\mathrm{lg}}} /$ $\overline{\mathcal{U}\left(I, I^{m}\right)_{\lg }} \cong A_{1}$. That is, $\mathcal{U}\left(R, I^{m}\right)^{\dagger} / \mathcal{U}\left(I, I^{m}\right)^{\dagger} \cong \mathcal{U}\left(R, I^{m}\right) / \mathcal{U}\left(I, I^{m}\right)$.

Corollary 6.1.8. Let $A$ be an $\mathbb{F}$-algebra with the fine bornology. Then we have an analytically nilpotent extension

$$
\mathcal{U}\left(I, I^{\infty}\right)^{\dagger} \nrightarrow \mathcal{U}\left(V\langle A\rangle, I^{\infty}\right)^{\dagger} \rightarrow A
$$

of pro-bornological algebras, that splits by a bounded pro-map of sets. 
Proof. Set $R=V\langle A\rangle$ with the fine bornology in Proposition 6.1.7 and then use Equation (6.1.6). The kernel $\mathcal{U}\left(I, I^{\infty}\right)^{\dagger}$ is analytically nilpotent as nilpotence $\bmod \pi$ is unaffected by dagger completion.

REMARK 6.1.9. In Corollary 6.1.8, we can replace the free algebra $V\langle A\rangle$ by the free algebra over a smaller set. Namely, let $S$ be a basis for $A$ and $R:=V S$. Then $V\langle A\rangle \cong \mathrm{T} R$. So Proposition 6.1.7 and Equation 6.1.6 yield an extension

$$
\mathcal{U}\left(I, I^{\infty}\right)^{\dagger} \nrightarrow \mathcal{U}\left(\mathrm{T} R, I^{\infty}\right)^{\dagger} \rightarrow A .
$$

6.2. An explicit analytic tensor algebra lifting. The analytic tensor algebras constructed from free algebras in Corollary 6.1.8 and Remark 6.1.9 are too large. Consequently, they do not give us any information about their structure, by way of explicit descriptions of their bornologies. In this section, we start with a finitely generated $\mathbb{F}$-algebra $A$, whose generating set we use to build a specific basis $S \subseteq A$. We then take the free $V$-module $R$ over $S$, and replace the fine bornology by a larger bornology $R_{\mathrm{lg}}$. It turns out that the linear growth bornology on the resulting tube algebra $\left(\mathcal{U}\left(\mathrm{T} R_{\mathrm{lg}}, I^{m}\right)\right)_{m \in \mathbb{N}^{*}}$ is easier to describe. The completion of the tube algebra in this bornology is dagger homotopy equivalent to $\mathcal{U}\left(\mathrm{T} R, I^{\infty}\right)^{\dagger}$.

Let $A$ be a finitely generated $\mathbb{F}$-algebra and let $S_{1}$ be a generating subset of $A$. We assume $S_{1}$ to be linearly independent. We shall describe a filtration on $A$ by $\mathbb{F}$-vector subspaces. Let $\mathcal{F}_{j} A$ be the $\mathbb{F}$-vector subspace of $A$ generated by elements of the form $s_{1} \ldots s_{l}$, where $s_{i} \in S$ for $1 \leq i \leq l \leq j$. Set $\mathcal{F}_{0} A=\{0\}$. We have

$$
\mathcal{F}_{j} A \cdot \mathcal{F}_{k} A \subseteq \mathcal{F}_{j+k} A, \quad \mathcal{F}_{n} A \subseteq \mathcal{F}_{n+1} A .
$$

So $\left(\mathcal{F}_{n} A\right)_{n \in \mathbb{N}}$ is an increasing filtration of $A$. Furthermore, $S_{1}$ is a basis for $\mathcal{F}_{1} A$. The set $S_{1}$ together with products of the form $s_{1} \cdot s_{2}$, with $s_{1}, s_{2} \in S_{1}$ generates $\mathcal{F}_{2} A$. Omitting some products if necessary, we obtain a basis $S_{2}$ for $\mathcal{F}_{2} A$ containing $S_{1}$. Continuing like this, for each $j \geq 1$, we obtain bases $S_{j}$ for $\mathcal{F}_{j} A$ containing $S_{j-1}$. Then $S=\cup_{j=1}^{\infty} S_{j}$ is a basis for $A$.

Let $R$ be the free $V$-module generated by the set $S$. By construction, $R / \pi R \cong A$, and $R$ inherits an increasing filtration $\mathcal{F}_{j} R:=V S_{j}$. Let $q: R \rightarrow A$ be the quotient map. Then $q\left(\mathcal{F}_{j} R\right)=\mathcal{F}_{j} A$ by construction. We shall now construct a map $\mu: R \otimes R \rightarrow R$ that reduces mod $\pi$ to the (associative) multiplication map $\mu_{0}: A \otimes_{\mathbb{F}} A \rightarrow A$ for the algebra $A$ in the sense that the following diagram commutes:

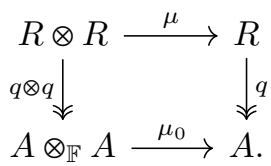

Let $x \in S_{j}$ and $y \in S_{k}$ for some $j$ and $k$. Then there is $z \in \mathcal{F}_{j+k} R$ such that $q(z)=\mu_{0}(q \otimes q(x \otimes y))$. We then set $\mu(x \otimes y):=z$. This map is well-defined as different representations of $\mu_{0}(x \otimes y)$ all map to $z$ for a specific choice of section $A \rightarrow R$. By construction, we have the following:

Lemma 6.2.1. The map $\mu: R \otimes R \rightarrow R$ satisfies $\mu\left(\mathcal{F}_{j} R \otimes \mathcal{F}_{k} R\right)=\mathcal{F}_{j+k} R$ for each $j$ and $k \in \mathbb{N}$.

Proof. By construction, we have

$$
\mu\left(\mathcal{F}_{j}(R) \otimes \mathcal{F}_{k}(R)\right) \subseteq \mathcal{F}_{j+k}(R) .
$$


When we divide out by $\pi$, the map $\mu$ is simply $\mu_{0}$, which restricts to a surjective $\operatorname{map} \mathcal{F}_{j}(A) \otimes_{\mathbb{F}} \mathcal{F}_{k}(A) \rightarrow \mathcal{F}_{j+k}(A)$. We have

$$
\mu_{0}\left(\mathcal{F}_{j}(A) \otimes_{\mathbb{F}} \mathcal{F}_{k}(A)\right) \cong \mu\left(\mathcal{F}_{j}(R) \otimes \mathcal{F}_{k}(R)\right) / \pi \cdot \mu\left(\mathcal{F}_{j}(R) \otimes \mathcal{F}_{k}(R)\right) .
$$

Here $\mathcal{F}_{j}(R) \otimes \mathcal{F}_{k}(R)$ and $\mathcal{F}_{j+k}(R)$ are finitely generated, free $V$-modules. So a $V$-module map between them is surjective once it is surjective mod $\pi$.

The multiplication map $\mu$ is in general non-associative and this means that we must modify the definition of the linear growth bornology for (associative) algebras. By Lemma 6.2.1, any element of $R$ may be written as a finite sum of products of elements in $\mathcal{F}_{1}(R)$. Conversely, an element belongs to $\mathcal{F}_{j}(R)$ if and only if we can get it using only products of length at most $j$. Here the way we put parentheses does not matter as they all give the same $V$-submodule in $R$. Hence the submodules of linear growth should be those of the form $\sum_{j=0}^{\infty} \pi^{j}\left(\mathcal{F}_{1}(R)\right)^{l(j+1)}=\sum_{j=0}^{\infty} \pi^{j} \mathcal{F}_{l(j+1)}(R)$ for some $l \in \mathbb{N}^{*}$. This leads to the following definition:

Definition 6.2.2. A subset of $R$ has linear growth if it is contained in a submodule of the form

$$
\sum_{j=0}^{\infty} \pi^{j} \mathcal{F}_{l(j+1)}(R)
$$

for some $l \in \mathbb{N}^{*}$. We denote $R$ with this bornology by $R_{\mathrm{lg}}$.

Lemma 6.2.3. Let $\lambda: \mathbb{N} \rightarrow \mathbb{N}$ be a function of linear growth, that is, $\lambda(n) \leq a n+b$ for some $a, b \in \mathbb{N}$. Then $\sum_{j=0}^{\infty} \pi^{j} \mathcal{F}_{\lambda(j)}(R)$ has linear growth in $R$.

Proof. Let $l>\max \{a, b\}$. Then $\lambda(j) \leq l(j+1)$, so that we have

$$
\sum_{j=0}^{\infty} \pi^{j} \mathcal{F}_{\lambda(j)}(R) \subseteq \sum_{j=0}^{\infty} \pi^{j} \mathcal{F}_{l(j+1)}(R)
$$

If $R$ is an associative algebra with the fine bornology, then this bornology is analogous to the usual definition of the linear growth bornology for algebras. By construction, $R_{\lg }$ is bornologically torsion-free and its reduction $\bmod \pi$ is $R / \pi R=A$ with the fine bornology. So $q: R \rightarrow A$ is a bornological quotient map. Completing $R$ with respect to this bornology yields a complete, bornologically torsion-free $V$-module $\overparen{R_{\mathrm{lg}}}$. Since the completion functor is a left adjoint functor and since an $\mathbb{F}$-algebra is already complete with respect to the fine bornology, we have

$$
\widetilde{R_{\mathrm{lg}}} / \pi \widetilde{R_{\mathrm{lg}}} \cong R / \pi R \cong A .
$$

So $\overparen{R_{\mathrm{lg}}} \rightarrow A$ is a bornological quotient map.

The construction above defines a bornologically torsion-free, bornological $V$ module $R_{\mathrm{lg}}$, whose bornology is described by a filtration $\left(\mathcal{F}_{j} R\right)_{j \in \mathbb{N}}$. We can use the filtration on $R$ to induce a filtration on differential forms $\Omega^{l}(R)$ as follows: let $\mathcal{F}_{j}\left(\Omega^{l}(R)\right)$ be the $V$-module generated by differential forms $x_{0} \mathrm{~d} x_{1} \cdots \mathrm{d} x_{l}$, where $x_{0} \in\left(\mathcal{F}_{j_{0}}(R)\right)^{+}, x_{i} \in \mathcal{F}_{j_{i}}(R)$ such that $\sum_{i=0}^{l} j_{i}=j$. This is an increasing filtration on the module $\Omega^{l}(R)$. We use the description of the tube algebra from Lemma 6.1.4. $\mathcal{U}\left(\mathrm{T} R, I^{m}\right)=\oplus_{j=0}^{\infty} \pi^{-\left\lfloor\frac{j}{m}\right\rfloor} \Omega^{2 j}(R)$ to equip it with the bornology $\mathcal{B}_{m}$ generated by $V$-modules of the form

$$
\sum_{k=0}^{\infty} \sum_{j=0}^{\infty} \pi^{-\left\lfloor\frac{j}{m}\right\rfloor+\left[\frac{j}{l}\right]+k} \mathcal{F}_{l(k+1)}\left(\Omega^{2 j}(R)\right),
$$


for some $l \in \mathbb{N}^{*}$.

Proposition 6.2.5. For each $m \in \mathbb{N}^{*}$, the bornological algebra $\left(\mathcal{U}\left(\mathrm{T} R, I^{m}\right), \mathcal{B}_{m}\right)$ is bornologically torsion-free and semi-dagger. So its completion is a dagger algebra. The ideal $\mathcal{U}\left(I, I^{m}\right)$ is bornologically closed, and the bornology $\mathcal{B}_{m}$ still induces the fine bornology on the quotient $\mathcal{U}\left(\mathrm{T} R, I^{m}\right) / \mathcal{U}\left(I, I^{m}\right) \cong A$.

Proof. We first show that $\left(\mathcal{U}\left(\mathrm{T} R, I^{m}\right), \mathcal{B}_{m}\right)$ is bornologically torsion-free. Let $S$ be a bounded subset in $\mathcal{B}_{m}$. Then there is an $\alpha$ such that

$$
\begin{array}{r}
S \subseteq \sum_{k=0}^{\infty} \sum_{j=0}^{\infty} \pi^{-\left\lfloor\frac{j}{m}\right\rfloor+\left\lceil\frac{j}{\alpha}\right\rceil+k} \mathcal{F}_{\alpha(k+1)}\left(\Omega^{2 j}(R)\right)=\sum_{j=0}^{\infty} \pi^{-\left\lfloor\frac{j}{m}\right\rfloor+\left\lceil\frac{j}{\alpha}\right\rceil} \mathcal{F}_{\alpha}\left(\Omega^{2 j}(R)\right) \\
+\pi \sum_{k=0}^{\infty} \sum_{j=0}^{\infty} \pi^{-\left\lfloor\frac{j}{m}\right\rfloor+\left\lceil\frac{j}{\alpha}\right\rceil+k} \mathcal{F}_{\alpha(k+2)}\left(\Omega^{2 j}(R)\right) .
\end{array}
$$

Then

$$
\pi^{-1} S \subseteq \sum_{j=0}^{\infty} \pi^{-\left\lfloor\frac{j}{m}\right\rfloor+\left\lceil\frac{j}{\alpha}\right\rceil-1} \mathcal{F}_{\alpha}\left(\Omega^{2 j}(R)\right)+\sum_{k=0}^{\infty} \sum_{j=0}^{\infty} \pi^{-\left\lfloor\frac{j}{m}\right\rfloor+\left\lceil\frac{j}{\alpha}\right\rceil+k} \mathcal{F}_{\alpha(k+2)}\left(\Omega^{2 j}(R)\right) .
$$

Denote the first term and the second term in the equation above by $T$ and $U$, respectively. The term $T$ is contained in

$$
\sum_{j=0}^{\infty} \pi^{-\left\lfloor\frac{j}{m}\right\rfloor+\left\lfloor\frac{j-1}{\alpha}\right\rfloor} \mathcal{F}_{\alpha}\left(\Omega^{2 j}(R)\right) \subseteq \pi^{-1} \mathcal{F}_{\alpha}(R)+\sum_{j=1}^{\infty} \pi^{-\left\lfloor\frac{j}{m}\right\rfloor+\left\lceil\frac{j}{2 \alpha}\right\rceil} \mathcal{F}_{2 \alpha}\left(\Omega^{2 j}(R)\right),
$$

which is bounded as $R_{\lg }$ is bornologically torsion-free and the latter term is a generator for the bornology $\mathcal{B}_{m}$. The second term $U$ is bounded in $\mathcal{B}_{m}$ by Lemma 6.2.3 Therefore, $\pi^{-1} S$ is bounded in $\mathcal{B}_{m}$.

To show that the bornology $\mathcal{B}_{m}$ is semi-dagger, we need the following estimate on the growth of the Fedosov product on $\Omega(R)$ :

LEMMA 6.2.6. Let $R$ be a $V$-module with a filtration $\left(\mathcal{F}_{l}(R)\right)_{l}$ by $V$-submodules. Then with the induced filtration on differential forms, we have

$$
\mathcal{F}_{l_{1}} \Omega^{2 i_{1}}(R) \odot \mathcal{F}_{l_{2}}\left(\Omega^{2 i_{2}}(R)\right) \subseteq \bigoplus_{j=0}^{1} \mathcal{F}_{l}\left(\Omega^{2 i+2 j}(R)\right) \oplus \bigoplus_{k=1}^{i_{1}} \pi^{k} \mathcal{F}_{l} \Omega^{2(i-k)}(R),
$$

where $i=i_{1}+i_{2}$ and $l=l_{1}+l_{2}$.

Proof. Let $\omega_{1}:=x_{0} \mathrm{~d} x_{1} \cdots \mathrm{d} x_{2 i_{1}}$ and $\omega_{2}:=y_{0} \mathrm{~d} y_{1} \cdots \mathrm{d} y_{2 i_{2}}$ be differential forms in $\mathcal{F}_{l_{1}} \Omega^{2 i_{1}}(R)$ and $\mathcal{F}_{l_{2}} \Omega^{2 i_{2}}(R)$. Since the Fedosov product is only determined by the action of $\omega_{1}$ on the 0 -th entry $y_{0}$ of $\omega_{2}$, it suffices to prove the case $i_{2}=0$. So we have $i=i_{1}$ and we rewrite $y_{0}=x_{2 i+1}$. We write $\omega_{1}$ as $i$ blocks of 2 -forms $x_{0} \odot \mathrm{d} x_{1} \mathrm{~d} x_{2} \odot \cdots \odot \mathrm{d} x_{2 i-1} \mathrm{~d} x_{2 i}$.

Let $a(x, y, z)=x(y z)-(x y) z$ denote the associator for the multiplication $\mu$ of $R$. Since the multiplication on $R$ is associative $\bmod \pi, a(x, y, z) \in \pi R$. Using the rule in 6.1.2, when we take the product $\omega_{1} \odot x_{2 i+1}$, we get a $2 i+2$-form $\mathrm{d} \omega_{1} \mathrm{~d} x_{2 i+1}$ and sums of $2 i$-forms

$$
x_{0} \mathrm{~d} x_{1} \mathrm{~d} x_{2} \cdots \mathrm{d}\left(x_{l} x_{l+1}\right) \cdots \mathrm{d} x_{2 i+1}
$$

that do not simplify further. We also get a decomposable $2 i$-form

$$
x_{0} \odot \cdots \mathrm{d} x_{2 i-3} \mathrm{~d} x_{2 i-2} \odot x_{2 i-1} \odot \mathrm{d} x_{2 i} \mathrm{~d} x_{2 i+1},
$$

and a $2 i-2$ form

$$
x_{0} \odot \mathrm{d} x_{1} \mathrm{~d} x_{2} \odot \cdots \odot a\left(x_{2 i-1}, x_{2 i}, x_{2 i+1}\right) .
$$


The $2 i$ and $2 i+2$-forms that do not decompose further are contained in $\mathcal{F}_{l}\left(\Omega^{2 i}(R)\right)$ and $\mathcal{F}_{l}\left(\Omega^{2 i+2}(R)\right)$, respectively.

Applying the product rule of the Fedosov product to the term $x_{0} \odot \mathrm{d} x_{1} \mathrm{~d} x_{2} \odot \cdots \odot$ $a\left(x_{2 i-1}, x_{2 i}, x_{2 i+1}\right)$ yields an indecomposable $2 i$-form in $\mathcal{F}_{l}\left(\Omega^{2 i}(R)\right)$, indecomposable sums of $2 i-2$-forms in $\pi \mathcal{F}_{l}\left(\Omega^{2 i-2}(R)\right)$, and a $2 i$-4-form

$$
x_{0} \odot \mathrm{d} x_{1} \mathrm{~d} x_{2} \odot \cdots \odot a\left(x_{2 i-3}, x_{2 i-2}, a\left(x_{2 i-1}, x_{2 i}, x_{2 i+1}\right)\right) \in \pi^{2} \mathcal{F}_{l}\left(\Omega^{2 i-4}(R)\right) .
$$

The same argument for the term $x_{0} \odot \cdots \odot \mathrm{d} x_{2 i-3} \mathrm{~d} x_{2 i-2} \odot x_{2 i-1} \odot \mathrm{d} x_{2 i} \mathrm{~d} x_{2 i+1}$ yields sums of $2 i$-forms that do not decompose further, a $2 i$-form

$$
x_{0} \odot \mathrm{d} x_{1} \mathrm{~d} x_{2} \odot \cdots \odot x_{2 i-3} \odot \cdots \odot \mathrm{d} x_{2 i} \mathrm{~d} x_{2 i+1},
$$

and a $2 i-2$-form

$x_{0} \odot \mathrm{d} x_{1} \mathrm{~d} x_{2} \odot \cdots \odot \mathrm{d} x_{2 i-5} \mathrm{~d} x_{2 i-4} \odot a\left(x_{2 i-3}, x_{2 i-2}, x_{2 i-1}\right) \odot \mathrm{d} x_{2 i} \mathrm{~d} x_{2 i+1} \in \pi \mathcal{F}_{l}\left(\Omega^{2 i-2}(R)\right)$.

So we see that each $2 i-2$-form arises precisely as an indecomposable term in the Fedosov expansion of a term of the form

$$
x_{0} \odot \cdots \odot a\left(x_{2 i-(2 j+1)}, x_{2 i-2 j}, x_{2 i-(2 j-1)}\right) \odot \cdots \odot \mathrm{d} x_{2 i} \mathrm{~d} x_{2 i+1} \in \pi \mathcal{F}_{l}\left(\Omega^{2 i-2}(R)\right),
$$

with $0 \leq j \leq i-1$. Iterating the same argument, we see that any $2 i-2 k$-form arises as an indecomposable term in the Fedosov expansion of a term of the form

$$
x_{0} \odot \cdots \odot a\left(x_{2 i-(2 j+1)}, x_{2 i-2 j}, a\left(x_{2 i-(2 j-1)}, x_{2 i-(2 j+3)}, a(\ldots)\right)\right) \odot \cdots,
$$

where the number of times the associator term occurs is $k$. And such a term must lie in $\pi^{k} \mathcal{F}_{l}\left(\Omega^{2 i-2 k}(R)\right)$.

Now let $T_{\alpha}=\sum_{j=0}^{\infty} \sum_{k=0}^{\infty} \pi^{-\lfloor j / m]+[j / \alpha]+k} \mathcal{F}_{\alpha(k+1)} \Omega^{2 j}(R)$ be a subset in $\mathcal{B}_{m}$ for some $\alpha \in \mathbb{N}^{*}$. We claim that $\pi T_{\alpha}^{2} \subseteq T_{\alpha}$. The $V$-module $\pi T_{\alpha}^{2}$ is generated by elements of the form

$$
\pi^{-\left\lfloor j_{1} / m\right\rfloor-\left\lfloor j_{2} / m\right\rfloor+\left[j_{2} / \alpha\right]+\left[j_{2} / \alpha\right]+k_{1}+k_{2}+1} \mathcal{F}_{\alpha\left(k_{1}+1\right)} \Omega^{2 j_{1}}(R) \odot \mathcal{F}_{\alpha\left(k_{2}+1\right)} \Omega^{2 j_{2}}(R),
$$

for $j_{1}, j_{2}, k_{1}, k_{2} \geq 0$. By Lemma 6.2.6.

$$
\begin{aligned}
& \mathcal{F}_{\alpha\left(k_{1}+1\right)} \Omega^{2 j_{1}}(R) \odot \mathcal{F}_{\alpha\left(k_{2}+1\right)} \Omega^{2 j_{2}}(R) \\
& \subseteq \mathcal{F}_{\alpha\left(k_{1}+k_{2}+2\right)} \Omega^{2\left(j_{1}+j_{2}\right)}(R) \oplus \mathcal{F}_{\alpha\left(k_{1}+k_{2}+2\right)} \Omega^{2\left(j_{1}+j_{2}+1\right)}(R) \\
& \oplus \bigoplus_{l=0}^{j_{1}} \pi^{l} \mathcal{F}_{\alpha\left(k_{1}+k_{2}+2\right)} \Omega^{2\left(j_{1}+j_{2}-l\right)}(R) .
\end{aligned}
$$

So $\pi T_{\alpha}^{2}$ is contained in sums of $V$-modules of the form

$$
T_{\alpha, 1}=\sum_{j_{1}, j_{2}=0}^{\infty} \sum_{k_{1}, k_{2}=0}^{\infty} \pi^{-\left\lfloor j_{1} / m\right\rfloor-\left\lfloor j_{2} / m\right\rfloor+\left\lceil j_{2} / \alpha\right]+\left\lceil j_{2} / \alpha\right]+k_{1}+k_{2}+1} \mathcal{F}_{\alpha\left(k_{1}+k_{2}+2\right)} \Omega^{2 n}(R),
$$

and

$$
T_{\alpha, 2}=\pi^{-\left\lfloor j_{1} / m\right\rfloor-\left\lfloor j_{2} / m\right\rfloor+\left[j_{2} / \alpha\right]+\left[j_{2} / \alpha\right]+l+k_{1}+k_{2}+1} \mathcal{F}_{\alpha\left(k_{1}+k_{2}+2\right)} \Omega^{2 t}(R),
$$

where $n=j_{1}+j_{2}$ or $j_{1}+j_{2}+1, t=j_{1}+j_{2}-l$, and $l$ varies between 1 and $j_{1}$.

If $n=j_{1}+j_{2}$, then the estimates $-\left\lfloor j_{1} / m\right\rfloor-\left\lfloor j_{2} / m\right\rfloor \geq-\lfloor n / m\rfloor$ and $\left\lceil j_{1} / \alpha\right\rceil+\left\lceil j_{2} / \alpha\right\rceil \geq$ $\lceil n / \alpha\rceil$ imply that $T_{\alpha, 1} \subseteq \sum_{n=0}^{\infty} \sum_{k=0}^{\infty} \pi^{-\lfloor n / m\rfloor+\lfloor n / \alpha\rfloor+k} \mathcal{F}_{\alpha(k+1)} \Omega^{2 n}(R)=T_{\alpha}$. Similarly, if $n=j_{1}+j_{2}+1$, the same estimates on the exponent of $\pi$ show that

$$
T_{\alpha, 1} \subseteq \sum_{n=1}^{\infty} \sum_{k=0}^{\infty} \pi^{-\left\lfloor\frac{n-1}{m}\right\rfloor+\left\lceil\frac{n-1}{\alpha}\right\rceil+k} \mathcal{F}_{\alpha(k+1)} \Omega^{2 n}(R) \subseteq T_{\alpha} .
$$


Now let $1 \leq l \leq j_{1}$ be fixed. Then

$$
-\left\lfloor j_{1} / m\right\rfloor-\left\lfloor j_{2} / m\right\rfloor+\left\lceil j_{1} / \alpha\right\rceil+\left\lceil j_{2} / \alpha\right\rceil+l \geq-\left\lfloor\frac{t+l}{m}\right\rfloor+\left\lceil\frac{t+l}{\alpha}\right\rceil+l \geq-\left\lfloor\frac{t}{m}\right\rfloor+\left\lceil\frac{t}{\alpha}\right\rceil,
$$

so $T_{\alpha, 2} \subseteq \sum_{t=0}^{\infty} \sum_{k=0}^{\infty} \pi^{-\left\lfloor\frac{t}{m}\right\rfloor+\left[\frac{t}{\alpha}\right]+k} \mathcal{F}_{\alpha(k+1)} \Omega^{2 t}(R)=T_{\alpha}$. Hence $\pi T_{\alpha}^{2} \subseteq T_{\alpha}$, so $\mathcal{B}_{m}$ is semi-dagger.

The ideal $\mathcal{U}\left(I, I^{m}\right)$ with the subspace bornology inherits bornological torsionfreeness from $\left(\mathcal{U}\left(\mathrm{T} R_{\mathrm{lg}}, I^{m}\right), \mathcal{B}_{m}\right)$. And, $\left(\mathcal{U}\left(I, I^{m}\right), \mathcal{B}_{m}\right) \rightarrow\left(\mathcal{U}\left(\mathrm{T} R_{\mathrm{lg}}, I^{m}\right), \mathcal{B}_{m}\right)$ is a bornological embedding. Since their quotient is an $\mathbb{F}$-algebra, tensoring with $F$ yields a bornological isomorphism

$$
\left.\left.\mathcal{U}\left(I, I^{m}\right), \mathcal{B}_{m}\right) \otimes F \cong \mathcal{U}\left(\mathrm{T} R_{\mathrm{lg}}, I^{m}\right), \mathcal{B}_{m}\right) \otimes F .
$$

By 27 , the completions $\overline{\left(\mathcal{U}\left(I, I^{m}\right), \mathcal{B}_{m}\right)}$ and $\overline{\left(\mathcal{U}\left(\mathrm{T} R_{\mathrm{lg}}, I^{m}\right), \mathcal{B}_{m}\right)}$ are bornologically torsion-free. Hence, $\overline{\left(\mathcal{U}\left(I, I^{m}\right), \mathcal{B}_{m}\right)}$ and $\overline{\left(\mathcal{U}\left(\mathrm{T} R_{\mathrm{lg}}, I^{m}\right), \mathcal{B}_{m}\right)}$ both embed into

$$
\overline{\left(\mathcal{U}\left(I, I^{m}\right), \mathcal{B}_{m}\right)} \otimes F \cong \overline{\left(\mathcal{U}\left(\mathrm{T} R_{\lg }, I^{m}\right), \mathcal{B}_{m}\right)} \otimes F .
$$

So the canonical map $\overline{\left(\mathcal{U}\left(I, I^{m}\right), \mathcal{B}_{m}\right)} \rightarrow \overline{\left(\mathcal{U}\left(\mathrm{T} R_{\mathrm{lg}}, I^{m}\right), \mathcal{B}_{m}\right)}$ is a bornological embedding. Since $\overline{\left(\mathcal{U}\left(I, I^{m}\right), \mathcal{B}_{m}\right)}$ is complete, its image is closed, as required.

Finally, we show that the quotient bornology on $\mathcal{U}\left(\mathrm{T} R_{\mathrm{lg}}, I^{m}\right) / \mathcal{U}\left(I, I^{m}\right)$ is the fine bornology. Any finite-dimensional subspace of $A$ is contained in some $\mathbb{F}$-vector subspace of the form $\mathcal{F}_{k}(A)$ for some $k \in \mathbb{N}$. This lifts to the bounded $V$-submodule $\mathcal{F}_{k}(R)$ by construction. We have $\mathcal{F}_{k}(R) \subseteq \sum_{i=0}^{\infty} \pi^{i} \mathcal{F}_{k+i}(R)$, which is bounded in the "linear growth bornology" on $R$ and hence in $\mathcal{B}_{m}$ for each $m \geq 1$. So the quotient map $\mathcal{U}\left(\mathrm{T} R_{\mathrm{lg}}, I^{m}\right) \rightarrow A$ is bounded in the fine bornology on $A$. Since an $\mathbb{F}$-algebra with the fine bornology is complete, and since the completion functor commutes with quotients, we obtain bornological isomorphisms

$$
\overline{\left(\mathcal{U}\left(\mathrm{T} R_{\mathrm{lg}}, I^{m}\right), \mathcal{B}_{m}\right)} / \overline{\left(\mathcal{U}\left(I, I^{m}\right), \mathcal{B}_{m}\right)} \cong A,
$$

completing the proof.

In summary, the above construction tells us that given a finitely generated $\mathbb{F}$-algebra $A$, we can construct a pro-dagger algebra $\mathcal{T}_{R} A:=\overline{\left(\mathcal{U}\left(\mathrm{T} R_{\mathrm{lg}}, I^{m}\right), \mathcal{B}_{m}\right)}$ that lifts $A$. Furthermore, the dagger algebra lifting respects bornologies in the sense that the quotient map $\mathcal{T}_{R} A \rightarrow A$ is bounded with respect to the fine bornology on A.

6.3. The excision theorem. In this section, we use our theory of nilpotent extensions to show that our homology theory satisfies excision. Let

$$
K \stackrel{i}{\rightarrow} E \stackrel{p}{\rightarrow} Q
$$

be an extension of finitely generated $\mathbb{F}$-algebras. We view them as bornological $V$-algebras with the fine bornology. We will prove that there is a long exact sequence in analytic cyclic homology:

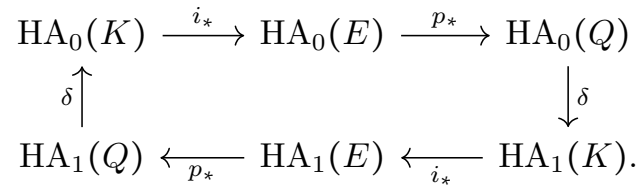


LEMMA 6.3.2. Suppose we have a morphism of extensions of the form

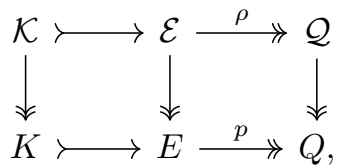

where the top row is a projective system of extensions of dagger algebras. Suppose further that the algebras in the bottom row carry the fine bornology, and that the kernels of the morphisms from the top row to the bottom row are analytically nilpotent. Then there exists a long exact sequence as in Equation 6.3.1.

Proof. By Proposition 4.2.3 the algebras in the top row compute the homology of the $\mathbb{F}$-algebras in the bottom row, since by hypothesis, the reduction mod $\pi$ has the fine bornology. We then use Proposition 2.1.4 and the Excision Theorem ([12, Theorem 5.1]) for pro- $V$-algebras.

Now given an extension of finitely generated $\mathbb{F}$-algebras $(6.3)$, we pick bases $S, T$ and non-associative liftings $M=V S$ and $N=V T$ of $E$ and $Q$ in the manner described in Section 6.2. By the universal property of free modules, an $\mathbb{F}$-linear map $p: E \rightarrow Q$ extends uniquely to a $V$-module map $\hat{p}: M \rightarrow N$. If $p: E \rightarrow Q$ is bounded in the fine bornology, then since the free lifts $M$ and $N$ are filtration preserving, the map $\hat{p}$ is bounded in the fine bornology on $M$ and $N$. This remains true for the linear-growth bornologies on $M$ and $N$. So we obtain a bounded $V$-module map $M \rightarrow N$ with a bounded $V$-module section $s: N \rightarrow M$. By functoriality of the tensor algebra, we obtain a bounded $V$-algebra homomorphism $\mathrm{T} M \rightarrow \mathrm{T} N$ that splits by the following $V$-module section

$$
\sigma\left(x_{0} \mathrm{~d} x_{1} \ldots \mathrm{d} x_{2 n}\right):=s\left(x_{0}\right) \mathrm{d} s\left(x_{1}\right) \ldots \mathrm{d} s\left(x_{2 n}\right)
$$

for all $x_{0}, x_{2 n+1} \in N^{+}$and $x_{i} \in N$ for $1 \leq i \leq 2 n$. Furthermore, $s$ maps even differential forms to even differential forms. Lemma 6.1.4 shows that they define linear maps on tube algebras $\sum_{j=0}^{\infty} \pi^{-\lfloor j / m\rfloor} \Omega^{2 j}(N) \rightarrow \sum_{j=0}^{\infty} \pi^{-\lfloor j / m\rfloor} \Omega^{2 j}(M)$.

LEMMA 6.3.3. The $V$-module section $\sigma: \mathcal{U}\left(\mathrm{T} N, I_{N}^{m}\right) \rightarrow \mathcal{U}\left(\mathrm{T} M, I_{M}^{m}\right)$ is bounded in the bornology defined in 6.2.4.

Proof. Since the section $s: N \rightarrow M$ is bounded, for each $k$, there is an $l \in \mathbb{N}$ with $s\left(\mathcal{F}_{k}(N)\right) \subseteq \mathcal{F}_{l}(M)$. Let $\omega=x_{0} \mathrm{~d} x_{1} \cdots \mathrm{d} x_{2 j} \in \mathcal{F}_{k}\left(\Omega^{2 j}(N)\right)$. Then $\sigma(\omega)=$ $s\left(x_{0}\right) \mathrm{d}\left(s\left(x_{1}\right)\right) \cdots \mathrm{d}\left(s\left(x_{2 j}\right)\right) \in \mathcal{F}_{l_{i}} \mathrm{~d}\left(\mathcal{F}_{l_{1}}(M)\right) \cdots \mathrm{d}\left(\mathcal{F}_{l_{2 j}}(M)\right) \subseteq \mathcal{F}_{l}\left(\Omega^{2 j}(M)\right)$, for $l=l_{0}+$ $\cdots+l_{2 j}$. So

$$
\sigma\left(\sum_{j} \sum_{k} \pi^{-\lfloor j / m\rfloor+k} \mathcal{F}_{k} \Omega^{2 j}(N)\right) \subseteq \sum_{l} \pi^{-\lfloor j / m\rfloor+l} \mathcal{F}_{l} \Omega^{2 j}(M)
$$

By Lemma 6.3.3 the section $\sigma$ extends to completions and yields a section for the canonical bounded algebra homomorphism $\rho: \mathcal{T}_{M} E \rightarrow \mathcal{T}_{N} Q$.

What we have therefore proved is the following:

Proposition 6.3.4. An extension $K \nrightarrow E \rightarrow Q$ of finitely generated $\mathbb{F}$-algebras with the fine bornology extends to a semi-split extension of pro-dagger algebras 


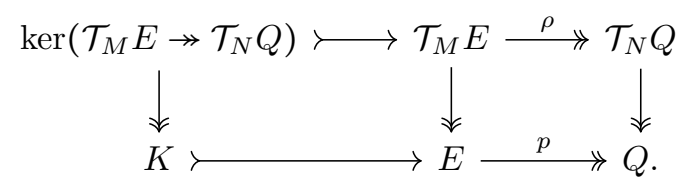

Furthermore, the quotient maps from the dagger algebras $\operatorname{ker}\left(\mathcal{T}_{M} E \rightarrow \mathcal{T}_{N} Q\right)$, $\mathcal{T}_{M} E \rightarrow E$ and $\mathcal{T}_{N} Q \rightarrow Q$ are bounded with respect to the fine bornologies on $K, E$ and $Q$.

Proof. By Proposition 6.2.5, for any $\mathbb{F}$-algebra $A$ with the fine bornology, there is a bornological quotient map from the dagger algebra $\mathcal{T}_{R} A$ to $A$, where $R$ is a filtered non-associative lifting. This, coupled with the discussion preceeding this theorem, shows that any bounded $\mathbb{F}$-algebra homomorphism $E \rightarrow Q$ lifts to a bounded $V$-algebra homomorphism $\mathcal{T}_{M} E \rightarrow \mathcal{T}_{N} Q$. Finally, the kernel $\mathcal{T}_{M} E \rightarrow \mathcal{T}_{N} Q$ is a pro-subalgebra of a pro-dagger algebra and is hence a dagger algebra. The induced map to $K$ is bounded in the fine bornology. Finally, it splits since the pro-set section $E \rightarrow \mathcal{T}_{M} E$ comes from a set-theoretic section $E \rightarrow M$, which in turn restricts to a section $K \rightarrow \operatorname{ker}(M \rightarrow N) \rightarrow \operatorname{ker}\left(\mathcal{T}_{M} E \rightarrow \mathcal{T}_{N} Q\right)$.

By Lemma 6.3.2 the lifted pro-algebras $\operatorname{ker}\left(\mathcal{T}_{M} E \rightarrow \mathcal{T}_{N} Q\right), \mathcal{T}_{M} E$ and $\mathcal{T}_{N} Q$ are all admissible liftings of $K, E$ and $Q$, that is, they compute their analytic cyclic homologies. This completes the proof of excision for analytic cyclic homology for finitely generated $\mathbb{F}$-algebras.

REMARK 6.3.5. The methods in this section should also help us prove excision for extensions of countably generated $\mathbb{F}$-algebras. This would entail building a non-associative lifting $R$ from a countable generating set for the $\mathbb{F}$-algebra $A$ we start with. The lifting $R$ should then be an inductive limit of finitely generated non-associative algebras, for which we can again build tube-tensor algebras with explicit semi-dagger bornologies. However, we do not treat this more general version of excision here. 


\section{CHAPTER 4}

\section{Conclusion and outlook}

The results in this thesis achieve three main things. First, they formalise Monsky and Washnitzer's weak completion using bornologies, which is the framework for our homology theories. Second, we construct a homology theory that satisfies certain desirable formal properties. Third, we provide computations of our homology theory for certain classes of algebras that are interesting in non-commutative geometry and algebraic geometry. In particular, for algebras corresponding to smooth 1dimensional varieties over a finite field, our homology theory agrees with rigid cohomology.

\section{What is still missing?}

The main result of $[\mathbf{1 1}$ shows that for finitely generated, commutative $\mathbb{F}$ algebras, periodic cyclic homology coincides with rigid cohomology. In [12], we have shown that for smooth curves (that is, one-dimensional affine varieties), our theory coincides with periodic cyclic homology and hence, rigid cohomology. This is because in Hochschild cohomological dimension 1, smooth algebras are the same as quasi-free algebras, for which the $X$-complex computes the analytic and the periodic cyclic theories.

In general, if $R$ is a smooth commutative $V$-algebra, it has Hochschild cohomological dimension $n$, for some $n \in \mathbb{N}$. Using standard results from homological perturbation theory, one can then show that a certain projection

$$
\mathbb{H} \mathbb{P}\left(R^{\dagger} \otimes F\right) \rightarrow X^{n}\left(R^{\dagger} \otimes F\right)
$$

of the periodic cyclic complex is a chain homotopy equivalence. We refer the reader to [26. Definition A.122, Theorem A.123] for the definition of the $X^{n}$-complex and a proof of this result. Viewing the analytic chain complex as the $b-B$-bicomplex $(\mathbb{H} \mathbb{P}, b+B)$, with a certain analytic bornology, we can show that there is a bounded projection map

$$
\mathbb{H} \mathbb{A}\left(R^{\dagger}\right) \rightarrow X^{n}\left(R^{\dagger} \otimes F\right) \sim \mathbb{H} \mathbb{P}\left(R^{\dagger} \otimes F\right) .
$$

For Banach algebras over $\mathbb{C}$ with the von Neumann bornology, results by Masoud Khalkhali (see $\mathbf{2 2}$. Theorem 2.50]) can be used to show that if $A$ has finite cohomological dimension $n$, then $\mathbb{H} \mathbb{A}(A) \rightarrow X^{n}(A)$ is a chain homotopy equivalence. The proof of this result uses an explicit formula for the contracting homotopy of the kernel of the projection map $\mathcal{F}_{n}(\mathbb{H} \mathbb{P}(A))=\operatorname{ker}\left(\mathbb{H} \mathbb{P}(A) \rightarrow X^{n}(A)\right)$. However, in our case, it is not obvious why such contracting homotopies should be bounded in the analytic bornology on the complex $\mathbb{H} \mathbb{A}\left(R^{\dagger}\right)$. The comparison of the analytic and periodic cyclic theories in the non-Archimedean setting is therefore an important issue that this thesis has not resolved in full generality. 


\section{Some future directions}

We now discuss some future projects that I plan to pursue, using the tools developed in this thesis.

2.1. More on liftings $\bmod \pi$. The main result in Chapter 3 says that if $D$ is any dagger algebra lifting, then $\mathrm{HA}_{*}(D) \cong \mathrm{HA}_{*}(D / \pi D)$, provided $D / \pi D$ has the fine bornology. An important question now is how such liftings arise and are related to $\pi$-adic completions, which seem more "canonical 1 .

Let $R$ be a $\pi$-adically complete $V$-algebra and let $A:=R / \pi R$. We give $R$ the compactoid bornology: a subset $S \subseteq R$ is bounded if and only if for every $n \in \mathbb{N}$, there is a finite set $F \subseteq R$ such that $S \subseteq F+\pi^{n} R$. This bornology appears in connection with the work of Bambozzi, Ben Bassat and Kremnitzer on derived analytic geometry (see $[7$ ). It can then be shown that $R$ with the compactoid bornology is a dagger algebra. Furthermore, the analysis results from Section 4.1 yield the following:

Lemma. Let $M$ be a complete, torsion-free bornological $V$-module. Then $M / \pi M$ has the fine bornology if and only if the bornology on $M$ is compactoid, that is, for any bounded submodule $N \subseteq M$, there is a bounded compactoid submodule $N^{\prime}$ that contains $N$.

The lemma above can be used to feed a $\pi$-adically complete bornological algebra $R$ with the compactoid bornology into the statement of Proposition 2.1 .8 to yield $\mathrm{HA}_{*}(R) \cong \mathrm{HA}_{*}(A)$. So if $R$ is any reasonable $V$-algebra lifting mod $\pi$ of $A$, and $A$ has the fine bornology, then we expect the following:

TheOREM. With $R$ and $A$ as above, we have

$$
\mathrm{HA}_{*}\left(R^{\dagger}\right) \cong \mathrm{HA}_{*}(A) \cong \mathrm{HA}_{*}(\widehat{R}) .
$$

Another result we expect due to Proposition 2.1.8 is the invariance of analytic

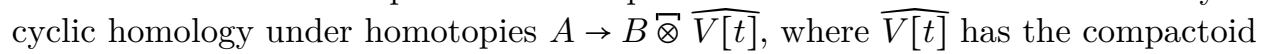
bornology and $A$ and $B$ are dagger algebras whose reductions mod $\pi$ have the fine bornology. This should hold because of the following:

TheOREM. Let $B$ be a dagger algebra whose reduction mod $\pi$ has the fine bornology. Then $\mathrm{HA}_{*}(B) \cong \mathrm{HA}_{*}(B \bar{\otimes} \overline{V[t]})$.

Proof. The proof follows from a string of isomorphisms:

$$
\begin{aligned}
\mathrm{HA}_{*}(B \bar{\otimes} \overline{V[t]}) \cong \mathrm{HA}_{*}(B \bar{\otimes} \overline{V[t]} / \pi(B \otimes \overline{V[t]})) \\
\\
\cong \mathrm{HA}_{*}(B / \pi B \otimes \mathbb{F}[t]) \cong \mathrm{HA}_{*}(B / \pi B) \cong \mathrm{HA}_{*}(B) .
\end{aligned}
$$

These results will be added to the manuscript of Chapter 3

\footnotetext{
${ }^{1}$ I am thankful to the organisers for inviting me to the Oberwolfach Workshop Non-commutative geometry and cyclic homology, where these ideas were first discussed.
} 
2.2. Product structures in non-Archimedean cyclic homology. Here we consider the behaviour of analytic cyclic homology with tensor products. In periodic cyclic homology, and archimedean versions of analytic and local cyclic homology, such results are due to Michael Puschnigg. Briefly, if $\mathbb{H}$ denotes any of the chain complexes of any of these theories, we have under certain conditions chain homotopy equivalences

$$
\mathbb{H}(A \otimes B) \sim \mathbb{H}(A) \otimes \mathbb{H}(B),
$$

that are associative, symmetric and monoidal in a suitable sense. If $\mathbb{H}=\mathbb{H} \mathbb{P}$, then the above holds for any pro-algebra $A$ and $B$. But for $\mathbb{H} \mathbb{A}$, we need that the algebras $A$ and $B$ are locally multiplicative in the sense that they are direct limits of semi-normed subalgebras. Such results are used to construct an exterior product operation on bivariant versions of these theories.

In the non-archimedean theory for torsion-free $V$-algebras, I expect a similar result to hold, stated as follows:

Theorem. Let $A$ and $B$ be complete, bornologically torsion-free unital $V$ algebras. Then there is a chain homotopy equivalence $\mathbb{H} \mathbb{A}(A \otimes B) \sim \mathbb{H} \mathbb{A}(A) \otimes \mathbb{H} \mathbb{A}(B)$.

To see why this could be true, we first observe that the identity map on the algebra $A \bar{\otimes} B$ extends to a morphism $\mathcal{T}(A \bar{\otimes} B) \rightarrow \mathcal{T}(A) \otimes \mathcal{T}(B)$ of analytically nilpotent extensions. The proof that the kernel of $\mathcal{T} A \otimes \mathcal{T} B \rightarrow A \bar{\otimes} B$ is analytically nilpotent uses $\mathbf{1 2}$, Proposition 4.2.5]. It is precisely here that the assumption of local multiplicativity is used in analytic cyclic homology for $\mathbb{C}$-algebras. So invariance under analytically nilpotent extensions gives

$$
\mathbb{H} \mathbb{A}(A \otimes B) \sim \mathbb{H} \mathbb{A}(\mathcal{T} A \bar{\otimes} \mathcal{T} B) .
$$

Now a typical issue arises involving homological dimensions. In the entire theory developed in this thesis, analytic cyclic homology is mostly only easily computable in cohomological dimension 1. However, $\mathcal{T} A \otimes \mathcal{T} B$ in general has cohomological dimension 2. In dimension 2, periodic cyclic homology is chain homotopy equivalent to a certain quotient of it, namely the $X^{2}$-complex, which also came up in the proof of homotopy invariance of our theory (see Proposition 4.6.1). So the task is to show that there is a chain homotopy equivalence

$$
\mathbb{H} \mathbb{A}(\mathcal{T} A \bar{\otimes} \mathcal{T} B) \sim X^{2}(\mathcal{T} A \otimes \mathcal{T} B) \otimes F .
$$

In the Archimedean case, Puschnigg (see [31]) constructs certain explicit maps at the level of the tensor algebras, that implement the chain homotopy equivalence above. We hope that similar formulas can also be used in our setting. Suppose we can adapt Puschnigg's methods, the rest of the proof goes as expected:

$$
X^{2}(\mathcal{T} A \bar{\otimes} B) \bar{\Phi} F \sim X(\mathcal{T} A \Phi F) \otimes X(\mathcal{T} B \bar{\otimes} F)=\mathbb{H} \mathbb{A}(A) \otimes \mathbb{H} \mathbb{A}(B) .
$$

2.3. Equivariant non-Archimedean periodic cyclic homology. An important motivation for the introduction of our homology theory is to study of group(oid) actions on affine varieties over a finite field. The orbit spaces of such actions are studied through crossed product algebras, which are noncommutative, and fit within the scope of application of our homology theory. In this realm, we wish to develop a non-Archimedean version of equivariant periodic cyclic homology $\mathrm{HP}^{G}$ in the sense of Christian Voigt $\sqrt[36]{ }$. We propose to investigate under what conditions, the following version of the Green-Julg Theorem holds: 
TheOREm. Let $G$ be a discrete group acting on a dagger algebra A. Then

$$
\mathrm{HP}_{\star}^{G}(V, A) \cong \mathrm{HA}_{\star}(G \rtimes A) .
$$

In more geometric situations, $A$ is the dagger completion of the coordinate ring of say a smooth, affine variety $X$. The idea would then be to relate these equivariant invariants to the more geometric, de Rham type invariants such as the rigid cohomology of the orbit space $X / G$.

2.4. Topological cyclic homology and final insights. An important development alongside 'classical' cyclic homology is topological Hochschild homology and its variants. These invariants are constructed by working over the sphere spectrum, instead of the derived category of $\mathbb{Z}$-modules. While topological versions of cyclic homology coincide with the classical versions in the Archimedean setting, their effect is most remarkable in the non-Archimedean setting. The pioneering work of Hesselholt and more recent work by Scholze-Nikolaus $([\mathbf{2 0}])$ and Petrov-Vologodsky $([\mathbf{2 9}])$ establishes connections between topological periodic cyclic homology and crystalline cohomology. However the methods used in the topological theories seem drastically different from our more concrete, analytical methods, which are better suited to the examples we find important. It is therefore very desirable to find connections between the two worlds. One possible approach could be the development of a bornological version of topological cyclic homology - a project I plan to take up in the future with Kobi Kremnitzer. Furthermore, I would also be interested in studying condensed mathematics developed by Scholze and Clausen, as a framework in which cyclic homology can be defined for topological algebras. 


\section{Bibliography}

[1] Gene Abrams, Pere Ara, and Mercedes Siles Molina, Leavitt path algebras, Lecture Notes in Mathematics, vol. 2191, Springer, London, 2017. doi: 10.1007/978-1-4471-7344-1|MR 3729290

[2] William W. Adams and Philippe Loustaunau, An introduction to Gröbner bases, Graduate Studies in Mathematics, vol. 3, Amer. Math. Soc., Providence, RI, 1994. doi: 10.1090/gsm/003 MR 1287608

[3] Alberto Arabia, Relèvements des algèbres lisses et de leurs morphismes, Comment. Math. Helv. 76 (2001), no. 4, 607-639, doi: 10.1007/s00014-001-8322-y. MR 1881700

[4] Michael Artin and Barry Mazur, Etale homotopy, Lecture Notes in Mathematics, vol. 100, Springer, 1969. MR 0245577

[5] Federico Bambozzi, On a generalization of affinoid varieties, Ph.D. Thesis, Università degli Studi di Padova, 2014. arXiv: 1401.5702

[6] Federico Bambozzi and Oren Ben-Bassat, Dagger geometry as Banach algebraic geometry, J. Number Theory 162 (2016), 391-462, doi: 10.1016/j.jnt.2015.10.023 MR 3448274

[7] Federico Bambozzi, Oren Ben-Bassat, and Kobi Kremnizer, Stein domains in Banach algebraic geometry, J. Funct. Anal. 274 (2018), no. 7, 1865-1927, doi: 10.1016/j.jfa.2018.01.003 MR 3762090

[8] Theo Bühler, Exact categories, Expo. Math. 28 (2010), no. 1, 1-69, doi: 10.1016/j.exmath.2009.04.004 MR 2606234

[9] Alain Connes, Entire cyclic cohomology of Banach algebras and characters of $\theta$-summable Fredholm modules, $K$-Theory 1 (1988), no. 6, 519-548, doi: 10.1007/BF00533785 MR 953915

[10] Guillermo Cortiñas, On the derived functor analogy in the Cuntz-Quillen framework for cyclic homology, Algebra Colloq. 5 (1998), no. 3, 305-328. MR 1679567

[11] Guillermo Cortiñas, Joachim Cuntz, Ralf Meyer, and Georg Tamme, Nonarchimedean bornologies, cyclic homology and rigid cohomology, Doc. Math. 23 (2018), 1197-1245, doi: 10.25537/dm.2018v23.1197-1245 MR 3874948

[12] Guillermo Cortiñas, Ralf Meyer, and Devarshi Mukherjee, Non-Archimedean analytic cyclic homology (2019), eprint. arXiv: 1912.09366

[13] Guillermo Cortiñas and Diego Montero, Algebraic bivariant K-theory and Leavitt path algebras, J. Noncommut. Geom., accepted. arXiv: 1806.09204

[14] Guillermo Cortiñas and Andreas Thom, Bivariant algebraic K-theory, J. Reine Angew. Math. 610 (2007), 71-123, doi: 10.1515/CRELLE.2007.068 MR 2359851

[15] Guillermo Cortiñas and Christian Valqui, Excision in bivariant periodic cyclic cohomology: a categorical approach, K-Theory $\mathbf{3 0}$ (2003), no. 2, 167-201, doi: 10.1023/B:KTHE.0000018383.93721.dd MR 2064238

[16] Joachim Cuntz and Daniel Quillen, Algebra extensions and nonsingularity, J. Amer. Math. Soc. 8 (1995), no. 2, 251-289, doi: 10.1090/S0894-0347-1995-1303029-0 MR 1303029

[17] _ Cyclic homology and nonsingularity, J. Amer. Math. Soc. 8 (1995), no. 2, 373-442, doi: 10.1090/S0894-0347-1995-1303030-7. MR 1303030

[18] Renée Elkik, Solutions d'équations à coefficients dans un anneau hensélien, Ann. Sci. École Norm. Sup. (4) 6 (1973), 553-603 (1974), available at http://www.numdam.org/item?id= ASENS_1973_4_6_4_553_0 MR 0345966

[19] Elmar Große-Klönne, De Rham cohomology of rigid spaces, Math. Z. 247 (2004), no. 2, 223-240, doi: 10.1007/s00209-003-0544-9 MR 2064051

[20] Lars Hesselholt and Peter Scholze, Arbeitsgemeinschaft: Topological Cyclic Homology, Oberwolfach Reports 15 (2019), no. 2, 805-940.

[21] Bernhard Keller, Chain complexes and stable categories, Manuscripta Math. 67 (1990), no. 4, 379-417, doi: 10.1007/BF02568439. MR 1052551 
[22] Masoud Khalkhali, Algebraic connections, universal bimodules and entire cyclic cohomology, Comm. Math. Phys. 161 (1994), no. 3, 433-446, available at https://projecteuclid.org/ euclid.cmp/1104270005 MR 1269386

[23] Joachim Lambek, Lectures on rings and modules, Blaisdell Publishing Co. Ginn and Co., Waltham, Mass.-Toronto, Ont.-London, 1966. With an appendix by Ian G. Connell. MR 0206032

[24] Ralf Meyer, Analytic cyclic cohomology, Ph.D. Thesis, Westf. Wilhelms-Universität Münster, 1999. arXiv: math.KT/9906205

[25] _ Excision in entire cyclic cohomology, J. Eur. Math. Soc. (JEMS) 3 (2001), no. 3 269-286, doi: 10.1007/s100970100035 MR 1848947

[26] _ Local and analytic cyclic homology, EMS Tracts in Mathematics, vol. 3, European Mathematical Society (EMS), Zürich, 2007. doi: 10.4171/039 MR 2337277

[27] Ralf Meyer and Devarshi Mukherjee, Dagger completions and bornological torsion-freeness, Q. J. Math. 70 (2019), no. 3, 1135-1156, doi: 10.1093/qmath/haz012 MR 4009486

[28] Paul Monsky and G. Washnitzer, Formal cohomology. I, Ann. of Math. (2) 88 (1968), 181-217, doi: 10.2307/1970571 MR 0248141

[29] Alexander Petrov and Vadim Vologodsky, On the periodic topological cyclic homology of DG categories in characteristic p (2019), eprint. arXiv: 1912.03246

[30] Fabienne Prosmans, Derived limits in quasi-abelian categories, Bull. Soc. Roy. Sci. Liège 68 (1999), no. 5-6, 335-401, available at http://popups.ulg.ac.be/0037-9565/index.php?id= 2128. MR 1743618

[31] Michael Puschnigg, Explicit product structures in cyclic homology theories, K-Theory 15 (1998), no. 4, 323-345, doi: 10.1023/A:1007748727044 MR 1656222

[32] Marius van der Put, The cohomology of Monsky and Washnitzer, Mém. Soc. Math. France (N.S.) 23 (1986), 4, 33-59, available at http://www.numdam.org/item?id=MSMF_1986_2_23_ _33_0 Introductions aux cohomologies p-adiques (Luminy, 1984). MR 865811

[33] Emily Riehl, Categories in context, Dover, 2017.

[34] Peter Schneider, Nonarchimedean functional analysis, Springer Monographs in Mathematics, Springer-Verlag, Berlin, 2002. doi: 10.1007/978-3-662-04728-6 MR 1869547

[35] Jean-Pierre Schneiders, Quasi-abelian categories and sheaves, Mém. Soc. Math. Fr. (N.S.) 76 (1999), vi+134, doi: 10.24033/msmf.389 MR 1779315

[36] Christian Voigt, Equivariant periodic cyclic homology, J. Inst. Math. Jussieu 6 (2007), no. 4, 689-763, doi: 10.1017/S1474748007000102 MR 2337312 